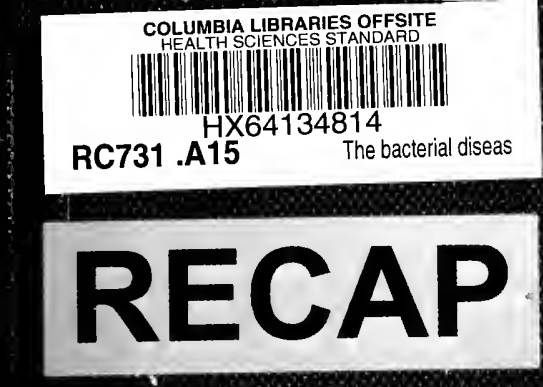




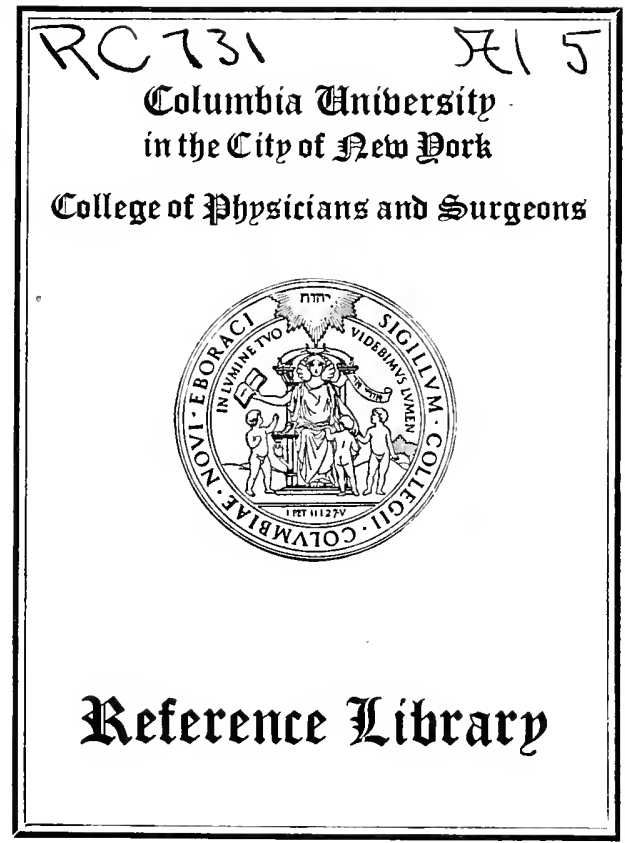







\section{THE BACTERIAL DISEASES OF RESPIRATION, AND VACCINES IN THEIR TREATMENT}

BY

R. W. ALLEN, M.D., B.S.(Lond.)

LATE EDITOR, "JOURNAL OF VACCINE THERAPY'; LATE CLINICAL PATHOLOGIST TO THE MOUNT VERNON HOSPITAL FOR DISEASES OF THE CHEST;

LATE PATHOLOGIST TO THE ROVAL EYE HOSPITAL; LATE GULL STUDENT OF PATHOLOGY, GUY'S HOSPITAL

PHILADELPHIA

P. BLAKISTON'S SON \& CO. 1012 WALNUT STREET 



\section{PREFACE}

M OST of the matter herein contained has already appeared as a series of articles in the numbers of the Journal of Vaccine Therapy from February, IgI2 to January, I9I3 inclusive. These have been revised and fresh matter included, the most important additions being in the sections devoted to pulmonary tuberculosis. Chapter XI is entirely new. My endeavour has been to treat my subjects in as practical a manner as possible; to lay before my readers the various considerations which should influence them in seeking help from specific treatment; and to point out those methods of application which a considerable practical experience has convinced me yield the best results.

To Dr. Ralph Vincent I am greatly indebted for taking the numerous excellent photographs and photo-micrographs which illustrate this book.

I28. Harley Street;

January, I9I3. 


\section{Digitized by the Internet Archive in 2010 with funding from Open Knowledge Commons}




\section{CONTENTS}

PAGE

INTRODUCTION

CHAPTER I.

The Bacteriology of the Respiratory Tract in Health

CHAPTER II.

The Respiratory Tract in Disease-Methods of InVESTigationTechnique of various Staining Methods . . . . . .

\section{CHAPTER III.}

Cultural Nethods for Investigating the BaCteriology of the Respiratory Tract-Description (ivith Plates) of the various PATHOGENIC BACTERIA WHICH MAY BE PRESENT

CHAPTER IV.

Results of Ofservations into the Bacteriology OF the various Diseased Conditions of the Respiratory TraCT-Nasal and Post-nasal Catarrh-Diseases of the Accessory SinusesEustachian Catarrh and Otitis Meda-Tracheitis and Laryngitis-Pulmonary Catarkh, Bronchitis and Asthma-WhoopingCough-Pulmonary Phthisis : . . . . .

CHAPTER V.

The Vaccine Therapy of Respiratory Disorders-General ConSIDERATIONS-CONTROL OF DOSAGE AND InTERVALS-SPECIAL CONSiderations-Preparation and Administration of the Vaccine

CHAP'TER VI.

Vaccines in the Treathent of Nasal and Post-nasal Catarrh, Tracheitis and Laryngitis, and Infections of the Accessory SPACES . . . . . . . . . 
CHAPTER VII.

Vaccines in the Treatment of Bronchitis and Asthua

CHAPTER VIII.

Vaccines in Pneunonia-Bacteriology-Prophylaxis-The PathoLogy and its Bearing on Vaccine Treatment: Technique and Results-Unresolved Pneumonia-Empyema and Lung Abscess -Broncho-PNEUMionia

\section{CHAPTER IX.}

VACCines in Whooping-Cough, Diphtheria, Pyorrhata alveolaris, Hay-Fever, Ozæna, Rhinoscleroma . . . . . . 12 I

\section{CHAPTER $\mathrm{X}$.}

The Mixed Infections of Pulaonary Tuberculosis and Vaccines in THEIR TREATMENT-IMPORT OF MIXED INFECTION-TECHNIQUE of Specific Therapy-Results-Prophylaxis

\section{CHAPTER XI.}

Infections ay the Tubercle Bacillus and the USE OF Specific Products in their Treathent-The Tubercle Bacillus AND its Toxins-The Defensive Mechanish of the Body-The TuberCulins and their Use in the Diagnosis of Pulaonary TuberCulosis: The Various "Tests"-Classification of Cases of Pulaionary Tuberculosis--Choice of Tuberculin-Control of DOSAGE AND INTERVALS-TREATMENT BY THE INDUCTION AND Control of Auto-inoculations-Conclusion. 


\section{THE BACTERIAL DISEASES OF RESPIRATION, AND VACCINES IN THEIR TREATMENT.}

\section{INTRODUCTION.}

IVHen first I began the study of this question just ten years ago, the one thing that struck me beyond all others was the utter inadequacy of the information obtainable from works in the English language, and, indeed, in any tongue, either bacteriological or dealing with general medicine, upon this very important subject. Despite the great impetus given to exact bacteriological research by the developments of vaccine therapeutics, especially within the past five years, the omission has not yet been repaired in any single book or collection of books to which I have had access. The true importance of any disease is to be estimated neither by its rarity nor by its mortality-rate, but rather by its frequency and its disabling power. Granting the truth of this statement, it follows that diseases of the respiratory tract are of supreme importance to the human race; in frequency they excel all others, in the production of impaired health of varying periods and to varying extents they are fruitful to an extreme degree. It has occurred to me that a brief but systematic description of the bacterial diseases of the respiratory tract would have a certain value and prove of interest to many. This I shall endeavour to carry out according to the following scheme.*

A. The Respiratory Tract in Health.

(i) Post-minortem results.

(ii) Results obtained during life; methods; carriers.

$B$. The Respiratory Tract in Disease.

(i) Methods of investigation.

* For the photographs and photo-micrographs I am entirely indebted to the kindness of Dr. Ralph Vincent. 
(a) Swabs from nose; precautions necessary in taking from turbinal bones, antra and sinuses.

(b) Secretion from nose; precautions necessary in collecting.

(c) Swabs from throat and Eustachian tubes; necessity of excluding or allowing for presence of pyorrhœa alveolaris, or tonsillitis (follicular or otherwise).

(d) Sputum ; precautions necessary in collecting.

(e) Lung puncture.

$(f)$ Examination of the specimen.

(I) Chemical determination of source; salol test ; albumen test.

(2) Staining methods for cells and bacteria.

(3) Cultural methods.

(ii) Description of the bacteria which may be present :

Tubercle bacillus, *lepra bacillus, *Streptothrix actinomycosis, *Spirochata pallida, *Staphylococcus albus, aureus, citvens, candicans, Streptococcus group, pneumococcus, B. influenza, B. Bordet-Gengou of whooping-cough, *bacillus of Koch-Weeks, B. diphtheria, *B. Hoffmanii, ${ }^{*} B$. xerosis, B. septus, M. catarrhalis group, M. paratetragenus, $M$. tetragenus, bacillus of Friedlander group, $B$. protens group, B. pyocyanens, B. coli, B. typhosus, *various organisms associated with pyorrhœa alveolaris and tonsillitis, such as vibrios, streptothrices, micrococci and fusiform bacilli, $\dagger$ B. ozana, $\dagger$ B. of rhinoscleroma.

* To these bacteria short reference only will be made.

+ For description of these bacteria see Chapter IX.

(iii) Results of observations.

(I) In nasal and post-nasal catarrh.

(2) In diseases of the accessory sinuses.

(3) In Eustachian catarrh and otitis media.

(4) In tracheitis and laryngitis.

(5) In pulmonary catarrh, bronchitis and asthma.

(6) In whooping-cough.

(7) In pulmonary phthisis.

(iv) Vaccine therapy of respiratory disorders.

(a) General considerations.

(b) Special considerations.

(I) Nasal and post-nasal catarrh, tracheitis and laryngitis, and infections of the accessory spaces.

(2) Bronchitis and asthma. 
(3) Pneumonia.

(4) Whooping-cough.

(5) Diphtheria.

(6) Pyorrhœa alveolaris.

(7) Hay fever.

(8) Ozæna or atrophic rhinitis.

(9) Rhinoscleroma.

(Io) Pulmonary tuberculosis.

(a) Mixed infections: their import and treatment.

(b) Infection by the tubercle bacillus and the use of specific products in treatment. 


\section{CHAPTER I.}

\section{A. THE BACTERIOLOGY OF THE RESPIRATORY TRACT IN HEALTH.}

It is only those who have devoted years to research in one of the "exact sciences," such as physics or physical chemistry, who can adequately realise the supreme importance to any investigation of the performance of sufficient "control" observations. It is owing to the peculiar difficulty in securing subjects which can in any way be regarded as absolutely normal or healthy individuals, and as suitable, therefore, to act as "controls," that medicine has failed in the past, and, perforce, will fail in the future, to take a place among the exact sciences. The multiplication of careful control observations upon individuals as healthy as can be obtained in regard to the parts under study serves to a considerable degree to eliminate erroneous conclusions.

The artificial conditions of modern life have rendered it peculiarly difficult to secure for investigation an adequate number of individuals any part of whose respiratory tracts could be regarded as strictly healthy, especially from the bacteriological standpoint. It may be recalled that some people appear constantly to harbour the Micrococcus catarrhalis in their nasal passages, yet only very occasionally suffer from acute nasal catarrh, and then for strictly limited periods only, whilst from the throats of others the pneumococcus or Bacillus diphtherice can be constantly recovered, yet never have they suffered from pneumonia on the one hand or diphtheria on the other. The study of these so-called "carriers" would tend to the conclusion that these, therefore, could not be pathogenic bacteria, yet the contrary we know to be the case.

It is perhaps fortunate that the results of vaccine treatment in diseases of the respiratory tract enable us to confirm deductions made from observations inherently fallacious and difficult to control.

In any bacteriological research it is above all essential that two conditions be fulfilled: (I) that the material be obtained from the 
desired situation without chance of contamination in the process; (2) that it be speedily prepared in film form for direct examination; this done, that it be immediately transferred to suitable media for cultural observation. By suitable media is meant such as each and every one of the bacteria present may find adapted to their growth.

Through failure to comply in one or other direction with these prime essentials practically all investigators upon the respiratory tract, with the exception of some who have worked within the last five or six years, have rendered their observations so untrustworthy as to be almost valueless. As the procedures adapted to the study of pathological conditions are a fortiori suitable in normal or healthy states, their consideration is deferred to the next section upon the bacteriology of the respiratory tract in disease.

Inasmuch as many of the domestic animals, such as cattle, horses, cats, dogs, and rabbits are susceptible to respiratory disorders like those of man, the comparative study of the respiratory systems of these animals in health and disease would prove illuminating; unfortunately this has not been done, and such information as we have is therefore incomplete. Observations upon the dead human subject are also liable to prove very misleading owing to "agonal spasms" and other causes, except in the instance of those who have died a violent death ; investigation upon these latter is wholly lacking.

The recent observations upon animals by Cobbett (Proc. Roy. Soc. Med., July, I9II, p. 205) have, however, brought out clearly one important fact. He there states that he has never failed to find bacteria in the lungs of the various animals that he examined-these were killed instantaneously and examined at once so that agonal and post-mortem infection can be absolutely excluded. Among the germs he commonly found in the lungs of rabbits and guinea-pigs were moulds, streptothrices, spore-bearing bacilli and cocci-in fact, just the ordinary micro-organisms of the air. Spraying experiments by Hurtl and Hermann and by Bastel and Neumann have been confirmed by Cobbett, and show that air-borne bacteria freely enter the air-passages and get carried to the lung; the bronchial mucous membrane, like the intestinal, must, therefore, for practical purposes be regarded as part of the surface of the body.

Nor is this the only route whereby bacteria reach the lungs of the normal and so a fortiori of the unhealthy individual. While it is probably true that the various lymph-glands, and especially the bronchial, cervical and abdominal ones, are very efficient guardians of the general circulatory system against bacterial infection, it yet certainly happens that occasionally bacteria penetrate the defence and find ingress into the blood-stream. The two organs in particular which from their 
structure are peculiarly adapted for the filtration of these chance invaders are the lungs and spleen; it thus happens that a certain number of bacteria reach the lung via the circulation.

That the air, especially of cities, is laden with bacteria is common knowledge; the proportion of pathogenic to non-pathogenic organisms is, however, small. Experiments upon purely nose-breathers has shown that the filtration of the air in the nasal passages is efficient to an extraordinary degree; the mouth-breather loses this protective action, and is consequently much the more prone to respiratory disorders. It is of obvious interest to know in what part of the nasal passages this daily host of bacteria is stopped and what is their fate. One fact upon which all investigators are agreed is that the vestibule of the nose is swarming with bacteria; another is that in health the accessory airspaces and the upper meatus of the nose are approximately sterile. The precise degree of bacterial infection commonly existent below the level of the middle turbinal has not been accurately determined owing to faulty methods of observation. The most satisfactory published investigations are those of Lewis and Logan Turner (Edin. Med. Journ., I905, vol. ii, p. 393). It is to be regretted that their cultural methods were not beyond criticism, the failure to make an invariable rule of employing blood-agar as a medium being a very important omission. By means of smears and cultures they examined 26 specimens from I6 persons; only 3 of these proved to be sterile.

I3 specimens from 7 persons showed only one variety of bacterium.

$\begin{array}{lllllll}9 & , & & & & & \text { two varieties } \\ \text { I } & , & , & \text { I } & , & , & \text {, }\end{array}$

Staphylococcus albus was present in 13 specimens.

Streptococcus

Pneumococcus

Hoffmann's bacillus

Staphylococcus aureus

Bacillus mesentericus

Spirillum

Bacillus of Friedlander

Proteus vulgaris

$\begin{array}{lll}, & 6 & , \\ , & 4 & , \\ ", & 2 & , \\ , & 2 & , \\ , & 2 & , \\ , & 2 & , \\ , & \text { I specimen }\end{array}$

The pathogenicity of the organisms was determined upon rabbits and guinea-pigs in eleven instances-an investigation, in my opinion, entirely lacking value and only likely to prove misleading. Nine of them appeared to be non-pathogenic, two were pathogenic, viz. a streptococcus and a Staphylococcus aureus. Their conclusion was that the organisms of the healthy nose belong to the same varieties as those found in abnormal conditions, but differ in that they-

(I) Are present in much fewer numbers. 
(2) Afford purer cultures.

(3) Possess less vigorous power of growth.

(4) Are of low pathogenicity.

These observations are in practical accord with my own, and it would appear that by far the greater proportion of the bacteria which enter the healthy nose are deposited in the vestibule and the inferior meatus.

Of their precise fate we know little. Many are removed by the use of the handkerchief ; some certainly are phagocytosed by the epithelial cells, which possess this power to a very high degree; whether any undergo lysis by the aid of the mucus is unknown. That nasal mucus is absolutely devoid of bactericidal power I showed some years ago; that it may possess a lytic action is possible, but appears not to be the case from a number of experiments which I have made to elucidate this point.

Any attempt at discussion as to what is the bacteriology of the healthy mouth would be of purely academic interest, in as much as the perfectly healthy mouth is for all practical purposes non-existent. Somewhere or other in every mouth or pharynx careful search will reveal a focus of disease: it may be a carious tooth, inflamed dental papillæ, a condition of pyorrhœea alveolaris, confined perhaps to the gum round a wisdom tooth or behind the upper incisors, follicular tonsillitis, an infection of the supra-tonsillar fossa difficult of detection, a granular pharyngitis or some other condition giving rise to little or no discomfort, but none the less surely there. A perusal of the voluminous writings upon the bacteriology of these conditions would lead to but one conclusion: that if one or the other of these conditions be normally present in the mouth or pharynx, then so varied is the bacterial content already of the oral cavity that it must surely be a hopeless task to endeavour to elucidate the bacteriology of any other focus of disease in or near the mouth or pharynx. In actual practice the diffculty is by no means as great as it would appear, provided that due recognition be paid to the possible fallacies thereby introduced, and measures be taken for their exclusion; how this can be done will appear presently. Of the organisms which have been found in more or less normal oral cavities the following is a very imperfect list: Staphylococcus albus and aureus, streptococcus, Micrococcus catarrhalis, various small micrococci, both staining and failing to stain by Gram's method, vibrios, spirilla, spirochætæ, fusiform bacillus, $B$. necrodentalis, $B$. buccalis maximus, Cladothrix buccalis, $B$. proteus, $B$. mesentericus, $B$. subtilis, B. fluorescens, sarcinæ, yeasts, and moulds. 


\section{CHAPTER II.}

\section{B. THE RESPIRATORY TRACT IN DISEASE.}

\section{(I) Methods of Investigation.}

These are to be pursued upon material obtained either by oneself or by one more highly trained in the technique of catheterising the various accessory air-spaces, or else upon material supplied by the patient. By each and every one of these special precautions must be observed in order that the bacteriological findings may not be unduly obscured. Some of the more important points may here be mentioned.

(a) In the securing of "swabs" from the turbinal bones, septum, antrum, and accessory air-cells and Eustachian tubes the nasal route must perforce be adopted. To the fact that the vibrissæ, vestibule and inferior meatus are swarming with the bacteria caught up from the airstream attention has been already drawn. It is therefore obvious (I) that these bacteria should be removed as far as possible; (2) that contact with these parts by the swab or loop must be carefully avoided. The former object is attained, for all practical purposes, by washing the hands well with hot water and ethereal soap, then by means of the finger-tips, moistened with soap and hot sterile water, the orifice of the nose is thoroughly cleansed; a pledget of sterile wool moistened with absolute alcohol is employed to swab out the orifice. A sterilised nasal speculum is next inserted, and through it the swab or platinum loop is carefully passed and secretion taken up from the desired spot. Adequate illumination and considerable technical skill are requisite if the materies is to be collected from the Eustachian tube, antrum or accessory sinuses. Particular care must be directed to the withdrawal of the swab or loop as contamination is then particularly liable to occur. The methods of examination of the specimen by direct observation of suitably stained films and by cultural examination will be detailed later. Attention may, however, be drawn to this fact that inasmuch as specimens of nasal mucus frequently contain but few organisms, so few as to be difficult of detection in film preparations, a sufficiency of material must be collected not only for the insemination of plates, but also for the preparation of quite thick smears. 
(b) If the specimen of mucus is to be furnished by the patient, instruction must be given in this method of cleansing the nasal orifice, and two sterilised, wide-necked bottles be supplied: two bottles are requisite for this reason, that especially in cases of antral or sinus infection the bacteriology of the two sides may by no means be the same; the necessity of securing specimens from both sides is therefore obvious.

The patient receives the following instructions: Immediately on waking the nose is cleansed as indicated above, the stopper removed from one bottle, which is then held against one orifice, the other being closed by means of a finger-tip; a forcible nasal effort then expels some of the secretion directly into the bottle, which is at once stoppered. This operation is repeated with the other side, and the specimens forwarded for examination without delay.

(c) and $(d)$ Considerable as is the care necessary for the collection of nasal specimens, it is relatively quite inconsiderable to that requisite for the collection of secretion from the naso-pharynx, pharynx, and lower respiratory tract. Of all the cavities of the body the mouth and pharynx bacteriologically are incomparably the foulest; 90 per cent., at least, of all residents in the United Kingdom carry in their mouths foci of bacterial infection-undetected pyorrhœa alveolaris ravages their gums, poisons them locally and generally, impairs the digestion, the appetite and general health, produces chronic invalids, gives rise to obscure pains and pyrexias, complicates respiratory phthisis, and, let it well be borne in mind, of itself produces a premature end in by no means an inconsiderable number of cases. Follicular tonsillitis also frequently escapes the eye, not only of the patient, but likewise of the medical man: cursory examination will by no means suffice for its detection, especially when the follicles infected by those immediately posterior to the anterior pillar of the fauces or those of the supratonsillar fossa; careful pressure upon and around the anterior pillar will frequently reveal an unsuspected focus of infection. Into the bacteriology of these conditions, which is so characteristic as to reveal their presence when naked-eye observation has defaulted, I shall enter fully later, but let it here be mentioned that several of the commoner bacterial agents of these conditions are precisely those responsible for catarrhs of the respiratory tract, colds, asthma, bronchitis, pneumonia, and the mixed infections of phthisis; others which are not responsible for these conditions also are productive of extra-cellular toxins so potent that one minim of a sterilised broth culture injected subcutaneously may suffice to produce vomiting and rigors.

When, therefore, a patient presents himself for treatment of a respiratory disorder, and pharyngeal, laryngeal, or bronchial specimens 
are contemplated, it behoves us first and foremost carefully to scrutinise the mouth and pharynx; to examine the gums, especially behind the upper incisors and around the back molars and wisdom teeth, for the presence of pyorrhoea alveolaris, the tonsils for inflamed and infected crypts and follicles-and here let me urge upon your notice, crowned teeth or bridges practically invariably indicate an accompanying pyorrhøa, for the recession of the gum has given ample room for the fixation of the denture. Such supreme importance do I now attach to these conditions in respiratory $y_{\infty}$ disorders, that should I detect their presence and the patient refuse to have them properly treated, then I firmly and finally refuse to undertake the treatment of the respiratory condition. To allow oneself to be so handicapped at the outset is foolish beyond measure.

The bacterial content of the mouth incidental to food residues also needs elimination. All fallacies introduced by the above causes may be satisfactorily eliminated by the following procedure, which merely needs the more careful performance should pyorrhæa or tonsilitis have been detected. A jug of well-boiled water is provided, and a new, moderately hard tooth-brush taken and dipped for a few seconds twice or thrice into the boiling water, which is then cooled to a comfortable temperature. The teeth are now thoroughly brushed with the new brush and sterile water, no powder or antiseptics being employed. The mouth is well rinsed out and the throat gargled with the water, of which two or three mouthfuls then are swallowed. Swabs are now taken from the naso-pharynx, pharynx, tonsils or larynx, and employed for direct and cultural examinations in the manner presently to be related. Should the area of infection be too low in the respiratory tract for us to reach, the patient is instructed to carry out the above procedures immediately on waking, then give one, or at the most two, coughs, and expectorate directly into the wide-necked sterile bottle with which he has been furnished. If accurate bacteriological investigation be desired, too much stress can hardly be laid upon the due observance of these preliminary precautions.

(e) Lung puncture.-Occasionally in cases of suspected lung infection no sputum can be voided for examination-a dilemma in which I myself have been placed on at least three occasions. Recourse must then be had to puncture of the lung, a procedure altogether void of danger if due precautions be observed, but not devoid of pain. Briefly, the method is as follows: Careful percussion and auscultation having revealed a suspected area the skin is sterilised, best with strong tincture of iodine, and may then be anæsthetised with ethyl chloride spray. A 5-Io c.c. all-glass syringe with a strong wide needle is sterilised by boiling and $\mathrm{I}$ or 2 c.c. of sterile peptone broth taken into it. The 
patient is then instructed to expire and cease respiratory movement; in this way tearing of lung tissue and resultant pain is minimised. The needle is plunged boldly into the selected area, $0^{\circ} 5^{-1}{ }^{\circ} \mathrm{o}$ c.c. of the broth expelled, and gentle aspiration applied; if necessary the needle may be withdrawn a little or inserted further and the procedure repeated. The specimen so obtained is to be examined by direct and cultural observation; the results are very satisfactory as risk of contamination is minimal; in this way I have secured cultures of $B$. influenza, pneumococcus, and $B$. typhosus directly from the lung.

(f) Examination of the materies morbi: (I) Chemical determination of source.-The mere bacteriological examination, direct and cultural, will not suffice for all cases. To enter into a full discussion of the constituents of the sputum and nasal secretion would be beyond the scope of this paper; attention may, however, be briefly directed to the following few points. Among the more important constituents of the sputum are: water, salts, cells, mucin, serum albumen (coagulated and uncoagulated), serum globulin, ferments, excreted medicaments, and bacteria. These vary within very wide limits under different conditions.

To the chemical constituents of the sputum little attention has been paid in practical medicine; the following recent observations, however, are of considerable interest, and may prove of great service in the diagnosis of obscure lung conditions.

Falk and Tedesco (Wien.med. Woch., I gog, xxii, 954) have noted the fact that salicylic acid present in the blood appears in inflammatory exudates in the lung, but is not excreted by the bronchial mucosa; the application of the following test will therefore assist in differentiating disease processes limited to the bronchial mucosa from those which have extended to the lung.

Thirty grains of sodium salicylate are given by the mouth and the sputum collected during the ensuing twelve to eighteen hours. It is slightly acidified with acetic acid and shaken thoroughly with five times its volume of absolute alcohol. The mucus and albumen are precipitated in coarse flocculi and removed by filtration. The clear filtrate, which contains all the salicylate acid, if any be present, is rendered slightly alkaline, and evaporated to dryness over a water bath; the residue is dissolved in water, slightly acidified, and sugar of lead added till no more precipitate forms: this latter is removed by filtration and washed with water, the washings being added to the acid filtrate, which is then extracted with ether. The ethereal extract is evaporated to dryness, the residue dissolved in Io c.c. of water, and I c.c. of ro per cent. aqueous solution of ferric chloride is added. The formation of a violet colour indicates the presence of salicylic acid. Patients suffering from catarrhal bronchitis, bronchitis, emphy- 
sema, bronchial asthma, purulent bronchitis, and bronchiectasis all gave negative results, whereas cases of lobar pneumonia reacted strongly.

The reaction gradually lessens until the crisis is reached, when it rapidly disappears, and varies in intensity roughly with the extent of lung affected. In phthisis the results varied; a positive result was always obtained, but the intensity was not proportional to the clinical severity or the extent of the process. Generally, however, acute cases gave a stronger reaction than chronic. Falk and Tedesco hold that a repeatedly negative result is strong evidence that the process is limited to the bronchial mucosa, and is therefore exclusive of pneumonia or pulmonary phthisis.

Lesieur and Prirez (Paris Médical, IgII, vol. iv, p. 29) have investigated the sputum voided in various conditions for albumen content: 5 c.c. of sputum are taken; to this 20 c.c. of normal salt solution and five or six drops of acetic acid are added. The mixture is well shaken and filtered; the filtrate is then tested for albumen by boiling or with nitric acid. If the reaction by boiling be doubtful the addition of a further 20 c.c. of salt solution and fresh boiling will sometimes give a positive result. Lesieur and Prirez found that of cases without physical signs which subsequently proved to be tuberculous 75 per cent. gave a positive reaction; that all cases with tubercle bacilli in the sputum were positive; that in miliary tuberculosis and pleurisy the result was not constant ; that cases of acute lobar pneumonia reacted, and that when the reaction persisted into convalescence a new focus or a complication was indicated. Acute broncho-pneumonia and acute pulmonary œdema were also positive. On the other hand, in acute bronchitis it was usually negative, in chronic bronchitis and in emphysema always so. In cardio-renal cases a positive reaction was often seen.

In 840 observations by various authors the reaction was given in Ioo per cent. of cases where tubercle bacilli were present, in 82 per cent. of cases which were probably tuberculous and in 37 per cent. of non-tuberculous cases. As with the salicylic acid test, it would thus seem that a positive reaction indicates a pulmonary origin of the exudate and excludes a bronchial source, and that the more marked and more constant the result the greater the likelihood of phthisis or pneumonia.

(2) Staining methods for cells and their derivatives. - The most complete account of the constituents of the sputum other than bacterial and how these vary in health and disease is to be found in Etude Histochimique et Cytologique des Crachats, by Israëls de Jong, from which I have taken many of the following particulars. The staining method which de Jong found most generally useful is as follows: A thin film of the secretion is prepared and allowed to dry without the application of 
heat; it is then fixed for two or three seconds in I per cent. aqueous solution of chromic acid, washed well under the tap, stained for three minutes in undiluted polychrome methylene-blue of Unna, differentiated with 90 per cent. alcohol, washed well with water, dried by the application of gentle heat and examined by the aid of artificial light. Owing to the metachromatic properties of this stain, red corpuscles, mucin, epithelial cells, leucocytes and their granules are coloured differently. The red cells appear green, mucin reddish, the nuclei of the epithelial cells characteristically a deep violet, almost black, their cytoplasm a light violet to lilac, the nuclei of the leucocytes a deep violet to black, their cytoplasm a light violet, the granules of mastcells reddish; those of the eosinophiles are unstained, yet clearly visible on account of their refractility and double contour.

Unna's polychrome methylene-blue is a by no means easy stain to manage, and requires considerable practice in order to obtain satisfactory results. As an alternative, still better as a supplementary method, one of the various modifications of Romanowsky's stain, preferably Leishman's, may be employed. Here let me just mention that if one makes up the liquid stain for himself the following points must be observed: (I) Use only Grubler's stain and the purest methyl alcohol; (2) dissolve $0.4 \mathrm{grm}$. of the former in $200 \mathrm{c.c}$. of the latter, place in a well-corked flask, shake daily for a fortnight, and filter thoroughly when transferring to the stain bottle; at least a fortnight's maturation of the solution is requisite.

The technique for Leishman's stain is as follows: Films should be made upon well-cleaned slides, spread thinly and allowed to dry in the air without the application of heat. The slide is covered with the stain, which is allowed to remain for three minutes in hot weather, for five in cold; the preparation is then flooded with distilled water, which is well mixed with the stain by means of a pipette. The mixture is allowed to remain for fifteen minutes, then poured away and the slide plunged into plenty of distilled water; in this the preparation is agitated until no more stain comes away; it is then washed with fresh distilled water, lightly blotted between filterpaper and dried by warming gently. Examination is best made without the use of a cover-slip. Bacteria are stained blue, cell nuclei dark blue, cytoplasm lilac or pink according to the cell, basophile granules violet, acidophile granules red, red blood-corpuscles red, mucin a dirty blue.

The cells of the pharyngeal mucosa (Plate I, fig. I) are very large cells, irregularly polygonal in shape, with a large oval nucleus placed either centrally or near the periphery. WVith Unna's stain the nucleus of the undegenerated cell takes on a deep violet colour, the cytoplasm a feeble blue-violet. With Leishman's stain the nucleus 
is deep blue, the protoplasm reddish-violet. These cells are frequently seen crowded with bacteria, especially when beginning to degenerate (as in Fig. I). The degenerative process proceeds rapidly, the cell then having a greater affinity for acid than for basic stains, so that the cytoplasm may appear a bright red with Leishman's stain.

The cells of the bronchial mucosa (Plate I, figs. II $a$ and II $b$ ).The bronchial mucosa consists of several layers of stratified cylindrical epithelium with a superficial ciliated layer. Normal bronchial cells are rare in sputum except in cases of severe early trancheitis or in acute asthma; in these instances violent fits of coughing may lead to the tearing off of small portions of the mucosa ; even here a certain amount of degeneration has as a rule set in. The ciliated superficial layer consists of a cylindrical cell with large oval nucleus. The outer end of the cell is flat and covered with ciliæ; the other end is drawn out, sometimes bifid, and insinuates itself beneath the neighbouring cell or cells (Fig. II $a$ ). The deeper cells are polyhedral or shaped like a truncated cone with large nuclei.

By Unna's stain the nucleus of the normal cells stains violet and uniformly, the cytoplasm bluish. The normal cell, as said before, is very rarely seen ; usually degeneration has already set in. The nucleus then appears reticulated, swollen, and occupying perhaps two thirds of the cell; later it may be drawn out into a long network protruding from the cell (Fig. II $b$ ); still later the cytoplasm may entirely disappear and the nuclear reticulum be drawn out into long strands, which have often been mistaken for fibrin, and may include polynuclear cells in their network-an appearance often seen in acute and chronic bronchitis. The cytoplasm of the degenerating cell with Unna's stain appears of a lilac colour, the nucleus, unlike that of most other cells, stains reddish with a slight violet tinge like mucin.

Leishman's stain is but ill adapted for showing the bronchial cells in their various stages of degeneration; with it the nuclear reticulum appears lilac to blue, according to the amount of washing to which the preparation is subjected.

The cells of the pulmonary alveoli (Plate I, figs. III and IV).These, unlike the bronchial cells, are frequently to be seen in the sputum in their more or less normal condition, as well as in their degenerative forms.

The normal alveolar epithelium consists of a thick layer of large plaques without nuclei bound together by a kind of cement; between these lamellar cells are small dark protoplasmic areas with well-defined nuclei. The layer of unnucleated cells covers the capillary network, whilst the nucleated protoplasm occupies the mesh of the blood-vessels. 


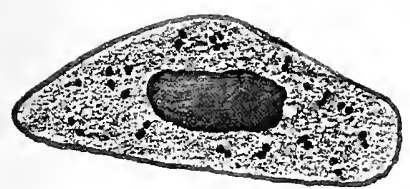

Feg $z$

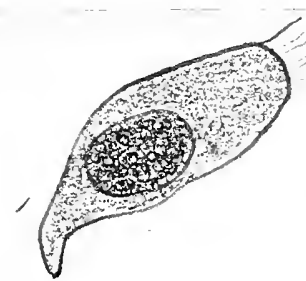

Fiz II

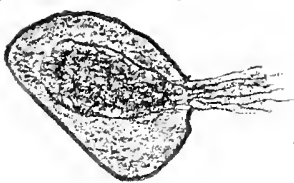

Fig
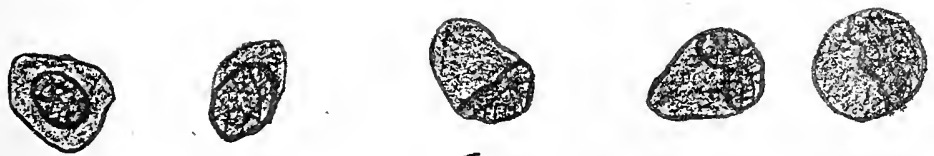

Fig III
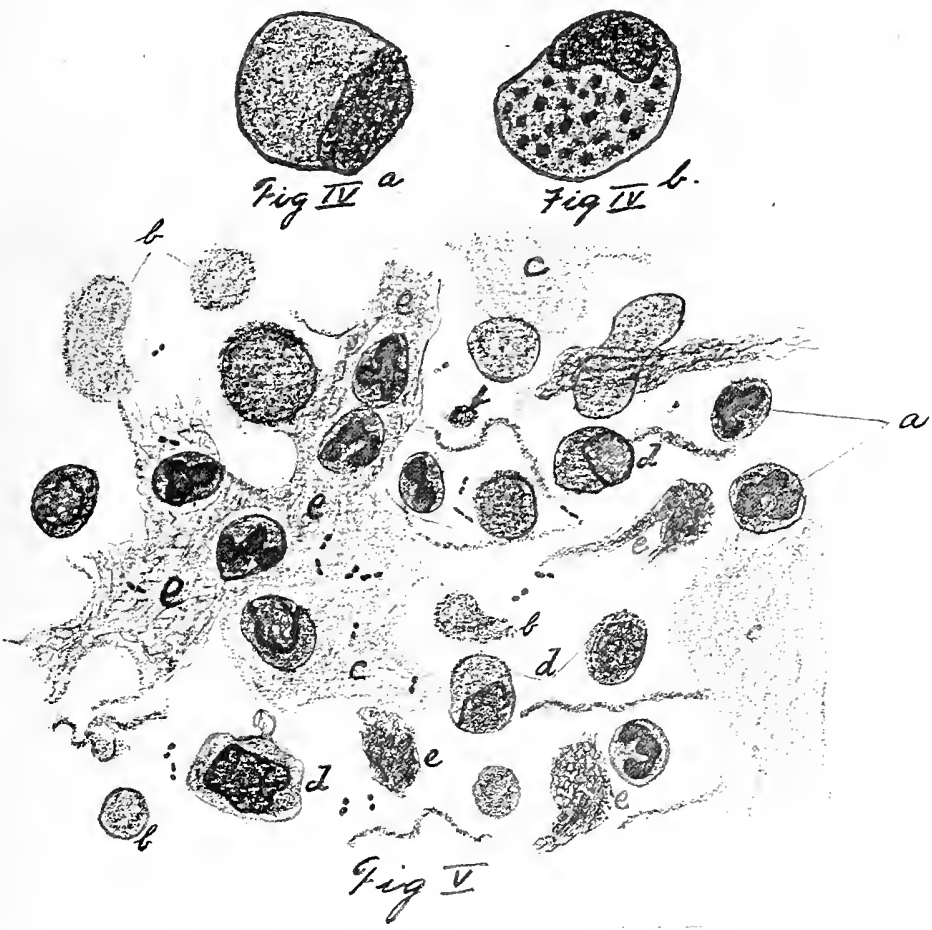

Diagrammatic representations of constituents of sputum (after Israels de Jong). Fig. I. Pharyngeal cell with bacteria. Fig. II $a$. Cell of superficial layer of bronchial mucosa. Fig. II $b$. Bronchial cell undergoing degeneration with extrusion of nucleus. Fig. III. Small pulmonary cells. Fig. IV a. Macrophagic pulmonary cell. Fig. IV b. Ditto with pigmentgranules. Fig. V. Sputum of pneumonia at about eighth day : (a) Polynuclears; (b) Seroalbuminous exudate; (c) Hyaline mucus: (d) Pulmonary alveolar cells; (e) Mucinous reticulum derived from cell nuclei, and containing in its mesh polynuclear cells and bacteria. 

The precise origin of the unnucleated plaques is unknown, but they are probably derived from the small nucleated cells.

In the sputum these cells may appear in the following forms: (a) As the above small nucleated cell (Figs. III and $\mathrm{V} d) ;(b)$ as a large macrophagic cell often laden with pigment (Figs. IV $a$ and IV $b$ ); (c) as a degenerated reticulum. The small nucleated alveolar cell somewhat resembles the mononuclear cells of the blood, but differs from them in certain particulars. The nucleus is round or oval, occupying the centre of the cell and filling about twothirds of it. The cell itself is small, round, or slightly oval, the cytoplasm apparently granular. With Unna's blue the nucleus stains a dark violet, the cytoplasm a much lighter violet, and showing innumerable tiny basic granules; this cell is very common in the sputum of pneumonia, broncho-pneumonia, pulmonary congestion, softening phthisis, and capillary bronchitis; it is rare in acute bronchitis and in emphysema with bronchitis. If they make their appearance during the course of a bronchitis they indicate capillary extension, pulmonary congestion, or an area of pneumonia; these cells are distinguished from the mononuclears of the blood, which probably appear but very rarely in pulmonary exudates, by their nucleus being more oval, more compact, and staining too deeply, by the protoplasm being more abundant, and with greater affinity for stains.

The endothelial macrophages (Figs. IV $a$ and IV $b$ ) are large cells with an excentric nucleus; sometimes they are bi- or even tri-nucleated; sometimes the cytoplasm is abundant, vacuolated, and stains feebly except at the periphery; sometimes it is quite scanty and stains deeply. Between this cell and the small alveolar cell there are all gradations, and the former is probably only a specialised form or derivative of the latter.

The macrophages are often laden with particles of pigment, derived either from the air, when they are deep brown or black, or from the blood-pigment, when they are golden (Fig. IVb). They are found in such conditions as resolving pneumonia and broncho-pneumonia, in bronchitis with pulmonary congestion, and in pulmonary congestion due to granular kidney.

As with the bronchial cell, so with the pulmonary in an advanced stage of degeneration, all that may remain of the cell is a reticulum derived from the nucleus, and having the staining reactions of mucin. With Unna's blue they stain red and more deeply than the bronchial reticula, from which they also differ in being more compact, less drawn out and less fine, and in frequently containing bacteria and leucocytes in their mesh (Fig. Ve). With stains such as Leishman's this reticulum preserves its basis affinity much more strongly than does that derived from the bronchial cell; sometimes the reticulum appears to 
preserve the outline of the cell; sometimes those derived from several cells appear to unite to form a larger network.

Blood-cells in the sputum.-For the study of these elements no stain is so well adapted as that of Leishman; their appearances are too well known to need description. It will suffice to mention ( $\mathrm{I}$ ) that red cells are found in any form of acute pulmonary congestion, and that their appearance in the sputum of bronchitis is indicative of alveolar extension; (2) that degenerative changes occur, the nuclei of polymorphonuclears undergoing extrusion, lying as a long thread attached to the cell or by the side of it, and staining the reddish colour characteristic of mucin, whilst the granules, especially of the coarsely granular eosinophiles, are frequently shed from the cells as if by a violent explosion. This is especially well seen in sputum from a case of acute asthma, in which disease only, tuberculosis excepted, do coarsely granular eosinophiles appear in considerable numbers in the sputum.

The mucin of the sputum (Plate I, fig. Vc).-So much attention has been drawn to the degeneration of the nuclei of the bronchial and pulmonary cells as a source of mucin that the question may well be asked, Is this the source of all the mucin of the sputum? The answer is, Certainly not; the great bulk of sputum is composed of mucus secreted by the special mucous glands, the product apparently of their nuclear activity, but differing from that previously described by its hyaline non-reticular structure (Fig. $\mathrm{Vc}$ ). These glands are most numerous in the trachea, less so in the bronchi; as the bronchi approach the lung the mucous glands become ferver and fewer until they cease to exist in the smaller bronchioles.

It may appear somewhat superfluous further to labour the point that much of what has been described in the past and is regarded at the present as fibrin in sputum is not fibrin, but mucin, but the point is a very important one, for fibrin is the coagulated form of the seroalbuminous exudate from lung-tissue proper, the significance of which has already been referred to under the salol and albumen tests.

The sero-albuminous constituents of sputum (Plate I, fig. Vb).-As has been already stated, mucin stains reddish to reddish-violet with Unna's blue, violet to blue with Leishman's stain, and very feebly blue with Weigert's stain; fibrin, on the other hand, stains blue-grey to blue-green with Unna's blue, red with Leishman's stain, and deep blue by Weigert's method. The fibrinous reticulum is composed of straight fibrils crossing each other in every direction, while the mucous reticulum is wavy, and composed of fibrils which anastomose in various curves.

If the sputum of such conditions as acute pulmonary cedema, 
phthisis, or early pneumonia be stained, by the above methods numerous rounded masses which often seem to coalesce are seen stained violetblue or blue-grey. These droplets vary in size and shape (Fig. Vb) : the smallest have about thrice the diameter of a red blood-cell and are round; the larger are oval; larger still theyare irregular and lobulated as if by the coalescence of several droplets-in acute pulmonary œdema practically the whole of the exudate consist of this body; in pneumonia they appear during the stage of hepatisation and disappear with the redux crepitation; they may constitute the whole groundwork of the preparation as if the droplets had all run together.

Inasmuch as precisely similar effects are obtained by suitably stained serum, pleural exudate, ascitic fluid or albuminous urine, there is little doubt that this substance is derived from the blood, and like the albuminoid constituents of the blood, possesses a somewhat complex constitution.

We now pass on to consideration of the staining methods suitable for demonstrating the bacterial flora of the respiratory passages in smear preparations and in culture films. The payment of attention to the following points will be found distinctly advantageous:

(I) Smears and films alike should be made, not upon cover-slips, but upon new, well-cleaned slides; the gain in ease of manipulation is considerable.

(2) Due heed should be paid to the selection of the specimen; it is little use selecting a piece of laryngeal or tracheal mucus when the desire is to ascertain the pulmonary conditions as regards bacterial contents. Sometimes it is necessary to prepare multiple smears, for even to the naked eye the specimen supplied may not present a homogeneous appearance. Thin films have a distinct advantage over thick ones except when we are dealing with nasal mucus, and it is not desirable to cover more than, say, the middle third of the slide with material.

(3) Stains which are liable to sedimentation should be carefully filtered immediately prior to use.

(4) A good lens is a prime necessity and a movable stage a decided advantage; it should be made an invariable rule to use either natural or artificial illumination, not sometimes one, sometimes the other.

(5) The gravity of an infection is not necessarily proportional to the number of bacteria present in a smear, nor in mixed infections is the relative import of the several invaders proportional to the respective numbers; due care should therefore be taken in searching the slide lest amidst the many the few be missed.

In the study of the bacteria of the respiratory tract skill in the technique of the following staining methods is essential: Gram's; 
Ziehl-Neelsen's with Spengler's modifications, Leishman's or Giemsa's; Muir's (for capsules) ; Van Ermengen's or Muir-Pitfield's (for flagella). Satisfactory results with each of these should be secured by adopting the following procedures :

Gram's method.-Materials required: Saturated alcoholic solution of gentian violet ( 5 grms. in IOo c.c. of 90 per cent. alcohol), Lugol's solution of iodine (iodine I grm., potassium iodide $3 \mathrm{grm}$., distilled water 300 c.c.); saturated aqueous solution of aniline oil, absolute alcohol, I per cent. aqueous solution of neutral red.

Procedure.-A thin uniform smear on a clean slide is allowed to dry in the air, fixed by passage three or four times through the Bunsen flame, while warm flooded with the aqueous solution of aniline oil which is filtered at the time; the alcoholic gentian violet is then allowed to drop upon the slide, one drop at each quarter inch; this ensures proper mixture of stain and mordaunt, which is allowed to remain for between two and three minutes; it is then poured off and the slide washed under the tap. The preparation is now covered with Lugol's iodine solution for fifteen seconds, washed under the tap, and flooded with absolute alcohol, which is replaced by fresh alcohol after two or three minutes. After a like interval the smear is again washed with water, held slightly aslant, and absolute alcohol dropped upon it; when no more stain appears in the washings decoloration is complete. Wash again with water, pour on the slide the aqueous solution of neutral red, leaving it on for sixty seconds in cold weather, for forty-five seconds in hot, wash with water, dry gently between folds of filtered paper, and then over the Bunsen flame. Those bacteria which retain a violet colour are said to be Gram-positive, those which lose it and assume a light red from the neutral red are said to be Gram-negative. Most varieties of bacteria are definitely either Gram + or Gram -, but a few, such as Micrococcus paratetragemus and some strains of $B$. coli occurring in urine are not definitely either one or the other and may be called Gram \pm . In a few other instances certain strains of a bacterium retain Gram's stain more or less definitely, while those which do not yet fail to assume the neutral red; some strains of the tubercle bacillus are in point.

Ziehl-Neelsen's method.-Materials required: Carbol-fuchsin (basic fuchsin I grm., carbolic acid crystals 5 grm.; dissolve in Ioo c.c. distilled water and add Io c.c. absolute alcohol), i5 per cent. aqueous solution of nitric acid, absolute alcohol, I per cent. aqueous solution of toluedene blue.

Procedure.-Fix film by passage or three four times through Bunsen flame, place vertically in staining dish (to prevent deposition of stain), filter the carbol-fuchsin which has been heated to about $40^{\circ} \mathrm{C}$. into the 
dish till slide is quite immersed; place the whole in incubator for five to ten minutes, take out the slide and wash well in water, immerse in I5 per cent. nitric acid for fifteen to twenty seconds, wash in water, again place in acid, wash with water, then with absolute alcohol till no more stain is discharged, again with water ; finally counter-stain for one to two minutes with I per cent. aqueous toluedene blue, wash well under the tap, blot lightly and dry. The bacteria, such as the tubercle, smegma, lepra, and Timothy-grass bacilli which retain the carbol-fuchsin stain after this procedure are said to be acid-fast. In as much as the presence of scanty numbers of tubercle bacilli in a specimen of sputum may be detected only with considerable difficulty when this procedure is strictly followed, various modifications of $Z$ iehl-Neelsen's method have been devised. Some of these are concerned with the actual staining processes, others merely with the preliminary treatment of the sputum; under the former head fall Spengler's methods I and II, under the latter the antiformin and pepsin methods.

Spengler's method No. I.-The smear is allowed to dry in the air, then covered for thirty to sixty seconds with I per cent. aqueous solution of caustic soda: this dissolves the mucus, but certainly does not seem to displace the bacilli; the caustic soda is poured off; of what remains adherent to the slide as much as possible is remored by touching with the edge of filter-paper, the smear is then dried best in the incubator or very cautiously over the Bunsen flame, corered with Loffler's methylene blue for two to three minutes, washed well with water, stained as before in warm carbol fuchsin, washed well with water, decolorised and counterstained by flooding the slide for thirty to forty-five seconds with Loffler's methylene blue to which five to ten drops of 15 per cent. aqueous solution of nitric acid have been added. The slide is then washed well with water, blotted lightly and dried carefully.

Spengler's method No. II.-Allow a thin smear to dry in the air, stain as before at a temperature of $35^{\circ}-40^{\circ} \mathrm{C}$. in carbol fuchsin, without washing flood the slide with a mixture of equal parts of absolute alcohol and of either Esbach's solution or of a saturated aqueous solution of picric acid, pour off after about five seconds, wash for a few seconds with $I_{5}$ per cent. nitric acid, flood with picric acid-alcohol; when the smear is of a light yellow colour, wash with distilled water, dry carefully, wash with 60 per cent. alcohol, then for a few seconds with I5 per cent. nitric acid, and again with 60 per cent. alcohol. Finally contrast stain with the picric acid-alcohol until the smear is well coloured, wash with distilled water, and dry carefully. This method is somewhat tedious, but very reliable, and by it forms which Spengler 
has named "splitter" are well demonstrated; these frequently appear as minute red granules, and are probably infant bacilli. By it I have frequently been enabled to detect tubercle bacilli in sputum which other methods have entirely failed to reveal.

The antiformin method has been devised not only for the concentration of the tubercle bacilli in a specimen of sputum, so that very scanty numbers may be detected with ease, but also for the obtaining of pure cultures of the tubercle bacilli direct from the sputum; it performs both ends with conspicuous success. Antiformin is a mixture of equal parts of a I 5 per cent. aqueous solution of caustic soda and the liquor sodæ chlorinatæe of the B.P. The procedure is as follows: 5 c.c. of the sputum are placed in a sterilised test-tube supplied with a wellfitting rubber cork; to this 5 per cent. of a 30 per cent. aqueous solution of antiformin are added, the tube is stoppered, well shaken till all the sputum is dissolved and a homogeneous mixture secured. It is then placed in the incubator at $37^{\circ} \mathrm{C}$. for one hour. The liquid is distributed into two sterile centrifuge tubes and centrifuged at a high speed for five minutes, the supernatant liquid is pipetted off, to the sediment in each tube 5. c.c. sterilised distilled water are added, and thorough centrifugalisation performed; this procedure is twice repeated. The final sediment is employed for the insemination of tubes of Dorset's egg medium, and for the preparation of smears which are stained according to one or other of the preceding methods-best by Spengler No. II. Personally, I add 5 c.c. of the mixture after incubation to one centrifuge tube, and 2 or 3 c.c. only to the other, making the volume up to 5 c.c. with absolute alcohol. The latter tube has a distinctive mark, and subsequent washings of the sediment are carried out with 40 per cent. alcohol. The addition of the alcohol lowers the specific gravity of the liquid, and facilitates the deposit of the tubercle bacilli; this tube cannot be used for culture purposes, but only for the preparation of smears.

The pepsin and trypsin methods depend upon the digestion of the sputum with acid pepsin or alkaline trypsin prior to centrifugalisation. In opposition to the view of their advocates I do not think they have any. advantage whatever over the antiformin method, which, in my hands as well as those of others, has proved most reliable and valuable.

Leishman's method has been already described on p. I3, and serves well to display the spirochætes, vibrios and spirilla of the mouth; if more intense staining be desired, a Io per cent. aqueous solution of carbol-fuchsin may be applied for forty-five seconds, followed by thorough washing in running water. Even in this dilution carbol-fuchsin is both an intense and diffuse stain; it must therefore be remembered that the bacteria as seen under the microscope will appear unduly large. The 


\section{ERRATUM.}

Page 20, line 13, instead of "to this 5 per cent. of a 30 per cent. aqueous solution"

read "to this 5 c.c. of a 30 per cent. aqueous solution" 

stains which give the truest pictures are Loffler's methylene and carbolmethylene blue, but unfortunately they are not of general utility; their use is too well known to require description.

Muir's method for capsules in my hands has proved as good as any. An advantage is its simplicity; a disadvantage the fact that the capsules thereby are stained a weak blue or sometimes not at all, in which case, however, they stand out as a clear zone around the bacillus. The following is the composition of the fixative and mordant: Saturated aqueous solution of corrosive sublimate 2 c.c., 20 per cent. aqueous solution of tannic acid 2 c.c., saturated aqueous solution of potash alum 5 c.c.; mix. The film is dried in the air, then fixed in the above for two minutes, washed in water, then in methylated spirit, and again in water. Stain in warm carbol-fuchsin for three to five minutes, wash in water, place in mordant for three minutes, wash in water. Stain for three minutes in a saturated aqueous solution of methylene blue, differentiate with methylated spirit-a process requiring considerable care-wash with water and dry carefully. Bacteria which form capsules as a rule do so only in the body, occasionally when cultured in milk or fluid media containing body fluids, rarely when allowed to incubate in the secretions in which they are voided; it thus follows that in warm weather when, say, pneumococci may multiply in the sputum during transit, only some of the forms will be seen to be capsulated in smears prepared from such a specimen. (Fig. I shows bacillus of Friedlander capsules stained by this method.)

Van Ermengen's method for flagella.-Inasmuch as this is a precipitation process and not a true staining one, it necessarily results in an undue enlargement of the stained elements; on the other hand, it is much the most reliable method. To secure good results it is absolutely essential that due regard be paid to the following points:

(I) New slides which have been thoroughly cleansed must alone be used for the preparation of the specimen.

(2) Young cultures grown on solid and not in liquid media should alone be employed.

(3) A small portion of the growth should be emulsified very gently in distilled water contained in a clean watch-glass, a drop of the emulsion taken up with the platinum loop and carefully led over the centre of the slide rather than rubbed upon it; the film should be allowed to dry in the air and not heated ; if too thick it should be discarded.

(4) Plenty of sensitising fluid should be available, and no portion of it should be used a second time even for the same preparation. Three solutions are required: (a) Fixing solution composed of 2 per cent. solution of osmic acid in distilled water I part, 20 per cent. solution of tannin in distilled water 2 parts; (b) sensitising solution, 0.5 
per cent. silver nitrate solution in distilled water ; $(c)$ reducing solution composed of gallic acid $5 \mathrm{grm}$., tannin $3 \mathrm{grm}$., fused potassium acetate Io grm., dissolved in 350 c.c. distilled water and carefully filtered.

The procedure is as follows: The air-dried film is placed in a bath of the fixing solution at a temperature of about $50^{\circ} \mathrm{C}$. for ten minutes, washed thoroughly with distilled water, then with absolute alcohol for four to five minutes, and again with distilled water. It is placed in the sensitising solution for thirty to forty seconds, and without washing transferred to the reducing solution until it turns yellowbrown: this usually takes between one and two minutes. The specimen is then transferred to a bath of fresh sensitising solution until it turns brown-black; this usually takes only a few seconds. It is washed with plenty of distilled water, very carefully dried with filter-paper, then over the Bunsen flame. This method is particularly useful for the demonstration of spirochætes, spirilla and vibrios, but, as said before, it must be remembered that the micro-organisms will appear unduly large. (Fig. 2 shows B. subtilis with flagella.) 


\section{PIATE II.}

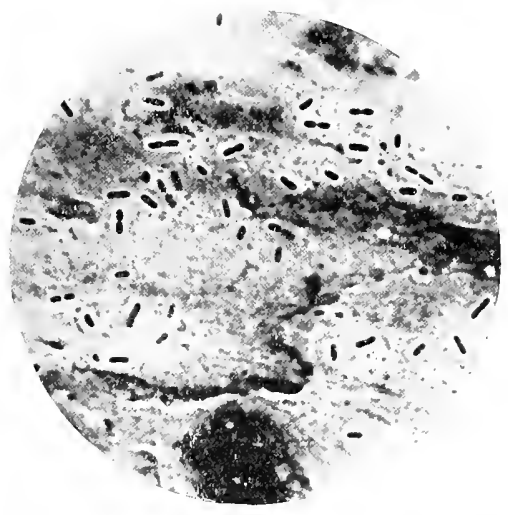

FIg. I.-Bacillus of Friedlander (capsules) and $B$. influenze in sputum. (Muir's method.) $\times$ Iooo.

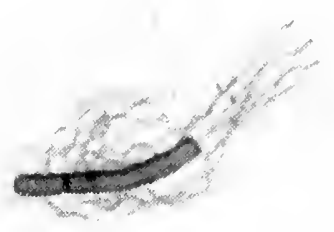

Fig. 2,-B, wubtilis, with flagella. $\times 2000$.

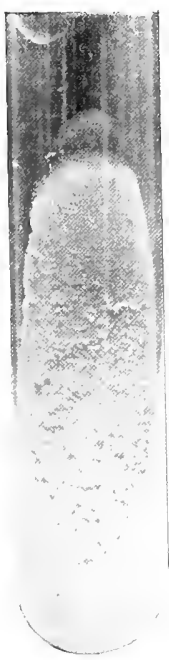

Fig. 3.-B. tuberculosis. Antiformin method. Six wetks' growth.

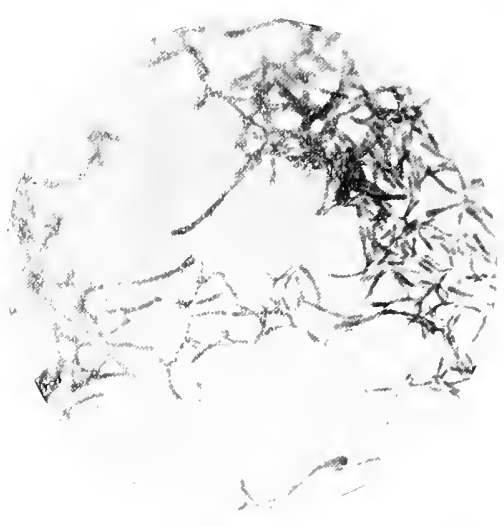

FIG. + Streptothrix from case of bronchial catarrh. Film from agar culture stained by Gram's method. × 1000 .

FIG. 5.- Streptothrix. Agar culture. (Slightly reduced.) 

CULTURAL METHODS FOR INVESTIGATING THE BACTERIOLOGY OF THE RESPIRATORY TRACT: DESCRIPTION OF THE BACTERIA WHICH MAY BE PRESENT.

To enter into a full description of all the cultural methods applicable to this study would be quite beyond the scope of this small book; there are, however, certain methods which are indispensable, and one or two others which I have devised myself for special purposes, and these may be here considered. Two preliminary points require emphasis: (I) that plate cultures have a great advantage over tube-slopes for isolation purposes. The surface is much greater and more accessible, while naked-eye appearances are more easily studied. (2) One medium is above all others indispensable; if cultures upon this be not made, then the investigation is vitiated and worthless from the outset. I refer to blood-agar, and for its preparation human blood and not that of animals should be employed. For two years my assistant and self have furnished all the blood we have required and never have we had a contaminated plate. The procedure we have adopted is as follows: I2-I5 c.c. of sterilised 5 per cent. sodium citrate are placed in a sterile tube; a capillary pipette is made from a piece of glass tubing $\frac{5}{16} \mathrm{in}$. in diameter, sterilised and fitted with a strong rubber teat. The tips of the middle fingers of the left hand are sterilised with 20 per cent. lysol, which is washed off with absolute alcohol. The finger tip is flexed, and with a surgical needle one or two punctures are made in the middle of the terminal joint. The blood should flow freely, and is taken up by means of the pipette, into which a little of the citrate solution has been already taken. About 2 c.c. of blood should be furnished by the one finger and are sufficient for one tube of citrate solution. To Io c.c. of 2 per cent. melted agar (+ Io Eyre's scale) at $60^{\circ}$ C. about I'5 c.c. of the blood citrate solution are added, and well mixed with the agar by the rotation of the inclined tube between the palms. The mixture is then poured quickly into a sterilised Petri dish of $3 \frac{3}{4}$ in. diameter. No lump of unmelted agar should disturb the surface of the plate. About eight 
plates can thus be prepared from one finger tip. Any effort to conserve the agar is to be strongly deprecated, for frequently plates require three days' incubation, at the end of which time the unduly thin plate will have so dried in the incubator as to be ill-adapted for bacterial growth; the above quantity, ro c.c., is the least which should be used for a plate of $3 \frac{3}{4}$ in. in diameter.

Other media which are necessary for the more or less complete study of this question are:

Agar (+ Io Eyre's scale).

Serum-agar (blood serum one part, 2 per cent. agar three parts).

Acid-blood agar (blood agar made as above except that to the agar [+ Io Eyre's scale] I per cent. hydrochloric acid has been added).

Dorset's egg medium (take fresh hen's egg, sterilise shell, break, mix yolk and white thoroughly, add distilled water to 25 per cent., pour into sterile test-tubes one inch in diameter, avoiding all bubbles, slant, heat in inspissator at $85^{\circ} \mathrm{C}$. for half an hour on each of three successive days).

Peptone broth-

Peptone water ( 0.5 per cent. peptone solution).

Sugar media $\left(\mathrm{O}_{5} 5\right.$ per cent. peptone water to which 2 per cent. of the various sugars has been added; of these it is well to have dextrose, levulose, saccharose, lactose, maltose, galactose, mannite, dextrin, sorbite, dulcite, inulin).

Acid-blood-peptone broth, i.e. Io c.c. peptone broth, I c.c. blood citrate mixture, to which have been added 0.5 per cent. of lactic acid and 0.5 per cent. potassium tartrate (for mouth organisms).

Special media such as MacConkey's are at times useful, and a contrivance for anaërobic incubation may be necessary.

The preliminary examination of stained smears will enable the decision to be made as to what media are to be employed, the use of a blood agar plate being obligatory: it will also indicate how much material is to be employed for insemination purposes. As a rule the amount of secretion taken up by a platinum loop one tenth of an inch in diameter will suffice; with this the blood agar plate is lightly streaked, the last stroke being made parallel to, but at the margin of the plate opposite to the first. In this way discrete colonies are certain to be secured; if they develop too thickly on the first few streaks they will be sufficiently separate on some of the subsequent ones. The plate is incubated at $37^{\circ} \mathrm{C}$. for twenty-four hours and examined by direct observation and by means of stained films. It is then returned to the incubator for another twenty-four hours, for some bacteria such as $B$. influenza, B. Bordet-Gengou, may have developed but slightly by the end of the first day, but will be easily seen by the end of the second. 
Subcultures may be made upon plates or slopes. Upon blood-agar the following varieties of bacteria will freely grow: Staphylococci, streptococci, pneumococci, B. influenza, B. Bordet-Gengou, B. KochWeek̄s, B. diphtheria, B. Hoffmanii, B. xerosis, B. septus, M. catarrhalis, $M$. paratetragenus, $M$. tetragenus, B. of Friedlander, B. proteus, B. pyocyaneus, B. coli, and B. typhosus; in fact all the pathogenic bacteria of the respiratory tract with the exception of the tubercle bacillus, lepra bacillus, streptothrix actinomycosis, Spirochata pallida, and the vibrios, streptothrices, spirochætes, and fusiform bacilli associated with pyorrhœa alveolaris-the artificial cultivation of all these is not yet possible; to those which can be cultured the following methods are applicable:

The tubercle bacillus. - The sputum having been treated by the antiformin method as already outlined, several loopfuls of the final sediment are streaked upon the surface of one or two tubes of the above-mentioned Dorset's egg-medium; after twenty-four to fortyeight hours' incubation at $37^{\circ} \mathrm{C}$. the cotton plug is flamed, pushed down the tube, and the orifice fitted with a rubber cap in order to prevent undue evaporation of moisture. After two to three weeks' incubation, pin-head colonies of the tubercle bacillus will be visible in the great majority of cases in which this organism is present (Fig. 3).

The streptothrix actinomy'cosis. $-\mathrm{By}$ far the best medium for the growth of this organism is the hydrochloric acid blood-agar. After forty-eight to seventy-two hours' incubation there will be a strongly acid odour like acetic acid, the colour of the medium will be turned brownish instead of blood-pink (from the formation, I believe, of methhæmoglobin), and small colonies, white in colour, horny in consistency, and crinkled on the surface will be found strongly adherent to the surface; after another day or two's incubation they seem to have actually eaten their way into the agar.

The vibrios, streptothrices and spirochætes of the mouth and sputum are peculiarly difficult to grow; the most useful medium is the blood peptone broth with lactic acid and potassium tartrate above referred to. With this I have at times secured abundant growth, sometimes after anaërobic, sometimes after aërobic incubation, sometimes after only six hours' incubation, sometimes after forty-eight hours. The products of growth are intensely toxic. Very occasionally pure growths can be obtained upon agar or blood agar from this blood broth (see Figs. 4 and 5 ).

When pure cultures of each of the bacteria seen in the smears, or of as many of them as can be induced to grow, have been obtained, the study of their behaviour on the special differentiating media which appear to be indicated may be pursued. The characteristics, morphological and cultural, which I have found of the greatest value for differentiation purposes will be referred to briefly in the following section. 
The Tubercle bacillus may very rarely require to be differentiated from the lepra bacillus, the smegma bacillus, and the B. Phlei (Timothygrass bacillus), and the Mycobacterium lacticola; of these the first only is of importance in diseases of the respiratory tract inasmuch as 40 per cent. of lepers are said to die from tuberculosis. The presence of vast numbers of acid-fast bacilli within the typical leprous cell is suggestive of leprosy, but fails to disprove the possible simultaneous presence of the tubercle bacillus. Lepra bacilli do not grow, except in subcultures, commonly or with any freedom upon Dorset's egg-medium, hence the appearance of copious growth in three to four weeks thereon is almost proof positive of the presence of tubercle bacilli. If no growth be obtained, the inoculation of a rabbit and a guinea-pig with some of the materies morbi may be necessary to confirm the presence of the tubercle bacillus. For recent work upon the cultivation of the lepra bacillus the reader must be referred to the writings of Clegg (Philippine Journal of Science, December, I909, p. 403) ; Williams (supplement, Indian Med. Gazette, May, IgI I ; Brit. Med.Journ., December I6th, I9II, p. I58), Rost, Twort, and Bayon (Brit. Med. Journ., November IIth, I9II, p. I269). Once the lepra bacillus has been obtained in pure culture it is said by Clegg to grow readily upon any of the ordinary laboratory media, growth upon glycerine-agar or Dorset's egg-medium being so profuse in three days that the whole surface may be covered with a heaped-up moist, creamy growth, which can be readily detached and emulsifies easily in salt solution.

The main problem which confronts us in regard to the B.tuberculosis is the determination of the type to which the given specimen belongs; is it of human or of bovine origin? The utility of Spengler's staining methods for this end I am unable to confirm. Growth upon Dorset's egg-medium affords no indication. Agglutination tests have no value. Inoculation tests have a distinct value, but are difficult to carry out, and facilities for their performance not easy to obtain. The growth in broth and determination of the acid or alkali formed at weekly intervals (Theobald Smith's method) is the best available test, but is, again, by no means easy to perform. In the case of the human strain the medium will remain acid throughout, whereas in the case of the bovine strain, after a preliminary rise in the acidity an alkaline reaction will finally appear. In short, to any but the specialised laboratory worker upon the tubercle bacillus differentiation is a practical impossibility. The reflection that in a certain small percentage of cases mixed infection by both types exists will not excite unduly the enthusiasm of the already busy man. How the difficulty can be obviated I will indicate later when I come to section IV (8). 
The Streptothrix actinomycosis. - This term is a generic one comprising many members. That transmission of infection by the Actinomyces bovis to man from horses or oxen does occur is certain, whilst infection without such an intermediary direct from barley is also possible. Streptothrices of various kinds are exceedingly common in the infected foci of pyorrhœea alveolaris, and to their ætiological significance but scant attention has been directed. Upon three occasions lately I have isolated a streptothrix from the sputum in cases of acute bronchial catarrh (Figs. 4 and 5), while an organism somewhat similar to mine but grouped as a leptothrix has been described by McDonald (Joum. Path. and Bact., 19o8, p. 447) as the probable cause of a number of cases of cerebro-spinal meningitis.

The relationship of these various organisms to the common Streptothrix actinomycosis bovis is undetermined. Streptothricial disease of the lung in man is probably much commoner than is supposed to be the case, the greater proportion of instances being missed for these reasons: (I) The organism is difficult to cultivate; (2) the typical yellow granule if detected in the sputum may be mistaken for a food-particle or for a fragment of a plug discharged from a follicular tonsillitis and examination be neglected. These plugs are by no means uncommon in sputum from such cases, and on examination may be found to contain numerous streptothricial filamentsthe clubs and spherical bodies (gonidia) and arrangement of the colonies typical of the true ray fungus will however be lacking, while cultural tests will assist in the discrimination. The discharge of yellow granules in the sputum from a case presenting obscure pulmonary symptoms should always awake suspicion of an actinomycotic or streptothricial infection.

The Spirochata pallida is hardly likely to make its appearance in the sputum or nasal discharge; on the other hand, its presence may be anticipated in smears prepared from scrapings taken from ulcerated surfaces of the respiratory tract, and it will be necessary to discriminate it from the common Spinchata refringens of the mouth. Space forbids me entering into this question, and the reader must be referred to any one of the numerous monographs which have appeared of late upon this subject.

The staphylococcus (albus, aureus, citreus, candicans) is too well known an organism to require any detailed description. It is the commonest inhabitant of the upper respiratory tract under ordinary conditions; in the pathological state it tends, if anything, to be displaced by the new invaders; to estimate its significance in disease is, therefore, no easy matter. Its rôle in pulmonary phthisis will be referred to fully in Section IV (8) (a). In diphtheria of the throat it is fre- 
quently present, but the fact that spraying with living cultures of the Staphylococcus aureus has been strongly advocated by several observers as a most useful means of destroying such diphtheria bacilli as persist during convalescence may be taken to indicate that it exercises anything but a prejudicial action in this condition. As secondary infection in lupus, syphilis, leprosy and tuberculosis of the upper passages it is well recognised as playing an important part.

In some cases of rhinitis caseosa which I have examined it would appear to have possessed an ætiological significance, but in the various catarrhal infections of the respiratory tract it apparently is not concerned excepting in cases of empyemata of the accessory spaces, and here but rarely.

The pigment formation is a variable property, and it is by no means certain that the aureus is incapable of transformation into the albus. The term "citreus" is for all practical purposes a perfectly useless one and is best abandoned. The Staphylococcus, or better-termed Micrococcus candicans, is probably a distinct and always non-pathogenic variety, possibly identical with the Micrococcus urea; colonies on gelatin or agar are round, moistly shining, porcelain-white and slightly elevated. Microscopically they are about $I^{\cdot} 2 \mu$ in diameter, i.e. about half as large again as the ordinary staphylococcus. Usually they present a dividing line in the centre, and are Gram-positive.

The streptococcus is an exceedingly important group in catarrhal diseases of the respiratory tract; its importance is far beyond that which would be gathered from any publication upon the subject. The numerous attempts which have been made to divide and classify the various members of the group have failed utterly, nay, more, they are intrinsically misleading. To call a certain strain a Streptococcus salivarius or Streptococcus mucosus was synonymous, until very recently, with attributing it with total lack of pathogenicity; nothing could be further from the truth, and I would suggest, except in purely academic discussion, the abandonment of all such terms as "salivarius," "mucosus," "facalis," and "viridans." At the same time I must admit that the study of many scores of different strains has left me unable to suggest a better classification. The discrimination between the pneumococcus and some strains of mouth streptococci is by no means easy. It is stated that incubation with pure ox-bile for thirty minutes will result in the lysis of all varieties of the streptococcus but the pneumococcus and the so-called Streptococcus salivarius.

It is also stated that the Streptococcus salivarius may possess capsules like the pneumococcus. The sugar reactions are so variable as to be almost devoid of value, and in the pathogenicity tests I am a total disbeliever, being unable to forget an experience when on the staff 
PLATE III.

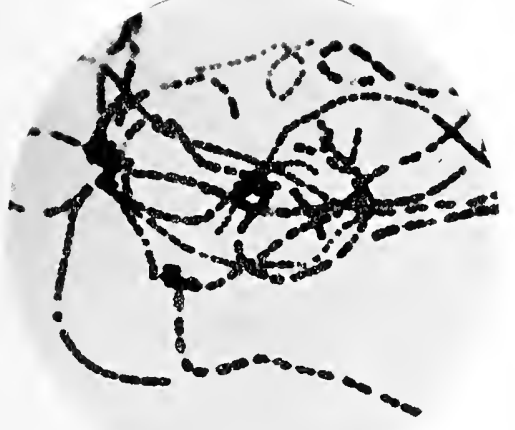

FIG. 7.-Streptococcus maximus (asthma). $\times$ IOOO.

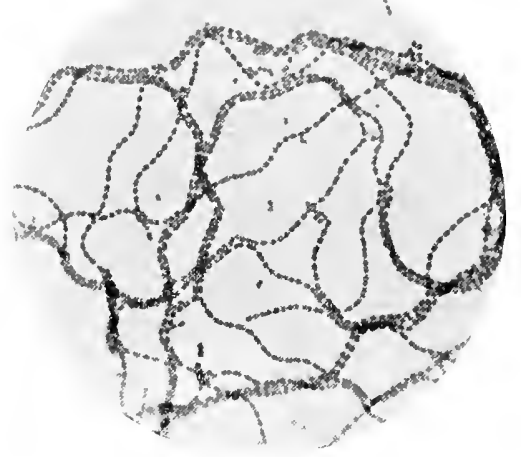

F IG. IO.-Streptococcus longus (asthma). $\times 1000$.

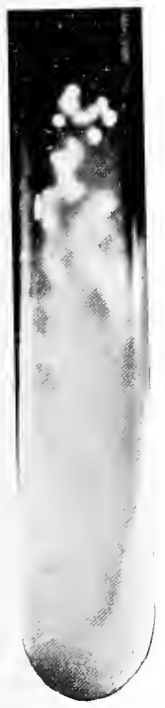

Fig. 6. - Streptococcus maximus (blood agar). Natural size.

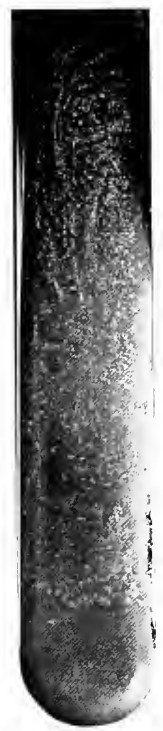

Fig. 8. - Streptococcus large from mouth (agar). Natural size.

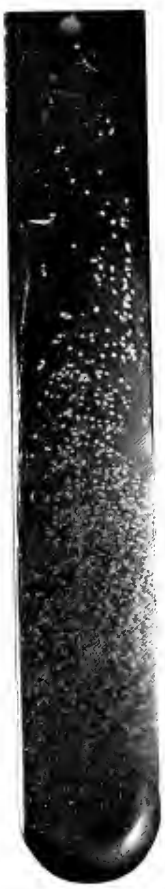

FIG. 9. 

of the Royal Eye Hospital. We had an epidemic of conjunctivitis of extreme severity and of extremely rapid course-in eighteen to twentyfour hours a complete hypopyon with ulceration of the cornea usually ensued. The lanceolate, Gram-positive, capsulated diplococci were tested on rabbits and guinea-pigs, stated to be non-pathogenic and therefore not pneumococci-could anything be more absurd? I do not for one moment wish to decry the value of close scientific observation, but so far its results as regards the streptococcus group have been most unsatisfactory. There is one variety of this organism appearing to possess constant characteristics, which is of the utmost importance in certain pathological conditions of the respiratory tract, and which, probably owing to the fact that it is highly hæmophilic and refuses to grow on agar alone, appears to have escaped recognition; for reasons which will appear I have called it Streptococcus maximus. Frequently in the sputum from cases of bronchitis and asthma there may be seen long chains of a very large streptococcus, the individual members of which are about double the size of the ordinary Streptococcus pyogenes longus from an abscess. By Gram's method they stain diffusely, decolorising with a certain amount of ease, so that some members of the chain may be quite Gram +, others Gram \pm , and yet others Gram-. Fig. 7 is an excellent reproduction by Dr. Ralph Vincent of this organism; it will be noticed that here and there is an element which has failed to retain the Gram stain. The chains may be composed of many individuals, 20, 40, Ioo, or even 200 , the average being 40 to 60 . Occasionally there may be obtained from the mouth a strain which microscopically closely resembles this one, but culturally is entirely distinct; in broth it forms a compact sediment, which on being shaken up speedily settles again to the bottom, grows readily on agar (Fig. 8), on blood agar forms discrete white colonies no larger than a small pin's head (Fig. 9), but readily visible by reflected or transmitted light. In the case of Streptococcus maximus peptone broth remains clear, a slimy deposit forming, which on being shaken up shows its viscid nature. On agar it very rarely shows any growth at all, on blood agar it grows with the utmost ease; on plates sown not too thickly colonies may attain a diameter of well over $\frac{1}{8}$ in. ; they are round, almost colourless, dew-droppy in appearance, slimy, and tend to coalesce. On blood agar slopes the colonies are difficult to see except with oblique illumination. Dr. Vincent has had great difficulty in securing a photograph of the growth, but finally, by deft manipulation of the illumination, secured the picture seen in Fig. 6. An excellent idea of the nature of the growth may be formed if it be remembered that the colonies are perfectly colourless when viewed by natural reflected light; 
the size of the colonies and their tendency to coalesce are well shown. The above characteristics suffice amply to identify this Streptococcus maximus, and to differentiate it from the large streptococcus of the mouth. In other cases, especially of asthma with scanty viscid mucus, there is frequently to be seen a totally different streptococcus; both in smears and cultures it forms chains of exceeding length which may traverse half a dozen fields of the microscope (see Fig. Io). In size the individual members of the chain show no variation from the ordinary Streptococcus pyogenes longus, and I have discovered no method of differentiating between the two.

For the rest I can merely say that we may have streptococci possessing only two, six, eight, a dozen or twenty members in a chain, streptococci possessing IOO or 200 in a chain ; we may have streptococci which on blood agar turn the blood green or turn it brown, hæmolyse or do not hæmolyse, which form colonies which are dry or moist, white or almost colourless, which in broth form flocculi, a uniform haze, or dense deposit; all these characteristics mean little or nothing in the present state of our knowledge. The fact remains that each and every one may under suitable conditions become pathogenic to the human subject in a greater or less degree, and there is no laboratory test which will settle the question whether in the particular case under review they are concerned in the process of disease or are not concerned in it. It is experience and a general review of the whole bacteriological flora which will guide aright, and the results of vaccine treatment which will confirm or refute the accuracy of the deductions. For the purposes of this paper the streptococci, then, will be divided merely into these groups-maximus, longus, and brevis.

The pneumococcus, as seen in stained preparations of the secretions, is typically a Gram-positive, capsulated, lanceolate coccus, usually occurring in pairs, occasionally in short chains of four to six members (Fig. II). The best medium for its cultivation is human blood-agar, upon which it appears in twenty-four hours as minute, round, transparent, dew-droplike colonies, thicker at the periphery than in the centre so that they present a ring-like appearance; usually they hæmolyse the medium so that there is a clear area around each colony; sometimes they also appear to turn it green, a sign, some observers maintain, of their high virulence; sometimes again they appear to turn the medium brown (Fig. I2).

Stained films of such a growth show Gram-positive lanceolated cocci, usually arranged in pairs, sometimes in short chains'of four, six, or eight members (Fig. I3).

In broth a slight uniform turbidity is produced, the addition of a little blood to the broth increasing the amount of growth; a slight dust-like deposit may settle to the bottom of the tube. If a hanging 
PLATE IV.

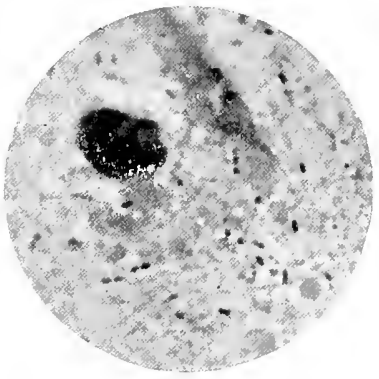

FIG. I I.-Pnenmococci in sputum. (Gram's stain.) $\times$ 1000.

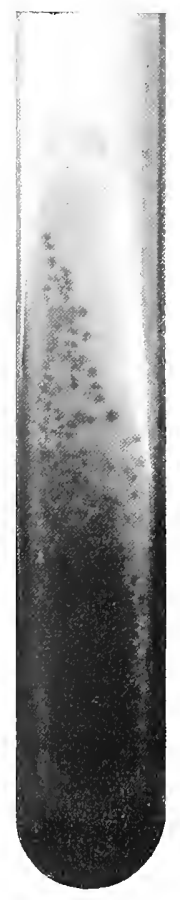

FIG. I 2.-Pneumococcus. (Blood agar) Natural size.

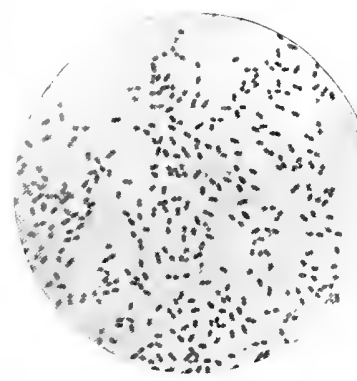

FIG. I3.- Pneumocorcus.

(Gram's stain.) $\times 1000$

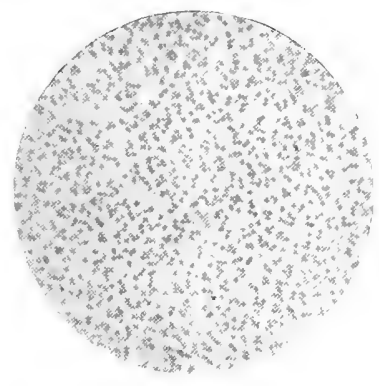

FIG. 14.-B. infuenze (Carbolfuchsin.) $\times$ rooo.

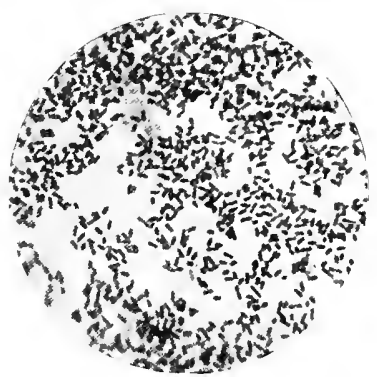

Fig. I 5.-Koch-Weeks' B. (Carbolfuchsin.) $\times$ 1000. 

drop or stained film be examined numerous chains of four, six, or eight individuals will be observed.

The fact that growth does not occur on gelatine or agar at room temperatures assists to differentiate the pneumococcus from some strains of the short mouth streptococcus; the distinction, however, is not an absolute one, for some of these latter also fail to grow under like conditions.

I have already referred to the failure of inoculation tests to prove or negative the fact that a given organism is a pneumococcus. With those who hold that some strains of the mouth streptococcus are capsulated and behave like the pneumococcus when incubated with ox-bile I am unable to agree, and consider that they were dealing with true pneumococci of low virulence, and that it is best to regard all capsulated, Gram-positive, lanceolate diplococci which give the characteristic colonies on blood-agar as true pneumococci, no matter what their fermenting powers or pathogenic properties in animals.

The Bacillus infuenze, B. of Koch-Weeks, and B. of Bordet-Gengon are very closely allied organisms, nay, further, the former two are probably identical, although the fact that with the acute conjunctivitis due to the Koch-IVeeks' bacillus it is very rare to find a concurrent respiratory catarrh is rather against this view ; microscopically they are indistinguishable (Figs. I4 and I5).

The Bacillus influenza and B. Koch-Weeks only grow in or on media containing blood or hæmoglobin, viz. blood broth or blood-agar. If a thin layer of blood-broth be inseminated and incubated at $37^{\circ} \mathrm{C}$. for twenty-four hours, delicate white flocculi are to be seen; on blood-agar after twenty-four hours' incubation at $37^{\circ} \mathrm{C}$. pin-point colonies, round, perfectly colourless and moist, are formed; after forty-eight hours these may attain a diameter of $\frac{1}{16}$ in. and by reflected light are perfectly colourless, by transmitted light grey to whitish.

Microscopically they consist of very narrow short rods, $0^{*} 4 \mu$ broad, $I \cdot 2-I \cdot 5 \mu$ long, which fail to retain the stain by Gram's method, but take up the neutral red. Weak carbol fuchsin shows them up most clearly, but from its intense and diffuse action causes them to appear unduly large (Figs. I4 and I5). Sometimes they appear in pairs, sometimes short filaments are formed, this characteristic being peculiar to certain strains of unusual luxuriance of growth which are, perhaps, pseudo rather than true influenza bacilli. In the secretion they often appear inside the cells, and yet more frequently are aggregated around the pulmonary or polynuclear cells. The Bacillus Bordet-Gengou (of whooping-cough) differs from the B. influenza in being slightly larger, ovoid, more regular in size and in exhibiting polar staining. In cultural properties it also differs, growing best upon glycerine-potato- 
blood-agar, and on serum agar, upon which latter medium the influenza bacillus grows with difficulty. Upon the former medium after twentyfour hours' incubation at $37^{\circ}$ pin-point colonies may appear ; these will be $B$. influenza. After forty-eight to seventy-two hours much larger and more vigorous colonies which do not hæmolyse the blood medium may be seen. These are colonies of the bacillus of Bordet-Gengou. In subcultures they form a dense, grey, glistening, and very vigorous growth of discrete colonies. The tendency of the B. influenwe, on the other hand, is to die out rapidly when sub-cultured. The addition of various bacterial toxins, as of the $B$. coli, pneumococcus or staphylococcus to the blood agar will frequently result in an extraordinary profuse growth of the B. influenza (vide Vaccine Therapy, 3rd edition, pp. I74, I75). It may be noted that in the majority of cases of whoopingcough the B. infuenza appears to be present along with the Bordet's bacillus. The significance of this fact in the treatment of whoopingcough by means of vaccine is obvious.

The Bacillus diphtheria (Klebs-Loeffler), B. of. Hoffmann, B. xerosis and $B$. septus constitute the group of so-called diphtheroid bacilli. While it is true that the first of these is usually associated with the formation of a membrane, be it in the throat, larynx, nose or eye, it yet happens that it may be resident in the throat in the absence of any membrane, either persisting for months after the subsidence of all acute symptoms of a diphtheritic attack, or maintaining a constant saprophytic existence there, at no time causing any symptoms, but being a continual potential source of danger either to its host or to others to whom it may be conveyed; such individuals are known as "carriers."

The bacillus of Hoffmann is a frequent cause of epidemic sore throat, not so frequent, however, I believe, as it is held to be by many observers, who may possibly have confused it with the Bacillus septus. So far as I know Hoffmann's bacillus does not invade the nose, and would not appear ever to cause an acute rhinitis.

The Bacillus xerosis is believed to be non-pathogenic; whether it is entirely devoid of pathogenicity is in my opinion debatable, but whether it be or not, its pathogenicity is certainly very low. It is commonly found associated with other bacteria in chronic inflammatory conditions of the ocular and urethral mucous membranes. It might therefore be anticipated that it would frequently be found resident on the nasal mucosa, especially in those suffering from conjunctival inflammation; such, however, I have not found to be the case; the only diphtheroid commonly found in the nose is the Bacillus septus.

The Bacillus septus is associated with catarrhal affections of the nose, naso-pharynx, and throat. Only once have I found it in the sputum 


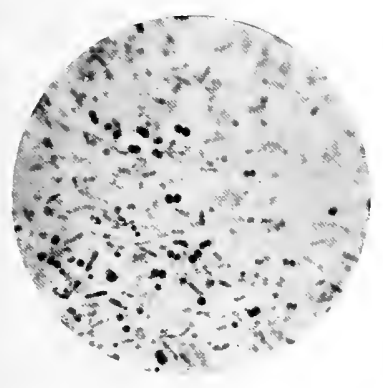

F $1 \mathrm{G} \quad 16 .-B$. septus and.$M$. catarhalis. $\times 1000$.

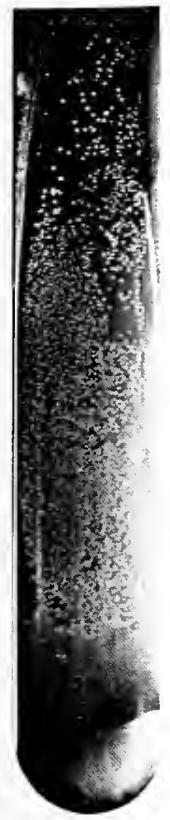

FIG. I7.-B. diphtheria, twenty-four hours. Natural size.

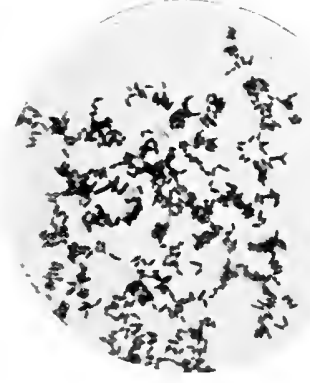

FIG. 20.-Diphtheria bacillus, twenty-four hours. Gram.) $\times 1000$.

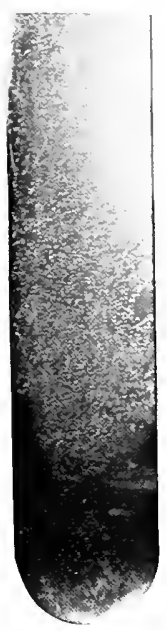

FIG. IS.-B. xerosis, twentyfour hours. Natural size.

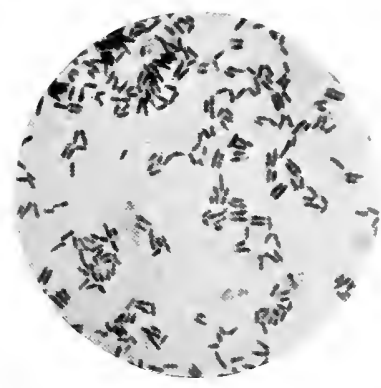

FIG. 21.-B. Hofmann, twentyfour hours. (Gram.) $\times 1000$.

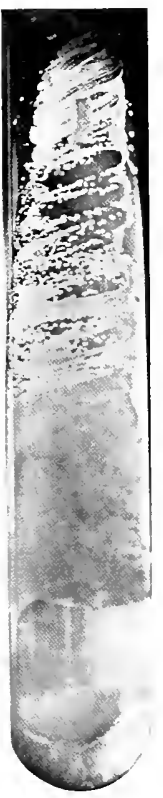

Fig. 19.-B. septus twenty-four hours Natural size. 

from a case of bronchial catarrh (see Fig. I6, a smear of the sputum of this case; the deeply-stained cocci are $M$. catarrhalis, the faintly stained rods $B$. septus). This was a long-standing infection, the expectoration was profuse and very purulent-looking, although almost free from cells and consisting almost exclusively of mucus and bacteria and was most offensive; if incubated for twenty-four hours the odour was overpowering, with a suggestion of impure petrol about it. This case will be more fully referred to in a subsequent number. Upon two other occasions I have isolated from the sputum a diphtheroid bacillus which was not a member of any of these four groups. On agar and blood agar it formed round, discrete, moist, oily colonies, the colour of which closely resembled that of honey; it emulsified with ease, but almost immediately agglutinated and refused to be again emulsified.

To enter into a detailed description of the characteristics, morphological and cultural, of the members of this family is forbidden by the scope of this paper, but it will perhaps prove of service if I tabulate the more important points of differential diagnosis (see Table I).

A knowledge of the clinical features of the case and of the source of the material will assist one whose experience of the morphology and cultural characteristics of these organisms is limited in arriving at the correct differential diagnosis.

The Micrococcus catarrhalis group.-Of all the micro-organisms infecting the respiratory tract, this is at once the largest, commonest, and most widely distributed group. In the present state of knowledge it is a term applied to all the cocci found in these parts which fail to retain the stain by Gram's method, with the single exception of the meningococcus or micrococcus of epidemic cerebro-spinal meningitis (Weichselbaum). I have isolated at least fifty members of this group differing the one from the other in some detail, morphological or cultural, and the following description must be taken as only applying strictly to the form most commonly found in catarrhal infection of the respiratory tract. It is a Gram-negative coccus, closely resembling the gonococcus in shape, and, like it, frequently growing in pairs; it differs from the gonococcus in being less definitely kidney-shaped, and on the whole larger, and in showing greater variation in size; every film will show some forms larger than the majority and decolorising with less ease; and in the fact that arrangement into tetrads is quite common, chain-formation does not occur in what is to be regarded as the typical Micrococcus catarrialis, aggregation into clumps is the rule. The following table will help in the differential diagnosis (Table II).

Whether the smaller pseudo-catarrhalis is ever pathogenic is doubtful, and it may be given as a general rule that the larger the individual 


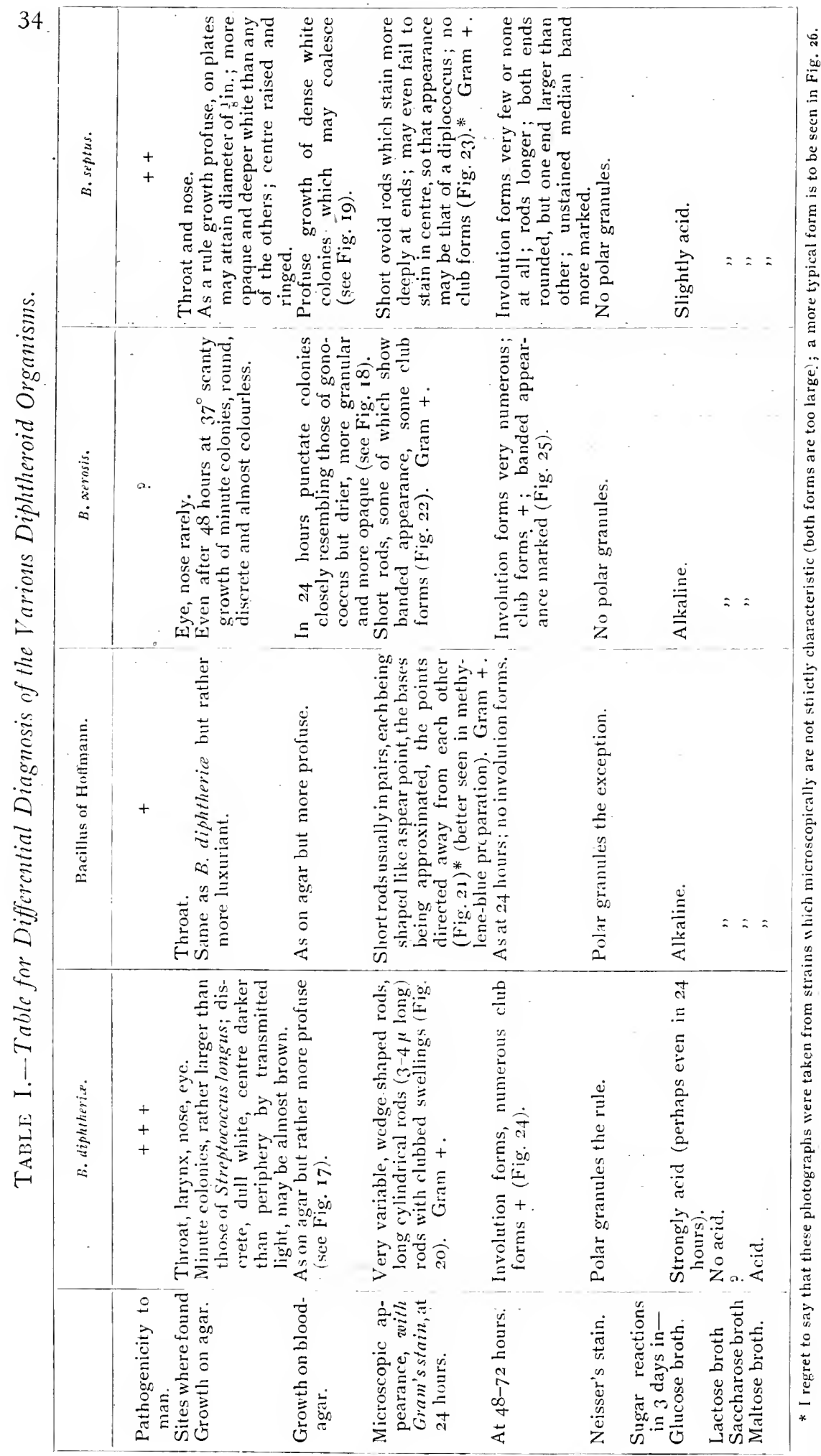


PLATE VI.

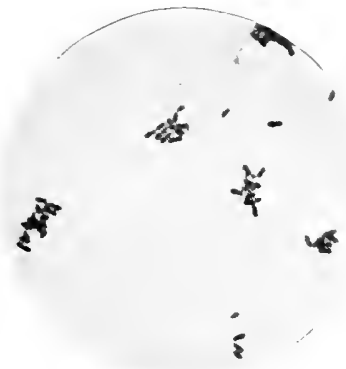

FIG. 22. - B. xerosis, twenty-four hours. (Gram.) × rooo.

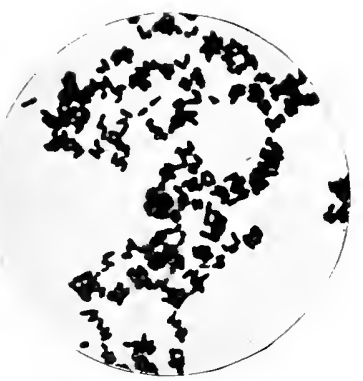

FIG. 24-Diphtheria bacillus, three days. Gram) × 1000.

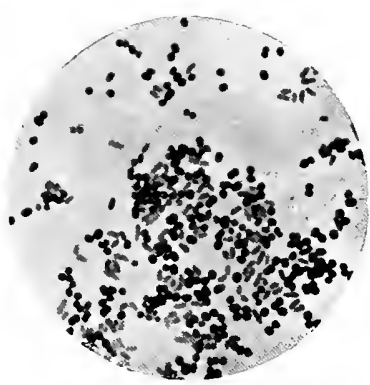

FIG. 26.-B. septus and M. catarrhalis. (Gram counterstained neutral red.) $\times 1000$.

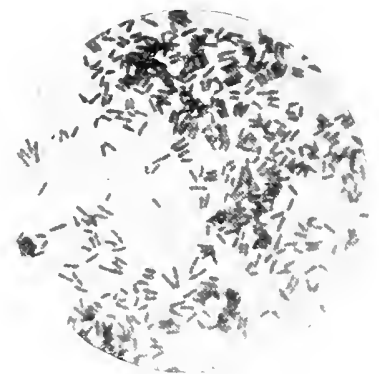

FIG. 23.-B. septus, twenty-four hours. (Gram.) × I000.

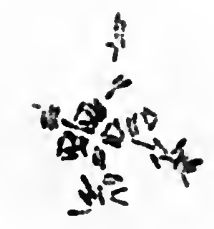

FIG. 25.-B. xerosis, three days. (Gram.) $\times 1000$.

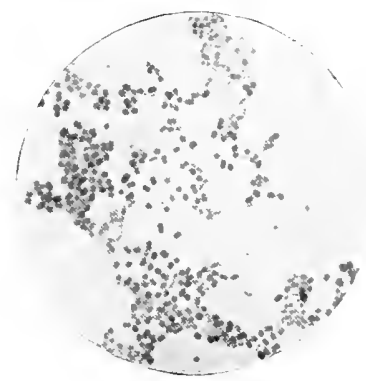

FIG. 27.-M. catarhalis. (Gram counterstained neutral red.) $\times 1000$. 



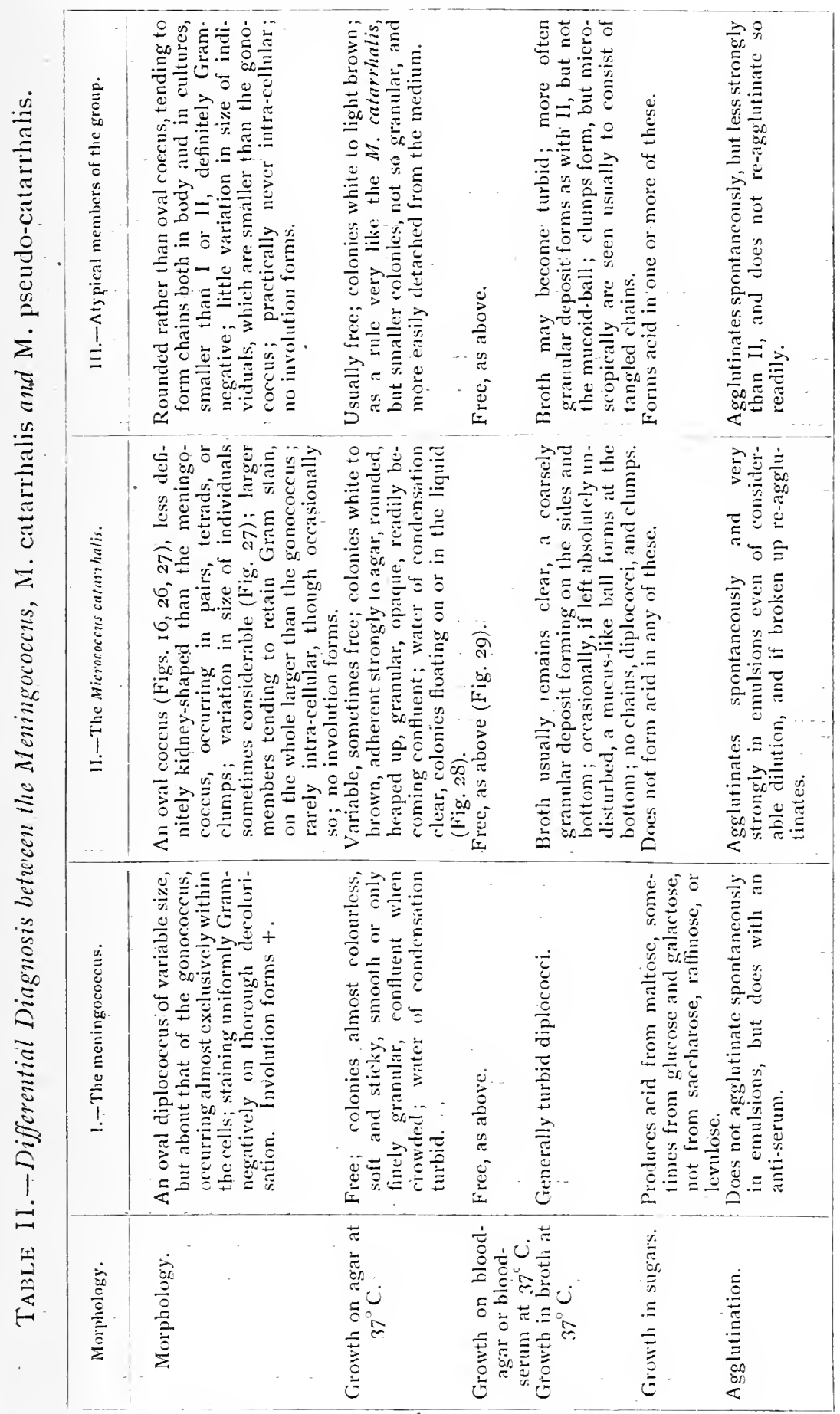


members of the M. catarrhalis the greater is the likelihood of its being pathogenic; to this statement there is, however, an exception; there is a form which morphologically is a true $M$. catarrhalis, except that it is in size rather less than two thirds that of a gonococcus (see Fig. 30). Culturally, however, it is favoured by anaërobic conditions, the colonies on blood agar then being much smaller than those of the typical $M$. catarrhalis, discrete and almost invisible instead of heaped-up, aggregated, crinkled and brownish. It appears to be strongly pathogenic, and whether it really belongs to the $M$. catarrhalis group must be left an open question.

It will be remembered doubtless against me that when discussing the streptococcus group apparent exception was taken to value being attached to exact laboratory research in this domain, and I shall certainly be exposed to a charge of inconsistency in advocating the necessity for accurate laboratory research being conducted. upon the Micrococcus catarrhalis group; it is therefore incumbent upon me to anticipate this charge and make a defence. What I really objected to in regard to the streptococcus group was not the performance of minute investigation, but the attachment of greater value to pathogenic experiments upon animals and the reactions in various sugar media than to clinical observation, a tendency which certainly has had an existence, but now happily is passing away.

The case with the Micrococcus catarrhalis group is not quite parallel. These micro-organisms so far as the respiratory tract is concerned are ubiquitous; they exist, as apparently does the streptococcus, in every mouth and throat, but they exist in far greater variety, and under far more widely divergent conditions. If in the nasal mucus of a case of acute rhinitis a Micrococcus catarrhalis be found in profusion, little doubt exists as to the causal relationship of the bacterium to the disease, and it is rare indeed to find divergent strains in such a case; in regard to the sputum, however, it is quite another matter. From practically every mouth, pharynx, and naso-pharynx in health or in disease numerous Gram-negative cocci can be recovered, and considerable variations will be discoverable, both morphological and cultural. As I have said, I have recorded about fifty strains, differing in some slight detail the one from the other. Now it will be urged that there are at least two hundred strains of streptococci; true, but I believe that each and every one of these is either pathogenic to, or capable of being roused into pathogenicity to, its host. Not so with the Micrococcus catarrhalis group. Some, nay, most of its members are probably absolutely devoid of pathogenic properties, and supposing that even half a dozen different strains are isolated from the materies morbi, we are at present without any means whatsoever of determining which strains are responsible for 


\section{PLATE VII.}

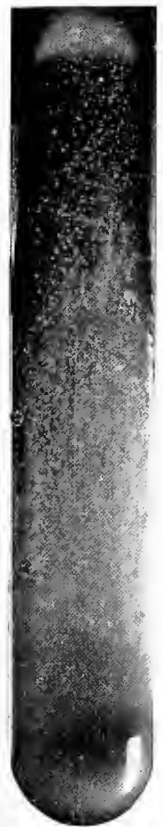

FIG. 28.-M. catarhalis (agar). Natural size.

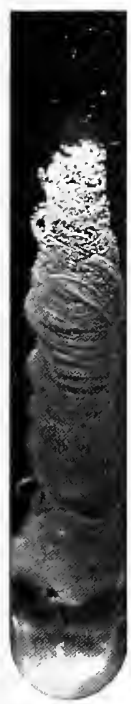

FIG. 34--Sarcina lutea. Slightly reduced.

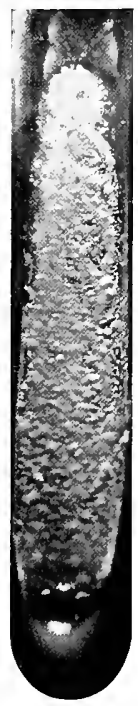

Fig. 33.-M. paratetragenus. Slightly reduced.

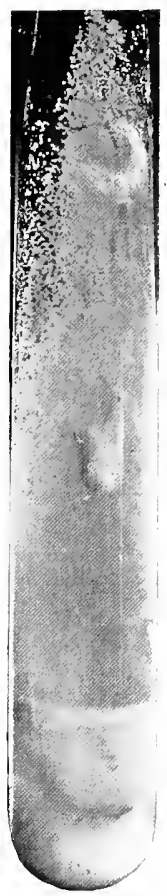

Fig. 35.-M. tetragenus. Natural size.

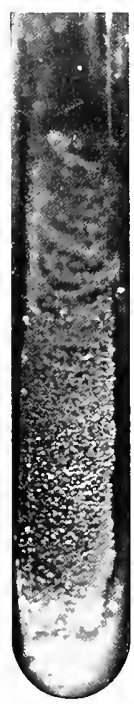

Fig. 29--M. catarrtalis (blood-agir). Slightly reduced.

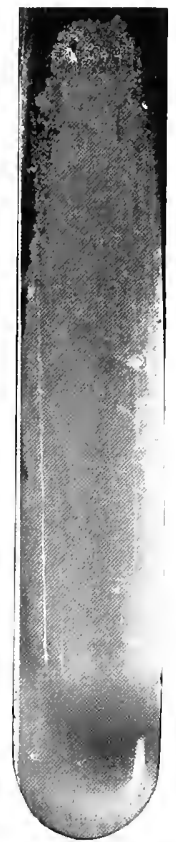

Fig. 37--Bacillus of Friëdlander. Natural size. 

the condition and which are not, of deciding which strains to incorporate in a vaccine and which to leave out. We have no alternative but to include them all. Just as I object to over-much regard being paid to the laboratory classification of a streptococcus and consequent neglect of it as a possible factor in the given pathological condition, so I regret the absence of a reliable method of differentiating the Micrococcus catarrhalis group, and our consequent inability to separate strains which are pathogenic from those which are not. Opsonic index estimations may help, but are much likely to prove of no avail; agglutination tests are inadmissible owing to active spontaneous agglutination in the emulsion.

The Micrococcus paratetragenus. - The first to ascribe pathogenic properties to this organism was Bezançon. Benham has found it in a case of pulmonary abscess, and constantly in an epidemic of colds. I also found it a causal factor in almost every case of catarrh investigated during two epidemics. The last two years it has not been in evidence; this year it is, however, again occurring in a certain percentage of cases. As its names implies, it is a micrococcus usually occurring in tetrads, but also in pairs. These are usually grouped together in a zooglœal mass. They are not capsulated, but both in sputum and in culture films the mucoid material faintly stains, giving a capsular appearance. Fig. $3 \mathrm{I}$ is a photo-micrograph of a smear of sputum showing dark masses of Micrococcus paratetragenus and more faintly stained bacillus of Friedlander. Fig. 32 shows admirably the appearance of a stained film of a pure culture. The more darkly stained kidney-shaped tetrads are seen imbedded in the faintly stained zooglœal mass. The Micrococcus paratetragenus is the best of all instances of organisms which may be styled Gram \pm . Unless decolorisation with absolute alcohol be very complete the gentian violet will be retained, feebly but definitely, so that even two minutes' staining with neutral red will fail to displace it. Strictly I think it should be regarded as a Gram-positive organism. In smears it is apt to be confused with the Micrococcus tetragemus and with the Sarcina lutea; from the former it is differentiated by its larger size and peculiar staining properties (compare Figs. 32 and 36), from the last by its smaller size, from both by its cultural properties ( $c f$. Figs. 33, 34 and 35 ).

It grows well on agar (Fig. 33; cf. Fig. 34 of Sarcina lutea and Fig. 35 of Micrococcus tetragenus), better on blood-agar. When sown thinly the colonies are white, rounded, dry, umbonate; they adhere to the medium like the Micrococcus catarrhalis, and emulsify with some difficulty; once emulsified, however, there is little or no tendency to spontaneous agglutination. Heating to $60^{\circ}-70^{\circ} \mathrm{C}$. aids emulsification, whereas with Micrococcus catarrhalis it often tends to stronger agglutination; when 
sown thickly it forms heaped-up masses. In both it produces some turbidity and a slimy deposit on the bottom.

I have found it form a small amount of acid from the following sugar media : dextrose, levulose, saccharose, galactose, lactose, and from mannitol.

The Micrococcus tetragenus has, I think, often been confused with the Micrococcus paratetragemus, which in several ways it closely resembles. It also is a kidney-shaped coccus, arranged in pairs or fours, and surrounded by a gelatinous pseudo-capsule, which stains faintly (well seen in Fig. 36). It is, however, distinctly smaller, about two thirds the size, and is definitely Gram-positive. It is, unlike the $M$. paratetragemus, a pyogenic organism, producing in laboratory animals an abscess locally and a rapidly progressing septicæmia generally.

On agar it forms, relatively to the paratetragenus, small colonies (Fig. 35), which are white, slightly elevated, shiny, and moist; the borders are at first even, later sinuous in outline. When growing on plates it gives forth an odour like glue. In broth it leaves the medium clear and forms a moderate precipitate, which on shaking rises as flocculi and then disperses.

It forms acid in glucose and lactose.

The bacillus of Friedlander group includes a considerable number of organisms presenting slight variation. In the secretions they occur usually in pairs, each individual surrounded by a wide capșule (see Fig. I, March number).

The rods vary considerably in length $(0 \cdot 5-3.5 \mu)$ and have rounded ends. They are non-motile, stain readily with any dye, but lose the stain by Gram's method and take on the neutral red (Fig. 38). They grow with extreme luxuriance upon or in almost every medium.

On gelatine and agar plates when sown thinly the colonies may attain a diameter of even a quarter of an inch. They are round, grey to white, iridescent, elevated, and slimy, so that on slopes they readily coalesce and may run down to the bottom of the tube (Fig. 37); the water of condensation becomes turbid. Deep colonies in plates are oval or whetstone shaped. Gelatine is not liquefied. In a gelatine or agar stab well-developed growth occurs, giving the appearance of a string of pearls, while on the surface an elevated growth like a nail-head is formed. On potato a thick, moist, shiny growth yellow or greyishbrown in colour rapidly develops. Growth in broth is also luxuriant, the medium becoming quite cloudy and a slimy deposit forming. Almost all the sugar media are rapidly fermented with formation of both acid and gas.

In working upon the bacteriology of the respiratory tract frequently one meets with organisms possessing characteristics varying more or 


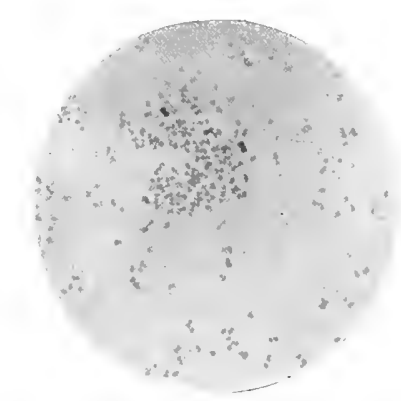

FIG. 30.-H. catarrhalis, small type. (Gram counterstained neutral red.) $\times$ I 000 .

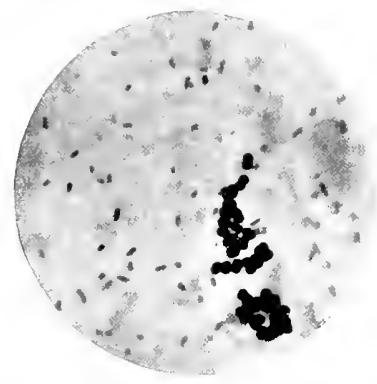

Fig. 31.-Bacillus of Friedlander and $M$. paratetrasenus in sputum. (Gram counterstianed neutral red.) × rooo.

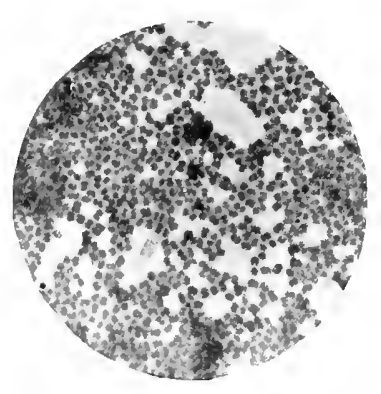

FiG. 32.-.1\% paratetragenus. (Gram countersiained neutral red.) $\times 1000$.

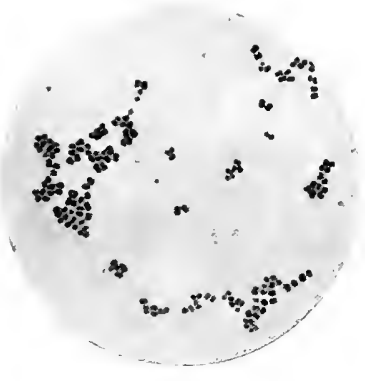

FIG. 36.- H. tetragenus.

(Gram.) × г ooo.

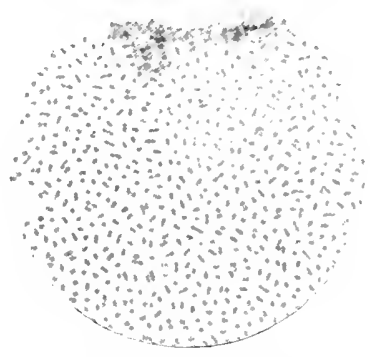

FIg. 38 - Bacillus of Friëdlander. (Gram counterstained neutral red.) $\times$ I ooo. 

less from the above. They may be capsulated or not capsulated, they may be motile or non-motile, definitely Gram -, or tending to retain the stain, they may or may not liquefy gelatine, on agar form a dry or slimy white or almost colourless growth, in a stab culture form the typical moist, heaped up, nail-head growth, or a dry, porcelain-like diffuse growth, ferment all, some or none, of the sugars.

In any one or more of these particulars variations may be seen; thus there are all gradations between the typical bacillus of Friedlander as described above and the Bacillus protens.

The Bacillus proteus vulgaris, as its name implies, is a very pleomorphic organism ; it may occur as slender rods varying in length from $\mathrm{I}$ to $+\mu$ in length, as long straight or spiral threads. It is actively motile, and does not form capsules.

Its staining by Gram's method is variable. Lehmann and Neumann have no doubt that it is Gram-positive ; other observers are equally certain that it is Gram-negative; personally I regard it as Gramnegative.

Like the bacillus of Friedlander almost any medium is suited to its growth. On gelatine it forms a delicate, grey, transparent growth, but rapidly liquefies the medium. On agar it forms grey, slimy, transparent, rounded colonies. In broth it forms considerable turbidity and abundant precipitate. Milk is coagulated and then liquefied. On potato it forms a scanty yellowish growth with a dull lustre. In the various sugar media it forms abundant gas and acid.

Alone it would appear only infrequently to give rise to pathological conditions of the respiratory tract and its adnexa; more often it is associated with other pathogenic organisms. I have isolated it in pure culture from several chronic cases of bronchial and Eustachian catarrh and middle-ear disease; as these cases have recovered rapidly upon exhibition of the autogenous vaccine its causal relationship to the conditions was more or less confirmed.

The Bacillus pyocyaneus (the bacillus of green or blue pus) is an actively motile Gram-positive bacillus, usually in the form of slender rods $(\mathrm{I} \cdot 5-6 \mu)$ long, but sometimes very short and plump, sometimes almost threadlike. It is characterised by the yellow-green or blue-green fluorescence which it produces in the media upon or in which it grows, and by the early liquefaction, at first cup-shaped, later cylindrical, which it produces in a gelatine stab. That alone it is capable of producing pathological conditions is certain; at the same time when present in such affections of the respiratory tract and its adnexa as ulcers of the mouth and pharynx, otitis media and pulmonary abscesses, it usually occurs associated with other pathogenic organisms, and the difficulty of determining its precise role is not lessened by the following 
considerations: (I) that in a given condition which is remaining more or less stationary the B. pyocyancus may be present for a few days, disappear for a time, then reappear-perhaps again to disappear of its own accord; (2) that two other bacteria which appear to be entirely nonpathogenic very closely resemble the $B$. pyocyaneus, viz. the $B$. furorescens liquefaciens and B. fuorescens non-liquefaciens. The former is indistinguishable from the $B$. pyocyanens in its morphology and cultural reactions, except that it does not coagulate milk, and is said to be non-pathogenic to animals. The latter is differentiated by its failure to liquefy gelatine.

Unless, therefore, the $B$. pyocyancus appears to be the sole invader or is present in overwhelming numbers in a characteristic pus, I feel disposed as a rule to give it a chance of spontaneously disappearing; when, however, it fails to do this, or the patient does not make adequate progress under treatment with the vaccines of its co-invaders, I feel the time has come to use its vaccine. At the same time it must be granted that if money is no object to the patient the immediate exhibition of the pyocyaneus vaccine in suitable doses, either alone or in combination with other vaccines which may be indicated, is hardly likely to have any ill-effects and may be productive of good.

The $B$. coli communis is so well known and so adequately described in all text-books of bacteriology that little need be said about it beyond pointing out: (I) That it is said fairly frequently to complicate pulmonary tuberculosis; personally I have found it rarely. (2) That it is often present in lung abscesses and in empyemata, especially if these be of long standing; as a rule it is, however, not the primary infection, and unless the discharge itself be carefully examined and plating-out be conducted with unusual care the pneumococcus, streptococcus or $B$. influenza, which constituted the primary infection, may easily be missed, and the consequent treatment with a coli vaccine lead to not entirely satisfactory results. (3) That occasionally, after abdominal operations, it may produce a diaphragmatic pleurisy or basal pneumonia, progressing later to an empyema. I have seen such ensue after fixation of a kidney, the whole abdominal cavity remaining free from active infection. (4) That in the secretions the $B$. coli often retains the Gram stain so strongly that unless the treatment with absolute alcohol be thoroughly performed the bacilli may appear to be Grampositive; the cultural tests will, however, resolve all doubt.

The $B$. typhosus I merely mention for this reason: It is, I believe, a far more frequent factor in the production of typhoid pneumonia than is commonly realised. In the sputum of such cases careful search should be made, not only for the typhoid bacillus, but also for the pneumococcus and streptococcus, for in cultures the first will overgrow 
the latter two. Vaccine treatment, in my opinion, offers much the best hope of recovery in these cases, but success is largely determined by accuracy in the diagnosis of the responsible bacteria.

The organisms associated with follicular tonsillitis and pyorrhcea alveolaris are legion in number. Some recent experiments of my own have shown what intensely toxic products are formed by some of these, while Vincent's fusiform bacillus has recently been shown to be possessed of definitely pyogenic properties. Cases have been recorded of acute dermatitis and abscess formation as resulting from bites, and from these the fusiform bacillus has been recovered in pure culture; it would also appear to be the cause of a peculiar form of sore very prevalent in some of the South Sea Islands. Strictly anaërobic conditions greatly favour its artificial culture.

Most of these mouth organisms stain but faintly with most of the dyes, and for the study of their morphology weak carbol fuchsin is to be recommended. 


\section{CHAPTER IV.}

\section{B (III). RESULTS OF OBSERVATIONS INTO THE BACTE- RIOLOGY OF THE VARIOUS DISEASED CONDITIONS OF THE RESPIRATORY TRACT.}

Any attempt at determining the precise rôle played by a given bacterium in the causation of pathological conditions of the respiratory tract is beset by numerous difficulties. The enumeration of a few only of these will help to show how real these difficulties are.

(I) As one who has been working continuously on the subject for nearly ten years I am obliged to confess that one's views as to what may be and are not causal factors have been steadily changing; an organism which one now knows beyond doubt to be concerned in a given process was not even considered seven years ago, and carefully as all records have been kept, an uneasy suspicion is aroused that observations even five years old are not as trustivorthy as one would like them to be.

(2) The results of examinations of smears of secretion are not always confirmed by the results of plating experiments; for instance a smear may show vast numbers of what appear to be $B$. infunenze, while a plate prepared from the same secretion may after even three days' incubation fail to show a single colony of that bacterium; confirmation therefore is lacking of the identity of the bacillus seen in the smear.

(3) Again, the predominant organism seen in smears may not be the predominant organism found in cultures, and even if it be granted that the former observation is more likely to give a true picture of the bacteriology of the condition than is the latter, we are faced by doubts as to whether the predominant organism necessarily is responsible for the condition rather than one of those which is present in relatively few numbers. As the result of experience I would say that in settling this question much depends upon the stage of the disease. To take one of the simplest examples, at the very beginning of an attack of acute nasal catarrh the Staphylococcus albus may alone be detected in the excreted 
mucus and in cultures of swabs from the middle turbinals; three or four days later the Bacillus septus may reign supreme to the total exclusion of all other organisms; after yet another interval of three or four days Staphylococcus albus, $B$. septus and $M$. catarrhalis may all be present, and the first or last of these three may predominate. Even more complex may be the results of observations upon the secretions in a case of acute or subacute sinusitis or bronchial catarrh.

(4) Observations, if they are to have a definite value, must be extended over a considerable space of time. As I shall show presently, the results of observations made, say, last year are very dissimilar to those of observations made five years previously; this would appear to be due to the fact that either the pathogenicity of the various organisms to man rises and wanes, or the resisting powers of the human race towards a given bacterium are exalted and depressed in turn. Due allowance must therefore be made for this in the compilation of statistics as to the relative frequencies with which the various bacteria indicated are concerned in the several pathological conditions. For instance, during the four years I905-I908 I found the $B$. influenwe in only 2.4 per cent. of the cases of respiratory catarrh which I examined, whereas during the three years Igog-IgII it was present in over fo per cent. of the cases investigated. It thus follows that to the percentage figures obtained from my own long-continued observations, either alone or combined with the results of other investigators over shorter periods, undue importance must not be attached; they have a certain value and a certain interest, but that is all.

(5) That whereas the truly acute attack is capable of differentiation from the truly chronic it is by no means easy to know under which category to place the subacute, and still less easy to know how to deal with the findings in an acute attack upon a chronic one. Of all the catarrhal organisms there is only one which, with very rare exceptions, gives rise to acute attacks alone and not to chronic, viz. the $B$. septus.

(6) That it is very rare for the most strictly localised infection to remain localised for long; the simplest nasal catarrh in the greater majority of cases is soon complicated by an infection of one or other of the sinuses; inasmuch as this sinusitis usually clears up rapidly of its own accord its presence easily escapes recognition. Again, in the latter stages of nasal and post-nasal catarrh, when the secretion has become thick and difficult to expel, forcible efforts at its expulsion result in a temporary dilatation of the Eustachian tube or tubes and consequently their frequent infection. This, again, as a rule, speedily subsides. Occasionally, however, infection persists in both instances, the sinuses or tubes becoming infected by the bacteria resident in the 
parts at the time of infection, whereas the bacteriology of the nasal or post-nasal catarrh is liable subsequently to undergo a change, secondary invaders in whole or part taking the place of the primary ones. It is therefore not necessarily to be expected that the bacteriology of the nasal mucous membrane, of the antrum and the frontal sinus will be the same in a case of extension of the infection of a chronic nasai catarrh. Or again, an acute rhinitis due to the pneumococcus may extend steadily down the respiratory tract until finally a pneumococcal bronchitis is set up, or, as perhaps is more usual with this organism, a pneumococcal laryngitis may extend simultaneously upwards and downwards, so that at the end of about five days a condition of acute rhinitis and bronchitis has superseded the primary laryngitis. The difficulty, therefore, of classifying the conditions accurately is by no means a small one. With these reservations I proceed to give a few statistics of the bacteriological findings by various observers in catarrhal conditions of the respiratory tract.

\section{(1) In Nasal and Post-nasal Catarrh.}

TABLE III.

Bacteriology of Nasal and Post-nasal Catarrh (R. W. Allen's figures, I905 to IgII inclusive).

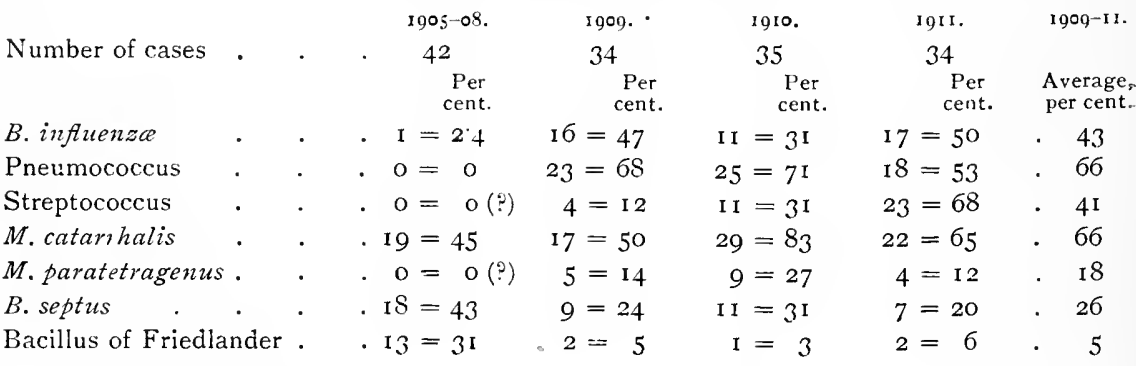

Allowing for the fact that the absence of streptococci during the period of 1905-08 was probably more apparent than real, inasmuch as I did not then recognise it as a factor in the production of post-nasal catarrh, the most striking features are the great contrast in the frequency with which the $B$. influenze and pneumococcus was found in the two periods I905-08 and I909-II. In the Lancet of February I3th, I909, p. 500, I recorded the incidence of this pneumococcal epidemic which has persisted till the present time; the influenzal epidemic began about two months later, and whereas the pneumococcus is now showing a tendency to die out, the $B$. influenza has as yet shown no such tendency. The type of concurrent complications. has, moreover, changed, neuritis and herpes zoster having been 
prevalent of late to the more or less exclusion of pneumonia and gastric disturbances.

The $B$. septus on the other hand appears to have lost much of its virulence; during the past twelve months its incidence has declined by nearly one half, and such cases as are due to it have been very mild and short.

TABLE IV.

Dr. C. H. Benham's Figures for Cases of Common Cold. Nov. 1905-Jan. 1906. 1907-08. I908-09. 1905-09.

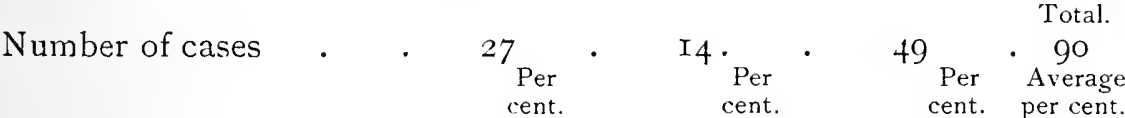

B. influenza . . . $3=\mathrm{II} \cdot 4=28 \cdot 6=\mathrm{I} 5 \cdot \mathrm{I} 6$

Pneumococcus . . (?) $=(?) \quad \cdot \quad 2=\mathrm{I} 4 \cdot 2=5 \cdot 5$

Streptococcus . . . $6=22,4=28 \quad$. II $=27 \quad \cdot 26$

M. catarrhalis . . . I $3=48$. $\mathrm{I} 3=93 \cdot 2 \mathrm{I}=52 \cdot 5^{8}$

$M$. paratetragenus . $.3=\mathrm{II} \quad . \quad \mathrm{I}_{4}=\mathrm{I00} \cdot 25=62 \quad \cdot 52$

B. septus . . . . $24=88 \quad$. II $=78 \quad \cdot 25=62 \quad \cdot 74$

Bacillus of Friedlander . I $=4$ (?) $3=2 \mathrm{I} \quad . \quad 5=\mathrm{I} 2$. II

(possibly more)

Dr. Benham and myself have compared our results from time to time, and such discrepancies as exist in our percentage figures for 1905-09 were largely due to the fact that the pneumococcus-influenzal epidemic at the very end of 1908 and beginning of 1909 appeared to reach London before Brighton, whereas the reverse was the case in the instance of the $M$. paratetragemus, which also failed to gain the same foothold in London that it did in Brighton; these peculiarities we noted at the time.

\section{TABLE V.}

Dr. Will Walter's Figures for Igo8-og in Ioo Cases of Rhinitis (Journ. Amer. Med. Assoc., September 24th, I9Io, p. IogI-Iog6).

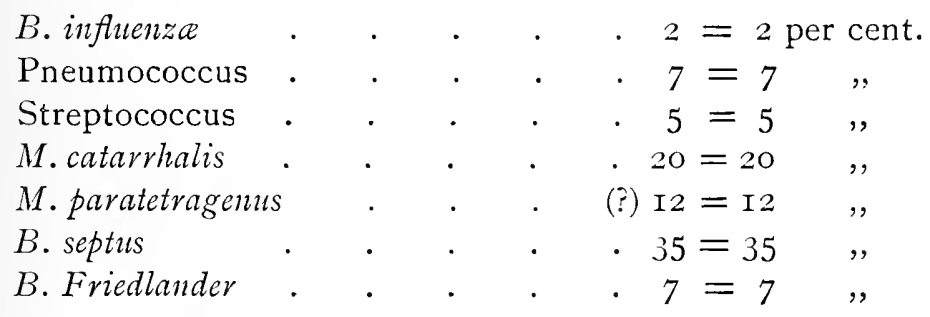

Swabs were taken from the nasal mucosa alone and cultures made on Loeffler's blood-serum, a medium which Walter subsequently recog- 
nised as not altogether suitable for the purpose; the results, therefore; are not strictly comparable with those of Benham and myself.

TAble VI.

C. E. West's Figures for 50 Cases of Chronic Post-nasal Catarrh (Proc. Roy. Soc. Med., February, I9I I, Otological Section, p. 43).

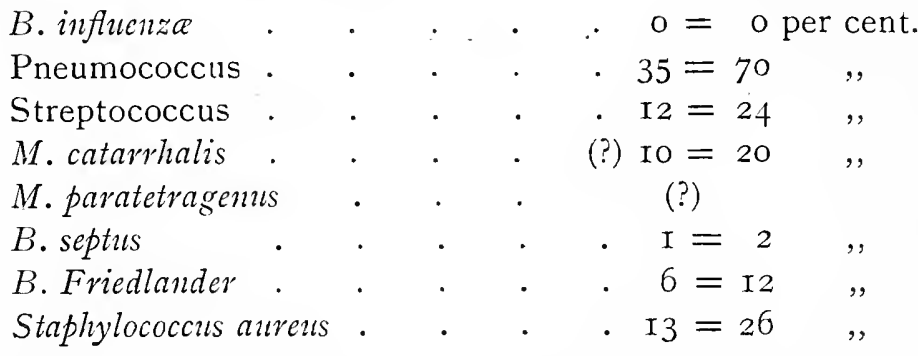

The period of time over which these investigations were made is not mentioned. Swabs were taken from the pharyngeal vault by means of a guarded swab; direct observations from smears of the swabs do not appear to have been made, and cases of acute nasal catarrh were carefully excluded, so that these findings are not to be compared with those of the previous observers. The chief points to be noticed are:

(r) The high percentage figure for the pneumococcus, viz. 70 per cent., which very closely corresponds to my own, viz. 66 per cent. for: all cases of nasal and post-nasal catarrh, acute and chronic, during the three years r $909-\mathrm{II}$.

(2) The very low figure for the Bacillus septus, which also coincides with my own observation of recent months. I am therefore inclined to view West's figures merely as representing the bacteriological conditions in post-nasal catarrh during a limited period of time and as corresponding to the incidence of pneumococcal infection of the respiratory tract generally.

(3) The complete absence of the B. influenza I can only attribute to the omission of observations upon direct smears and to a possible insufficient incubation period for the culture plates. During the past twelve months the B. influenza has been very slow of development.

(4) The high percentage incidence of the Staphylococcus aureus. West considers it a frequent factor in the production of chronic post-nasal catarrh and I am inclined to agree that it sometimes is.

To summarise, it would appear that any of the seven organisms, $B$. influcnza, pneumococcus, streptococcus, $M$. catarrhalis, M. paratetragenus, $B$. septus, and bacillus of Friedlander, alone or in varying combinations, may be responsible for a catarrhal condition of the upper respiratory passages. In perhaps 40 per cent. of cases one organisin so 
predominates as to justify the conclusion that it is the cause of the attack; more often two or more organisms are associated together, the $B$. influenze with the pneumococcus or $M$. paratetragenus, the $B$. septus with the $M$. catarrhalis or $M$. paratetragenus, so that it becomes very difficult to decide which organism or organisms stand in a directly' causal relationship to the attack. My own belief is that mixed infections from the beginning are fairly common.

In uncomplicated purulent nasal catarrh the streptococcus is the most frequent cause, next to it the Staphylococcus aureus. When sinus complications co-exist the B. influenza and pneumococcus are by far the most frequent bacteria concerned.

\section{(2) In Diseases of the Accessory Sinuses.}

The observations of Torne (Central f. Bakt. u. Parasit., Jena, I903, vol. iii, pp. 250-255) upon 36 cadavera in which the sinuses were found to be healthy would seem to indicate that in health these parts are remarkably free from bacteria; thus in 22 instances which were examined within two and a half hours of death the sterility was complete; of the remaining $\mathrm{I}_{4}$, which were examined between three and twenty-four hours after death, 7 were sterile, 7 contained a few bacteria. He also examined 26 cavities in 26 cadavera; 3 of these which were acutely inflamed were examined within one and a quarter hours of death, and in all the pneumococcus was present, in I case along with the Staphylococcus aureus. In 12 cases which showed a chronic purulent condition the bacteriology was most varied, streptococcus. Staphylococcus aureus, pseudo-catarrhalis, spirilla, protens, B. coli, and Friedlander's bacillus being among those found. Of the II cavities which showed slight catarrhal changes upon examination within three hours of death, 9 were sterile, I contained Staphylococcus aureus, I Bacterium sputigenum.

Herzfeld and Hermann (Arch. fur Laryngol. u. Rhinol., Berlin, I895) described the bacteriology of Io cases of antral suppuration examined during life; streptococci were present in 8, staphylococci in 7 , in both cases always along with other organisms, $B$. proten in $2, B$. coli in 3 ; one only was monorganismal, and that was due to the bacillus of Friedlander. Whether these observers made direct examinations from smear as well as from culture films I do not know, and it is also to be noted that at this time the B. influenzer was not as yet a well-known organism and may have been missed.

The results of Lewis and Logan Turner (loc. cit.) are very interesting. They examined 57 cases of antral disease, no other sinuses being involved, I of ethmoidal sinusitis, 4 of frontal sinusitis, and I2 antral cases complicated by involvement of the ethmoidal and frontal cells. 
The results in the 57 uncomplicated antral cases were as follows:

TABle VII.

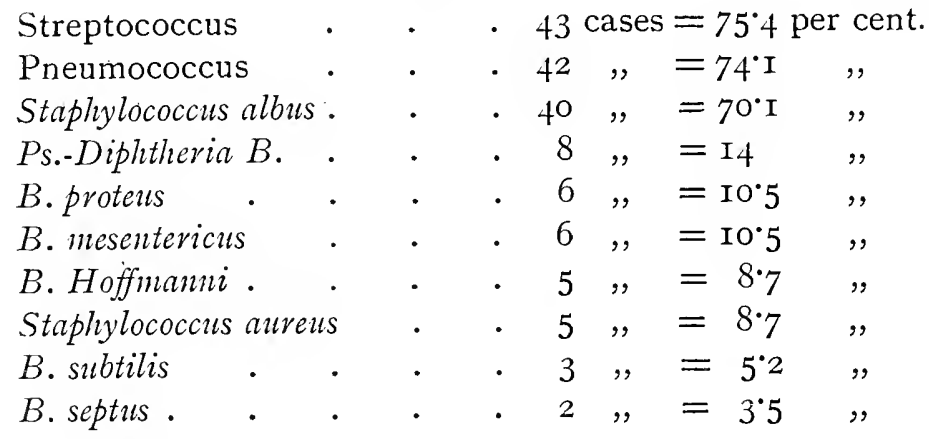

They also took swabs from 27 cavities during operation, and swabs from the nose after posturing in 42 instances.

The comparative results are as follows:

\section{TABLE VIII.}

\author{
Direct from cavity.
} cases.

$\begin{array}{lcccccc}\text { Pneumococcus } & . & 2 \mathrm{I}=77^{\circ} 7 \text { per cent. } & \text {. } 29=70 \text { per cent. } \\ \text { Staphylococcus } & . & 2 \mathrm{I}=77^{\circ} 7 & , & . & 28=66.6 & , \\ \text { Streptococcus } & . & 2 \mathrm{I}=77^{\circ} 7 & , & . & 27=64^{\circ} 3 & , \\ \text { Ps.-Diphtheria B. } & . & - & , & . & 8=\mathrm{I} 9 & , \\ \text { Hoffmann's B. } & . & - & , & . & 5=\mathrm{II} & ,\end{array}$

B. pyocyaneus, B. xerosis, B. perfringens, leptothrix, spirillum, and $B$. plexiformis were each found in one instance in swabs taken direct from the antrum, while $B$. influenze, B. Friedlander, B. coli, and $B$. buccalis maximus were each found only in swabs from the nose; these usually showed a more profuse flora than did those taken direct at operation.

In cases of less than one year's duration staphylococcus and pneumococcus were present in $84^{\circ} 6$ per cent., and streptococcus in $6 \mathrm{r} \cdot 6$ per cent., whereas in more chronic cases streptococcus was present in 80 per cent., pneumococcus in 70 per cent., and staphylococcus in 66 per cent. The almost total absence of the $B$. influenwa (one case only) is to be accounted for by the probable absence of any influenza epidemic during the time of their observations or within a year or two prior to them.

The one ethmoidal case was a chronic one and showed Streptococcus pyogenes and Staphylococcus aureus.

In the 3 cases of frontal sinusitis the findings were pneumococcus and Staphylococcus cereus flavus in one, staphylococcus, streptococcus and 
B. coli in the second, pneumococcus, Staphylococcus albus and aureus and Streptococcus brevis in the third.

From an experience of 30 cases of sinusitis examined during the years I909-IO-II I am unable to agree with Lewis and Logan Turner's figures, my results having been as follows:

TABLE IX.

\begin{tabular}{|c|c|c|c|c|c|}
\hline r of cases & . & $\begin{array}{l}1909 . \\
\text { IO }\end{array}$ & $\begin{array}{c}\text { I910. } \\
9\end{array}$ & $\begin{array}{l}\text { IgI I. } \\
\text { II. }\end{array}$ & $\begin{array}{c}\text { Total. } \\
30\end{array}$ \\
\hline & & & & & \\
\hline$a c i$ & . & $7=70$ & $=67$ & $9=8$ & $=73$ \\
\hline & & $5=50$ & $=67$ & $3=27$ & $=47$ \\
\hline & . & $=20$ & $=33$ & $5=$ & $=33$ \\
\hline & . & $t=40$ & $7=78$ & $4=36$ & $=50$ \\
\hline & . & 一 & $I=I I$ & $I=9$ & $=7$ \\
\hline & & - & - & . $\quad I=9$ & $=3$ \\
\hline & & - & - & . $\quad I=9$ & $=7$ \\
\hline & & $3=30$ & $=\mathrm{II}$ & $\cdot 2=\mathrm{I} 8$ & $=20$ \\
\hline 3. coli & & - & - & . $\quad I=9$ & $=3$ \\
\hline
\end{tabular}

In most instances examinations were conducted upon secretion obtained from the nose after posturing; in a few instances, however, in which the material was also obtained either at operation or through an old operation aperture, the results differed little in the two cases; the nasal secretion tending, as Lewis and Logan Turner pointed out, to present a slightly more complex flora. All the cases were chronic ones.

One of the cases was a very interesting one of ethmoidal sinusitis and greatly distended ethmoidal bulla; it was seen in conjunction with Mr. Herbert Tilley. The predominant organism was the B. influenze; streptococci were also present, and a very few $M$. catarrhalis.

If comparison of these results be made with the findings set out in Table III for nasal and post-nasal catarrh during the same period a general correspondence will be seen; this would probably be more marked if cases of post-nasal infection were excluded from Table III. It is, however, to be noted that nasal infections by the $B$. influenza seem especially prone to extend their energies in the direction of the accessory sinuses. The high incidence of the Micrococcus catarralis is almost certainly to be attributed to nasal swabs having been employed in the majority of my cases ; their numbers relative to the $B$. influenza, pneumococcus or streptococcus in any given case were, as a rule, quite low.

What I have already emphasised in connection with the statisţics for nasal catarrh I again emphasise here: Percentage figures have little 
value unless correlated over a long term of years; they are entirely dependent upon what organism or organisms exhibit special virulence during the period of observation. Again, there is no à priori reason why the infecting agent should not change from time to time. The $B$. influenza, for instance, may lose its virulence and even the power of maintaining a saprophytic existence, and be replaced by a pneumococcus which is then exhibiting particularly pathogenic properties, this latter, in its turn, being supplanted by a more hardy streptococcus; such a series of changes, in one or two instances, I have had an opportunity of observing.

Finally, in multiple sinusitis it must be remembered that the bacteriology of one infected cavity may differ totally from that of another even adjacent one. Since then the secretion obtained on one occasion may come from, say, a frontal cell, and that obtained on another occasion from an antrum or ethmoidal cell, this must not be taken as necessarily meaning a change of infecting agent in the event of dissimilar findings; this is a pitfall which one must carefully avoid.

\section{(3) In Eustachian Catarrh and Otitis Media.}

The recognition of Eustachian catarrh as a frequent cause of preventable deafness is becoming increasingly clear; its genesis is usually in a precedent nasal or post-nasal catarrh. Forcible efforts at expulsion of the nasal mucus during the stage of thickened secretion result in dilatation of the Eustachian tubes, and occasionally in the driving of some infected material into their orifices. This danger is greatly increased by the use of nasal douches-a practice highly commendable in itself, but one necessitating considerable care. Every patient when ordered to use a nasal douche should be warned of this, and told to allow the douche fluid to run away, and not to use any forcible efforts at expulsion of it with its contained bacteria and dissolved mucus until the cavities have been thoroughly washed out. Another hint I have found to have decided value: by firmly pressing the tragus of the ear with a fingertip into the external meatus, counter-pressure is extended through the tympanum into the middle ear and Eustachian tube, and the risk of dilatation considerably reduced. I have noted that with most people one tube as a rule dilates much more readily than the other, and that if counter-pressure be applied to the side more liable to dilatation the douche fluid and mucus may then be forcibly expelled without danger to the Eustachian tube.

This, then, being the way in which Eustachian catarrh is perhaps commonly set up, it follows that the bacteriology of Eustachian catarrh 
should correspond to that of nasal and, more especially, post-nasal catarrh. Unfortunately, reliable observations are lacking owing to the almost insuperable difficulties of obtaining swabs free from contamination with nasal or pharyngeal mucus. The few observations upon which any reliance could be placed that I have been enabled to make, by the kindness of Dr. Greville Macdonald, Mr. G. S. Hett, and others, have tended to show that the $M$. catarrhalis, $B$. influenza, pneumococcus, and streptococcus are the bacteria which most commonly set up catarrh of the Eustachian tube; upon one occasion B. septus was obtained in pure culture from a swab taken from the orifice. Clinical observation would tend to confirm these results, as the colds due to these four organisms are more particularly the ones in which signs of involvement of the Eustachian tubes are most evidenced.

That many, if not most, cases of otitis media are sequent to Eustachian catarrh is probable. In scarlet fever and measles the streptococci almost certainly find their way to the middle ear vi $\hat{a}$ the Eustachian tube. This, however, is not the only route; typhoidal otitis media, for instance, probably arises from the blood infection, and the same may be true of some pneumococcal and influenzal cases. The $B$. coli group, B. proteus group, and staphylococci also give rise to this condition, while otitis media due to the $B$. pyocyanens, spirochætes and streptothrix has been reported; as these organisms are fairly common inhabitants of the mouth, infection via the tube is probable in these cases.

\section{(4) In Tracheitis and Laryngitis.}

Here, again, reliable bacteriological observations are lacking, but the probable truth can be deduced from clinical observations in the following way. There are certain definite clinical types of catarrhs which at one time or another in their course involve the larynx and trachea. The bacteriological findings remaining more or less constant throughout the attack, the inference appears to be justified that the bacteria which are responsible for the nasal, post-nasal, bronchial and pulmonary catarrh are likewise responsible for the tracheitis and laryngitis. For instance, we have the three following types of catarrh: (I) Definitely located in the larynx there is a dry, rough feeling as if sand-paper had been employed to line the space, the rough surface towards the mucous membrane. This gives rise to a dry, hard cough, which in a day or two may become paroxysmal ; after a severe fit of coughing a tiny pellet of mucus, clear and very viscid, may be expelled. The infection extends upwards and downwards simultaneously, so that at the end of three to five days the bronchi are definitely involved, mucous râles and rhonchi are to be heard in the chest, while nasal and post-nasal discharge is 
abundant. The mucus is no longer clear and viscid, but yellow, mucopurulent, tending to be nummular, and easily spread upon a slide. This is a description of a type of cold during the course of which practically nothing but pneumococcus is to be seen in the secretions, whether this be the tiny pellet expelled from the larynx during the first day or two or in the later muco-purulent discharge. The pneumococcus may therefore be assumed to have been responsible for the initial laryngitis. (2) A second type begins in a very similar manner and may likewise extend down into the chest and up into the nose; the secretion, however, remains clear and viscid throughout, and in it the $M$. paratetragenus, sometimes in pure culture, sometimes along with the $M$. catarrlalis or pneumococcus, is to be found. The $M$. paratetragenus would therefore appear to cause a laryngitis. (3) Again, a dry, harsh feeling of the soft palate and post-nasal space may be followed in twenty-four to thirty-six hours by an acute rhinitis; the infection then spreads steadily down the larynx and trachea into the large bronchi. Cough is marked, but rarely paroxysmal, and copious, thin, clear mucus, only in the later stages streaked here and there with muco-purulent strands, is easily voided. This type of cold appears to be due to the $M$. catarrhalis, but as other bacteria are commonly present in the secretions from the beginning it is with less certainty that one can attribute the laryngitis and tracheitis to the $M$. catarrhalis alone; however, such I believe to be the case.

Similarly, the B. influenze, and with less certainty the streptococcus, appear to be capable of setting up this condition. On the other hand, I have never found these parts involved in catarrhal conditions due to the $B$. septus or bacillus of Friedlander. As regards the latter, however, inasmuch as at times it does attack the chest, it is highly probable that it is capable of acclimatising itself in the trachea and larynx. I can only say that with an experience of eighteen catarrhal attacks due to the Bacillus of Friedlander I have never seen these parts involved.

Roughly, in order of importance, I should arrange the causes of acute catarrhal laryngitis and tracheitis as follows: Pneumococcus, $B$. influenze, $M$. catarrhalis, $M$. paratetragenus. It is hardly necessary for me to emphasise the fact that I am here dealing only with purely catarrhal conditions; the diphtheria bacillus is, of course, the most important invader by far of the larynx and trachea, next to it the tubercle bacillus.

(5) In Pulnonary Catarrh, Bronchitis, and Asthma.

The deeper down the respiratory tract the source from which the secretion is voided, the greater obviously the danger of contaminating 
bacteria being picked up on the way; the uncleanliness, from the bacteriological standpoint, of the fauces and mouth I have already alluded to, and it would, at first sight, appear to be almost impossible to conduct reliable observations upon material coming from the bronchi, bronchioles, or pulmonary cells. Such, however, is not the case. The precautions outlined on pp. 9 and ro will reduce the adventitious microbes to a minimum, and a knowledge of the cytology of the sputum, as outlined on pp. 13-15, will enable us to decide whether the actual sample under examination has been voided from the desired locality. In Table $\mathrm{X}$ are set out the results of my examinations in pulmonary catarrh and bronchitis during the three years I909-II.

TABle $X$.

\begin{tabular}{|c|c|c|c|c|c|c|}
\hline \multirow{2}{*}{ Number of cases } & \multicolumn{2}{|r|}{$\begin{array}{l}\text { Ig09. } \\
5 \mathrm{I}\end{array}$} & \multicolumn{2}{|l|}{$\begin{array}{c}1910 . \\
33\end{array}$} & $\begin{array}{l}1911 . \\
20\end{array}$ & \multirow{2}{*}{$\begin{array}{c}\text { Total. } \\
\text { Iot } \\
\text { Mean } \\
\text { percentage }\end{array}$} \\
\hline & & $\begin{array}{c}\text { Per } \\
\text { cent. }\end{array}$ & & $\begin{array}{l}\text { Per } \\
\text { cent. }\end{array}$ & $\begin{array}{l}\text { Per } \\
\text { cent. }\end{array}$ & \\
\hline B. influenza & & $2 I=42$ & $9=2$ & 27 & $\mathrm{I} 2=60$ & 40 \\
\hline Pneumococcus. & & $22=44$ & $I 8=5$ & 54 & $I_{4}=70$ & 52 \\
\hline Streptococcus & & $2 I=42$ & $20=6$ & 60 & $\mathrm{I}_{4}=70$ & 53 \\
\hline M. catarrhalis . & & $3^{6}=7^{2}$ & - $23=6$ & 69 & $16=80$ & 72 \\
\hline$M \cdot$ paratetragenus & & $\mathrm{I} 2=24$ & $9=2$ & 27 & $3=15$ & 23 \\
\hline Bacillus of Friedla & & & & & & \\
\hline group & & $5=\mathrm{IO}$ & $\mathrm{I}=$ & 3 & $I=5$ & 7 \\
\hline B. septus & . & $I=2$ & - & & $I=5$ & 2 \\
\hline Streptothrix & . & - & 一 & & $3=I_{5}$ & 3 \\
\hline
\end{tabular}

If comparison of these results be made with those for the same years for nasal catarrh set out in Table III, the only point of material difference will be seen to be in what I have already referred to-the great disinclination of the $B$. septus to make its habitat in the lower respiratory passages. These figures must also be taken as representative only of the infecting agents during the years Igog-II ; should any given organism lose its virulence, naturally it will cease more or less to appear as an infective agent in the lower respiratory tract, just as it will do in regard to the upper passages.

One other point is deserving of attention, namely this, that infections of the lower tract are commonly mixed ones, more commonly so, perhaps, than are those of the upper; the reason of this I take to be the fact that infections of the lower passages frequently follow on those of the upper, and that these latter, as the attack progresses, tend to. become more and more mixed infections. The Micrococcus catarrhalis alone, for instance, certainly is able to initiate and maintain an acute bronchial infection, but more commonly it appears as a secondary infection to the 
pneumococcus or $B$. influenza. Pure influenzal infections, again, are relatively uncommon; more often they are complicated by the pneumococcus or streptococcus.

The importance of the pneumococcus as the causative agent for certain cases of acute suffocative catarrh has been well brought out by Samuel West (Proc. Roy. Soc. Med., April, IgI I, Med. Sect., p. Ior), and I am inclined to regard this organism as at the same time the most frequent cause of bronchial catarrh and the most dangerous to the patient. It is also worthy of notice that the $B$. infuenza may be resident in the bronchi or pulmonary cells, giving rise to marked constitutional, but few localised, symptoms; indeed the sputum voided may be almost nil.

The findings in asthma.-As will be quite apparent when I come to consider the vaccine therapy of asthmatic conditions I am very far from considering bacteria as the universal cause of asthma, but regard them merely as one of the excitants of the asthmatic attack, as one of the agents capable of stimulating the nerve-endings and unstable centre. Where bronchitic symptoms àre prominent the importance of bacteria is correspondingly greater. During the years I909-II I investigated the flora of the sputum collected with suitable precautions a few hours after the commencement of the attack in fifty-one cases. The results are set out in Table XI.

\section{TABLE XI.}

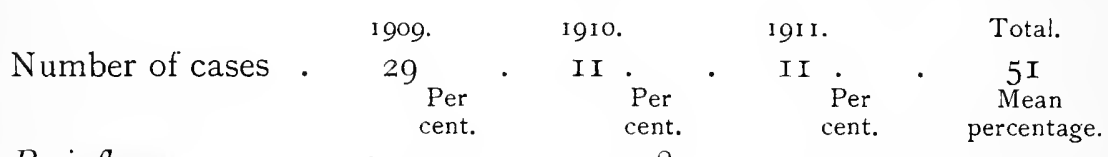

B. infuenza. . $3=\mathrm{IO} .2=\mathrm{I} 8 \cdot \mathrm{I}=9 \cdot=\mathrm{I} 2$

Pneumococcus $\quad 7=23 \cdot 4=36 \cdot 2=18 \cdot=26$

Streptococcus $\quad \cdot 26=90 \quad \cdot$ II $=$ IOO $\cdot$ II $=$ IOO $\quad=96$

M. catarrhalis . I $8=62.8=73 \cdot \mathrm{IO}=9 \mathrm{I} \cdot=72$

$M$. paratetragenus . $4=\mathrm{I} 3 \cdot 3=27 \cdot 3=27 \cdot=20$

Comparison of these results with those set out in Tables III and X brings out several points of interest. The low percentage incidence of the $B$. infuenze and pneumococcus is very marked, but when they do occur their significance I believe to be correspondingly greater. The almost universal occurrence of the streptococcus group is most striking, and corresponds, I believe, to its ætiological importance. The two chief varieties of streptococcus found have been already described on pp. 28-30. The incidence of the $M$. catarrhalis and $M$. paratetragenus is approximately the same in all three tables, and their importance in asthma is very difficult to determine. The organism described 
by Carmalt-Jones as a possible cause of asthma he now tells me he is inclined to regard as belonging to the $M$. catarrhalis group.

One thing which will certainly strike the investigator into the bacteriology of respiratory affections is the much less complexity of the findings in the asthmatic sputum as compared with that derived from other catarrhal affections. The total number of bacteria present is not only, as a rule, much less, but monorganismal and binorganismal specimens are much more frequently encountered.

\section{(6) In Whooping-Cough.}

Lack of material has prevented me making any extended investigations of my own upon this question. The very careful and laborious investigations of Freeman at St. Mary's Hospital and of Martha Wollenstein in America have fortunately rendered any further work almost quite unnecessary. These observers corroborate the work of others, and regard the establishment of the bacillus of Bordet-Gengou as the specific cause of whooping-cough as quite complete. They have, however, in addition brought out this important point : infection is very rarely a pure one; simultaneous or secondary infection by the B. influenza exists in a very large percentage of cases (nearly go per cent.), while the same holds true of the pneumococcus to a considerably smaller yet important degree. This accounts for the modified success which has attended the treatment of whooping-cough by means of a vaccine of Bordet's bacillus alone, and will be more fully referred to subsequently.

\section{(7) In Pulmonary Phthisis.}

In this, the most important of all infections of the respiratory tract, accuracy in the diagnosis of the organisms, which accompany the tubercle bacillus, becomes more than ever necessary. Fortunately the secretion from a lung affected by phthisis by its very nature is much less likely to pick up contamination as it is voided than is the sputum of other infections. Provided the precautions detailed for the collection of specimens on pages 9 and ro are carefully followed, the nummular masses of sputum only require washing twice or thrice in sterile salt solution to free them from adventitious microbes; or instead of washing, a suitable sample may be placed in a well-heated platinum dish for a few seconds; it is then turned over and the other side likewise cooked; by means of a sterile knife the mass may then be incised and specimens taken for direct and cultural examinations from the cold interior. Of course, when the sputum is not nummular the preliminary precautions must be carried out with especial care. Personally, I direct my 
patients to make but one expectoration into a bottle, and prefer the early morning specimen; at the same time it is well to take two or three other specimens at intervals during the day, each in a separate bottle, for it by no means follows that the secondary infection at one focus of tuberculous disease will correspond to that at a second or third. How the importance of such organisms as are isolated in the diseased processes can be determined by laboratory and by clinical tests I shall detail later. Observations upon the bacteria concerned in conditions other than that of pulmonary phthisis consumed so much of the eight years' period which I have devoted to the study of the bacterial diseases of the respiratory tract that it is only during the last three years that I have been able to study the mixed infections of phthisis with adequate care. The results of my observations are set out in Table XII.

\section{TABLE XII.}

The Mixed Infections of Pulmonary Phthisis.

$\begin{array}{cccccc} & \text { 1909. } & \text { 19ro. } & \text { I9II. } & \text { Total. } \\ \text { Number of cases } & \text { I4 } & \text { I2. } & \text { I2 } & \text { I6 } & 42 \\ \text { Per } & \text { Per } & \text { Per } & \text { Mean } \\ \text { cent. } & \text { cent. } & \text { cent. } & \text { percentage. }\end{array}$

\begin{tabular}{|c|c|c|c|c|c|c|c|c|}
\hline B. influens & & . & $I=$ & & - & $6=$ & & \\
\hline Pneumoco & ccus & . & $3=2$ & & $5=42$ & $7=43$ & & \\
\hline reptoco & cus & . & $\mathrm{II}=7$ & & $9=75$ & $\cdot I_{4}=87$ & & \\
\hline catarrh & alis & . & . $\mathrm{I} 2=8$ & & . IO $=83$ & . $\mathrm{IO}=62$ & & \\
\hline$M$. paratet & ragenut & & $5=3$ & & . $4=33$ & I & & \\
\hline B. septus & & & $\mathrm{I}=$ & 7 & - & . & & \\
\hline Bacillus o & Fried & llander & $\mathrm{r} \quad \mathrm{I}=$ & 7 & - & . $\quad I=6$ & & \\
\hline S. albus & - & . & - $\quad \mathrm{I}=$ & 7 & $4=33$ & . $2=$ & & \\
\hline S. aureus & . & . & - & & . $.2=16$ & . $3=1$ & & \\
\hline Proteus & & & $\mathrm{I}=$ & 7 & - & . $\quad I=6$ & & \\
\hline B. coli & & & - & & - & - & & \\
\hline
\end{tabular}

The yearly variations from the mean are by no means inconsiderable in the instance of some of the bacteria, the B. influenza, pneumococcus, and $M$.paratetragenus, for example; this, as before, may be due to a periodic rise and fall in the pathogenicity of these micro-organisms. One point which I feel is worthy of attention is this: there is a great tendency to speak of these as secondary infections; the term mixed infection is far preferable, for such evidence as we have supports the view that in many instances the pneumococcus, $B$. influenza or streptococcus is the primary infection, that by the tubercle bacillus the secondary one. 
The small percentage of cases in which I have found the staphylococcus to be concerned is somewhat striking, the percentages of cases also in which I have found the $B$. influenze and pneumococcus present are also considerably lower than in the cases of pulmonary catarrh and bronchitis for the same years (vide Table X), but correspond much more closely with the figures for asthma (vide Table XI), as does also the percentage figure for the streptococcus. 


\section{CHAPTER V.}

\section{THE VACCINE THERAPY OF RESPIRATORY DIS- ORDERS.}

IV (a) General considerations.-As vaccine treatment is essentially nothing more nor less than an attempt at the artificial stimulation of such means of defence against bacterial invasion as the tissues of the body already possess when these threaten to make default, it is obviously necessary first to consider what are the defensive mechanisms of the body against the bacterial invasion of the parts now under consideration.

As we have already seen, nature has endowed man with a bacterial filtration apparatus at the very vestibule of the true respiratory tract.

The nasal vibrissæ and ciliated epithelial cells with their coating of sticky mucus act as a filter of quite extraordinary efficiency. Some years ago I endeavoured to estimate the actual number of bacteria which escaped involvement in the nasal passages in the case of purely nasal respiration of London air. Accurate observations proved very difficult to perform, and I obtained too few thoroughly satisfactory ones to make them worthy of publication. The conclusion, however, appeared to be justified that at least 95 per cent. of the organisms entering the nose were entrapped therein, and accordingly failed to appear in the respiratory air as it entered the larynx; of the remaining 5 per cent. or less by far the greater percentage must be caught up by the ciliated epithelium of the larynx and trachea; still, as we have seen, a certain number do undoubtedly enter the bronchi and reach the lung.

What, then, is the fate of these entangled bacteria? The statement has been made that the respiratory mucus possesses bactericidal properties. This I have proved beyond question to be wrong; that it may be inhibitory of bacterial growth to a slight extent is possible, but this my own experiments fail entirely to confirm.

It would thus appear that the function of the mucus is a purely 
mechanical one; in health when it is secreted in small amount it serves merely to entangle the bacteria and prevent their access to the pulmonary tissues; in pathological conditions it is increased in amount, and serves in addition to wash the multiplying bacteria and their toxic products away from the inflamed areas. Such bacteria as succeed in penetrating this defence have now to meet the opposition of the epithelial cells. No cells of the body would appear to be possessed of more strongly phagocytic powers than these epithelial cells of the upper respiratory tract; both in the healthy and pathological state they are always to be seen crowded with bacteria. How far their phagocytic power is independent of the co-operation of opsonins and other immune bodies has not been determined, but that their inherent phagocytic power is very considerable is beyond question.

As a rule these defensive agencies suffice to protect the upper respiratory passages against bacterial attacks; at times, however, the virulence or number of the micro-organisms may be such that the outworks are penetrated and the inner lines of defence attacked. The lymphoid cells and polynuclear leucocytes now have their parts to play, and herein probably receive considerable assistance from the immune bodies of the tissues. Should these prove incapable of localising the conflict systemic infection may result, as is sometimes seen in the case of the B. influenza, B. tuberculosis and pneumococcus, and the lymph-nodes, liver, spleen, and pulmonary tissues are involved in the struggle, filtering off and destroying the invaders with the assistance of the opsonins, lysins, agglutinins, and other immune bodies. Granting the truth of these views it becomes at once apparent that inasmuch as "immune bodies" play but a subordinate rôle in protecting the respiratory passages against bacterial invasion, if we rest content merely with the endeavour to stimulate the over-production of "immune bodies" by the artificial introduction into healthy tissues of the corresponding bacterial vaccines, we shall be failing in our full duty to the patient. Let us then consider what are the essential preliminaries to any scheme of vaccine treatment.

First and foremost, it is little use that nature has endowed man with an efficient bacterial filter if from various causes he fail to avail himself of it. Nasal respiration is a habit which should be taught in the cradle, and far too seldom is. No nurse or mother should allow an infant to sleep with the mouth open; gently closing it when the child is asleep will usually suffice; should this not be the case, then an elastic band may be fixed gently over the point of the chin in order to secure the desired result; this will have the additional good effect of preventing the child sucking its thumb, and so distorting the palatal arch. The due observance of these precautions will do much to obviate 
the subsequent appearance of enlarged tonsils and uvula, and of adenoid growths.

When these occur in children who do not perform nasal respiration careful training in the habit will frequently suffice to cause their disappearance ; should this, however, not result, or should nasal respiration prove impossible from their presence, surgical measures must perforce be taken, and careful training be then begun.

In the event of the patient having already reached adolescence or mature life attention to this point becomes even yet more urgent. Deflections of the septım, enlarged turbinate bones, polypi, adenoids or enlarged tonsils may each render nasal respiration difficult, or even impossible, to carry out. In this case surgical measures should be insisted on, and their performance encouraged by assuring the patient that they alone will frequently suffice to rid him of all his troubles without recourse being had to a long and costly course of therapeutic immunisation.

When abnormalities have been adequately dealt with it still remains to teach the habit of nasal respiration. 'This procedure can be made not altogether unattractive to the patient in the following way. Note is made of the maximum power of expanding the chest, and he is given these instructions: A watch is to be placed by the bedside in such a position that it can readily be seen when the patient is lying in bed flat upon the back. A very low pillow, or none at all, is placed under the head, and the dorsal position assumed, legs straight and arms by the side, the only covering a single sheet. The lungs are now emptied as completely as possible, the residual air being got rid of by means of two or three forcible little expirations, and by bringing each arm firmly over the points of the opposite shoulder. The arms are now replaced by the side, and air taken in through the nose as slowly as possible; when the lungs are apparently full it will be found that a little more air can still be taken in by slightly arching the back and drawing back the shoulders. The time is now noted, and the breath held; after a certain interval the patient will feel that he can hold it no longer; as he becomes more and more practised he will find that in reality he can retain the air until long after the head begins to swim, but this may well be left until some proficiency has been attained. A glance is then given at the time, and forcible expiration performed as speedily as possible through the open mouth.

The whole procedure is then begun $a b$ initio and repeated only twice. This is done regularly night and morning. It is no uncommon experience of mine to find that a patient who at the beginning of a course could retain the breath for no more than forty-five seconds, will at the end of four to six weeks' practice retain it with even greater ease 
for quite two minutes, and that the chest expansion has meanwhile gone up from $\frac{3}{4}-\mathrm{I}$ in. to $2-2 \frac{1}{2}$ in. Some patients regard this training as an attractive competition with themselves. It then remains to urge that whenever they are in the country walking on the hills or heath, that they should remember several times to fill and empty their lungs completely.

In certain cases slight modifications have to be made; for instance, asthmatic cases should be advised that thorough expiration is even more important than thorough inspiration; emphysematous cases must be warned to conduct the exercises with due discretion, that, inasmuch as damage has already resulted to the pulmonary tissues, which are unduly thin and fragile, too forcible inspiratory and expiratory movements are to be avoided lest further damage be the result.

Not only will this training in correct respiratory methods render nasal respiration easier of performance until finally it becomes a habit, but improved nutrition of the tissues generally, and thereby increased power of elaborating immune bodies therein and so of resisting bacteriai attacks, will be brought about.

On p. 9 I have referred to the supreme importance of scrutinising the mouth thoroughly for the presence of pyorrhœa alveolaris and follicular tonsillitis. It is little use endeavouring to free a nasal cavity of streptococcus or Micrococcus catarralis by the injection of the corresponding vaccine when there are pockets round the teeth or in the tonsils filled with these bacteria, more or less inaccessible both to the action of immune bodies and phagocytic cells. Crowns and bridges in a mouth I look upon as an utter abomination, and have never yet seen the mouth containing them in anything but a highly septic state. The practice, too, of leaving in the mouth one or two teeth around whose margins pyorrhoea exists merely for the better fixation of a denture is again a proceeding which cannot be too strongly condemned. Even if the micro-organisms responsible for the pyorrhœa are in no way concerned in the respiratory condition, yet the toxins they elaborate are so potent that a general lowering of the resisting powers of all the body tissues is the inevitable result. As I have already said, such supreme importance do I now attach to the presence of pyorrhœa alveolaris in sufferers from respiratory disorders, that should a patient refuse first to have this condition adequately treated by a thoroughly competent dentist, then I firmly and finally refuse to undertake the treatment of the respiratory affection.

These preliminaries having been arranged, it now behoves us to consider in what other ways the defensive mechanisms of the body can be aided.

The mechanical removal of the bacteria by means of the mucus 
can be assisted by the careful use, night and morning, of the nasal douche; to the precautions necessary in its use I have already alluded on p. 50. The most suitable liquid by far of which I have made trial is glycothymoline I part, warm water 3-5 parts; not only is this quite unirritating and pleasant to use, but its solvent action on mucus is particularly good. Hygienic individuals are careful to bathe, to wash their hands and face, and clean their teeth, but the nasal cavities with the accumulated dust and dirt and bacteria of the day they are wont entirely to neglect. The careful use of the nasal douche night and morning is therefore much to be recommended. When the nasal mucosa is chronically inflamed and over-dry, as in atrophic rhinitis, the use of a mildly antiseptic but soothing oily spray in an efficient nebuliser is also advisable.

The following prescription may prove of use :

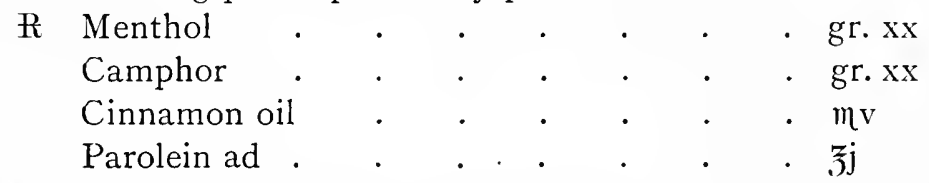

When follicular tonsillitis does not prove to be entirely amenable to surgical measures, careful painting of the parts twice or thrice daily with a solution of I part of chinosol in 200 to 300 parts of glycerine by means of a fine camel's hair brush may prove of service-for this hint I am indebted to my friend Mr. F. J. Steward-while sucking formamint lozenges also aids in reducing the bacterial content.

Where accumulations of pus are present, as in infections of the accessory sinuses, it is obviously necessary that these be removed either by lavage, as in acute sinusitis, or by a surgical operation to establish free drainage, as in chronic sinus infections which refuse to yield to lavage.

It is beyond the scope of this paper to go further into the treatment of the various local conditions which may complicate a more general respiratory disorder; it only remains to say that the careful attention to all abnormalities may determine the final success of any more general form of treatment as by means of vaccines.

Assuming, then, that due regard has been paid to all preliminaries, we may proceed to the consideration of vaccine treatment, its rationale and method of employment. Vaccine therapy depends upon this principle: "That the animal organism is capable of elaborating antibodies to any foreign albuminous substance introduced into it, which is soluble in the tissue fluids, and does so to an amount which is in excess of that required to neutralise the quantity of foreign matter introduced."

Now bacteria are foreign albuminous substances, and the question at once occurs-Why, if this principle be true, does the body not conform 
to it by manufacturing more than sufficient antibodies to the bacteria to neutralise them and so put an end to the infection? The answer to - this is that in the great majority of instances it does so do ; that when it fails several factors may conspire to hinder its good work, such as the following :

(I) The bacteria may be introduced in such numbers that the immunising machinery may be paralysed locally or generally.

(2) That when this paralysis is local other regions of the body which may be more than capable of making good the local defect in production of antibodies are incapacitated from so doing by lack of the necessary stimulus, viz. the bacteria are not present there to give the stimulus.

(3) A more than adequate supply of antibodies may be formed in the body generally, but may not reach the areas where they are required owing to coagulation of sero-albuminous exudate or formation of thickened walls around the infected foci.

(4) Certain strains of bacteria seem to be incapable of exciting the formation of immune bodies; this would seem to be the case especially when their virulence is low.

(5) The tissues infected may be ones incapable of forming immune bodies altogether or only in very small quantities; this may, perhaps, be the case with the superficial layers of the epidermis. It is hard to see how bacteria infecting the most superficial layer or layers of the skin, the sweat, sebaceous and mucous glands can excite the production of antibodies; toxins may perhaps be absorbed, and antitoxins elaborated, but unless the bacteria themselves penetrate the deeper layers, anti-bacterial bodies, such as opsonins, lysins, agglutinins, can hardly be formed. It will be urged that in such instances the bacteria are maintaining a merely saprophytic existence; this is more or less true, but none the less I would maintain that they may be provocative of conditions unpleasant to the host, such as the formation of excessive secretion in the nasal or urethral passages.

(6) There may be defective power, either congenital or acquired, on the part of the tissues generally in responding to bacterial invasion by the elaboration of antibodies. This I believe to be a more common phenomenon than is usually considered to be the case, and to afford explanation of some of the failures experienced in vaccine treatment.

Let us consider how these various obstacles to the establishment of immunity may be overcome.

(I) When the paralysis of the immunising machinery is general, the only hope would appear to lie in the administration, best locally into the infected areas, of sera, which should, if possible, possess both antitoxic and bactericidal properties. Unfortunately, ideal sera have 
not yet been found capable of production. Anti-diphtheritic serum is antitoxic but not bactericidal; anti-streptococcal serum appears to be bactericidal rather than antitoxic; anti-cholera serum is only lytic; the anti-meningococcic serum of Flexner appears to be both bacteriolytic and antitoxic, but is not yet an ideal serum.

(2) When the paralysis of the immunising machinery is purely local, the production of antibodies may be incited by the introduction into healthy tissues of the suitable corresponding vaccine.

(3) The supply of antibodies in the body generally being sufficient, local deficiency is to be obviated in the various ways which Sir Almroth Wright has so carefully indicated, viz. abscesses may be opened, the thickened walls scraped, and the abscess cavity packed with citrated salt solution, the citrate decalcifying and so preventing the coagulation of the lymph, the salt increasing osmosis through the infected tissues; the coagulability of the blood generally may be reduced by large doses of citric acid, hyperæmia of infected areas may be brought about by local applications of heat or by passive congestion (Bier's method).

(4) Diminished power of exciting antibody formation on the part of the infecting bacteria may sometimes be obviated by the introduction into the tissues of a heterologous vaccine of proved efficiency. Instances. of this are chronic endocardial infections by the gonococcus and $B$. influenza, some long-standing local infections by the gonococcus and possibly by the streptococcus.

(5) This class of infection is one to which local antiseptic treatment would appear to be applicable rather than vaccine therapy, and so, in fact, it usually is. At the same time I have seen instances in which careful and persistent local antiseptic treatment has failed yield well when vaccine treatment was combined with the former.

(6) remains one of the problems for future studies in immunity to solve; it is, I believe, only an exaggerated form of the failures on the part of the tissues to establish local immunity. My own feeling is that our present knowledge of immunity is fragmentary in the extreme, and that nothing that we know at present suffices to explain why one individual should throughout life be susceptible above his fellows to the attacks of the staphylococcus and another individual be apparently immune against the staphylococcus, but fall a ready victim to the bacillus of tuberculosis. The chemical composition of the tissues as determined probably by metabolic processes is much more intimately concerned in immunity than is at present realised. Just as one field will grow good wheat but not good potatoes, and another field good potatoes but bad wheat, so with the tissues of the human race; lysins, opsonins, agglutinins, and all the other known immune bodies are but 
barriers raised against bacteria, which are already present and in process of multiplication. True immunity, I feel sure, does not depend on these, but on some as yet quite unknown conditions which render the tissues unsuited to the very existence of, or rather unattackable by, an invader. To make my meaning clearer, our tissues are an island protected by a fleet; it is upon our fleet that we depend for our existence, not upon the land soldiers, which merely form a second line of defence to the fleet. Opsonins, lysins, and agglutinins are but land soldiers; of our fleet we as yet know nothing, and our imperfect efforts are perforce directed to the strengthening of our land forces.

\section{The Control of Dosage and Intervals.}

As our main consideration is the strengthening of the defences by the aid of therapeutic inoculations, it is obviously necessary to discuss the means whereby determination may be made of the appropriate times and degrees of the augmentation, or in other words of ascertaining appropriate intervals and dosages. That opsonic index determinations have taught most of what we know in regard to the conduct of therapeutic immunisation will be readily granted, and it is therefore with some hesitation that advocacy is made of other methods of control.

The bacterial diseases of the respiratory tract may be divided into two categories: (I) Those in which at some part of their course, usually at the beginning of the attack, the bacteria circulate in the bloodstream, viz. pulmonary tuberculosis, pneumonia, and sometimes, at all events, true influenza. (2) Those in which the bacteria throughout the course of the attack are localised more or less entirely in the epithelial and endothelial cells, and in the tissues in immediate juxtaposition to these; instances of this are infections of the nasal and pharyngeal mucosa by the $B$. septus, of the laryngeal and bronchial mucosa by the II. catarrhalis or Streptococcus maximus.

Constitutional symptoms, when present, must here be due to absorption of toxins, either excreted by the bacilli or the result of tissue degeneration. Such immunity curves as research has been able to evolve pursue more or less constant courses in both classes of infection, and are determined by (I) the responsive powers of the individual to an immunising stimulus, and (2) the force of the immunising stimulus. The observatious by G. G. MacDonald (vide Studies in Pathology, edited by W. Bullock); by Eyre (Vaccines and Sera), and by Giglioli and Stradotti (Interno alle Modificazione dell' indice opsonico nel corso di alcune malattie acuta da infezione), who especially studied and correlated the temperature and opsonic index curves in various cases of pneumonia and other acute infections 
show that in practically all instances at the beginning of infection the opsonic index is low $\left(0^{\circ} 4^{-0} 8\right)$ and temperature considerably above normal. Should the disease run a favourable course it is found that at the onset of resolution there is a sudden and abrupt rise in the opsonic index and in the number of leucocytes and a corresponding fall in temperature (see Chart I), whereas in those cases which run a fatal course the opsonic index is persistently low, and the temperature continues pyrexial in type until near the end.

In the former class of case the tissues have succeeded in elaborating an adequate supply of antibodies, antagonistic to the pneumococci and

CHART I.

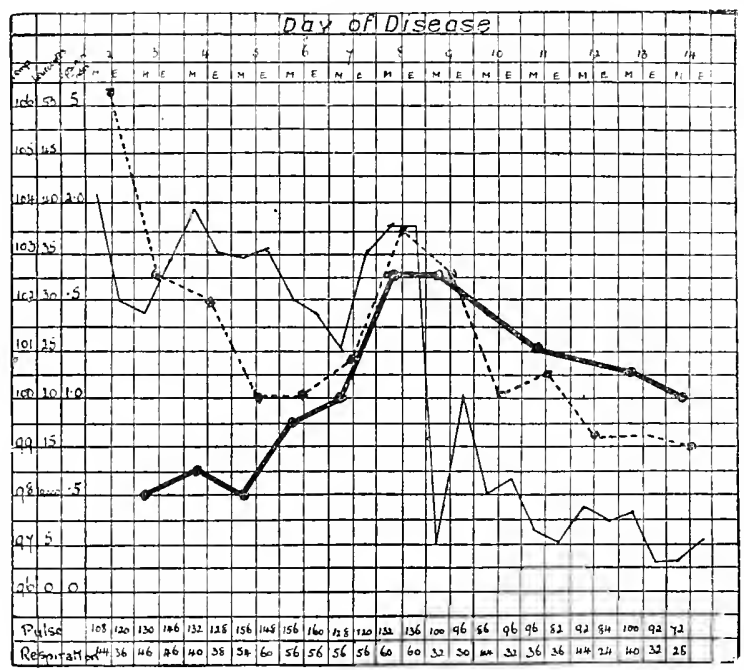

Relation between Leucocytes, Opsonic Index and Temperature in a Case of PNeumonia (Eyre).

Dotted line $=$ number of leucocytes per cubic millimetre; thick line $=$ opsonic index ; thin line $=$ temperature.

their products; in the latter class of case the tissues are unable to respond to the stimulus, either from an intrinsic defect or from the fact that the stimulus was excessive and paralysing in its effect, and the supply of antibodies is therefore inadequate to cope with the invaders and their toxins. In the localised infections, such as nasal and bronchial catarrh set up by the B. septus and M. catarrhalis respectively, Benham and myself have observed that the index to these organisms is usually low at the onset of the attack and rises rapidly as convalescence occurs, whereas chronicity is as a rule synonymous with persistence of a subnormal index.

Since it appears, then, that the opsonic index gives a very good idea of the progress of the case in catarrhal disease of the respiratory tract, it 
may well be asked-Why seek other methods of controlling dosage and intervals? The answer to this is that index determinations are tedious and troublesome to the medical man, and especially is this the case when certain strains of $M$. catarrhalis and pneumococcus have to be employed, and much more expensive to the patient than most can afford in these or any other times. The accuracy of index determinations, again, cannot always be relied upon, nor do they always give a true picture of the progress of the case : a high index especially does not always indicate progress, though a low index does usually connote a failure to improve on the part of the patient. Let us, then, consider what other guides we can substitute for the opsonic index.

(I) In such cases as exhibit prrexia we have seen that index and temperature run an inverse course to each other, so that despite the

CHART II.

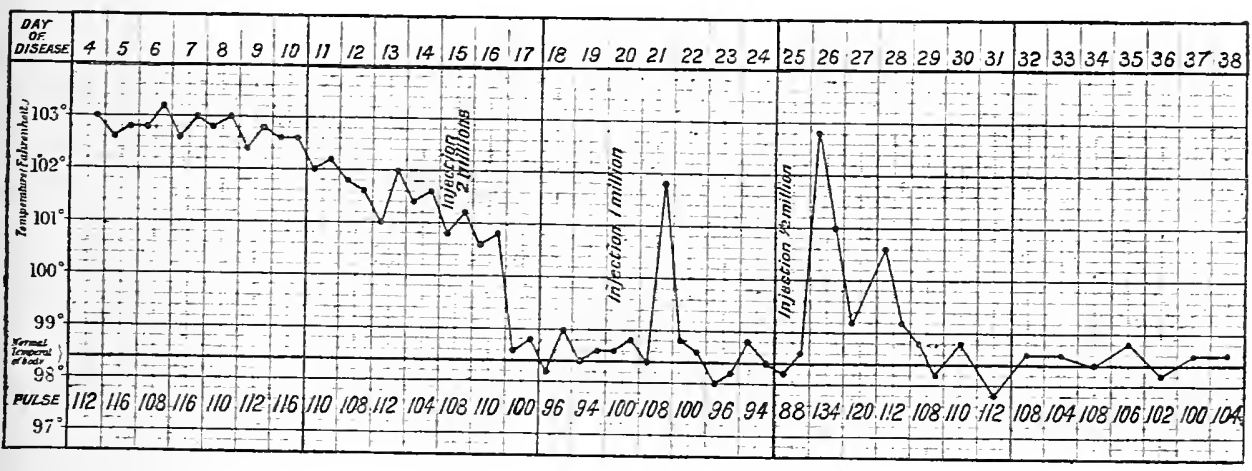

fact that temperature is considered to be a measure, not of immunising response, but of toxæmia, the curve may receive the following interpretation:

A rising temperature indicates increasing toxæmia and a falling opsonic index; if the tissues be capable of adequate response to the various stimuli, a subsequent fall in the curve will indicate diminishing toxæmia and rising index. This, moreover, is but a partial statement of the truth, and I cannot but feel that far too much stress has been laid on the temperature curve as a measure of the toxæmia. The temperature curve is much more than a measure of the toxæmia; it is compounded of many factors, one of which is the stimulus to the formation of various immune bodies. The toxæmic effect of a dose of half a million $B$. typhosus in the form of a raccine must be practically. negligible; none the less such an administration may be followed by a rise of temperature of $4^{\circ}-5^{\circ} \mathrm{F}$. (see Chart $\mathrm{II}^{*}$ ). Even granting that the body cells of this case were hypersensitive to the typhoid toxin, the

* Reproduced from vol. i, No. I, Joursal of Vaccine Therapy, after Dr. Wallis. 
pyrogenic effect can hardly be interpreted as due solely to the poisoning action of the bacterial toxins in the vaccine upon these cells; rather was it due to the stimulating effect upon the metabolic processes of the cells whereby antibodies are generated. The curve of antibody formation will therefore lag somewhat behind the temperature curve, the result being that with an effective stimulus the temperature will have already fallen by the time that the antibody curve has risen to an appreciable extent. The pulse-rate I take to be more nearly a guide to the toxæmic effect of the infection upon the tissues generally and the higher nerve centres in particular, but it, too, affords a rough measure of the immunising effect. It is quite rare to find a dose of vaccine producing any local effect upon a lesion which has not resulted

Chart III.

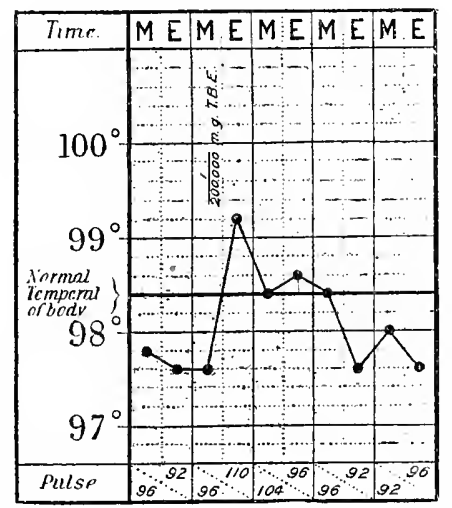

CHART IV.

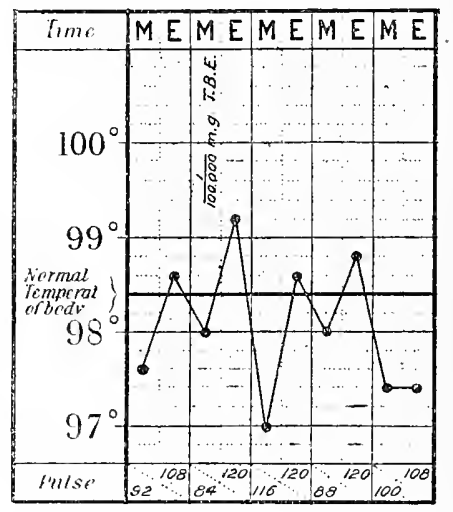

Charts Illustrating Rise in Temperature, Pulise-Rate and Opsonic Index after Therapeutic Doses of Tuberculin (Ian Struthers Stewart).

in a prior acceleration of the pulse-rate. In short, the usual result of a therapeutic inoculation is to produce a slight rise of temperature and an accelerated pulse-rate succeeded by a diminution in these and a rise in the curve of opsonin and such other immune bodies as it is at present possible to measure (see Charts III and IV).

(2) The rise in temperature and acceleration in pulse-rate may be accompanied by a slight feeling of malaise and mild headache, which in turn are succeeded by a feeling of increased general well-being and improved appetite; the former symptoms correspond to a lowered resistance, the latter to an increased production of the immune bodies.

(3) The effect upon a local lesion is the production of hyperæmia in and around it, the immediate result of this being, perhaps, slight increase of pain and of secretion or discharge. This increased blood supply is especially easy to observe directly in the case of such lesions as tuberculosis of the iris and larynx, lupus, and furunculosis. In 
catarrhal affections of the respiratory tract there may be a temporary increase of cough and secretion; the latter may be measured, and stethoscopic examination of the chest will reveal moist sounds where none before existed, or increased moist sounds where these were already present ; dry sounds, likewise, may be caused to appear or increased in number and in volume. Subsequently the inverse change will take place in regard to all these. There will thus be an alteration produced in the character both of the fluid and cellular constituents of the secretions. How these observations may be utilised in controlling the course of therapeutic immunisation will be fully dealt with later (see pp. 9I-95 and Charts $\mathrm{V}-\mathrm{XI}$ ).

(4) A change may also be observed in the bacterial contents of the secretion and in the phagocytic powers of the cells. The bacteria may be diminished in actual numbers, found of diminished power of growth in culture, or be seen agglutinated together or undergoing lysis, whilst phagocytosis may be found to be active where previously it was nonexistent.

(5) As immunisation is conducted to a successful issue, the patient may experience an increased sense of well-being, gain appetite and weight, experience less discomfort from the symptoms, and exhibit an obrious improvement in the state of all the tissues.

(6) The reaction of the tissues in immediate proximity to the site of inoculation is held by some to be a measure of the immunising response; this is to some extent the case, but so many complicating factors are concerned that in the majority of instances I think it should be neglected. The nature, mode of preparation and rolume of the inoculum, the amount and nature of the antiseptic contained therein, the density or laxity of the tissues into which the inoculun is introduced, all influence the degree of local reaction. The vaccines of some bacteria always produce a marked local reaction; some are more toxic if heated during manufacture, others less so: a large volume of diluting fluid and high percentage of antiseptic increase the local reaction, whilst the introduction of the inoculum into scanty subcutaneous tissues or just beneath a layer of fascia is sure to be provocative of more reaction than if the inoculation be done into abundant subcutanesus tissue or muscle substance.

In view of the frequency with which I am asked the question, "What constitutes a reaction to a therapeutic inoculation?" the following table may prove of service:

Table of Reactions Indicative of Efficient, but not Excessive Dosage. I. Slight temporary fall in opsonic index succeeded by a more marked and more permanent rise. 
2. Rise in the curve of agglutinins, lysins, etc., which may be preceded by a slight temporary fall.

3. Rise in temperature of $\mathrm{I}^{\circ}-\mathrm{I}^{\circ} 5^{\circ} \mathrm{F}$., or in pulse-rate of $\mathrm{IO}-20$ beats, followed within twenty-four hours by a fall, and steadying of the same at a more satisfactory level.

4. Slight feeling of malaise or headache, passing off within eighteen hours, and succeeded by a feeling of increased well-being and better appetite.

5. Increased hyperæmia around the lesions, with resultant increase of pain and in the amount of secretion, passing off in twelve to twenty-four hours, and being succeeded by increased comfort and diminished secretion. The amount of secretion may be measured, or its presence estimated by stethoscopic examinations. The swelling and reddening of the local lesions are succeeded by diminution in size and a more healthy appearance.

6. Improvement in the nature and degree of the bacterial content of the secretion and impairment of their vitality as observed in cultural experiments.

From this it will readily be understood that a marked and prolonged fall in opsonic index, or in the amount of lysins or agglutinins, a rise of temperature of $2^{\circ} \mathrm{F}$. or of pulse-rate by 20 beats, which fails to fall within twenty-four hours to a level lower than that previously existent, pronounced headache, malaise or vomiting, aggravation of local symptoms which is not speedily converted into an amelioration, or impairment of the patient's general condition are each and sundry indicative of excessive dosage. On the other hand, the failure to produce any apparently unfavourable or unpleasant prelude to an immediate improvement is indicative, as a rule, of a dosage too small to produce other than a very temporary good effect. At times the patient's condition may be such that any stimulus other than a minimal one cannot be applied without some risk; such cases, however, are relatively few, and as experience accumulates the fact becomes increasingly clearer that minimal stimuli applied at infrequent intervals are apt to produce a condition of anaphylaxis or hypersensitiveness, so that toleration to higher dosages and a high degree of immunity become difficult to obtain. Dosages, on the other hand, productive of moderate reactions and repeated at not too infrequent intervals are devoid of risk, and by their aid a high degree of immunity can be the more speedily established, provided, that is, that the tissues are capable of response to such stimuli. The importance of this point I shall explain more thoroughly in the section devoted to the vaccine therapy of pulmonary tuberculosis.

Finally, attention may be drawn to the following points in dosage : 
(I) That when a vaccine has been prepared some time the bacteria necessarily will sink to the lowest level of the bulb, and if the contents be not thoroughly shaken a mere fraction of the bacteria may be withdrawn from the bulb and introduced into the patient, who thus receives a much smaller dosage than was intended.

(2) That so long as a given dosage produces adequate reaction and subsequently an improvement in the condition any increase is not advisable ; unnecessary increase of dosage cannot be too strongly deprecated.

\section{IV (b) Special Considerations in the Conduct of Vaccine Treatment of Respiratory Catarrhs.}

IT was in May, I90, that I performed upon myself the first administration of a vaccine for the cure of chronic respiratory catarrh; since that time vaccine treatment has been extended more freely in the direction of catarrhal disorders of the respiratory tract than perhaps in any other, and judging from such reports as have been published or have reached me privately $I$ think it may be safely said that in no other disorders are such uniformly successful results achieved.

In the first part of this chaper I have dealt with the preliminary considerations that must be kept in mind before vaccine treatment is to be begun. In the event of a sufferer seeking relief from an acute attack of nasal catarrh or laryngitis it is obviously difficult to postpone special treatment for that attack until such time as due attention has been paid to any abnormalities; none the less, proper counsels should not be deferred, and if the attacks be not of undue severity, I endeavour to prevail upon the patient to employ more stereotyped and general treatment during that attack, then to have abnormalities treated and finally to return for immunisation. With chronic infections the necessary preliminaries should be insisted on as I have already said. Should no abnormal conditions be existent or should they have been already put to rights our procedure should be as follows :

(I) By means of suitable questions endeavour is made to determine whether the attacks from which the patient suffers are always due to the same micro-organism or micro-organisms. How this is done I have already partly indicated in the issues of the Lancet for November 28th and December 5th, IgoS, and February I3th, I9og. The most characteristic effects of infection by each catarrhal organism briefly are as follows :

(i) That by the $B$. septus usually begins as dryness or tickling of the soft palate, the inflammatory condition extending within twentyfour hours to the nasal mucosa so that sneezing and discharge of thin nasal mucus result, and to the pharynx, so that there is some pain in 
swallowing. As the infection practically never extends to the larynx and lower respiratory passages, cough, other than pharyngeal, is not present, and expectoration scanty; as the Eustachian tube appears but rarely to be involved deafness and noises in the ear are not produced; as the accessory air-cells as a rule escape attack, sense of fulness and referred head-pains are also absent. I think the antrum does frequently become involved, but the process is a mild one, produces little or no symptoms and rapidly clears up; on the other hand, as the nasal mucosa bears the brunt of the attack, sneezing, copious discharge, considerable swelling of the tissues, loss of smell and taste are the prominent features; toxic absorption being slight, headache, malaise and pyrexia are also slight. After three or four days the acute stage rapidly subsides, and a subacute stage, characterised by thick but not very purulent mucus, persists for perhaps an equal period. True chronic infection of these parts by the $B$. septus is quite rare; when it does exists it is revealed. rather by a bacteriological examination and the recurrence of acute attacks than by any chronic symptoms.

(ii) The Micrococcus catarithalis, on the other hand, may initiate its attack at any part of the respiratory tract and so produce anything from a purely nasal catarrh to a capillary. bronchitis. Characteristically I think it begins with an inflamed feeling of the fauces and nasopharynx; extension into the nose and larynx soon ensues, so that there is profuse thin discharge from the former and expectoration of thin, colourless, rather watery mucus from the latter; impairment of voice, cough, and sore feeling in the trachea are necessary accompaniments, the Eustachian tube becomes frequently involved, so that some deafness and noises in the auditory passages result. Infection of the accessory sinuses is the rule rather than the exception, so that sense of fulness and perhaps referred pains may be felt; toxic absorption produces headache, malaise and some rise of temperarure; while erythema and petechiæ have been also noted. At the end of three to four days the subchronic stage is entered on by the infection in the nasal passages and may persist for weeks so that profuse muco-purulent mucus may be frequently expelled; at the same time the lower respiratory passages may be in turn involved, with the final production of a troublesome and persistent but not very acute infection of the bronchi and bronchioles from which copious thin, not very tenacious or purulent mucus is voided.

Infections by the $M$. catarrhalis have a decided tendency either to become truly chronic or to frequently recur. At times they very closely simulate infections by the $B$. infuenza.

(iii) The Micrococcus paratetragenus may behave precisely like the $M$. catarrhalis; but the experience gained from two epidemics seems to 
indicate that the larynx is its favourite point of attack. The first symptom may be huskiness of voice followed in a day or two by a dry paroxysmal cough, which may result in the expulsion of a tiny blob of clear but exceedingly tenacious mucus; subsequently the infection may extend simultaneously into the upper and lower passages. Secretion is rarely profuse and practically never even muco-purulent; involvement of the accessory sinuses I have never seen. Persistent dry hacking cough is its main characteristic.

(iv) The bacillus of Friedlander group. - This organism usually confines its attentions to the nasal passages and their adnexa. The first symptoms of its activity are sneezing, some malaise, slight headache and loss of smell. The nasal discharge soon becomes exceedingly profuse; usually it is clear and colourless, but when the antrum or sinuses become involved it takes on a muco-purulent character. As the Eustachian tube almost always escapes attack deafness and noises are not experienced; the throat does not become inflamed, nor the larynx and trachea involved; sore throat and cough are therefore absent. Rarely in the adult, but more commonly in the child, the bacillus of Friedlander may make its way into the bronchi or pulmonary tissues, setting up bronchitis on the one hand and broncho-pneumonia on the other. It is the variants of this organism which more often set up these latter conditions, while those forms which resemble it very closely, except that they possess motility, are those which are most prone to involve the Eustachian tube and accessory sinuses. Infections by the bacillus of Friedlander are especially apt to assume chronicity.

(v) The pneumococcus, like the M. catarrhalis, is capable of involving each and every portion of the respiratory tract. The most characteristic attack begins as follows: For a day or two there is a feeling in the larynx and upper trachea as if sand-paper had been applied to the mucous membrane ; cough is very harsh and dry ; convulsive efforts may finally succeed in expelling a blob of mucus very similar to that characteristic of the $M$. paratetragenus except that it may possess a yellowish tinge. At the end of the second or third day the infection has begun to spread upwards into the naso-pharynx and down the trachea, so that by the third or fourth day the pharynx and trachea may feel sore, while expectoration and nasal discharge become profuse and soon assume a muco-purulent, and later a purulent yellow look and possess slight tenacity. Toxic absorption may produce considerable malaise and headache and some rise of temperature; involvement of the sinuses, a sense of fulness and referred head-pains; extension to the chest, moist sounds in the bronchi and bronchioles, and muscular or pleuritic pains are common. Finally, the alveoli may be attacked and a condition of definite pneumonia established. This is a description of a typical pneumo- 
coccal invasion of the respiratory tract; occasionally, however, the nasal mucosa is the first attacked; sometimes it is the bronchial mucosa, or yet again the pulmonary cells; sometimes the attack may be localised entirely to the nasal passages, sometimes entirely to the passages of the chest-an excellent example of this last is afforded by the condition known as acute suffocative catarrh of Laennec.

(vi) The B. influenza produces the most protean symptoms, but it must be borne in mind that usually associated with it is the pneumococcus.

An attack of true influenza is usually ushered in by extreme malaise, headache, joint and muscle pains, and pyrexia, perhaps with rigors. Before any respiratory symptoms have developed considerable upset of the digestive system may be produced, while the nerves may be so involved that neuritis, diffuse or local, or herpes zoster may appear. In such instances the infection is probably a systemic one, subsequently to be localised in the pulmonary tissues.

Sometimes infection is confined to the nasal passages and their adnexa, and does not extend to the lower respiratory tract; in these instances infection of the antrum, of the ethmoidal, frontal and sphenoidal cells, and of the Eustachian tube is very common, and while in the majority of instances chronic infection thereof does not result, in a by no means inconsiderable number chronicity does ensue.

(vii) The streptococcus group, while certainly concerned in such conditions as chronic post-nasal catarrh, bronchitis and asthma, and often in infection of the sinuses, and sometimes in the later stages of catarrhs of the upper passages, yet produces no symptoms which enable us to fix on one of its members as certainly concerned in any of these processes; to bacteriological research must be left the determination of this question in any given case.

Armed, then, with the knowledge of these points of differential diagnosis, it becomes a relatively easy matter, by means of appropriate questions, to elicit from an intelligent patient whether all attacks of respiratory catarrh from which he suffers are set up by the same organism or organisms; for it is the rule, rather than the exception, not only that susceptibility to several catarrhal organisms should exist, but that two or more of these should make a simultaneous attack; a little practice will enable one not only to say, for instance, that a patient is suffering from a pure $B$. septus infection, but that there is a combined attack by the bacillus of Friedlander and $M$. catarrhalis, or pneumococcus and B. infueñce. But it may be said, What help do we receive from this more or less guess-work diagnosis? The answer is this: Not only does this guess-work diagnosis sometimes prove more accurate than a bacteriological examination of the secretions, for the sample taken may be 
ill-chosen, or the true infecting organism not yet be in process of free discharge and so escape detection until subsequent examinations, but it also enables us to answer the question every patient is sure to put, "Will this vaccine not only help to free me from the present attack but also protect me from future ones?" Obviously a vaccine made during an attack due, say, to the pneumococcus and M. catarrhalis, will in no way help to ward off subsequent attacks by the B. septus or B. influenzer; and careful investigation on the lines indicated will aid in the determination of the patient's liability to subsequent attacks by these organisms. Personally, if I decide that my patient is liable to infection by organisms other than those of which I am about to prepare the autogenous vaccine, I then lay before him these alternatives:

(I) To include with his autogenous strains heterologous vaccines of such other catarrhal organisms as he appears susceptible to.

(2) To confine my attention for the time being to the preparation of the autogenous vaccine; to inform the patient that inasmuch as this will only protect against infection by the contained micro-organisms he will remain susceptible to other varieties, and in the event of being attacked by these it will be necessary for him to return and afford opportunity for the preparation of a second autogenous vaccine to combine with the former.

The preparation of the vaccine.-Having thus made a tentative diagnosis and decided the precise object that it is desired to achieve, specimens are taken with the precautions indicated on pp. 8-Io. Smears are prepared, stained, and examined by the methods already outlined for bacteria and cellular contents, while cultures are made on the various media indicated by this preliminary examination. After adequate incubation at $37^{\circ} \mathrm{C}$., it may be for one, two, or three days, colonies of the various organisms of which it is desired to prepare vaccines are picked off and inseminated upon fresh culture-plates in order to secure pure cultures. Here let me just mention that inasmuch as great difficulty sometimes presents itself in the growth of some organisms such as the $B$. influenza in subculture, that it may be requisite to employ special enrichment of the culture media (p.32), and in addition to retain the original culture-plates until it may be seen whether growth will or will not then take place; in the latter event the original plates must be employed for the preparation of the vaccine of the delicately growing micro-organism; as a rule the differential standardisation of such a mixed vaccine presents few difficulties, and various little devices which ingenuity will suggest may be employed in face of these. Suppose, for instance, that it be desired to prepare vaccines of M. catarrhalis and $B$. influenza, and that the latter has refused to grow in subculture, after thirty-six to forty- 
eight hours' incubation of the original plates the colonies of both organisms will have well developed. Subcultures are taken of the $M$. catarrhalis, and a little $o^{\circ} \mathrm{I}$ per cent. aqueous salt solution is added to the growth and emulsification performed with light touches of a bent glass or aluminium rod; the growth of $B$. influenza will be readily detached, whereas the $M$. catarrhalis will adhere to the medium with considerable tenacity; the colonies, moreover, even if some do become detached, will not readily disintegrate. The mixed emulsion is then pipetted off and centrifugalised at a slow rate; this will amply suffice to throw down the great bulk of the $M$. catarrhalis and leave the $B$. influenze in suspension. The emulsion is then carefully pipetted off from the sediment and standardised in the usual manner (VACCINE Therapy, third edition, p. 40). If, in addition, a vaccine of the $M$. catarrlalis be desired the subcultures are to be employed. As I have already said, emulsification of the $M$. catarrhalis, and sometimes of the $M$. paratetragenus and certain strains of streptococcus, is by no means easy to perform. The following hints may prove of service: (I) Make the culture medium rather less solid than usual, so that there is abundant water of condensation; (2) incubate for not more than twelve to fifteen hours; (3) emulsify by means of a bent aluminium rod which has been slightly roughened by means of coarse sand-paper or a fine file; $(4)$ do not add further fluid to the culture plate until a rough emulsion has been made in the water of condensation; (5) pipette off a little of this emulsion, and place in a strong test-tube with eight to ten glass beads, and try whether a salt solution of $\mathrm{O}^{\prime} \mathrm{I}$ per cent. or $\mathrm{I}^{\circ} \mathrm{O}$ per cent. concentration aids emulsification; also see whether heating the tube and contents in a water bath for a few seconds at $70^{\circ}-75^{\circ} \mathrm{C}$. is an additional help. Having ascertained the conditions which most favour emulsification, employ these in the treatment of the remainder of the thick emulsion; dilute the product with salt solution of the appropriate strength and at the appropriate temperature, centrifuge rapidly for a few seconds, pipette off the upper portion and immediately standardise.

Where treatment of a case is to be conducted with the vaccines of more than one organism, mixture may be made of the several vaccines after standardisation in the desired ratios; the bulk of the inoculum, and so the amount of local reaction, is thus kept at a minimum. As regards the method of sterilising the vaccines, it may be said that the addition of $0^{*} 3^{-0} 4$ per cent. tricresol to many vaccines of concentration of not more than 2000 million organisms per c.c. will secure complete sterility in twenty-four hours; with some bacteria! this concentration may be greatly exceeded; with others sterility is secured only with much lower concentrations. 
In this way heating of the racçine may often be aroided, but it is a fact that occasionally a vaccine sterilised by antiseptic proves to be of less service than one sterilised by moderate heat for not too long a time; this especially I have found to be the case with certain strains of pneumococcus and streptococcus; more often the reverse holds good.

If heat be used to sterilise the vaccine a temperature of $56^{\circ}-5 \mathrm{~S}^{\circ} \mathrm{C}$. for thirty to forty-five minutes will as a rule suffice, unless, of course, contamination with sporing organisms be present; every care should be taken to exclude these, but should any find their way into the emulsion from the air or in the diluting fluid, their multiplication may be completely inhibited by the addition of $0^{\circ} 3$ per cent. tricresol or carbolic acid to the emulsion. Cultural tests should always be performed upon the finished product, and if any living bacteria are detected their precise nature must be determined; if they consist of an organism which under any conditions is capable of exerting a pathogenic action, the vaccine must of course be discarded and a fresh one prepared; on the other hand two or three spores of $B$. subtilis or $B$. mesentericus in a dose of vaccine are quite devoid of danger; at the same time they are better absent. As a rule heating the vaccine to a higher temperature than that indicated impairs its efficiency and keeping properties, and increases its toxicity.

Some of each vaccine in the highest concentration should be stored for future need, for, as immunisation proceeds, some organisms may disappear before others, and the administration of the corresponding vaccine be unnecessary; or, again, the proportions in which the several have been blended may not prove to be the best.

The administration of the raccine.-While it is the general consensus of opinion that the introduction of a vaccine directly into the substance of the tissues has decided advantages over oral administration as advocated by Latham, yet at times the latter procedure would seem to offer certain advantages. It a discussion before the Therapeutical Section of the Royal Society on February 2oth, IgI2 (vide Proc. Roy. Soc. Med., March, IgI2, Ther. Section, p. 55), Latham outlined his position more clearly, and remored certain misapprehensions. $\mathrm{He}$ would now appear to uphold this procedure only when there are definite obstacles to the subcutaneous administration, and strongly urges the necessity of giving the vaccine upon a perfectly empty stomach early in the morning, and in a liquid isotonic with the tissue fluids and free from all antiseptics. Stomach derangements or a furred tongue are contra-indications to the procedure. While there is considerable difficulty in understanding how the administration by the mouth of small doses of a killed culture can produce immunity in a person who is constantly swallowing, both on an empty stomach and otherwise, large doses of his own micro-organisms in a fluid of his own secretion, 
yet I must admit that one or two cases have come under my own observation in which it appears that immunity has been so produced. In such instances, therefore, as the following, (I) when the subcutaneous administration of even tiny doses produces an undesirable reaction, (2) when the patient refuses to submit to puncture, or persistently collapses or faints under the inoculation-and I have met with several such cases, (3) when the patient is going out of reach of medical men, and desires to continue treatment, (4) that of infants or of very young children; the oral administration of the vaccine may be most desirable. With these exceptions inoculation is the better procedure, for no doubt can then exist of the actual dose of vaccine employed to stimulate the tissues. Hypodermic injection having been decided on, the question of site remains to be considered. Intra-muscular inoculation has been advocated as possessing advantages over subcutaneous ones; it is held to be less painful, and cause less tenderness, while the bacteria are more readily absorbed, and therefore produce more speedy immunising response; a disadvantage is the greater risk of puncturing a bloodvessel, and of introducing the inoculum therein. This, however, is not a very real danger, for the endeavour to withdraw the piston will soon indicate whether the needle-point is within a vein. Subcutaneous inoculation is that most commonly practised; the best sites are where subcutaneous tissue is lax, and no pressure by clothes, or otherwise, will be exerted upon the site. For these reasons the tissues of the arm or leg should be avoided, whereas the following localities are highly suitable: (I) the upper buttock $2-3$ in. from the medium line; (2) the flank or abdomen in well-covered individuals; (3) $1 \frac{1}{2}-2$ in. below the centre of the clavicle.

The procedure is as follows: take an all-glass syringe with sharp, clean needle, best of platinum-iridium, in default of this of steel. Sterilise the glass parts separately by boiling in distilled water, a platinumiridium needle by boiling, or passage through a flame, a steel needle by boiling in methylated spirit, and put the parts together by means of sterile forceps; shake up the bacterial emulsion thoroughly, nick the neck of the containing bulb with a file or glass-knife and snap it off; invert the bulb over the needle-point, and aspirate the contents into the syringe. Sterilise the skin of the patient at the selected spot with iodine, Io per cent. lysol, or alcohol and then ether, pick up a good fold of tissue firmly between left thumb and forefinger, boldly plunge the needle into the tissues, subcutaneous or muscular, as is wished, and slowly introduce the inoculum therein; withdraw the needle quickly. These precautions amply suffice to preclude septic infection, and ensure the minimum of discomfort. 


\section{CHAPTER VI.}

IV (I) VACCINES IN THE TREATMENT OF NASAL AND POST-NASAL CATARRH, TRACHEITIS AND LARYN. GITIS, AND INFECTIONS OF THE ACCESSORY SPACES.

In the treatment of ordinary catarrhs the following points require consideration :

(a) At what period of an attack to begin immunisation.

(b) At what time of day to perform inoculation.

(c) The initial and subsequent dosages and intervals.

(d) General treatment.

(c) Advantages of specific treatment and results to be expected.

Let us consider these seriatim.

(a) The period of an attack at which to commence immnunisation.Formerly I used to consider it inadvisable to inoculate during the first three days of an acute attack and thought it wise to defer interference until the inception of the subacute stage. This attitude was brought about by observing the effects produced by the dosages I then employed. Now I know that the occasionally unpleasant sequelæ were due entirely to excessive dosage, and am sure that the earlier we begin specific treatment the better. How soon this may be done depends on several factors, such as-(I) the stage at which we are consulted; obviously if we are not called in at the inception we cannot inoculate at the inception; (2) does the patient insist upon the use of an autogenous vaccine, or will he content himself with a stock one to begin with? In the former case at least twenty-four hours will be consumed in the preparation of the vaccine. In the latter event it will depend partly upon the accuracy of the patient's description, partly upon our own skill in making therefrom the correct differential diagnosis. If there be any doubt upon this point a combined vaccine of the various catarrhal organisms, or at all events of such of them as are known to be concerned in the production of the existing epidemic, must be employed. (3) Is the patient able to present himself, 
or can a visit be paid at the time of day suited for the inoculation, and is it possible for him subsequentiy to observe the necessary precautions? (see section $b$ ). If these conditions cannot be fulfilled, then it is better to wait for two to three days until the subacute stage has been entered on.

(b) At what time of day to perform inoculation.-Acute catarrhs are an infectious disease, fraught with possible dangers not only to the patient but to those with whom he comes in contact. If this were adequately realised their incidence and mortality would be much reduced. No one suffering from acute catarrh should attend a public gathering or sneeze into the air, but into the folds of a handkerchief moistened with a few drops of formalin, or above all allow anyone to come in contact with him to whom an acute catarrh would prove of especial danger, such as the very young, the old and infirm, those suffering from impairment of the heart's action, or some other respiratory disease. Per contra, bed is the ideal place, in a well-ventilated but warm room, attended by a suitable nurse, who, when in attendance, should cover the mouth and nose with a pad of lint upon which a drop or two of formalin has been sprinkled. If the patient can follow these instructions inoculation may be performed at any time of day, but best at night between 7 and Io p.m., as then the least interference with the night's repose is brought about.

In the event of a commencing cold there is no particular objection to the patient making call upon his way home after the day's work is done. By immediately going home, eating a light dinner and retiring early he will avoid all danger of exacerbation of the attack. A similar procedure is suitable in the case of chronic colds, but none the less it is always preferable to give a first inoculation when the patient is in bed. Subsequent doses may be given any time after 3 p.m. provided the patient is proceeding home and will follow the precautions above described; still, the later in the day the better; personally I prefer between 5 and 7 , the earlier time in winter and the later in summer.

(c) The initial doses of the various organisms which may be safely employed in the first few days of an acute catarrh are as follows: B. septus, 50 millions; M. catarrhalis, 25 millions; M. para-tetragenus, 25 millions; pneumococcus, 25 millions ; B. influen $\sim a, 50$ millions; $B$. of Friedlander, 50 millions. These may be used singly or in any combination. The effects to be expected from such an administration are as follows: During the ensuing twelve to eighteen hours there may be a slight exacerbation of all the symptoms, slight headache and malaise, acceleration of pulse by ten to fifteen beats, and rise of temperature of $\mathrm{I}^{\circ}-\mathrm{I} \cdot 5^{\circ} \mathrm{F}$. Any one or more of these constitute a reaction. The subsidence of these may be very rapid, and within twenty-four hours the 
improvement in the patient's symptoms and feeling of well-being may be quite marked. The absence of any reaction or of improvement within forty-eight hours is indicative of the immediate advisability of giving a double dose; failure to react to this almost certainly points to faulty diagnosis of the infecting organisms, and fresh smears and cultures should at once be made. Should the patient, on the contrary, react in any of the above ways, re-inoculation may be deferred until retrogression occurs-but this is hardly a good practice-or, better, be performed upon the fourth or fifth subsequent evening. The indications, then, both of dosage and intervals consist in the clinical signs and symptoms and in the production of a mild reaction. The inadvisability of ever increasing dosage so long as adequate responses are made should be ever borne in mind, while the possibility of an incorrect diagnosis of the infecting organisms, even when this has been based upon a complete bacteriological examination, is always suggested by failure to respond in any way to the double dosage above indicated. Failure to continue or maintain improvement may be due either to inadequate stimulation, or to increased vitality on the part of one of the infecting agents in a mixed infection, and so to inadequate dosage of that particular vaccine, or to a fresh infection by another microbe ; in which particular direction the fault may lie is usually elucidated by a bacteriological examination.

In the case of chronic catarrhs the same initial dosage as for acute infections may be employed; personally, I prefer to begin with the double dosage, as reactions are both milder as a rule and more difficult to produce. The intervals between dosages are to be controlled by the reactions, clinical symptoms, and bacteriological examinations. The suitable interval I find to vary between seven and ten days. As before, it is inadvisable ever to increase the dosage so long as either (I) a distinct general reaction is produced, or (2) the patient continues to improve after each administration.

A chronic catarrh is always best treated with the autogenous vaccine, and the following points should always be clearly borne in mind: (I) That as one variety of a micro-organism is eliminated another may come to the front; for instance, in a mixed pneumococcus $-B$. influenze infection it is no uncommon experience to find that as the $B$. influen $\approx$ a disappears the pneumococcus comes to the front; the patient, so far from making marked improvement, may even appear to be becoming worse. Careful examination of smears and cultures will reveal this, and indicate the necessity of maintaining the dosage of $B$. influeña, but of increasing that of the pneumococcus.

(2) That a fresh acute infection of some other prevailing organism may be incurred, and mask the real improvement that is being 
produced with regard to the chronic infection. Here, again, careful examination of smears and cultures will prevent misconception, and indicate the necessity of preparing a fresh vaccine. A good example of a case like this is related by Dr. Roger Smith (Journal of Vaccine Therapy, vol. I, No. 3, p. 95).

Briefly, then, increase of dosage is indicated by failure-

(I) To obtain a slight immediate reaction;

(2) To secure improvement in the clinical condition after any dose, provided that the possibility of $(a)$ increased activity on the part of one of the infecting micro-organisms, (b) fresh infection by another bacterium, has been eliminated.

Re-inoculation is indicated by slight retrogression or failure to continue to improve on the part of the patient; experience has shown that the interval between inoculations should be between seven and ten days.

(d) General treatment.-The preliminaries to any course of vaccine treatment which should be taken have been already fully described; in addition general treatment on more or less stereotyped lines should also not be neglected. For instance, in cases of acute catarrh the patient should be confined to bed in a warm, well-ventilated room, the diet restricted in regard to meat, fish being an appropriate substitute; careful attention should be paid to the bowels, and fluids limited if the mucoid discharge be unduly copious. It is most instructive to observe the effect, say, of a cup of tea upon the nasal discharge when fluids have been restricted for twenty-four hours; within a very few minutes of the drink the secretion of mucus may be increased many-fold, so that the discharge even drips away from the passages. Handkerchiefs should be sprinkled with two or three drops of pure formalin, and a silk one used to dry the nose; by smearing the orifices inside and out with a little resinol or boracic ointment painful excoriations may be obviated. A hot foot-bath of mustard and water and a hot-water bottle to the feet or side will increase the patient's comfort; a hot drink of lemonade or whiskey and lemon, followed half an hour later by a dose of the following prescription by Dr. Burney Yeo, will promote diaphoresis and the action of the lidneys, and often mitigate or shorten the attack:

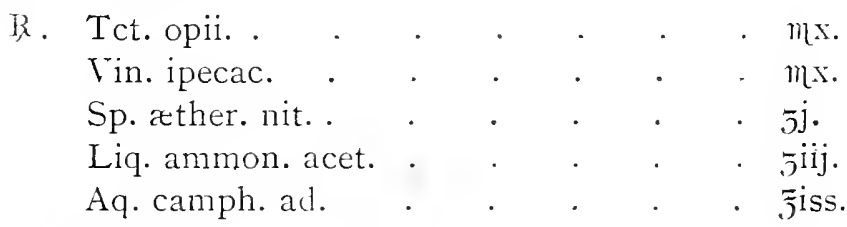

M. et.f. $h$. 
An additional blanket should be put upon the bed, and in the event of copious sweating being produced the patient should be urged not to withdraw the hands from beneath the covering, nor to arise in the morning before cooling off by degrees; the above prescription may be repeated with advantage immediately on waking in the morning.

When laryngitis or tracheitis is present nothing does so much good as the application of a cold water compress.

Sprays and antiseptic lozenges, such as formamint, conduce to comfort when pharyngitis or tonsillitis is present, but in the value of ammoniated tincture of quinine I am a profound disbeliever.

Inhalations of steam saturated with the vapours of pure eucalyptus or cinnamon oil or of Friar's balsam sometimes prove of service, but rather in affections of the lower respiratory tract than of the upper.

\section{(e) Advantages accriing from Spccific Treatment.}

(I) Acute attacks may be aborted altogether or greatly shortened in duration. In the great majority of instances the patient, if seen at the inception of an attack, should be perfectly able to resume his duties on the third morning after the first inoculation; when confinement to the room for two days is hardly possible it is perhaps advisable to begin with a double dosage; the reaction will be greater, but so will the immunising response, and this should be well established after an interval of eighteen hours.

(2) The risk of complications is greatly minimised. As I have aiready said, pneumococcal infections usually begin somewhere about the larynx, influenzal ones probably higher up the tract. Vaccine treatment will almost certainly prevent extension downwards, and I think I am understating the truth when I declare my belief that at least 90 per cent. of the cases which are commonly stated to have died of pneumonia following upon influenza could have been saved by the exhibition of a vaccine at the commencement of the attack; even in the later stages, when extension to the chest has already occurred, more than 50 per cent. of the deaths should be likewise obviated. As I wrote some years ago, it will be a very long time before adequate recognition is accorded to this fact; meanwhile thousands of valuable lives will be needlessly sacrificed at every epidemic.

(3) Chronic sufferers who have tried almost every other form of treatment will be either cured completely or afforded a considerable measure of relief.

(4) Subsequent immunity, more or less complete, may be secured 
against future acute attacks; the completeness of the immunity will depend-

(a) Upon the completeness of the vaccine: a vaccine containing, say, pneumococcus, $B$. septus and M. catarrhalis can hardly be expected to afford protection against the $B$. infuenza.

(b) The adequacy of the treatment.

(c) The choice of suitable times for subjecting the patient to a short course of treatment.

For the production of immunity against acute catarrhs an appropriate vaccine is the combined vaccine for colds of the Wimpole Institute, which is compounded of practically all the catarrhal organisms. It should be employed as follows: Inasmuch as the average duration of complete immunity when this is once established is about six months, two courses of treatment should be given in each year. In England the end of September or the beginning of October, and the end of January to the beginning of March usually usher in an epidemic of acute catarrhs. The patient should, therefore, present himself for treatment in the middle of September and the middle of January. A first dose of 50 millions of the combined vaccine for colds should be followed at ten-day intervals by a roo-million and a 250-million dose. Thus, by the end of the first week in October and the first week of February full immunity should have been established, and it may be anticipated with a certain degree of confidence that any prevailing epidemic may be escaped. Against the Bacillus influenza it is a difficult matter to secure immunity; probably a smaller dosage than Iooo or even 2000 million organisms will not prove efficient ; personally I always warn my patient that immunity against this organism cannot be assured.

Post-nasal catarrh is, as a rule, a chronic infection of the naso-pharyngeal space, and is connected clinically and ætiologically rather with pyorrhœa alveolaris and follicular tonsillitis than with catarrhal infections of the other portions of the respiratory tract; it is a very important cause of preventable deafness. I have in the past been perhaps somewhat lax in differentiating sufficiently cases of chronic post-nasal from chronic nasal catarrh. Accordingly I think that the figures given in Table VI on p. 46, taken from C. E. West's paper read before the Otological Section of the Royal Society of Medicine in February, I912, are probably more reliable than my own given in Table III, p. 44, which have been taken from cases of both nasal and post-nasal catarrh.

In virtue of the intimate relationship which exists between this complaint and pyorrhœa alveolaris and follicular tonsillitis the absolute necessity of careful search for these latter conditions and insistence 
upon adequate treatment if they prove to be present prior to engaging upon vaccine treatment of the post-nasal catarrh is sufficiently obvious. Only rarely is this condition due to infection by a single organism, when it is, the bacillus of Friedlander is probably the one concerned, mixed infection being the rule; streptococcus, pneumococcus, $M$. catarrhalis and Staphylococcus aureus may be present in any combination. Autogenous vaccines, therefore, are much more likely to prove efficacious than any stock one, the dosages to be employed are similar to those indicated for the treatment of chronic nasal catarrh; the intervals are also similar, and the progress of the case is also to be estimated by clinical symptoms and bacteriological examinations.

$\mathrm{My}$ own results have been particularly good; in the cases which I have treated for otological specialists, such as Dr. Greville Macdonald, Mr. Herbert Tilley and Mr. Seccombe Hett, the report by these observers has always been that the disappearance of the catarrh has been very striking. Unfortunately the influence upon the deafness has not been correspondingly good, the cause being probably the extreme chronicity of the cases. My own private cases have been equally satisfactory as regards the catarrh symptoms.

West's conclusions in regard to the efficacy of the treatment are wisely guarded, as his experiments have not yet reached finality. $\mathrm{He}$, however, states that "Friedlander infections seem to do well and catarrhalis quickly disappears under vaccine treatment. Staphylococcus aureus seems to be a favourable case for vaccination, and the streptococci certainly diminish or disappear in some cases. On the other hand the pneumococcus has so far defied my efforts, and has appeared to be just as numerous and just as effective in maintaining catarrhal processes after lengthy courses of vaccine as before them. It may be that fuller investigation will show a more successful method with pneumococcal infections, either by the use of still larger doses or by employing a vaccine from a specially virulent strain." In default of definite information as to dosage it is difficult to judge how far this surmise may be true; probably it is justified, my own experience being that ultimate dosages of 500 or even 1000 million pneumococci are sometimes requisite. As regards the second point I think the fault may possibly lie in the method of preparation of the vaccine. As I have already said, sometimes a pneumococcal vaccine sterilised by heat is devoid of immunising power while one sterilised by antiseptic alone secures an immediate response. At the same time I must admit that I also have at times found it impossible to prepare by any means an efficient vaccine from certain strains of pneumococcus.

The effect of vaccine treatment upon chronic Eustachian catarrh has been very striking so far as the catarrh itself is concerned, but 
disappointing as regards any marked improvement of the actual hearing; inasmuch, however, as all my cases have been ones of very old standing, as a rule of about twenty years or more, complicated perhaps by some degree of oto-sclerosis, and had been already subjected to all such other methods of treatment as the skill of wellknown specialists could devise, the production of even a slight degree of improved audition should, I suppose, be considered satisfactory; in perhaps 50 per cent. of my cases this has been obtained. The best effects have, however, been the prevention of acute outbursts of the Eustachian catarrh and the resultant maintenance of the hearing at a more steady level.

In acute otitis media vaccines appear sometimes to prove of very great service in clearing up the infection; there is, however, no little difficulty in estimating their precise effect ; for inasmuch as puncture or incision of the membrane is requisite for obtaining cultures, and this procedure, combined, of course, with such other measures as experience indicates, frequently of itself brings about a speedy cure, the good results seen after exhibition of a vaccine cannot with any degree of certainty be allocated in any particular case to this or that form of treatment. When, however, perforation of the drum has spontaneously occurred and the infection does not appear to yield to the more stereotyped methods of treatment, the effect of vaccine treatment can be better gauged. One case in which double otitis media, consequent upon an attack of scarlet fever, responded with striking rapidity and completeness to inoculations of the autogenous staphylococcal and streptococcal vaccines I have already recorded (see Lancet, September IIth, I909, p. 780). The membranes healed with hardly any trace of scarring and the hearing when examined six months later by a well-known aurist was found practically normal.

In more chronic cases it is, of course, easier to arrive at a just appreciation of the value of vaccine treatment. Good results have been obtained in infections due to the B. protens, B. pyocyaneus, $B$. coli, streptococcus, pneumococcus, staphylococcus; inasmuch as there is always a tendency for the infection to change in character, occasional bacteriological examinations of the secretion are very essential.

The case for vaccine therapy in otitis media, acute or chronic, may be fairly summarised as follows: It can do no harm, while it will almost certainly prove a valuable adjunct to other forms of treatment in hastening the healing process, limiting the extent of damage and consequent scarring of the membrane and fixation of the ossicles and in diminishing the risk of intra-cranial complications.

In acute infections the following initial dosages may be employed: 
Streptococcus, Io to 25 millions; pneumococcus, Io to 25 millions; staphylococcus, 50 millions; $B$. proteus, 50 millions; $B$. pyocyaneus, 50 millions; B. influenza, 50 to Ioo millions; M.catarrhalis, 25 millions. Higher initial dosages than these are not advisable unless very free drainage be maintained, for increased discharge and augmented pain usually ensue for twelve to eighteen hours. Undue haste in increasing subsequent dosages is also to be deprecated, for these cases appear to make good progress upon small doses. The usual interval is about five days or rather longer.

In chronic cases trial may be made with like amounts, but in default of progress augmentations may be made rapidly with little hesitation. I have myself used 500-million doses of $B$. pyocyaneus and Iooo-million doses of $B$. proteus with very good result and no illeffects.

In infections of the anirum and accessory spaces, we are confronted by no little difficulty in arriving at a just appreciation of the scope and value of vaccine treatment. As to the frequency with which involvement of one or more of the accessory sinuses occurs during attacks of acute rhinitis statistics are wholly lacking. Personally I think that it does occur in at least So per cent. of all cases, and that it is especially frequent in acute catarrhs due to the $B$. influenza, $M$. catarrlalis and pneumococcus. If this be so spontaneous cure must be very frequent. The rapidity with which an antrum full of pus can clear up is very striking. I have observed a complete shadow as seen by transillumination entirely disappear within thirty-six hours and not recur. The ease with which this can happen must obviously largely depend upon the position of the opening with regard to the floor of the cavity. If this be near the floor evacuation is easy; the higher up it is the greater the obstruction to natural drainage. The poorness of the blood supply and the scanty amount of tissue covering the bony walls make it difficult to understand how the copious exudate is formed and the mechanism whereby absorption occurs of the residue which fails to drain away. The fact remains that several drachms of pus may be secreted daily and that spontaneous evacuation and absorption may occur with extreme rapidity.

It is therefore with considerable hesitation that an expensive course of vaccine treatment should be suggested to any case of acute infection of the antrum until opportunity for spontaneous cure has been afforded and aided by attention to intra-nasal abnormalities, the institution of facilities for proper drainage and the application of lavage and other usual remedial measures.

Here I would like to say that if artificial drainage has to be established, and the possibility of future vaccine treatment has to 
be considered, then an intra-nasal operation will be a better procedure than puncture through a tooth-socket, for this latter affords unlimited opportunity for the continual ingress of contaminating organisms from the mouth, organisms which may prove especially refractory to vaccine treatment. As soon, however, as an acute infection shows a tendency to assume a chronic state resort should be made to vaccine therapy for the following reasons: (r) Extension to neighbouring cavities may be obviated; (2) truly chronic infections prove decidedly refractory to specific treatment. In by far the greater proportion of the thirty cases which I have seen during the past three years operative measures had been taken, and lavage persisted in for several years. In none of these have I succeeded, even after two years' treatment, in producing such complete cure that vaccine treatment could be altogether discontinued. What I have achieved has been as follows: (I) Great diminution of the secretion, perhaps to such a degree that the performance of lavage once every two or three days by the patient himself has sufficed to maintain a practically complete absence of pus formation; (2) total disappearance of exacerbations and of recurrent attacks of acute nasal catarrh; (3) considerable improvement in the general health. The best results I have obtained have been in two very chronic cases, one of infection by the bacillus of Friedlander, the other by the B. coli. In each of these operative interference was refused, and could not be insisted on, yet the final result was almost complete cure; a short course of vaccine treatment, has, however, had to be continued at fourto six-monthly intervals.

If any measure of success is to be achieved in these very chronic cases it must be remembered (I) that very high ultimate dosages indeed may be requisite, such as 2000 , or even 5000 million B. influenze, I0002000 million pneumococcus, 1000 million streptococcus or $M$. catarthalis, 1000-2000 B. of Friedlander, B. coli or B. protens, 2000-4000 million staphylococcus. The blood supply, especially to the antrum, frontal and sphenoidal sinuses is small, hence the amount of immune bodies carried there is small in any given blood volume; as there is difficulty in increasing the latter it is necessary greatly to augment the former.

(2) That treatment may have to be prolonged, and should be recontinued after intervals, say, of every six months.

(3) That in cases of multiple sinusitis the bacterial flora of the several cavities may differ, and that great care is requisite in making a correct bacteriological diagnosis, and in checking the progress of the immunisation.

(4) That re-infection or fresh infection by other bacteria may at any time occur; inasmuch as the most likely new invaders are the other 
catarrhal organisms a wise procedure is to anticipate the possibility, as far as possible, by the administration, at six-monthly intervals, of three progressive doses of the combined vaccine for colds of the Wimpole Institute.

(5) That when large dosages are being employed the intervals must not be unduly short; ten days or slightly longer usually proves a satisfactory one.

(6) That if progress is interrupted fresh infection is a most likely cause, and is to be determined by careful reinvestigation of the bacterial flora. 


\section{CHAPTER VII.}

\section{VACCINES IN BRONCHITIS AND ASTHMA.}

(a) Bronchitis.-This condition is one pre-eminently suited for vaccine treatment. The diagnosis of the offending organisms presents few difficulties; a suitable vaccine and therefore the production of the necessary immune bodies can be obtained with reasonable certainty; the copious blood-supply ensures the carriage of these latter to the focus of disease, and, as I shall show, there are definite methods of estimating the progress made and so of controlling intervals and dosage. Reference to Table X, p. 53, will show that five organisms, viz. the $B$. influenza, pneumococcus, streptococcus, M. catarrhalis and $M$. paratetragenus are chiefly concerned in the production of this condition; they may occur singly - a rare event-or in any of the varying combinations; usually two or three varieties occur together. While in many cases involvement of the bronchi and bronchioles is sequent to acute catarrh of the upper respiratory tract, yet in certain cases, especially in the old and infirm, and in those specially prone to bronchial infections, the involvement of the lower passages would appear to be the primary one; in these instances the infection is the more likely to be a simple one. Streptococci are of considerably more importance in catarrhs of the lower respiratory tract than of the upper, and may belong either to the so-called "salivarius" type, or to the varieties depicted in figs. 7 and Io, Plate III; these latter when present would appear to be of special importance.

It is fairly obvious that autogenous vaccines are more likely to be efficacious in the treatment of this complaint than are stock ones, and it is especially important to bear in mind that variation in the flora is very liable to occur during the progress of immunisation, and that a blend of vaccine admirably adapted for treatment at the beginning may be totally unsuited to the later stages; if this be carefully remembered, much disappointment, both to immuniser and patient, will be avoided. In early stages of the acute bronchitic attack bacteria may be few, the sputum being composed chiefly of hyalin mucus containing bronchial 
cells in a state of rapid degeneration; at the height of the attack mononuclear pulmonary cells make their appearance, indicative of extension of the process to the terminal bronchioles and even the alveoli; during subsidence the hyaline mucus becomes replaced by large mucous networks, containing within the meshes many bacteria and polymorphonuclear leucocytes. The examination of smears suitably stained with Unna's polychrome blue or in default of this with Leishman's stain thus affords a fairly reliable guide as to the stage and progress of the infection. The daily measurement of the sputum and its naked-eye appearance, the pulse and temperature also prove of service in estimating the patient's progress. The most accurate method of controlling intervals and dosage, one, moreover, applicable to all infections of the lower respiratory tract, is that based upon careful stethoscopic observation. The procedure depends upon the following facts: (I) that any dose of vaccine which does not produce a definite reaction at the focus of disease is either inadequate or not compounded of the appropriate immunising agents.

(2) That such a dose as causes definite improvement in the clinical signs within twelve hours is affording but a minimal stimulus, and may probably be increased with some advantage.

(3) That such a dose as causes definite extension of the signs within twelve to eighteen hours, the appearance of signs where none previously existed, or an increase in their volume or moistness is indicative of correct dosage provided that rapid improvement in each of these particulars ensues within the next few hours.

The patient having been placed under the most favourable conditions, attention having been paid to such general forms of treatment as experience indicates, and the vaccine having been prepared, systematic stethoscopic examination of the chest is then performed, and the clinical signs carefully noted upon a suitable chart; the chest charts usually employed are not well adapted for the purpose, the outlines of the ribs occupying and obscuring too much of the space; a much better form has been prepared for me by H. K. Lewis, and may be seen in Charts V-XI; ample room is afforded for the insertion of such signs as we may employ, those indicated on the margin being convenient, and affording at a glance such information as may be desired.

The selected dose of vaccine is then administered and fresh observations taken at the end of twelve and tiventy-four hours and daily thereafter. Assuming that a definite reaction has been first produced, steady improvement in the condition is indication of withholding any fresh inoculation, at all events for seven or eight days, unless retrogression occurs, especially if this be accentuated upon 
observation twenty-four hours later, when the necessity of reinoculation is thereby indicated. Increase of dosage is necessary when improvement is maintained for only three or four days, or in default of adequate response.

\section{Front of Chest}

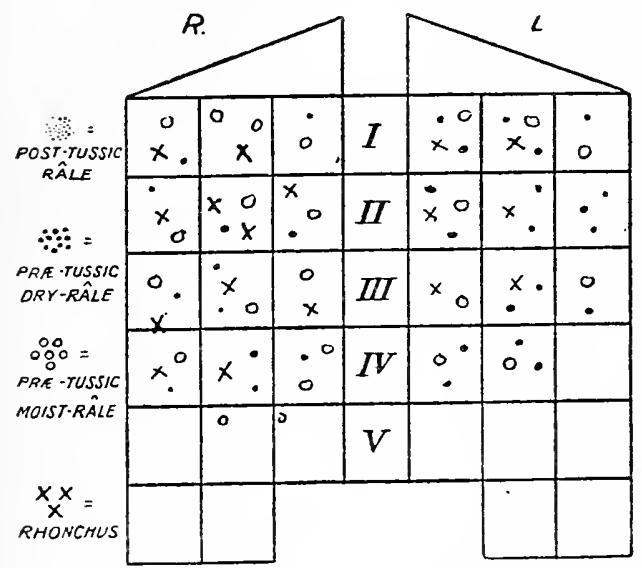

Chart V.-Before first inoculation.

\section{Back of Chest}

\begin{tabular}{|c|c|c|c|c|c|c|}
\hline 0 & $x$. & ${ }^{0} x$ & $I$ & $x_{0}$. & $\begin{array}{l}0 \\
x\end{array}$ & \\
\hline : & $x_{0} \cdot$ & $x_{0}^{0}$ & $\Pi$ & $\therefore 0$ & $x^{\circ} 0$ & $x$ \\
\hline$x:$ & $0^{x}$. & $x$ & $W I$ & $0_{0}^{x}$. & $x_{0}$. & D \\
\hline$\because 0$ & $\cdot \dot{x}$ & $\circ$ & $I V$ & 0 & $x_{0}$ & 0 \\
\hline . & . & - & $V$ & $\because 0$ & - & \\
\hline . & $x^{\cdot}$ & $o$ & $V I$ & & & \\
\hline & & & $V W$ & & & \\
\hline
\end{tabular}

Sputum $=6 \mathrm{oz}$. per day.

Back of chest

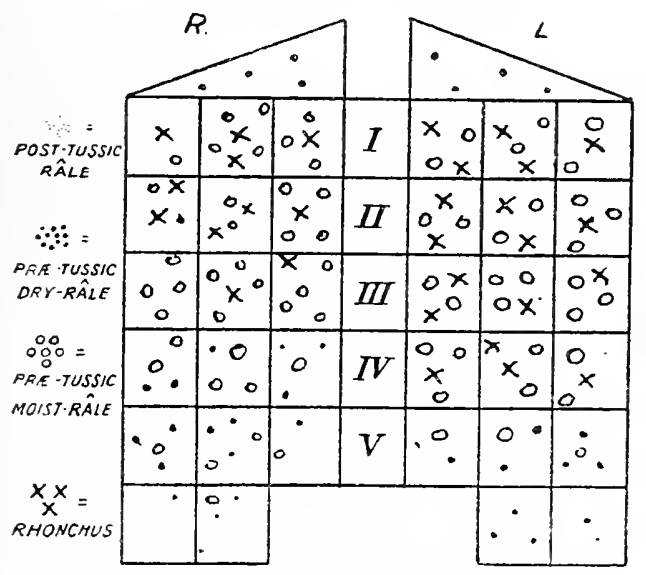

\begin{tabular}{|c|c|c|c|c|c|c|}
\hline$x^{0}$ & $\begin{array}{ll}x & 0 \\
0 & x\end{array}$ & $\begin{array}{l}x_{0} \\
x^{0}\end{array}$ & $I$ & $\begin{array}{r}0 \\
\times 0\end{array}$ & $x_{0}^{0}$ & \\
\hline$\stackrel{0}{x}_{0}$ & $\begin{array}{c}\times 0 \\
x\end{array}$ & $\begin{array}{ll}0 & x \\
x & 0\end{array}$ & $\Pi$ & $0 x_{0}^{0}$ & $x_{0}^{0}$ & $0 x$. \\
\hline $\begin{array}{r}0 \\
x_{0}\end{array}$ & $\begin{array}{l}x \\
0 \\
0_{x}\end{array}$ & $\begin{array}{l}0 \\
x_{0}\end{array}$ & $I I I$ & ${ }_{0}^{x} \circ$ & $\begin{array}{l}0 \\
\times 0\end{array}$ & $\dot{0}_{0}$ \\
\hline$\times_{0}^{0}$ & $x^{\circ}$ & $x^{0}$ & $I V$ & $x_{0}^{0}$ & ${ }_{0}^{0}{ }_{0}^{0}$ & $\therefore$ \\
\hline$\because 0$ & $x$ & $x^{0}$ & $V$ & $\begin{array}{cc}0 & x \\
0\end{array}$ & - $: 0$ & $\circ$ \\
\hline \multirow[t]{2}{*}{ ? } & $x^{0}$ & $\begin{array}{ll} & 0 \\
0 & \\
\end{array}$ & $V I$ & 0.0 & $\because 0$ & $\therefore$ \\
\hline & ${ }^{0}$ & • & $\sqrt{I I}$ & & & - \\
\hline
\end{tabular}

Chart VI.-Twelve hours after first inoculation. Sputum = Io oz. per day.

Charts V-XI will serve to elucidate my meaning, and show admirably the progress made by a certain severe case of acute recurrent bronchitis in an aged and not very hopeful case.

The patient had been affected for at least ten years by chronic bronchitis following upon an attack of apparently true influenza-three or four times during each of the last three years there had been acute 
exacerbations; the attack for which I was consulted had lasted about a fortnight, respiration was very much embarrassed, little relief was afforded by the various stock remedies, and sleep was very difficult to procure. When I saw him the patient looked very ill indeed, but two

Front of Chest

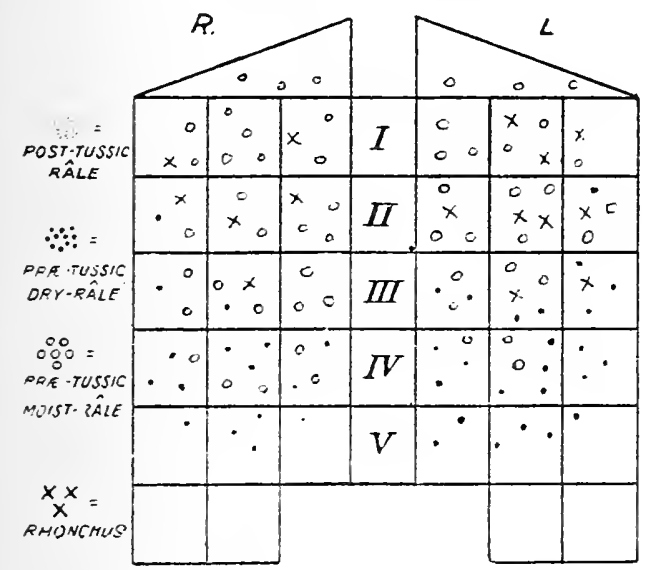

Back of Chest

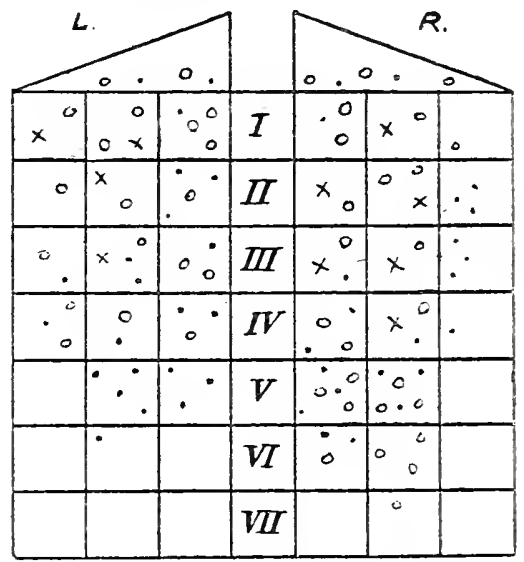

CHART VII.-Twenty-fours hours after first inoculation. Sputum $=6$ oz. per day.

Front of Chest

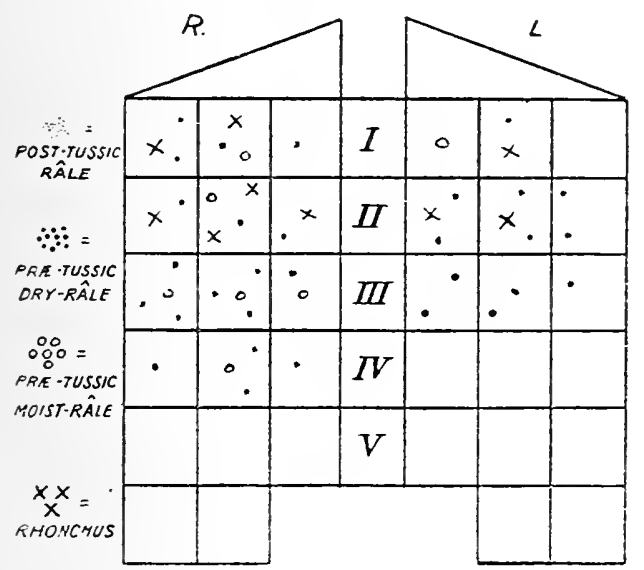

Back of Chest

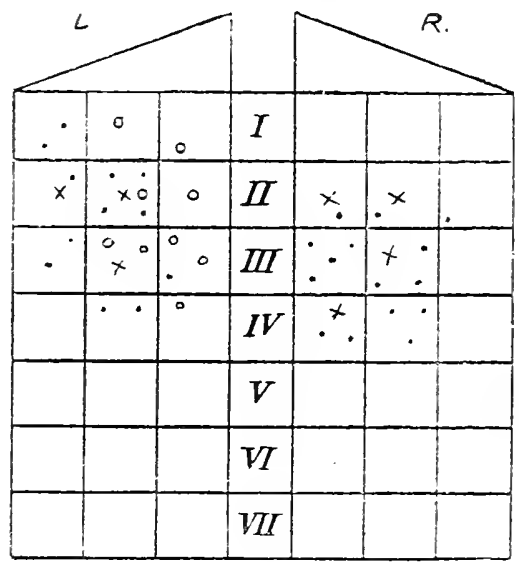

Chart VIII.-Seventy-two hours after first inoculation. Sputum $=2 \mathrm{oz}$. per day.

good features were-(I) the pulse was regular and of good volume; (2) the tissues were firm and apparently healthy despite the ill look of his face. A bacteriological examination showed that the sputum was swarming with $B$. influenza, and contained also many pneumococci and M. catarrhalis. An autogenous vaccine was prepared of each of these, and treatment begun with a dose of 50 million $B$. influenza and 25 
million each pneumococcus and $M$. catarrhalis. As will be seen from the charts a marked local reaction was produced within twelve hours, the physical signs being increased considerably, as was also the amount of sputum; of constitutional symptoms there were none beyond slight
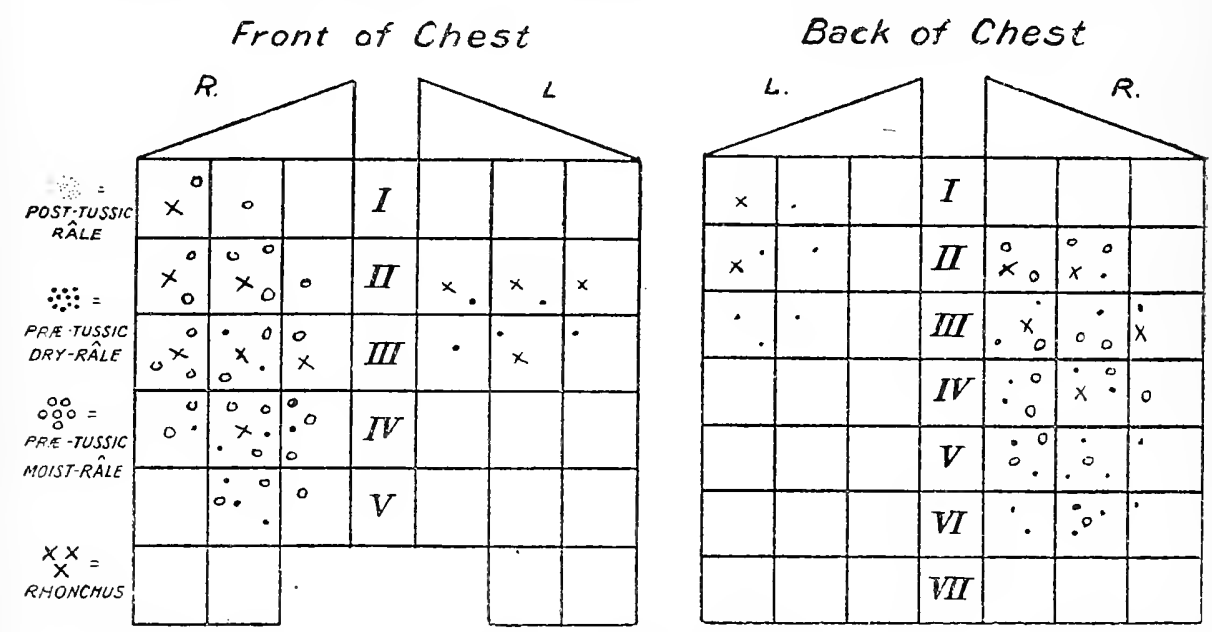

Chart IX.-Six days after first inoculation. Second inoculation. Sputum $=3$ oz. per day.

Front of Chest

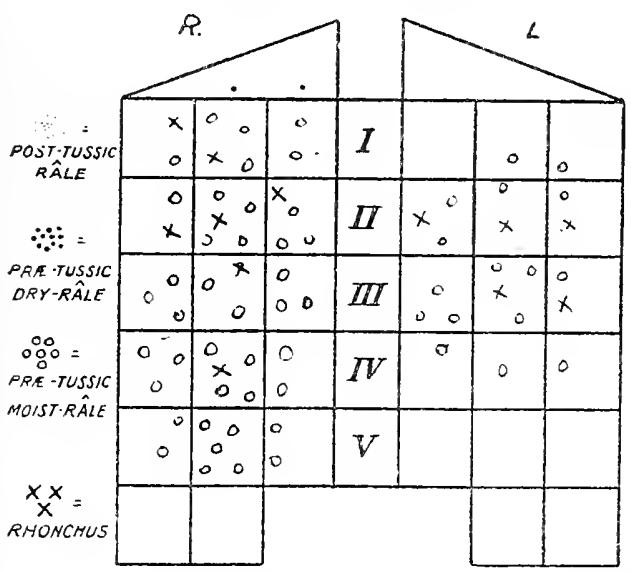

Back of Chest

\begin{tabular}{|c|c|c|c|c|c|c|}
\hline$x_{0}$ & $\begin{array}{l}x \\
0\end{array}$ & c & $I$ & & 0 & \\
\hline${ }^{x}{ }^{0}$ & $0^{x}$ & 0 & $n$ & $0_{0}^{0} x$ & $x^{0}$ & \\
\hline \multirow[t]{5}{*}{$x$} & $0^{x}$ & 0 & $W I$ & $\begin{array}{ll}0 \\
0\end{array}$ & $0^{0} 0$ & : \\
\hline & 0 & & $I V$ & $0_{0}^{0}$ & $\times \stackrel{0}{0}$ & 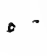 \\
\hline & & & $V$ & 00 & $\begin{array}{ll}0 & \\
0 & 0\end{array}$ & 0 \\
\hline & & & $V I$ & $\begin{array}{c}0 \\
0\end{array}$ & $\begin{array}{cc}0 & 0 \\
0\end{array}$ & 0 \\
\hline & & & $V E$ & $c$ & 0 & 0 \\
\hline
\end{tabular}

Chart $\mathrm{X}$.- Twelve hours after second inoculation. Sputum $=4 \mathrm{oz}$. per day.

increase of pulse-rate. At the end of twenty-four hours improvement began to set in, and the patient passed the best night for a fortnight; the improvement continued markedly during the next three days, then there was a slight relapse. Accordingly on the completion of the sixth day the initial dose was repeated, again with such highly beneficial results that at the end of the eighth day after beginning the vaccine treatment 
the patient was sleeping well, only being awakened by one or two slight fits of coughing, was able to recline in comfort, was eating well, and expectorated only $\frac{1}{2} \mathrm{oz}$. of sputum in the twenty-four hours. Convalescence was uninterrupted, and the patient, instead of leaving for Madeira, as had been intended, was able to winter on the south coast of England. Inoculations were continued at intervals of six to eight days, and about two months after the beginning of treatment examination of the sputum showed a reduction in the organisms by many hundredfold; the same varieties, however, were still present, and expectoration persisted to the extent of $2-3$ drachms per day. At this time a dosage of 250 million $B$. influenza and 100 million $M$. catarthalis and pneumococcus was being employed. I therefore advised the use

Front of Chest

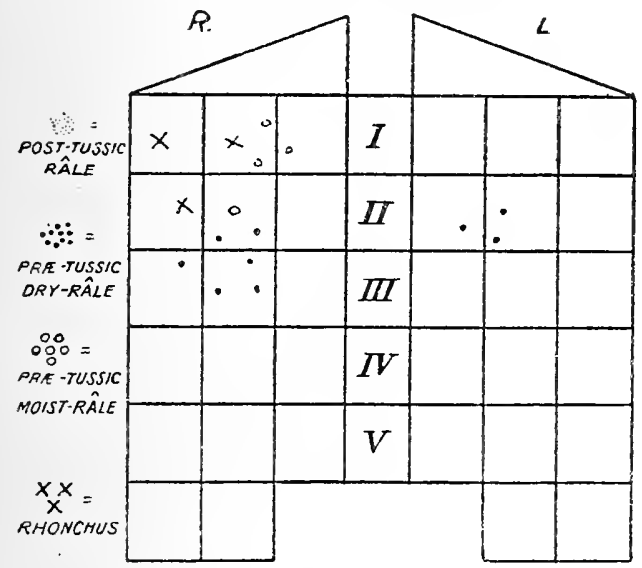

Back of Chest

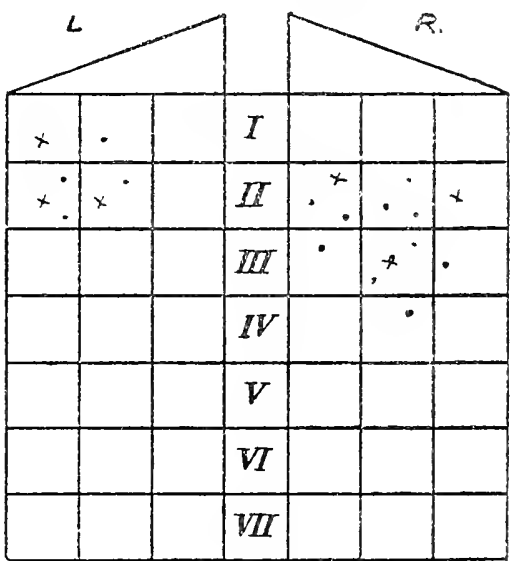

Chart XI.-Forty-eight hours after second inoculation. Sputum $=\frac{1}{2}$ oz. per day.

of double this dosage at intervals of eight days. At the end of another two months further slight reduction in the amount of sputum had been attained, and examination showed that the B. influenze had almost if not entirely disappeared (none at all could be cultured); the pneumococci had also gone, but the $M$. catarrlalis had somewhat increased in actual numbers. A fresh vaccine was therefore prepared, and treatment continued with dosages of 250 millions at eight-day intervals. The patient is now practically well, merely expectorating one or two nummular masses of sputum on waking; these contain nothing but a few $M$. catarrhalis. With this condition the patient is well satisfied, and it merely remains to forestall fresh infections by the use of two or three immunising doses about every four months, suitable administrations being the following sequence at eight- to ten-day intervals: (I) $B$. influenza roo millions, pneumococcus 50 millions, $M$. 
catarrhalis roo millions; (2) B. influenza 250 millions, pneumococcus roo millions, $M$. catarrhalis 250 millions; (3) B. influenza 500 millions, pneumococcus 250 millions, $M$. catarrialis 500 millions. Should, despite this, a fresh attack occur, the first essential will be a re-examination of the sputum in case infection has been set up by some other organism. If, however, one of the old ones shall have again come to the front, the determination of suitable dosage will depend upon the interval that has elapsed since the last immunising dose; if this has been recent, say within a month, then treatment will be begun with the second of the above three dosages; if on the contrary it is near the end of the four months' interval, then with the first of these; but rapid advance in dosage will probably prove advisable (this, however, will be determined as before by stethoscopic and other observations).

I could relate the histories of many cases similar to the above, but this would prove of little advantage; for inasmuch as the careful clinician will find no difficulty whatever in understanding the rationale of the above procedure, and so of conducting such a case for himself, it only remains for him to remember that the other essentials to a successful issue are-

(I) Accuracy in diagnosis of the infecting organism or organisms.

(2) Careful preparation of the appropriate vaccine.

(3) Repeated checking of the progress of immunisation by means of bacteriological examinations, which will at once indicate whether change of vaccine is necessary.

(4) No undue haste in discontinuing treatment; for so long as the pneumococcus or B. influenza lurks in the lung, in no matter how scanty numbers, it is always a source of continual and considerable potential danger.

(5) The application of all other such adjuvants as clinical experience has proved of value.

Old age and a desperate condition of the patient are no contraindications to the application of vaccine treatment, but quite the contrary; they merely indicate extra care in the choice of suitable dosages, which should err rather on the side of under-dosage than on that of over-dosage; the clinical examination of the chest will afford unerring indication as to whether immunising responses are being made or not; in the latter event dosages must be pushed and intervals between them shortened, to three, or even two days if necessary, until an immunising response is obtained; when this has been achieved subsequent increases should be made with care and discretion, and intervals lengthened if so indicated by the clinical observations.

In conclusion, I would merely add that I have never seen the case which did not benefit immediately and markedly from the administration 
of the suitable vaccine in suitable dosages at suitable intervals, and that I do not believe such a case exists; in no other bacterial infection of any part of the body are such gratifying and striking results to be secured; even capillary bronchitis I do not regard as beyond the reach of this form of treatment.

(b) Asthmna.- When we consider the case of asthma we find that we are dealing with an affection of quite a different kind. Bronchitis is a bacterial disease and nothing else; whereas, were the results of vaccine treatment in asthmatic conditions not available, it would be impossible to assert with any confidence that asthma is ever due to a bacterial infection. The fact, moreover, that vaccine treatment does in a certain percentage of cases prevent the onset of dyspnœic attacks-and this it most certainly does-fails to establish it as a fact that the bacteria are the true cause of the asthma; it may only mean-and this I believe to be the case-that a bacterial irritant may suffice to set a delicately poised already existent mechanism in action-the clock may be wound up, it only requires a touch of the pendulum to set the works in motion; this, in certain instances, bacteria or their toxins suffice to do. It thus follows in some cases, where due attention has been paid $(a)$ to the true ætiological factor of the asthmatic spasm. (b) to other accessory factors, such as diet, condition of the borvels, place of residence, etc., and where these procedures have not sufficed to cure the patient, that benefit will accrue from therapeutic immunisation. That this would be the more likely to occur in cases where bronchitic symptoms are marked, expectoration copious, and the bacterial flora profuse, might be anticipated, but within my experience this is not necessarily so; the best results are oft obtained in cases characterised by the scanty, viscid, stringy mucus of true asthma, containing, relatively to a bronchitic sputum, but few bacteria in but few varieties.

Although I fear to become tedious by undue repetitions, here more than in any other disease with which bacteria are associated is it essential to ensure that proper treatment be first directed to all abnormalities, errors of respiration and of diet. When this has been done and the usual methods for securing cure or alleviation have failed, resort may then be made to vaccine treatment, and some hope of amelioration, if nothing more, may be offered to the patient.

Reference to Table XI, p. 54, will show that the organisms most commonly found in the sputum of asthma are the streptococcus, $M$. catarrhalis and pneumococcus.

The streptococcus, which may be brevis, longus, or maximus in type, is present in no less than $9^{6}$ per cent. of all cases, and within my experience in Ioo per cent. of those in which bronchitis symptoms are in abey- 
ance; the $M$. catarrhalis, on the other hand, is the more common in those cases in which bronchitis is a marked feature; this also holds true for the B. influenze, $M$. paratetragenus and pneumococcus. As I have already said, considerable complexity in the bacteriological findings is uncommon; binorganismal infection, and especially by a streptococcus and the M. catarrhalis, is that most frequently found.

The nature of the infection is not without bearing upon the prognosis of the vaccine treatment; if the Streptococcus longus or maximus be present, amelioration of the asthmatic condition under judicious treatment is a practical certainty; if the $M$. catarrhalis predominates bronchitic catarrh is sure to be marked, and it is no uncommon result to find that as the amount of the bronchial secretion is reduced, the difficulty in expulsion of the more tenacious and more truly asthmatic sputum becomes increasingly greater and the tendency to spasms more pronounced; this sometimes indicates that streptococci have come more to the front, and that a change of vaccine is probably advisable, but even if this be done it by no means follows that a favourable result will be achieved, and personally I regard the steering of a case of asthma to the desired haven as difficult a manœuvre as it is easy in a case of bronchitis.

There are other factors, too, which prove most difficult to control. A case of streptococcal asthma may be making all the progress one could desire, when unhappily a fresh bronchial infection by the pneumococcus or more especially the B. influenza suddenly occurs. Not only may this prove difficult to eradicate, but the unfortunate result is also brought about of a resultant unstabilising of the already none too stable centre of control for the blood-supply to the lung. Dr. Alexander Francis tells me that he has frequently noticed this effect produced by infection by the $B$. influenze in cases wherein he has stabilised the vaso-motor centre by means of cauterisation of the nasal mucosa, and that he, too, has found it an influence peculiarly difficult to overcome.

Lest, however, it be thought that the vaccine therapy of asthma is so fraught with difficulty and disappointment as to be nothing worth, let me here remark that in a certain percentage of cases the results have been so good that the patients utterly refuse to discontinue the occasional use of their vaccines, but every six months or so go through a short course of immunisation.

The initial dose of the various vaccines which I usually employ are as follows: B. influenza Ioo millions, $M$. catarrtalis, $M$. paratetragenus, and pneumococcus 50 millions, streptococcus 25 millions, and whether the vaccine is likely to prove of service or not will be almost certainly determined by the response to this dose or to one of double magnitude. 
During the night of the inoculation the patient will be awakened by an asthmatic attack, whether he be usually subject to one or not; in the former case the attack may be a bad one and necessitate control either by the subcutaneous injection of $2-3$ minims of adrenalin solution $\mathrm{I}$ in rooo, or by whatever means is usually found to prove efficacious; in the latter case the attack will be a mild one and pass off within an hour or two. If the patient be likely to benefit by the treatment and such a reaction be obtained, during the ensuing five or six days there should be increased immunity from attacks both in number and severity; tendency to relapse is the sign for re-inoculation; failure to respond by the production of a slight attack the sign for increased dosage. Occasionally, it is true, the patient does not react to the dosage employed by the production of an attack within twelve hours, but by a decided lessening in the number and severity of attacks; this indica. tion of resulting immunity is one to which the patient will take no exception.

My observations upon the vaccine treatment of asthma have by no means yet reached finality, and it is therefore impossible to be very dogmatic upon any point, whether of bacteriology, course of treatment or results; a short history of two cases will serve, perhaps, to illustrate some few points which I have endeavoured, perhaps, with ill-success to make clear.

CASE I.-Mrs. A-, aged 23 years, had suffered from severe asthma since operation for adenoids and enlarged turbinate bones two years ago; paroxysms very severe, lasting several hours, and for the past few months of daily occurrence. As she was perfectly clear in her own mind that the asthma was due to the nasal operation, she evinced a very strong objection to the performance of cautery of the mucosa, and wished first to try vaccine treatment.

A bacteriological examination showed streptococcus, both longus and brevis, and M.catarrhalis. An autogenous vaccine was prepared.

On October I2th inoculation of Io million each streptococcus and 25 million $M$. catarrialis.

On October 2oth she presented herself, looking much better and less jaded, and reported that she had had an attack on the second and subsequent nights, with the exception of the last two, when she had been perfectly free. The dosage was increased by one half.

October 27 th : She reported that on the night of the inoculation and that following she had had attacks of moderate severity, but since then had been quite free from any but very mild daily attacks. Repeated last dosage.

November 7 th : Reports a bad attack on night of October 27th; since then only very mild daily attacks. Repeated dosage. 
November $5_{5}$ th : Continued improvement in general health; no pronounced reaction after last; very slight daily attack. Repeated dosage.

November 27 th : Reports a bad attack on night of 23 rd due probably to indigestion, otherwise only mild daily attack. Repeated dosage.

December 6th : Had a bad attack on night of November 28th; no others by day or night till morning of December 6th. Repeated dosage.

December I5th: A very slight attack on night of December 9th; none whatever since; double initial dosage given.

With the result so far the patient was delighted and I more than pleased; in the last week of December she caught the prevailing influenza epidemic, swarms of the bacilli being found in her sputum along with some pneumococci. She was quite prostrated, and as the asthmatic attacks soon recurred with all their old violence, recourse had to be had to adrenalin subcutaneously; this controlled the severity of the attacks admirably, but seemed to have no influence upon their frequency. A fresh vaccine of $B$. influenza and pneumococcus was prepared to combine with the streptococcal-catarrhalis one. Doses of the combined vaccines were given on January 8th, 17 th, 27 th, February 6th and Igth, but the infection by the B. influenza proved very hard to eradicate; even when this was apparently done the frequency and severity of the asthmatic attacks, which now refused to be controlled by the original vaccines, were such that I no longer found difficulty in persuading the patient to place herself in Dr. Alexander Francis's hands; she now informs me that the result of the cauterisation has been entirely satisfactory.

The unhappy effect of infection by the $B$. influenze is only too well shown in this case.

CASE 2.-Mr. B-, aged 50 years, outdoor telegraph superintendent in the north of Scotland. Weight formerly Io st. $6 \mathrm{lb}$., now $8 \mathrm{st} .6 \mathrm{lb}$.; complains of chronic bronchitis with spasmodic asthma.

History was as follows :

January, I909: Acute attack of bronchitis, lasting for six weeks, then recovery.

January, I9I0: Another acute attack lasting eight weeks, and leaving behind it considerable dyspnœe, which, by June, IgIo, had assumed a spasmodic form, and become so severe that he was incapable of any exertion. On September $I 7$ th he suddenly recovered, and remained quite well till January 3rd, IgII, when a fresh acute attack of bronchitis supervened; this persisted till the end of March, by which time partial recovery was established; regular attacks of spasmodic dyspnœa, however, continued, and in September, I9II, he had â bacteriological examination made of his sputum, and a vaccine containing 20 million M. catarrhalis and Io million pneumococcus was 


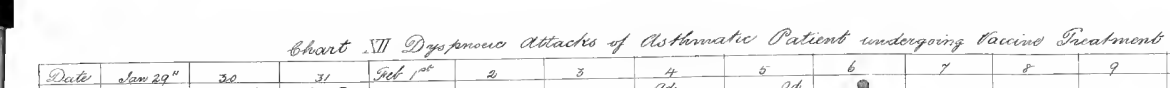

a.
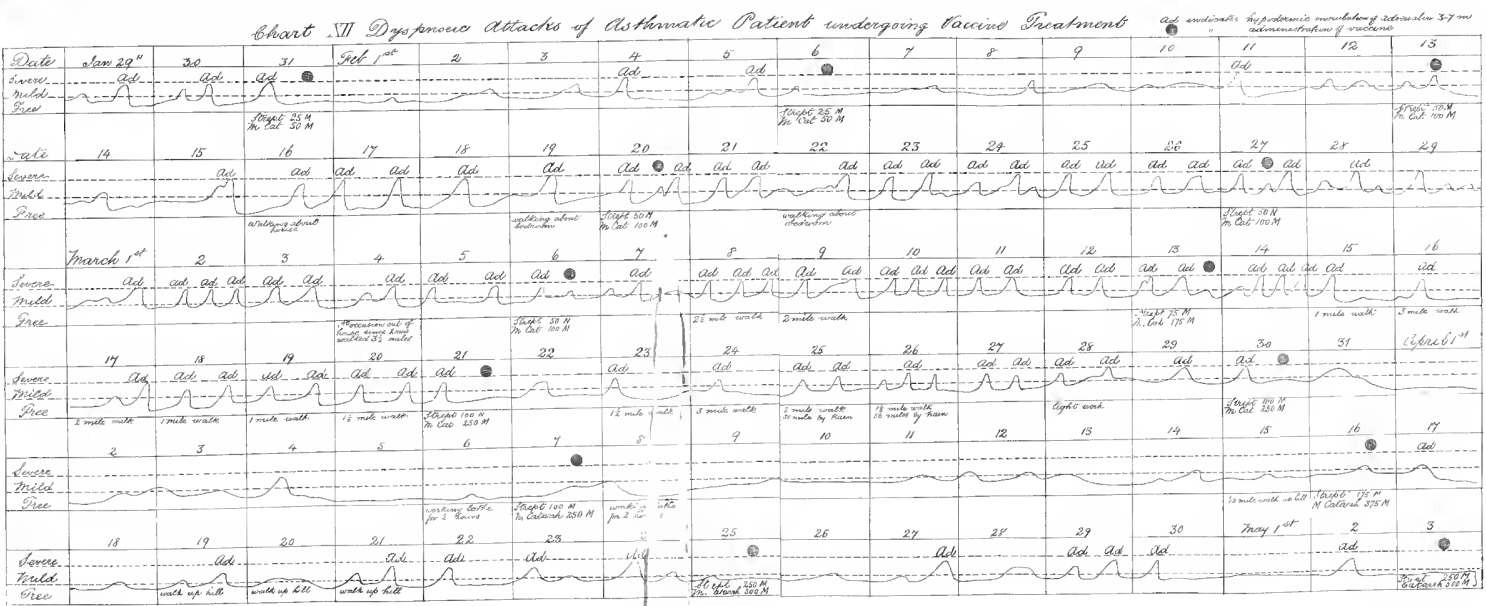

prepared, but not by me, and administered at intervals of two to four days. Slight immediate improvement in the direction of lengthening of the periods of freedom from dyspnœa was produced.

September, IgII, to January, IgI2: Patient states that the dyspnœic attacks continued with great regularity at an average interval of nine hours. Minimum interval, four hours; maximum, twenty hours. The only remedy which gave any certain relief was adrenalin chloride subcutaneously.

At the end of January, I9I2, he consulted me by post as he had long been unable to leave the house. I examined the spintum, and found Streptococcus maximus in considerable numbers, and a few $M$. catarrhalis and pneumococcus. Informed him that he had hardly given the previous vaccine a fair trial, but that I thought I could compound one rather better suited to his needs if he would place its administration in the hands of a medical man, who would allow me to direct treatment. Dr. K. Gillies kindly undertook the task, and began treatment with an initial dosage of Streptococcus maximus 25 millions, pneumococcus 25 millions, and M. catarrhalis 50 millions.

On March 29th, I9I2, I received a report from the patient and make the following abstracts: "Prior to commencing with your vaccine I had very short periods of freedom from dyspnœea, and that with entire rest and confinement to my bed-room. Now you will observe I have much longer periods of ease and that with more movement about. These have, as is shown on the chart, been temporarily affected by any labour, stress, etc. That I have been able to get out at all this cold weather shows a very marked improvement. My appetite is good considering the little exercise, my flesh is clear and more ruddy, and my weight has increased $6 \mathrm{lbs}$. during the last eight weeks. My sputum is becoming very scanty; you asked me to send a fresh specimen if I found my progress not satisfactory. I do not think the time has arrived for this as I consider I am so very much improved." On May 7 th I received a fresh report: from May ist to to Nay 5 th inclusive he had only experienced one moderate dyspncic attack, which had at once yielded to a small dose of adrenalin chloride. By this time Dr. Gillies had increased the dosage of vaccine to ten times the initial one, and doses of even this magnitude failed to excite a dyspnœic attack. A rough idea of the progress of the case may be gathered from Chart XII.

In order to arrive at a just appreciation of the result it must be remembered, as the patient says, that after a long period of complete confinement to his room, he was at the time of the report indulging in considerable out-door exercise at a most trying period of year to all asthmatics. 
In conclusion I would merely say that although many of my cases have pursued more favourable courses than these two, it is yet impossible for me to dogmatise at all; it must suffice to state that within my experience much good may accrue to asthmatic cases by a course of vaccine treatment, but that with this disease more than with any other each case must be considered apart and treated on its own merits, not according to any given procedure or rule of thumb. 


\section{CHAPTER VIII.}

\section{VACCINES IN PNELMONIA.}

Defnition.-Pneumonia typically is an acute febrile disease, beginning as a rule suddenly but sometimes insidiously, associated with massive consolidation of the lung, running a fairly definite course, and terminating within ten days by crisis or lysis. Inasmuch, however, as the course is sometimes less definite, the term is taken to include all cases of acute febrile disease accompanied by massive consolidation of the lung.

Etiology. - While it is undoubtedly true that pneumonia is a disease characterised especially by sudden onset, I think that in a considerable proportion of cases of true lobar pneumonia it would be more accurately regarded as an acute exacerbation in a chronic infection by the pneumococcus of the bronchial or pulmonary tissues, an infection which originally took its rise in an acute pneumococcal infection of the upper respiratory passages, and subsequently invaded those of the chest, giving rise, perhaps, to nothing more than a bronchial catarrh, which apparently cleared up more or less completely. As I have already mentioned previously I have had the opportunity of watching several such cases, some for several years, and of making periodic examinations of the sputum, which may be extremely scanty, and of noting with what extreme tenacity the pneumococci retain their hold upon the tissues. In some instances the most patient treatment by means of vaccines and otherwise fails to dislodge them completely.

On page I93 of No. 7, vol. i, of the Journal of Vaccine Therapy', Dr. Graham Morris tells his own story, which illustrates well this tendency of the pneumococcus to persist in the pulmonary tissues.

I do not for a moment mean that all attacks of acute pneumonia are due to a lighting up into virulence of pneumococci long dormant in the pulmonary tissues; in some instances an acute nasal or tracheal catarrh rapidly tracks down into the chest, especially when the $B$. influenzed is associated with the pneumococcus, and sets up an attack 
of acute pneumonia in those who apparently have not suffered for many years from any catarrhal affection of the chest; while yet again, and in perhaps the majority of cases, the pneumonic attack is not preceded by any obvious catarrh of the upper respiratory passages. This is entirely a question of local immunity. I have just seen a patient from Guatemala, who tells me that every year there is a pneumonia epidemic of exceptional virulence; he himself and others of his acquaintance have never fallen victim, but are affected by very severe recurrent catarrhs of the upper passages; in his case these are entirely confined to the nose and post-nasal space. From these parts I have taken cultures and found thousands of pneumococci in each loopful of mucus; with him the resistance of the pulmonary tissues is obviously very high, whereas with many hundreds of the natives it is correspondingly low.

Of this, however, I am absolutely convinced, that in the United Kingdom the percentage of cases in which the pneumococcus persists in the lung after merely causing an acute bronchial catarrh is very much greater than is commonly imagined, and that their presence there is fraught with danger to the host. The consideration of this question will be resumed later.

Bacteriology:-Until recently the pneumococcus of Frankel was regarded as the specific and only cause of acute pneumonia; it is now known that other bacteria are capable of setting up this condition, either alone or in combination with each other or associated with the pneumococcus; in the last instance doubt is cast upon their ætiological significance. It is otherwise when they occur alone, and it must be granted that the following may be responsible: Friedlander's pneumobacillus, B. influenza, Streptococcus mucosus, staphylococcus, B. typhosus, B. coli. Some of these I propose to mention a little in detail.

"Friedlander" pnenmonia was first described by Philippi (Munch. med. Woch., I902), later by Lenhartz (Nothnagel's Spezielle Path. und Therapie, Bd. iii), by Apelt (Munch. med. Woch., I908, p. 833), and by others, while Stühlern (Zentral. fïr Bakt., Bd. xxxvi, I904) carefully studied forty-five cases, and Kokawa (Deut. Archiv fur klin. Med., I904) eighteen cases, and Cordier Badolle and Brissaud (Lyon Med., April Ifth, I9I2) have recently reviewed the whole subject of Friedlander pneumonia. These authorities all agree that there is a distinct form of pneumonia due to this bacillus, characterised by the great formation of mucus in the infiltrated areas, by the surface of the lung being sometimes covered with slimy exudate, by the imperfect development of "red hepatisation," this having a grey to black appearance, by the absence of marked fibrinous exudate and of hæmorrhage. On the other hand there is a great tendency to massive blood infection and to 
the disease running a malignant course: while suppuration and abscess formation are especially liable to occur. The distribution may be either lobar or lobular.

Streptococcus pneumonia appears to be almost indistinguishable from the pneumococcal variety, beginning, like it, with a rigor or pleuritic pain, and being associated with herpes labialis. The pyrexia, however, tends to be intermittent, and may persist for a considerable time, while the physical signs may be those of broncho-pneumonia. Filarelow has described four such cases, and Schottmuller (.Yunch. med. Woch., Igo3, p. I 427$)$ six in which it was lobar in type.

Typhoid pnemmonia is regarded by some authorities as nothing else than a pneumococcal pneumonia occurring during the course of typhoid fever, in which the presence of the pneumococcus is orershadowed by the $B$. typhosus, the latter being nothing more than a secondary invader. With the statement that the pneumococcus is always present in such cases I am not prepared-perhaps on insufficient grounds-to agree. In a case of typhoid pneumonia which I have recorded (Vaccine Therapy, Edit. 3, p. I 5 ) the bacteriological findings were B. typhosus and a few Streptococcus longus. The immediate good result produced on the lung condition by an inoculation of an autogenous typhoid vaccine would appear to show that this organism was the important ætiological factor.

Influenza pnemmonia is worthy of much more study than has been accorded to it. The pneumonia superrening on an attack of so-called "influenza," this latter being much more frequently a pure pneumococcal or a combined pneumococcal and influenzal infection, is commonly regarded as a fresh superimposed infection by the pneumococcus; this is an utterly erroneous view in the great majority of cases. On the other hand, a true $B$. influenza infection of the respiratory tract does sometimes culminate in a pneumonia; this pneumonia may be due to a fresh infection by the pneumococcus, but occasionally the infection remains a pure $B$. influenze one. I hare only seen two such cases; the type was broncho-pneumonic, and was characterised by prolonged high pyrexia, great constitutional depression and a malignant course, each case ending as one of lung abscess.

I have entered into this aspect of pneumonia somerhat fully for the following reason. When some years ago I suggested that for immunising purposes a vaccine of the various paratyphoid organisms should be combined with the ordinary typhoid vaccine I was derided by several critics; the careful work that has since been done shows that the proportion of cases diagnosed as typhoid fever which are really ones of paratyphoid fever is in some localities and in some epidemics anything but negligable; in one instance ro per cent. and in another 25 per 
cent. of the cases were found to be in reality paratyphoid fever. In the same way I feel sure that accurate bacteriological examinations would show that the percentage of cases of pneumonia due to bacteria other than the pneumococcus is considerably greater than is supposed. From the purely clinical aspect this may not be of great import; to those who contemplate the routine treatment of pneumonia with vaccines it is a matter of considerable importance.

This leads me to a brief consideration of the methods whereby a correct bacteriological diagnosis may be made. There are three chief ones, viz. (I) sputum examinations, (2) blood examinations, (3) lung puncture observations.

To the essential precautions which must be observed in the collection of sputum for the purposes of a bacteriological examination I have already referred on several occasions. The due observance of these will tend greatly to diminish the difficulties experienced by many observers. While it is true that sputum may be very scanty or even absent in the first day or two of the pneumonic attack, this is not often the case; a very small quantity indeed will suffice, and careful insemination thereof on blood-agar plates will almost invariably lead to the ready isolation of the pneumococci or other organisms, no matter how few these may be. At the same time if no sputum be obtainable or a hasty examination of a stained film leads to anticipation of failure blood culture should be at once resorted to ; Io to 15 c.c. of blood should be withdrawn from a vein of the arm with due precautions; I c.c. should be spread over the surface of an agar plate, the balance introduced into roo c.c. of dextrose peptone broth. The failure of some observers to secure more than 25 per cent. of positive blood-cultures I believe to be due (I) to the employment of too little blood-at least io c.c. should be taken; (2) to the insufficient dilution of the blood with culture fluid--the blood should be diluted eight to ten times; (3) to the use of ordinary broth instead of broth containing 2 per cent. dextrose. Occasionally incubation has to be continued for forty-eight hours, but this is very rarely so. Some observers have obtained positive blood cultures in 80 per cent. of cases, and it would appear that there is in the first few days a true bacteriæmia ; in the case of "Friedlander" and "typhoid" pneumonia vast numbers of the bacteria may be present in the blood.

The procedure for lung puncture I have already described on p. Io.

By a judicious combination of these methods I believe that a reliable diagnosis of the true infective agent may be made in every case without exception. 


\section{The Prophylaxis of Pneumonia.}

If the view I have already enunciated as to the manner in which many cases of acute pneumonia originate be correct, it at once follows that no case of respiratory catarrh due to the pneumococcus, $B$. influenza, streptococcus or B. of Friedlander is to be lightly regarded or to be considered as cured so long as there is any sputum in which the organism can be discovered, or so long as any physical signs persist in the chest. Treatment by means of vaccines must be continued until complete disappearance of the infection is secured; and if this cannot be effected, as will sometimes prore to be the case, then the patient's immunity must be maintained at as high a level as possible by the administration of two or three full doses of vaccine at seven-day intervals, say, every four months.

I have in this way kept entirely free from all catarrhal attacks during the past three years a well-known personage who first consulted me at the age of eighty-four. His pneumococci have never, so far as I know, entirely gone, and I have considered it my duty to warn him that sooner or later they will make their presence felt if he allorrs his immunity to fall unduly.

Another important point in such cases is that the factor which, above all, probably decides the lighting into virulence of the dormant pneumococci is the incidence of a fresh infection by some other catarrhal micro-organism; chief among these is undoubtedly the B.infuenza, probably also the streptococcus and M. catarrhalis, and it is owing to this fact that an epidemic of influenza is nearly always attended by a greatly raised mortality, due to acute pneumonia. At the same time it must be admitted that a pure invasion of any part of the respiratory tract by the B. infuenze is comparatively rare; there is nearly always some other associated microbe and this is usually the pneumococcus, so that a certain percentage of the cases of pneumonia encountered during a socalled influenza epidemic are really due to a fresh infection by the pneumococcus associated with the $B$. influenze. In such cases the employment of the suitable raccine in the first stages of the infection before the bacteria have actually located themselves in the pulmonary tissues would, I renture to affirm, almost entirely prevent the onset of pneumonic attacks, and many valuable lives would thus be saved at every such epidemic.

\section{The Pathology of Acute Pneumonia and its Bearing on Vaccine Treatment.}

Before it is possible to use vaccines in this condition with any prospect of achiering thoroughly satisfactory results, it is essential to 
have a perfectly clear idea of the pathology of the disease and how recovery is brought about.

In the first stage, that of congestion, the capillaries are dilated and tortuous from distension with blood; the air-cells are nct as yet completely airless, but are more or less filled with frothy reddish serum albumen; the supply of opsonin and other anti-bodies in the circulating fluids is below the normal, the leucocytes as a rule considerably above the normal.

During the second stage, known as red hepatisation from the

Chart Xili.

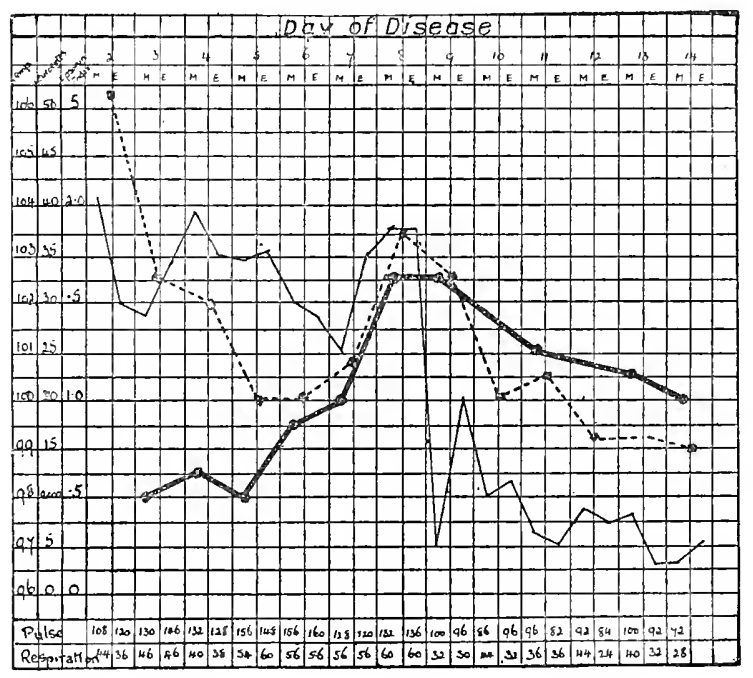

Relation between Leucocytes, Opsonic Index, and Temperature in a Case of Pneumonia (Eyre).

Dotted line $=$ number of leucocytes per cubic millimetre; thick line $=$ opsonic index; thin line $=$ temperature.

resemblance which the lung then bears to the liver, the organ is of a dull red colour, finely granular in section, completely airless, solid, and sinks in water. The contents of the alveoli consist of coagulated fibrin which holds in its meshes red blood-cells, leucocytes, exfoliated epithelium and bacteria. Although the capillaries are much compressed, they for the most part remain pervious. The supply of antibodies remains low, and the leucocytes in the circulating blood have fallen considerably, although they may still be above the normal; this fall may be due to the vast numbers stored up in the pulmonary tissues.

The stage of red hepatisation passes into that of grey hepatisation, the change of colour being brought about by the extravasated red corpuscles losing their hæmoglobin, by the stasis of the circulation 
through the capillary vessels of the alveoli, and by the increased number of leucocytes which crowd the air-cells and alveolar walls; the leucocytes in the blood-stream have now fallen to their minimum.

In cases which pursue a normal course, this stage of "grey hepatisation" so rapidly passes into that of "resolution" that it may be regarded as the stage of commencing resolution; the leucocytes and exfoliated epithelium undergo granular and fatty degeneration, the fibrin softens and is absorbed, the capillary circulation becomes actively re-established, the alveoli again contain air, and the alveolar epithelium

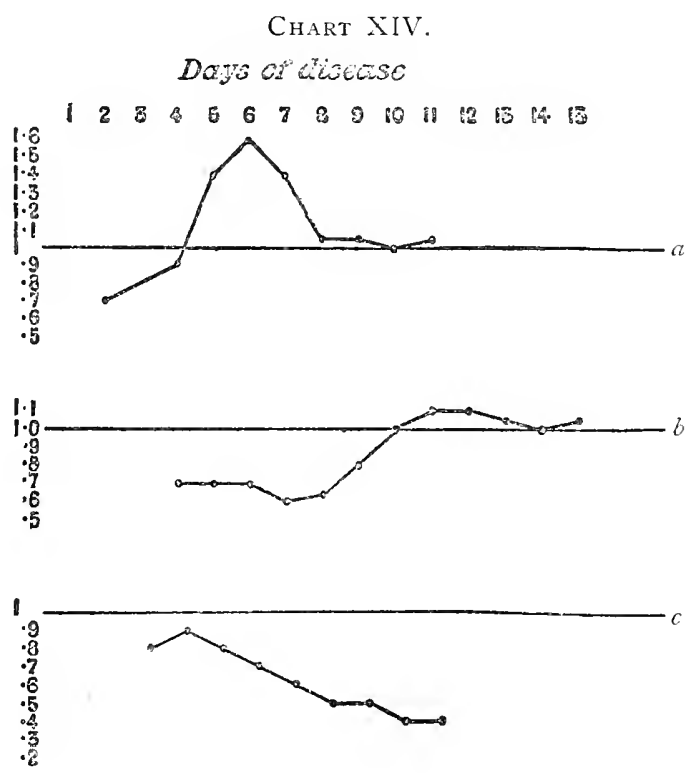

Types of Reaction of the Opsonic Index in Pneumococcic Infection (AFTER EYRE).

(a) Immediate rise as seen in mild infections; (b) delayed rise; (c) progressive decline as seen in severe and fatal infections.

is regenerated. The commencement of this process of resolution would appear to coincide in point of time with the sudden fall of temperature, occurring in nearly 50 per cent. of cases, known as the "crisis," and with the sudden rise of leucocytes and immune bodies in the general blood-stream. The annexed chart, No. I3, of Dr. Eyre"s shows well the relationship between temperature, leucocytes and opsonic index at the various stages of the attack; while chart No. I4 shows the course of the opsonic index in cases $(a)$ in which the fall of temperature is by crisis, $(b)$ where it is by lysis, $(c)$ where the case proceeds to a fatal issue.

The chief factors concerned in the processes of cure are (I) leucocytes, (2) endothelial cells of the alveoli, (3) opsonins, (4) antitoxins, 
agglutinins and other immune bodies. As we have seen above, the opsonic index yields a very fair indication of the progress of immunity, and the extinction of the infective agents would appear to depend largely upon the action of opsonin which is chiefly of the thermolabile variety. The phagocytosis, however, is not, as might be supposed, carried out in the main by the polymorphonuclear leucocytes, which, as we have seen, have so largely increased in numbers at or just before the crisis, but, as De Jong has pointed out, by the endothelial cells of the alveoli, many of which become transformed into macrophages. The chief rôle of the leucocytes would appear to be the formation and excretion of ferment for the solution of the fibrin filling the alveolar cells; this would appear to be the explanation of the great storing up of leucocytes in this locality.

It is now well recognised that the rapid respiration and high temperature so characteristic of pneumonia are due to a poisoning of the nerve-centres by the endotoxins liberated by the death of the pneumococci, this same agent being also responsible for changes in the musculature and nerve control of the heart, which may lead to circulatory failure even when convalescence appears to have been well begun. The satisfactory elaboration of the corresponding antibodies is therefore a very essential factor for recovery, and the production of the "crisis" is dependent upon the elaboration of such antibodies, which appear to be inoperative until they reach a certain concentration; in such cases it seems that the reaction between toxin and anti-toxin must be "all or nothing," and so bears no resemblance to the neutralisation "in vitro" of diphtheria toxin by antitoxin. In cases where recovery is by lysis the neutralisation must be more gradual.

Let us now consider what bearing these various facts have upon the application of vaccine treatment to this disease. Our objective must be a two-fold one-to kill the bacteria and to neutralise their toxin. The question is, Are these possible of attainment? The answer is, This depends largely on the stage of the disease, the virulence of the infection and the responsive powers of the individual.

In the stage of pulmonary congestion the bacteria are circulating in the blood-stream, and are present in but scanty numbers in the pulmonary tissues; the resisting power of the body has been obviously broken down, and bacteria circulating in the blood-stream do not appear to be capable of leading to the elaboration of antibodies; there is thus both a local and general defect of these, but at the same time the amount of toxin formed is not considerable. If, therefore, the bacteria could be exterminated the disease process would be ended. It is now well recognised that the introduction into healthy subcutaneous or muscle tissues of a therapeutic dose of vaccine will lead to the 
speedy elaboration of the corresponding antibodies. A vaccine of pneumococci is but slightly toxic, and its administration leads to the elaboration of considerable amounts of opsonin, which is chiefly of the thermostable variety, and of a small amount of antitoxin. The fact that this is precisely what is then required is the strongest possible argument for the employment of a vaccine at the very inception of the attack. It is true that the virulence of the infection then is high, but so is the responsive power of the individual. Unfortunately there are two obstacles to this procedure: (I) the fact that the opportunity does not always present itself, many cases not being seen till the disease processes are further advanced; (2) the refusal of medical men to recognise every case of pneumonia as potentially a fatal one.

When consolidation has occurred bacteria are still often to be found in the blood-stream, but their multiplication in the pulmonary tissues has much advanced: here they are enclosed in a meshwork of fibrin, in a warm nidus admirably suited to their growth; the blood and lymph supply being more or less in a state of stasis, even if the amount of immune bodies in the body generally is more than adequate these are not available, or only to a limited extent. No immediate material good might therefore be anticipated from increasing them by means of a therapeutical inoculation; that good does sometimes result therefrom is due to the fact that all portions of the infected lung are not in the same stage; one area may be in a state of congestion, another in that of red hepatisation, and yet a third in that of commencing resolution. The main objective, therefore, is to relieve the strain on the heart's musculature as much as possible by lowering the viscosity of the blood with full doses of citric acid, by dilating the blood-vessels locally by the application of heat, etc., and by relieving the general blood-pressure by promoting free diaphoresis and action of the bowels, and if necessary by bleeding. At the same time we may increase the amount of antibodies, both antitoxic and antibacterial, and the number of leucocytes against the time when commencing resolution may enable them to be carried to the battle front. Resolution will inevitably entail the free liberation into the re-established circulation of quantities of toxin which have been stored up in the consolidated tissues; hence it is very important now to ensure that the leucocytes and various antibodies can reach the infected areas with the least possible difficulty, and to preserve the heart musculature as much as possible from the action of the bacterial toxins. Were a really reliable antitoxic serum available for the neutralisation of the toxins, the combination of serum and vaccine could hardly fail to prove of the utmost value during this period of resolution. Unfortunately the best available sera, Romer's and Pané's, appear to leave much to be desired; their defects may, however, be 
more apparent than real, failures with them being perhaps as much due to their improper use-viz. insufficient dosage and non-systemic administration-as to any intrinsic imperfections.

The administration of a vaccine when resolution is impending will prove of inmediate service provided that-

(I) The supply of anti-bacterial bodies is otherwise insufficient.

(2) The tissues are capable of responding to stimulation, and this they almost invariably are.

(3) The heart mechanism is sufficiently strong to withstand the attack of the toxins so copiously liberated during this stage.

As we have seen, the production of the "crisis" is due to the massive neutralisation of toxin by antitoxin. Hence it is that a small dose of vaccine may at one time suffice to produce an immediate reaction as evidenced by crisis occurring and at another time may appear to fail. The power of a vaccine to stimulate the formation of antitoxin is but slight; hence if the defect in antitoxin is small the addition of a small quantity will suffice, but if the defect be great a much increased dose of vaccine may fail to produce the additional amount requisite to make good the considerable defect; the total amount of antibody then present being the sum of the amounts produced so far by natural means plus the amount produced by the inoculation of the vaccine requires further supplementing, either by the natural response of the body to the infection or to the stimulation of another dose of vaccine, or by both these means.

As has been already mentioned, when the fall of temperature is by lysis this massive neutralisation of toxin would not appear to occur, and it is worthy of note that the proportion of cases in which "lysis" occurs rather than "crisis" appears to be greater in vaccinated than in unvaccinated cases.

These conclusions may be briefly summarised as follows:

(I) The administration of a vaccine in the earliest stages of a pneumonic attack can hardly fail to be productive of good in a very considerable percentage of cases, for thereby is ensured the formation of adequate bactericidal bodies, and these are precisely those which are of service at this period of the disease.

(2) The administration of a vaccine during the period of consolidation theoretically can hardly be productive of immediate good, but the resultant immune bodies may prove of considerable service once "resolution" has begun. Owing, however, to the fact that the stage of the disease may vary considerably at different foci, the good resulting from vaccine treatment may be much greater than might be anticipated. I cannot help feeling that these are the cases where the fall of temperature is by "lysis." 
(3) The administration of a vaccine during the period of resolution will prove of service especially if resolution be delayed, and if the supply of bactericidal bodies be otherwise insufficient.

It must, however, be clearly borne in mind that no vaccine treatment will prove of service unless-

(I) The vaccine be the proper one and be properly prepared.

It is no use employing a pneumococcal vaccine in a streptococcal or Friedlander infection, nor is it any use employing a vaccine devoid of immunising properties. I have already mentioned that some strains of pneumococci yield a good vaccine only when heat is used to ensure sterility, others only when sterilised with antiseptic.

Experience has also shown that an autogenous vaccine is preferable to a stock one, but there is much to be said in favour of beginning treatment immediately with a stock polyvalent vaccine of proved immunising powers. While some avirulent strains of the $B$. diphtheriae yield the most potent antitoxin, and some strains of the $B$.typhosus of very low virulence the most powerful vaccine, with the pneumococcus the more highly virulent the strain as a rule the more potent is the vaccine. The pneumococci isolated from a given case of pneumonia may vary much in virulence amongst themselves; it is, therefore, obvious that in the preparation of an autogenous vaccine, cultures should be taken, not from one, but from numerous colonies.

(2) That adequate dosage be employed.

Two things that have especially struck me in reading the clinical histories of cases reported by those who have failed to secure satisfactory results have been-(a) the inadequate dosages: for instance, in the record of nineteen cases by one observer I find that nine cases did not receive a dose of even 5 millions, and only one case a dose of 25 millions; this is little more than playing with vaccine treatment.

(b) The tendency to reduce subsequent dosages when the patient fails to make any response to a first dose, which, in fact, was itself quite an inadequate one judged by the experience of all those who have achieved good results. Failure to respond in any way to a given dosage is an indication, not for reduced, but for increased subsequent doses.

I might also add that increased toxæmia has been at times regarded either as a bad effect or as indication for diminished dosage-in reality it may be neither. It is an indication for promoting neutralisation and excretion of the toxins by every available means, and it may also be indicative of hastening resolution and consequent increased absorption of the toxins into the general circulation, but it certainly is not indicative of postponing further inoculation or of diminishing the dosage: is may be so in the case of some infections, but above all, is this not the 
case with pneumococcal infections? The toxic contents of a pneumococcal vaccine are so small that the amount so introduced into hea thy tissues is without effect on the circulating mass, and only leads to the elaboration of the corresponding antitoxin, while the stimulus to the formation of opsonin, and so to the phagocytosis of the bacilli and neutralisation within the macrophages and polynuclear cells of their endotoxins, is very great. Personally, I have never seen a pneumococcal vaccine do any injury to a patient; per contra I have seen several cases of persistent hyperpyrexia with low muttering delirium, one or two apparently comatose, make almost immediate response to an inoculation of 25 or 50 millions-it is true that these were cases with marked bacteriæmia, but this I think they often are.

In conclusion, I will briefly summarise the procedure which has been found by most experienced observers to be the best, and give a short resume of the results they have obtained.

\section{Procedure.}

(I) Take sputum smears and cultures: if in early stage bloodcultures, if in advanced stage perform a lung puncture, and proceed to preparation of an autogenous vaccine.

(2) Immediately administer a dose of not less than 25 millions of a polyvalent stock vaccine, prepared from virulent strains and of proved immunising power.

(3) If no definite response within thirty-six to forty-eight hours, repeat above dose of stock vaccine, or preferably of the autogenous vaccine if this be ready.

(4) If still no response, administer double the above dose in thirtysix to forty-eight hours; if response, as evidenced by improved clinical signs and symptoms and increased well-being of the patient, deferre-inoculation for three days, or until the first signs of retrogression in the general condition or clinical signs and symptoms.

(5) Maintain dosage or even increase it, and continue inoculations at intervals of three to four days, until the patient is perfectly well.

(6) If the patient at any time takes a sudden turn for the worse, make diligent search above all for such complications as may require surgical interference. It may be advisable in such conditions to suspend specific treatment, but immediate reinoculation, perhaps with an increased dosage, may be more likely to be productive of good.

(7) Use every known means throughout of securing toxic elimination, of sparing the heart from needless strain, and of tiding it over the period of greatest stress.

Resume of results obtained by various observers and their conclusions.- 
Although I have always been one who thinks that far too much regard can be made in medicine to statistics which may be inherently fallacious, yet none the less it is certain that every form of treatment will be judged by the case mortality and incidence of complications experienced during its employment. The pathogenity of the Pneumococcus and so the death-rate varies greatly in different epidemics, and such factors as age and previous habits of the individual are well known to be important prognostic points, so that if statistics are to have any value an adequate number of truly comparabie cases treated upon orthodox lines are essential to serve as controls-this unfortunately is not always possible, and where it cannot be done the assumption must be made that the death-rate would have otherwise approximated to the normal, which may be taken as between $\mathrm{I} 6$ and 20 per cent.

Wolf (Journal of Infectious Diseases, I9o6, p. 739) treated I4 cases with vaccines during an epidemic in which the total mortality was 40 per cent.

II of the I4 cases, or 78.5 per cent., recovered.

3 , , , $2 \mathrm{I}$ '5 ", died.

In Io of the II cases which recovered the crisis occurred within thirty-six hours of the first inoculation.

Leary (Boston Med. and Surg. Joum., I9o9, p. 7 $\mathrm{I}_{4}$ ) records his results in 83 cases. Of these, 34 were alcoholics in whom the normal death-rate was 50 per cent. Of these 34 cases only 6 died-a mortality of I 7.7 per cent. Of the other 49 cases only 2 died - a mortality of only 4 per cent. The death-rate among the whole $83=9.7$ per cent.

Rapid relief of toxæmic conditions was noted, and in cases of otherwise uncontrollable delirium the abatement thereof was prompt.

Craig (Medical Record, November I8th, I9II) records his results in 20 cases among old sailors, all over 60, most much older $(80-90$ years old). Most were alcoholics, nearly all had chronic nephritis, arteriosclerosis and dilated hearts. The average death-rate in the institution for the preceding five years from pneumonia had been 66 per cent. Yet of these 20 only 4 died, a death-rate of 20 per cent., and of these 4 only I died directly from the pneumonia, this being a very severe case of bilateral disease; of the other 3 cases one was already complicated by purulent pericarditis, and another by acute uræmia and acute dilatation of the heart.

Charteris (Glasgow Med. Journ., January, I9I2, p. I9) obtained much less favourable results in I9 cases. Ten simultaneous cases which seemed on the point of crisis were used as controls. His conclusions were that the administration of a stock pneumococcal vaccine had no marked effect upon the subsequent course of the disease, the mortality 
being practically identical-2o per cent. in the two series-that the early administration of vaccine did not abort the disease nor prevent complications, and that complications were relatively frequent in the vaccine series, viz. I case of meningitis, 2 of empyema, and I of hyperpyrexia.

It is very easy to be critical and perhaps do injustice to other people, but a study of the paper leads to the following criticisms: (I) That the use of cases obviously about to crisis as controls is quite inadmissible.

(2) That of the four cases which died two were alcoholics who were delirious or semi-conscious when treated.

(3) That in only eight of the nineteen cases was a dosage of Io millions of a stock vaccine exceeded; in eight of the cases it was under 5 millions.

(4) That in two of those that died an initial dosage of 20 and I8 millions respectively was reduced subsequently to 10 and 2 millions respectively, although all the indications that can be gathered from the clinical histories were for maintaining or even increasing the dosage.

For these reasons I regard his conclusions as fallacious, his controls are inadmissible, and all that the paper shows is that the administration of a stock vaccine in doses already well known to be inadequate failed to reduce the mortality below 20 per cent.

Parry Morgan (Proc. Roy. Soc. of Med., vol. iii, No. 9, Supplement, p. I65) treated 43 cases with 2 deaths-a mortality of 5 per cent.; one of these two died from nephritis after the pneumonia had subsided. Treatment was begun at periods of the disease from the second day onward, some cases being treated with stock vaccine, some with autogenous; one of these latter cases proved to be a streptococcal infection. In many cases he repeated doses of 50 millions of autogenous vaccine and never saw any harm result, but he inclines to the view that a dosage of $I 5$ to 30 millions gives the best result in the average adult. When an artificial crisis was not produced the temperature often fell by lysis, with marked improvement in the symptoms. For seven of the most interesting cases, as being complicated ones and the most convincing the original account should be consulted.

From the experience gained in the treatment of these cases, Morgan says that the temperature may be a guide but often is not, one of the most noticeable features being the improvement in the general condition without much change in the temperature; the anxiety which one often feels for a patient is relieved, sleep comes readily, the appetite improves, and even if the pulse-rate does not fall its strength is well maintained. At other times there is a fall in the temperature soon after the dose, sometimes even in a couple of hours; if this is the case another dose is indicated when the temperature rises again. 
If there is no change in twenty-four hours the dose should be repeated, sometimes being increased, but more often decreased; between subsequent doses a longer interval may be left. For various reasons he finds the opsonic index is frequently quite unreliable as a guide to the progress of immunity in pneumonia, and feels that some other measure of it is much to be desired.

Butler Harris (Brit. Med. Journ., June, Igog, p. I530, and Proc. Roy. Soc. of Med., vol. iii, No 9, Supplement, p. Io3) considers that-

(I) Successful inoculation for pneumonia is possible.

(2) Inoculation does no harm.

(3) A vaccine from one or a number of virulent strains should be used.

(4) It should be introduced as early as possible.

(5) The estimation of the opsonic index is not necessary.

(6) The observation of the temperature and physical signs is a sufficient guide to the repetition of the dose.

(7) Infections of the lung by the pneumococcus which fail to resolve after an acute pneumonia, as well as pneumococcal infections of other areas, ought certainly to be treated with a vaccine.

He found that a dose of 20 to 50 millions might be given without harm, that usually a fall of temperature was produced in a few hours. that frequently it rose again, but not to the same level, and that it was often necessary to repeat the inoculation once or twice. He is convinced that usually a distinct reaction in favour of the patient is produced, and that it is wise to begin inoculations before the nerrous mechanism suffers much inhibition. In the sequelæ of pneumonia he considers the use of a pneumococcal vaccine as sure of a good result as that of a staphylococcal one in case of boils.

After comparison of the clinical histories of his cases with those of Craig and of Parry Morgan he finds that the effects seen by each of them as produced by bacterial inoculations in cases of pneumonia are practically identical, and considers that every case of pneumonia should be from the outset regarded as possibly a fatal one, and suggests that a stock vaccine of proved immunising power should always be employed as early as possible in a dose not exceeding 20 millions, and that the preparation of the autogenous vaccine should be at once proceeded with.

This collective evidence is surely sufficient to warrant the unprejudiced in giving a fair trial to vaccine treatment of cases of pneumonia. What he is entitled to expect and what he is not entitled to expect therefrom has been fairly laid before him. It only remains for him to pay due heed to the various considerations, to utilise common-sense and clinical experience. and to be prepared to judge results with due impartiality. 


\section{Unresolved Pneumonia.}

As we have seen, the leucocytes are held to be the chief agent in the production of resolution by means of ferment which they liberate; hence the rational procedure in a case of unresolved pneumonia would seem to consist in the promotion of a leucocytosis, and the determination of a leucocyte flow to the infected area by fomentations, blisters and other means. Whether it has been actually observed that cases of acute pneumonia showing a relatively defective leucocytosis are especially apt to resolve badly, and that there is a leucopænia, relative or absolute, present after failure to resolve, I know not; observations upon these points might perchance repay the clinician for his trouble. Furthermore, as we have already seen, it is hardly reasonable to expect that the administration of a vaccine will have much immediate effect during the stage of consolidation in an acute attack. For these two reasons it would seem hardly reasonable to expect that a vaccine should have much immediate effect upon an unresolved condition. The blood and lymph stasis is, however, not then so complete as at the height of consolidation, some attempt has been made at the re-establishment of the circulation. It therefore follows that some good may be expected to accrue from a course of vaccine treatment. It must, however, be remembered-

(I) That even if the infection were an unmixed one at the inception of the attack it is hardly likely to have remained so. The streptococcus or $M$. catarrhalis will almost certainly complicate the picture, and to these attention may have to be directed. In the few cases which I have myself investigated a mixed infection has been always present, and I have thought it the better practice to employ a mixed vaccine.

(2) That progress necessarily must be somewhat slow.

(3) That the production of local reactions in the infected tissues is essential to cure, and that such dosages must be used as will produce this effect. A 50-million dose of any of the above three organisms will do to begin with, but should stethoscopic observations and clinical symptoms show failure of local reaction this dosage should be increased. Personally I have found ultimate doses of 500 millions essential to complete cure.

Periodic examinations of the sputum should be made to check progress and eliminate the possibility of a new infection having been incurred, and to ensure the continuance of treatment until the pneumococcus has entirely disappeared from the sputum.

In one very interesting case of mine it took four months' treatment to bring about a satisfactory clinical condition, and an additional eight months to secure the desired bacteriological result. 


\section{Empjema, Lung tbscess, etc.}

There is nothing specially to mention as regards the vaccine treatment of these conditions. Treatment will be guided by the considerations which govern the procedure in all cases of abscess formation and of sinuses in the body generally.

Prevention, as always, is better than cure, and that a considerable reduction is effected in the incidence of these and other complications by the early application of vaccine treatment to all cases of pneumonia is agreed on by all who have had mature experience of this procedure.

In the case of lung abscesses especially is the bacteriology apt to become a very complicated one as times goes on. Streptococcus. M. catarrialis, B. coli, B. proteus, B. pyocyaneus, B. influenze, $M$. tetragenus, and $B$. of Friedlander are a few of the accessory microbes which may make their appearance, and in the preparation and use of the autogenous vaccines considerable discrimination and care may be required. Free drainage is a sine qua non to cure in cases with external openings, and there might seem to be reasonable grounds for the fear that vaccine treatment might bring about closure of the vent or sinus, and induce local accumulations of pus. In practice it would appear that this danger may be easily exaggerated. I remember one patient whom I was treating with a mixed pneumococcus, streptococcus, $B$. infiuenze vaccine for chronic antral trouble, who was also suffering from an old lung abscess, which sometimes dis. charged by bronchus as well as through a persistent external sinus, expressing great anxiety when he found that what he regarded as his safety vent was closing up. I reassured him and treatment was continued, with the result that he has not lost a day's work for two years, and that antrum and lung abscess have caused no further trouble. None the less is it wise whenever possible to make sure that any sinus is healing from the bottom by occasional probing and the instillation of citrated salt solution.

Small dosages are best at the beginning of treatment, but as time goes on they should be increased with boldness; a slight constitutional reaction and alteration in the character and amount of discharge are the best indications of adequate dosage.

\section{Broncho-pneumonia in Children.}

This condition will, I venture to predict, prove one of the most profitable of all fields for the worker in vaccine therapeutics. Ignorance of what constitutes the defensive mechanism in young children against bacterial invasion has led to a somewhat natural reluctance to apply 
methods applicable to the adult. The results achieved in the treatment of whooping-cough, gonorrhoal vulvo-vaginitis and conjunctivitis show clearly, however, that children, and even infants, do respond readily to therapeutic inoculation.

In broncho-pneumonia we have a disease of such high mortality that ample justification exists for endeavours to find some specific form of treatment. The consolidated areas are not, as a rule, so large that any considerable stasis of the circulation results, as in the case of lobar pneumonia, and upon this fact additional hope of success may be based.

Peculiar difficulties, however, present themselves. The bacteriology is a much more variable one than in the case of lobar pneumonia. Pneumococcus, streptococcus, B. influenza, B. of Friedlander and perhaps the staphylococcus and other organisms may be responsible for the condition, either singly or in any combination. If vaccine treatment is to be resorted to, it is obvious that the precise nature of the infection should be determined. Unfortunately children are very apt to swallow sputum, and it may be impossible to get specimens for examination; if they can be got, then all, so far, is well; if they cannot, lung puncture is also liable to prove a failure owing to the small size of the consolidated areas; blood-cultures might prove of service, but so far as I know no observations have been made in this direction. If specimens of sputum can be obtained, the best procedure would appear to be to make examination at once of stained smears, and administer stock vaccine of proved immunising power corresponding to the infection found while the autogenous vaccine is in course of preparation. If no specimens are obtainable and lung puncture and bloodculture fail, then I think it will be more than justifiable to employ a stock vaccine of the following composition: In each c.c., pneumococcus, Io millions; streptococcus, Io millions; $B$. influenze, 20 millions; B. of Friedlander, 20 millions; the dosage to be, under three years, 2 minims; three to five years, 4 minims ; five to seven years, 6 minims; over seven years, 8 minims, the indications for repeating or increasing the initial dosage being general condition in the first place and temperature in the second. As a general rule young children bear relatively high dosages of vaccine extremely well.

Of published results achieved in broncho-pneumonia there are none as yet available, but one or two private communications which I have received are very favourable to this therapy. 


\section{CHAPTER IX.}

\section{VACCINES IN WHOOPING-COUGH, DIPHTHERIA, PYOR- RHCEA ALVEOLARIS, HAY-FEVER, OZENA, AND RHINOSCLEROMA.}

(a) Whooping-cough.

That the bacillus of Bordet-Gengou, described on pp. 3 I and 32 , is the true specific cause of whooping-cough is now generally accepted, the various serum reactions affording strong confirmatory evidence. Early in the attack the Bordet bacillus appears in great numbers in the secretions, but the careful investigations of Freeman and of Martha Woolstein into the bacteriology of this disease have shown that even then it has very frequently associated with it the $B$. infuenza, and often the pneumococcus; the great liability to such complications as bronchitis and broncho-pneumonia is thus explained in precisely the same way as the liability of so-called influenza attacks in the adult to develop into acute pneumonia.

As early even as the end of the second week the Bordet bacillus has almost disappeared from the secretions, whereas the B. influensee and pneumococcus, and perhaps the streptococcus and M. catarrhalis. have increased considerably in numbers. A pure infection is so rare an occurrence that the symptoms referable to the Bordet bacillus alone may be almost said to be unknown. The bearing of this upon the vaccine treatment of whooping-cough will be dealt with presently, The first attempt at the establishment of immunity to the disease was made by Bordet about ten years ago. He inoculated twelve healthy children with large doses of a vaccine; very soon afterwards they came in accidental contact with a case of whooping-cough, with the result that they all immediately fell victims to very severe attacks of the disease. The explanation of this occurrence is now taken to be that contact took place during the period of lowered resistance (or "negative phase") Gonsequent upon the use of very large doses of the vaccine. This view presented itself to Freeman, and on p. 97 of the Proceedings of the Royal Society of Medicine, vol. iii, No. 9, supple- 
ment, he gives a detailed account of the many careful observations which he made in order to determine the correct therapeutic dose. Doses varying from 2 to 220 millions were employed, the final conclusion being that a dosage of about too millions gives the best results, and that a weekly repetition is quite safe. By these means he found that the average duration of the disease in his vaccinated cases was reduced to 4.3 weeks as compared with $7^{\circ}+$ weeks in those who received inoculations of saline solution and served as controls. It must be remembered that many of these cases were treated with dosages which Freeman now recognises to be inadequate, and that no specific treatment was directed against the complicating organisms, such as the pneumococcus and B. inflienza. This he now advises shall be done as a matter of routine, and my own limited personal experience is entirely confirmatory of the correctness of this procedure. The following scheme of dosage may be safely followed :

Age.

Under I year

I-2 years.

$2-3 \quad$, .

3-7 ,

Over 7 ,"
B. Bordet. B. influenza. Pneumococcus.

$\begin{array}{rrrrr}25 & \cdot & \text { IO } & \cdot & 2 \\ 50 & \cdot & 25 & \cdot & 5 \\ \text { IOO } & \cdot & 50 & \cdot & 5 \\ \text { IOO } & \cdot & 50 & \cdot & \text { IO } \\ \text { IOO } & \cdot & \text { IOO } & \cdot & \text { IO }\end{array}$

Reinoculation may be performed at intervals of five to seven days, and at the third inoculation a double dosage may be employed if thought advisable.

As a prophylactic the initial dosage corresponding to the age may be doubled in seven days; and this again doubled if thought advisable after another seven days.

The employment of the above combined vaccine for this purpose may also be expected to diminish the liability to broncho-pneumonia, and is devoid of all risk.

As to the duration of the immunity thereby conferred we as yet know nothing; probably it is about six months, so that two series of inoculations yearly may suffice to confer the desired protection.

Saunders and collaborators have described (Pediatrics, March, Igr2) their results in forty cases of whooping-cough and in fourteen children who had been exposed to contagion. They also began with small doses, viz. 5 millions, but soon concluded that the dose was too small and increased it to IO-20 millions, repeated as required. Despite the fact that this increased dosage is still an inadequate one, they obtained results which led them to the following conclusions:

(I) That as a prophylactic the vaccine has a decided value; that whilst it is true that vaccination or some other infection will postpone 
or interrupt the course of pertussis, vaccine alone will absolutely prevent it. The immunity is of uncertain duration, but the injections may be repeated and it is of the utmost importance to postpone the disease until the child has passed the age of two. The failures reported by some observers must be attributed to an impotent vaccine or to insufficient dosage.

(2) That as a remedial agent success depends upon the promptness of administration and the freedom from complications at the time.

(3) In no case should other treatment be withheld if indicated, especially in infants, who may be spared convulsions or broncho-pneumonia by the use of emetics, sedatives, or some aromatic compound.

(4) It is quite possible that much better results may be obtained in late cases by the use of larger doses (and the combination of vaccine directed against the allied organisms).

(5) That in view of the high mortality from pertussis in young children there should be a systematic effort made to determine the duration of artificial immunity and to keep them protected.

\section{(b) Diphtieria.}

Diphtheria from the point of view of the raccine therapist is a disease exhibiting several points of especial interest, and it is therefore singularly strange that so little attention has been deroted to its study in this direction.

In the first place we are in some doubt as to wherein lies the defensive mechanism of the body against the $B$. diphtheria. Wright has stated that there is no opsonin in the body fluids for this bacterium. Ruth Tunnicliffe, however, finds that the process of recorery runs parallel to, and is due to a rise in, the opsonic index, and that the main factor in the cure of the disease is the remoral of the bacilli by phagocytosis and consequent cessation of the absorption of toxin. Antitoxin formation seems to play a very subsidiary part, for recovery is often well advanced before antitoxin can be detected in the blood-in fact it is sometimes apparently absent throughout. Emery (Immunity and Specific Therapy, p. +09) says: "The observations referred to previously show clearly that the process of cure of the local lesions is assisted by the production of an opsonin. And there is every reason to believe that it is by phagocytosis that the bacilli are combated, bacteriolysis being very doubtful and of comparatively small importance. The cure of the disease is dependent, therefore, partly on antitoxin formation and partly on phagocytosis." Against this, however, is the fact that phagocytosis of the diphtheria bacillus is very rarely seen in smear preparations made from the local lesion. 
In the second place this is a disease in which conspicuous success has attended the use of antitoxin serum both in the direction of diminished mortality and of diminished complications. This serum, however, appears to be solely antitoxic and quite devoid of anti-bacterial properties.

In the third place recent investigations are showing more and more clearly how important are so-called "carriers," i.e. those in whom the bacilli persist locally after the subșidence of all clinical symptoms in the initiation of diphtheria epidemics.

The statement has been made that other bacteria, such as the streptococcus and staphylococcus play an important part in causing the disappearance of the $B$. diphtheria from the throat, etc., after an acute attack, and the proposal has emanated, I believe from Germany, that the process may be hastened by spraying the infected parts with living broth cultures of the Staphylococcus aureus. Good results have been reported by one or two observers, but I cannot but feel that the method is fraught with danger and is quite unwarrantable.

Walton Smith (Australian Med. Gaz., October 2oth, IgIo, p. 543) gives details of the vaccine treatment in the case of a girl in whose throat the $B$. diphtherice persisted for fifteen weeks after antitoxin was given, recovery being otherwise complete. The first inoculation with 6 millions $B$. diphtherice and Io million staphylococcus (as this organism was also present) resulted within twenty-four hours in the production of a well-marked reaction at the site of injection, in some general disturbance and a temperature of $100^{\circ} 2^{\circ} \mathrm{F}$. A week later the bacilli were still present, so an inoculation of 8 million $B$. diphtherice was given. The reaction was very slight on this occasion. Subsequent examinations of the throat secretion failed to show the presence of any Klebs-Loeffler bacilli.

The suggestion that vaccine treatment might be combined with antitoxin seems reasonable, especially in cases where the bacilli tend to persist, and there is some ground for hoping that late complications may thereby be minimised. It must be left to future observation to decide whether further combination of a vaccine of the predominant allied organism, streptococcus, staphylococcus or M. catarrhalis, is also advisable. The initial dose of the diphtheria vaccine should not exceed 5 to Io millions for the present; future observations may, however, show that higher dosages are advisable and devoid of danger.

(c) Pyorrhea Alveolaris.

A very great amount of bacteriological research has been devoted to this disease, and numerous reports have now been published of the results of vaccine treatment. These are almost without exception 
highly favourable, but I must confess to considerable scepticism. In the very earliest stages, before pus pockets are definitely formed, strict attention to oral hygiene, careful local treatment by a thoroughly competent dentist who has time to devote to tedious scaling and polishing, perhaps combined with vaccine treatment, may suffice for cure; by "cure" I mean cure beyond recurrence, provided that the patient does his duty for the rest of his natural life and pays a visit every six months to a competent dentist. But when the condition is definitely established, when careful pressure around the gum margins results in the appearance of pus-sure sign that there are pockets, perhaps an eighth of an inch deep or more-it is another matter. That bacteria are resident in the tissues of the gum is true, and that vaccine treatment may suffice to eradicate these is also true, but not all the vaccine treatment on earth can ever influence those lying in the space between the tooth and separated tissue of the gum. Scaling of tartar, polishing the teeth so far as this is possible, careful brushing night and morning of the teeth with a moderately hard brush and an acid antiseptic wash, such as "albodent," and of the gurns with a softer brush, followed by massage with the finger-tip and eau-de-cologne, will all help, but these likewise will not suffice to re-establish a normal condition of the parts; and when I read that vaccine treatment combined with much less local care than this suffices to tighten loose teeth and bring about elimination of the pus pockets, I am a grave doubter of the writers' powers of clinical observation. But, it will be said, is there no cure of this most important disease, a disease fraught with such serious immediate and remote dangers to the patient's health? To this my answer is, "Certainly, in a considerable percentage of cases," but it requires a skill and expenditure of time which few dentists are prepared to devote to it. The fault is not altogether theirs; the public are as much or more to blame, and the medical profession cannot be considered to be exempt from responsibility. The public are ignorant of the consequences of the disease, and, being ignorant, are ill-disposed to pay adequately for its cure. They go to the dentist when their teeth hurt them, and are prepared to have cavities filled. The dentist sees or fails to see a serious condition of pyorrhœa; if he sees it he often fails to tell the patient of its presence and to urge its treatment, for he fears to lose his patient. The medical man hardly ever knows pyorrhœa when he sees it ; it is a disease rarely described in the text-books, and his teachers in student days failed entirely to demonstrate it and to impress its far-reaching consequences upon him; if they did they would only stultify themselves in the students' eyes, for after all their talk they would perforce have to leave the condition practically in statu quo, for no facilities exist at general hospitals for its adequate treatment. The position is a very serious 
one, but it is not easy to find the remedy; perhaps the movement for the systematic examination and treatment of school-children's teeth is the best that is available; the misfortune is that the condition is often established even at this early age.

As such measures as I have already mentioned will most likely fail to secure complete cure resort obviously must be made to other means. Among these are the following:

(I) Strictly local treatment of the tissues in the pockets.

(2) Attention to such other oral conditions as follicular tonsillitis and post-nasal catarrh.

(3) Perhaps combined with these the use of appropriate vaccines.

Let us consider these in turn: by (I) I mean the systematic removal of the contents of the pockets and the topical application of such medicaments as will lead to the removal of tissues diseased beyond repair, to the stimulation to repair of such tissues as are capable of repair, to the obliteration of the pockets and the complete reapproximation of tooth and gum. There are limitations to success by these means, the chief causes of failure being ( $I$ ) too advanced disease, in which case extraction is the only remedy ; (2) the choice of unsuitable applications, the cause of failure with some highly skilled and conscientious dentists; (3) insufficient persererance on the part of the operator or the patient, or of both.

I have now carefully watched for several years patients who have been methodically treated on these lines, and have done their duty by themselves and have observed a completely satisfactory result in at least 60 per cent. of the cases; when recurrence has occurred it has been slight and has soon yielded to a further short course of treatment, which is perhaps advisable in every case six months after the completion of the first.

Let us now consider the sccond of the above points. Despite all the study that has been devoted to the bacteriology of this disease, our knowledge stili remains in an unsatisfactory state; the reasons for this are several, among which may be mentioned:

(a) The obsession to find a "specific" microbe for every disease.

(b) The fact that far too little attention has been paid to the question of mixed infection, and that far too much attention has been paid to the results of cultural observations as opposed to the direct examination of stained smears. Many organisms associated with pathogenic conditions of the mouth are exceedingly difficult to grow, and cultural observations may prove entirely misleading.

(c) The lack of regard which has been paid to many organisms as possible causes of this pathological condition. As I have mentioned in another place, cultures of mouth spirochætes and spirilla are toxic in 
the extreme, yet little regard is paid to them as factors in producing either the local condition or the general constitutional symptoms. Vincent's organism is now well known to be capable of setting up an acute inflammatory condition, not only of the fauces but also of the tissues of the limbs, yet little importance is attached to this organism, even when present in enormous numbers in the pus of a pyorrhœic pocket.

The truth about the bacteriology of this condition I believe to be as follows: It corresponds exactly to that of other conditions about the mouth generally, and closely resembles, even if it is not actually identical with, that of follicular tonsillitis; it is also related to that of post-nasal catarrh, but less intimately. At the inception any one of these conditions may be initiated by a single variety of micro-organism, the pneumococcus, Streptococcus longus or brevis, staphylococcus (aureus certainly, albus possibly), and perhaps the $M$. catarralis. Secondary invasion by other organisms soon occurs, combinations of any of the preceding may be established, whilst before very long the spirochætes, spirilla, vibrios and other mouth bacteria also gain a footing, and may even in course of time completely oust the original invaders.

As these conditions are similar bacteriologically the absurdity of endeavouring to treat one of them locally and of neglecting the other is at once obvious; and in re-infection from another focus is found the explanation for many of the relapses which occur in cases of prorrhcea that have been apparently cured.

We are now in a better position to consider the question of vaccine treatment. Let us clearly realise that it is illogical and unwise in the extreme to consent to any shifting of responsibility. The dentist has his part, in my opinion the more important part, to play; the vaccine therapist cannot fill his role as understudy; if the dentist in attendance on the patient is not able or willing to deal with the infection locally in a thoroughly satisfactory way, then the physician should allow no scruples to stand in the way of advising that the help of a more able or more willing operator should be sought. Eight out of every ten of my pyorrhceic patients I have found to require no more than local treatment.

What scope is there, then, in this condition for raccine treatment? Local treatment may fail to effect a complete cure which the additional help afforded by raccine treatment may secure; in other instances cure may be expedited and speedy relief be afforded from some constitutional symptoms, and arrest be brought about in the case of others. The fairest way to state its claims to consideration is that it will rarely fail to yield some definite assistance to careful methodical local treatment, and will sometimes succeed in bringing about very material 
improvement, if not actual cure, in cases which obstinately refuse to yield to local treatment.

As I have said, the determination of the true bacteriology is no simple matter in any case. It will not by any means suffice to express pus from various pockets and culture it on agar or blood-agar and in broth. Special media may assist the growth of certain varieties of organism which will otherwise refuse to grow, but a conclusion as to the relative importance of the various bacteria seen in films and grown in cultures is best made only after a week or more of careful local treatment. In this way saprophytes and organisms merely resident on the surface of the diseased tissues are to a considerable extent eliminated; by means of a fine glass capillary pipette a little secretion is then removed from the bottom of several pockets, examined directly in smears and cultured on the selected media; a little scraping from the gum lining the pockets should be similarly treated. If growth be obtained of the organisms which direct examination of the secretion and infected tissues indicates as being likely to be concerned in the process, vaccines may be prepared therefrom. If thought desirable opsonic index determinations may be made towards the bacteria which have been isolated; personally I don't regard it as worth the time and money expended thereon. Suitable initial doses of the various organisms, perhaps, are streptococcus 5-10 millions, pneumococcus Io millions, staphylococcus roo millions, $M$. catarrhalis 25 millions, streptothrix Io millions, the interval between inoculations being five to seven days. Treatment may have to be continued for six or even twelve months, and repeated for short periods at intervals to guard against relapse.

\section{Results of Vaccine Treatment.}

A few abstracts from various sources will serve to show the divergent views taken as regards the ætiology of this disease and the benefits claimed for vaccines in its treatment. Williams (Amer. Journ. of Med. Sciences, May, IgII, p. 666) has reported his results in two small series of cases. The organisms which he held to be accountable were streptococcus, pneumococcus, $M$. catarrhalis, and staphylococcus, singly or grouped. Inasmuch as the only nutrient medium apparently employed was agar, the thoroughness of the bacteriological examination cannot be regarded as all that was to be desired. He claims that eight cases which received autogenous vaccines were intractable cases of long standing which had received careful dental treatment, while twelve other cases were dispensary ones which were treated with a stock vaccine made from a mixture of four of the above 
autogenous ones. Reaction to an inoculation was shown within one to two days by increase of sensitiveness and discomfort of the teeth, and once by swelling of a lymph-node on the floor of the mouth. I append short details of the cases treated with the autogenous vaccines.

CASE I.-Infection streptococcal. Four years' careful intermittent dental treatment had afforded some relief of symptoms, but this became of shorter and shorter duration. Eleven inoculations were given in thirteen weeks; the initial dosage was ro millions, the final go millions. After the third administration the patient was as free from symptoms as after any completed course of dental treatment. The ultimate result was' apparent cure, which was durable twenty months later.

CASE 2.-Duration of disease about five years: Streptococcal infection. Two years' active local treatment had produced great improvement but not cure. Two years after discontinuing local treatment there was a bad relapse. Three doses of 20, 30 and 40 millions of stock vaccine brought some improvement; local treatment was then recommenced, and seven doses of an autogenous vaccine of from $25-80$ millions were given at intervals of a week. Eighteen months later there was no apparent relapse.

CASE 3.-Streptococcal infection. Thirty teeth were badly involved and the general condition was very unsatisfactory. Fourteen inoculations of an autogenous vaccine were given in fifteen weeks, at the end of which time only one pocket was discharging, the others being apparently cured.

CASE 4.-Mixed streptococcal and staphylococcal infection. Nine inoculations were given in seven and a half weeks, the dosage of the streptococcal vaccine being increased from 6-6o millions, that of the staphylococcal being twice these amounts. After three months there was no relapse from an apparent cure.

CASE 5.-A very severe case of streptococcal infection. In thirty weeks eighteen inoculations were given in dosages of from IO-5O millions. All subjective symptoms disappeared and there was very great improvement of the pyorrhœea.

CASE 6.-Also a severe case of streptococcal infection. Thirteen inoculations of from 7-50 millions were given. Eight months after the last inoculation slight pus was found in one pocket.

CASE 7.-Again a severe streptococcal infection. Some teeth were so loose that they had to be extracted. Eighteen inoculations of 6-9o millions resulted in apparent cure.

CASE 8 was one of at least fifteen years' duration. Two inoculations of $\mathrm{I} 2$ and $\mathrm{I} 6$ millions of a stock streptococcal vaccine and two of autogenous vaccine were given. This resulted in considerable improve- 
ment. Treatment was then discontinued. At the end of three months the improvement was maintained.

Of the thirteen cases treated with stock vaccine, only four received more than six inoculations. Three of these seemed greatly improved, and a favourable opinion was formed of the results to be obtained in this class of case from the use of a stock vaccine.

MacWatters (Proc. Roy. Soc. Med., October, Igio, supplement, p. I72) relates his experience of forty-eight cases, of which thirty had completed treatment; this consisted in removal of tartar, the use of tooth-brush and carbolic powder before each meal, and a mouth-wash of 4 per cent. sodium chloride and $0^{\circ} 5$ per cent. sodium citrate to promote osmosis. No local applications were used. Streptococcal vaccines, autogenous when possible, were alone used, the initial dosage being 5 millions, repeated at intervals of eight days, and increased to Io millions as the lower dosages ceased to produce a reaction. $\mathrm{He}$ states that the effect upon such constitutional disturbances as dyspepsia morning vomiting, rheumatic pains and depression of spirits was very marked.

The thirty completed cases received an average of ten inoculations: of these twenty-one showed no return of pus two months after the cessation of treatment, while the other nine were greatly improved. The obvious comment upon these results is that a lapse of two months after the cessation of treatment is utterly inadequate for judgment upon the question of cure: complete absence of symptoms after six months will point to the probability of cure, and after twelve months may, perhaps, justify the application of this term, inasmuch as it must remain an unsettled point whether recurrence after this period of time is not entirely a matter of re-infection instead of a recrudescence of the old infection.

Eyre and Payne (Proc. Roy. Soc. Med., December, Igog, Odontological Section, p. 29), carefully studied the bacteriology in thirty-three advanced cases ; inasmuch, however, as they began these investigations, which were to guide them in treatment, with the preconceived idea, based on the animal experiments of Washbourn and Goadby with this organism, that the Streptococcus brevis is a harmless saprophyte, which it most certainly is not, a fallacy was introduced into their observations from the very beginning. In the thirty-three cases they assigned the rtiological role to the following organisms:

Staphylococcus aureus

M. catarrhalis

Streptococcus pyogenes longus

M. catarrialis and Streptococcus pyogencs longus Pneumococcus

2 cases.

9
7
I I , ,
$4 \quad$,


The Streptococcus brevis was present in all the cases, but its significance was disregarded; as, however, the term Streptococcus pyogenes longus was applied to all the streptococci which were pathogenic for animals some of their group may include forms which other observers would have classified as "brevis."

In twenty-six cases which were selected because either they were of great severity or refused to yield to local treatment autogenous vaccines were employed:

In 2 cases the ætiological factor was held to be Staphylococculs aureuls.

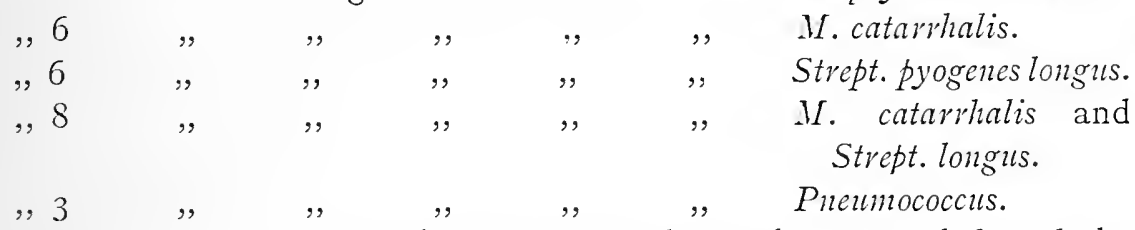

Before beginning vaccine treatment the teeth were scaled and the pockets packed on two occasions with some antiseptic, such as copper sulphate, tincture of iodine, a ro per cent. solution of formaldehyde, and a mildly antiseptic mouth-wash, such as $0^{\cdot} 2$ per cent. solution of formaldehyde or hydrogen peroxide 5-ro volumes. The doses of the various vaccines were-staphylococcal $50-250$ millions, $M$. catarrhalis $25^{-500}$ millions, streptococcus $5^{-250}$ millions, pneumococcus $5^{-100}$ millions, their later experiences leading them to employ the smaller dosages. Their intervals were seven to fourteen days, the number of administrations was from four to twenty-five, the average being six to twelve, and treatment was continued over periods ranging from one to nine months. "Cure" they regarded as being established when the teeth were firm, the mouth comfortable, mastication painless, no pus could be expressed, and the muscular and arthritic pains and digestive disturbances had disappeared.

They state that rapid improvement was noted in most cases, some being more resistant, and that the ætiological factor is of some importance in this connection. Infections by the staphylococcus respond most quickly, next those by the pneumococcus, next those by the streptococcus, then those by the M. catarralis, the most refractory being the double infections by the streptococcus and M. catarrhalis.

They claim to have examined the cases after considerable intervals and that in-

$\begin{array}{llrl}7 & \text { "cure" still persisted after I2-I5 months. } \\ \text { I2 } & ,, & , & 9-\mathrm{I} 2, \\ 2 & ,, & , & \text { under } 9,\end{array}$

Four were improved, I had died from malignant disease.

Three of the cases described as "cured" were shown at the 
meeting before which the paper was read, and the consensus of opinion among the dentists present was that the cases were not "cured," the condition being very similar to what might have been anticipated after local treatment only.

Goadby has been working at the bacteriology of this condition for many years and experience has compelled him frequently to modify his views, so that whereas he regarded the Streptococcus brevis as a harmless saprophyte in $\mathrm{r} 8 \mathrm{~g} 6$, he later came to regard it as the xtiological factor in a considerable percentage of cases. To a variety of the streptococcus which he prefers to call a strepto-bacillus, but which appears to be almost identical with the Streptococcus conglomeratus, so commonly present in the mouth and especially around and in the tonsils, he now assigns the chief rôle. For the results which he has obtained with various vaccines the original articles must be consulted (Proc. Roy. Soc. Med., February, Igro, Odontological Section, p. 55 ; Lancet, I909, vol. i, p. 663 ; ibid., I909, vol. ii, I875, etc.).

In the earliest stages he considers the prognosis to be 60 per cent. of cures, these cases apart from vaccine treatment being regarded as destined not only to advance of the pyorrhœa but to the diseases which have been shown to be associated with that condition.

The fallacy in this conclusion is that adequate local treatment of the disease at this stage, with proper supervision of the mouth at sixmonthly intervals and the inculcation upon the patient of the proper hygiene of the mouth, should result in roo per cent. of cures. A result of 60 per cent. of cures in this stage is synonymous with grave deficiences on the part of both dentist and patient.

Carmalt Jones, from the ubiquity of the Streptococcus brevis in the lesions, concludes that in the majority of cases this organism is the original cause of the condition, infection by other organisms being frequently superadded. He states (Therapentic Inoculation, p. II2) that twenty cases of simple pyorrhœa without complications have been treated at St. Mary's Hospital by vaccines of the Streptococcus brevis in doses varying from 10-50 millions, the usual initial dose being 20 millions. The results of treatment lasting from one to two months have been as follows : cured, six cases ; much better, seven cases; better, four cases; unchanged, three cases. Secondary infections by the $M$. catarrhalis in four cases, diphtheroid bacillus in one case and a coliform bacillus in one case were also dealt with. Distinct benefit to the complications was often noticed.

In summarising the results obtained by the various observers we find that good results are claimed alike by those who attach supreme rtiological significance to the ubiquitous Streptococcus brevis and by those who entirely disregard its claims; assuming for the sake of argu- 
ment that the clinical observations of all are equally reliable, the only reasonable conclusion that can be arrived at is that on the one hand we have those who, in the majority of their cases, are treating the primary infection and tend to neglect any secondary ones, and on the other hand we have those who pay exclusive attention to secondary or mixed infections and disregard the primary one. Some good is to be anticipated from either procedure and appears to be actually obtained. Of the sum total of good achieved, in my opinion the major portion is to be ascribed to the limited amount of local treatment which is also given, and to the fact that the patient's attention is more clearly focussed on the existence of the disease and on the ordinary hygienic measures which promote a healthier condition of the mouth.

A clearer conception of the true bacteriology of the complaint and of the prevalence of mixed infection would broaden the view that should be taken of the applicability of vaccine treatment and of the linesalong which it should be conducted.

\section{Conclusions.}

(I) The responsibility for the treatment of pyorrhcea alveolaris primarily rests with the dentist. Despite the great amount of attention which has been paid to this disease in recent years treatment is still frequently inefficient. Lack of thoroughness and of persistence and the use of improper local applications are perhaps the commonest cause of failure on the part of the dentist.

(2) Vaccine treatment cannot possibly alone suffice for cure; it should always be subordinated to careful adequate dental treatment.

(3) That it should be resorted to when the latter has already failed or appears to be likely to fail to effect a cure, or when such constitutional symptoms as chronic articular rheumatism, muscle pains, gastro-intestinal disturbances, or anæmia, simple or pernicious, require urgent attention.

(4) When the pyorrhœic condition is so advanced that extraction is the only course, and the extraction of one or two teeth is followed by violent constitutional disturbances, a few inoculations with the appropriate vaccine may greatly assist in raising the patient's resisting powers to the absorbed bacteria and their toxins, and enable the dentist to complete the extractions with less danger and discomfort to the patient.

(5) If vaccine treatment is to prove of any service adequate dosages must be employed and a prolonged course may be necessary. The signs of adequate dosage are the production of a definite but mild reaction, such as any of the following: slight malaise within eighteen hours; increase in local or general symptoms, as tenderness of the gums or teeth, joint or muscle pains; in default of these a definite improvement in the local 
condition showing no signs of relapse after five to six days. When the appropriate dosage has been found it should not be increased so long as steady progress is being made; when this ceases, or reactions fail any longer to be produced, then increase to double dosage may be safely made.

(6) When apparent cure has been brought about careful re-examination should be made at intervals of six months. In the event of relapse two or three visits to the dentist should be insisted on, and a short course of vaccine treatment recommenced.

(7) In those patients in whom constitutional symptoms are marked two or three inoculations every six months will probably assist in the maintenance of good health.

(8) Owing to the complexity of the bacteriology of this condition and the consequent difficulty in preparing the appropriate vaccine, failures in its vaccine treatment are especially apt to be experienced; in such instances the vaccine itself should come under suspicion and a reinvestigation of the bacteriology be made. The flora of the several pockets may vary greatly the one from the other, so that it is not sufficient to examine the pus from one or two foci and assume that a similar condition will be found in that from others. A member of the streptococcus group is the commonest causal organism, but it is not the only one, and mixed infections are not uncommon.

(9) Autogenous vaccines are much to be preferred to stock ones. The latter should only be used when insuperable difficulties stand in the way of the preparation of the autogenous.

\section{(d) Hay-fever.}

In this distressing complaint we have a condition of instability of the vaso-motor centre, and a great susceptibility of the mucous membranes of the eyes and nose especially to certain influences, the result being that the stimuli applied to the nerve-endings in the nasal mucosa upset the unstable balance of the vaso-motor centre; profuse lacrimation, reddening of the conjunctivæ, sneezing, swelling of the nasal mucosa, excessive formation of nasal, tracheal and bronchial mucus, and perhaps spasm of the unstriped muscle of the bronchial tubes with resultant asthma are thereby set up.

The stimuli may be of various kinds, such as certain perfumes, either of flowers, or of the products of incomplete combustion of petrol or other oils; more frequently it consists of a toxin, occasionally of bacterial origin but much more commonly, as Dunbar has shown, one derived from the pollen of various flowers and grasses.

Various lines of treatment may be therefore followed, such as:

(I) Stabilising the vaso-motor centre by cauterisation, electrical or 
chemical, of the nerve-endings in the nose. This method has yielded good results in certain hands.

(2) Removal of the patient from the reach of the stimuli-a course taken with many who leave England and Germany when the pollen is ripening, and depart to places such as Heligoland where there is no. pollen.

(3) Determination of the toxin to which the patient is susceptible and immunisation against it either by means of serum or vaccine. Dunbar, by inoculating horses with pollen, produced an anti-serum, which, while yielding good results in some cases during an attack, has, however, not proved very satisfactory in preventing the annual recurrence. Recourse has, therefore, been made to active immunisation by means of vaccine. As has been already mentioned, bacteria or their toxins are occasionally able to produce the condition. In two or three instances I have isolated the $B$. septus in pure culture from the nasal mucosa and secured complete immunity by inoculations with 250 and 500 million doses of the autogenous vaccine. I have also heard of one or two instances where the use of my combined vaccine for colds has produced a similar result. Carmalt Jones (Therapeutic Inoculation, p. I26) also secured considerable improvement in one patient by the use of an autogenous vaccine of the $B$. ac̈rogenes.

Cases such as these wherein a bacterial infection is the active agent must form a very small percentage of the total. The recent researches of Noon afford a certain method of determining whether susceptibility exists to pollen toxin; it is based upon the well-known ophthalmoreaction first applied to the diagnosis of tuberculosis, and the procedure is as follows:

One gramme of the pollen of Phlenm pratense is extracted with 50 c.c. of water, and for the sake of convenience the extract is arbitrarily assumed to contain 20,000 units of toxin in each c.c. The following dilutions are prepared from the extract : $5000, I_{500}, 500, I_{50}, 50, I_{5}$ and 5 units in each c.c., this wide range being necessary owing to the extreme variation exhibited by various individuals in their susceptibility to the toxin. Two or three drops of each dilution are put up in glass capillary tubes similar to those employed for the tuberculo-ophthalmic reaction. The test is applied in a precisely similar way; the patient sits with head thrown back, and the contents of a capillary tube of the 5-unit strength are instilled into the conjunctival sac of one eye, the other serving as control. After two or three minutes the eye is examined for a reaction; the first sign is usually a tickling at the inner canthus, which is very soon followed by a slight but distinct reddening of the caruncle; this constitutes a "slight" reaction. If the congestion extends to the adjacent conjunctiva it is called " marked," and if to the 
whole conjunctiva it is regarded as "very marked." If no reaction be obtained by the end of five minutes, the next stronger dilution, viz. the I5-unit strength, is instilled into the other eye and developments awaited. If no reaction is again obtained the next stronger dilution is employed, reverting to the eye first tested and so on until, if necessary, the strongest toxin has been used. If there is still no reaction immunity to the toxin of the pollen of this and closely allied grasses may be considered proved. If, however, a reaction is obtained with any of the dilutions, then susceptibility has been demonstrated. If a "slight" reaction be obtained with any strength then a "marked" reaction will be obtained with a triple strength, and a "very marked" reaction with a ten-fold strength.

Not only does this ophthalmo-test serve to demonstrate susceptibility, but it also indicates the suitable dosage for subcutaneous inoculation with a view to the production of immunity, as it has been observed clinically that the suitable dose for the latter purpose is one third of a cubic centimetre of the strength which yields the "slight" ophthalmo-reaction. Thus, if the latter be obtained with the r5o-unit strength, the suitable dose for inoculation is $\frac{1}{3}$ c.c. of this strength of toxin or I c.c. of the 50-unit strength. For purely protective purposes this dose should be repeated in eight to ten days. After a similar interval the next higher dosage may be employed, and so on, successful immunisation being shown to have been secured by failure to respond to the ophthalmo-test with the 5,00o-unit strength. During the hay-fever season it is well to begin treatment with half the dosage as above determined, the same rule holding in the event of the patient seeking relief from an actual attack; in this event not only should the dosage be halved, but the intervals between administrations should be likewise reduced; five days is then a suitable interval. Just as the application to the eye of two or three drops of the 500o-unit strength will indicate final immunity, so the failure to respond to the application five days after each inoculation of two or three drops of the strength triple that used for the last inoculation will serve to indicate that increased dosage is then advisable. The elaboration of this highly scientific method of treatment is of such recent date that it is hardly as yet possible to dogmatise about results. So far as one can judge from the experience of the past season, a considerable measure of success is to be anticipated. Many patients have escaped completely, and the greater majority have suffered from nothing but very mild attacks. With the adoption of a more highly polyvalent vaccine even better results may be secured.

Ellern (Deut. med. Woch., No. 34, I9I2) records an interesting series of observations made during the past season in Germany. He tested 
both the ophthalmo-reaction and the therapeutic effect of pollen toxin prepared at St. Mary's Hospital in a series of thirteen cases. He found that the concentrations required for obtaining the ophthalmo-reaction in his patients reached higher levels than in Freeman's observations; being that of $5^{\circ}$ units only in the most susceptible cases; the greater number showed subjective symptoms only with one of 500 units and distinct objective signs only with the I 500 units concentration. Healthy individuals were not affected by the jooo unit strength.

As to the relation of the ophthalmo-reaction to the therapeutic dose, he found that, at least during the period of prevalence of hay-fever, this was by no means constant, and that in some patients who felt subjective improvement in their symptoms the concentration required to produce the ocular reaction fell.

For therapeutic purposes he began with a dose corresponding to one fifth of the concentration required to produce the ophthalmo-reaction and repeated it at intervals of five days : higher initial dosage he does not consider desirable. No serious symptoms ever appeared as the result of inoculation and there was no rise of temperature; attacks of typical hay-fever of short duration occasionally occurred and once an asthmatic attack supervened. Doughy swelling with tension and pain at the site of inoculation frequently occurred, but these symptoms subsided in from one to two days.

The number of injections varied from + to 9-only few received less than 6 ; the dosage from 25 to 125 units as an initial and Ioo to 250 units (the latter in nine cases) as the final dosage.

The results were: unchanged 2, improved 5, markedly improved 6, as compared with attacks in previous years. The two unimproved cases gave up treatment prematurely.

Most of the patients declared their willingness to again undergo treatment next year, but all suffered from hay-fever for shorter or longer intervals. Ellern draws attention to the fact that in Germany hay-fever has been less severe this year than last; for instance, he made inquiry of twenty cases not treated specifically; their experience had been, 4 as last year, I + less severely attacked, and 2 very much less severe attacks. He therefore considers that the difference between the two series is too small to enable any definite conclusion to be formed, but admits that he may also have started the treatment too late in the season.

(e) Ozana or Atrophic Rhinitis.

Bacteriology.-Abel made a bacteriological examination of the atrophic nasal mucosa in Ioo cases of ozæna and found in each one of them a bacillus resembling, but not identical with, the bacillus of Friedlander. It is a non-motile capsulated Gram-negative bacillus which 
forms a colourless sticky growth on agar, which is so moist that it slides down to the bottom of the slope. In stab cultures it spreads out over the surface of the agar and does not form the nail-head growth characteristic of the bacillus of Friedlander. Its growth on gelatine is similar to that on agar and does not liquefy the medium.

It does not produce indol in peptone water.

Its fermentative properties are much less active than those of the bacillus of Friedlander. In two or three days it forms slight acid in litmus-milk, but no coagulum. It forms acid and gas in mannite, dextrose, maltose and raffinose within two days, in adonite and dextrin within three days, in saccharose, sorbite and lactose within four or five days, but not in dulcite or inulin at all. When this organism is injected subcutaneously into mice it proves fatal, when subcutaneously into guinea-pigs no abscess is formed, but if the peritoneal route be adopted death is brought about in about half the animals.

Abel found this organism in every case of atrophic rhinitis, but never in any other diseased condition of the nose. He employed it to inoculate a healthy human nasal mucosa and initiated an atrophic condition.

Vaccine treatment.-Page (Journ. Medical Research, July, I9I2, p. 489) found this organism in two cases of ozæna and employed vaccines of it in them for therapeutical parposes. In the first case inoculations were given every three days (no dosage is indicated). The patient noted continued subjective improvement, there was diminished crust-formation and increased secretion of mucus. After three months' treatment the improvement under this form of treatment alone was marked, but inasmuch as the right frontal sinus was involved cure had not then been produced, nor was this to be anticipated. The second case was one of twenty-five years' standing. In addition to the B.ozana, the $B$ pyocyaneus, streptococcus and Staphylococcus candidus were also present A mixed vaccine of the first two organisms was employed; after four inoculations at weekly intervals the fotor was diminished, the nose and throat not so dry, and the discharge was moister but less profuse. The treatment was being still continued. Cobb and Nagle (Amnals of Otology, Rhinology and Laryngology, St. Louis, 1912, vol. xxi) have also found vaccine treatment of distinct value in cases of atrophic rhinitis. I have myself found this ozæna bacillus in four cases of early atrophic rhinitis and employed autogenous vaccines, combined with another of streptococcus, pneumococcus, Staphylococcus aureus and M. catarrhalis in the respective cases. Initial doses of fifty millions were repeated or increased at intervals of seven days. The case wherein the Staphylococcus aureus was also present made very slow progress, and after six months' treatment was still not quite cured. The other three cases were cured 
after about this interval; final dosages of 500 and rooo million organisms were necessary in these instances.

\section{(f) Rhinoscleroma.}

Bacteriology. - Although the ætiology of this distressing complaint is still in doubt, there seems some justification for regarding it as a bacillary infection due probably to the bacillus of Frisch (vide " $Z$ ur Etiologie des Rhinoscleroms," Wien. med. Wochenschr., I882, Nr. 32, and also Kolle and Wassermann's Handbuch der pathogenen Mikro-organismen, Igo3, Bd. iii, pp. 4I4-424). This organism is a member of the bacillus of Friedlander group, but differs from others in appearing to be actively motile, and in retaining Gram's stain with some tenacity in sections of tissues hardened with Muller's fluid. Though the majority of experimental inoculations on animals with this organism have given a negative result, in a few instances typical scleromas have been produced.

Vaccine treatment.-Guntzer (Medical Record, July 24th, rgog, p. r29) has reported on two cases treated with vaccines after $X$-ray and other treatment had failed.

In the first case, where the nose, glottis and larynx were all affected, he gave three doses of $25^{\circ}$ millions of the autogenous vaccine at intervals of four and eight days; the last two of these injections produced marked local and general reactions, bloody discharge from the nose, malaise, headache, and rise of temperature. Subsequently doses of 375,500 and I000 millions were given at varying intervals, until the patient had received forty-three inoculations. At the end of this time, although cure had not been attained, the infiltrations had retrogressed, there was no longer dyspncea even on exertion, and the patient had been able to resume work. A year later, although treatment had been discontinued for some time, the improvement had not only been well maintained, but had been even continued.

The second case was a very advanced one, the general condition was bad, there was nodular deformity of the nose, the anterior nares. were almost closed, and there was infiltration of the upper lip; the tonsils and pillars of the fauces were grown together into a compact mass and nearly touched on opposite sides; the naso-pharynx was. almost obliterated, and the hard palate, but not the larynx, was involved. The vaccine was given in doses of $25^{\circ}$ to Iooo millions, at intervals of three to seven days, until thirty-two injections had been given. Local reaction was marked after each inoculation. At the time of the report the disease had not in any way decreased, but it had likewise not advanced, and the patient's general condition was very much improved. Treatment was to be continued. 


\section{THE MIAED INFECTIONS OF PULMONARY TUBERCU- LOSIS AND VACCINES IN THEIR TREATMENT.}

Out of the chaos into which the specific treatment of pulmonary tuberculosis had fallen a certain amount of order has now been evolved, but there is still much confusion and much misapprehension, and this it will be my endeavour to remove in this and the succeeding chapter. Difficulties have been created by the too ardent disciples of the various forms of treatment, who have tried to reduce man to a mathematical formula, and have based their treatment according to their solution of an algebraical problem. They have failed adequately to realise that they have insufficiently differentiated the signs and symptoms due to infection by the tuberculosis bacillus from those due to infection by allied invaders; more often than not they have ascribed to one invader what should have been attributed to another, with the inevitable result that the prescribed form of treatment has been misdirected and foredoomed to failure. They have also failed sufficiently to realise that the human being is a very highly specialised Individual, and that what applies to one individual may fail entirely to hold with regard to another. No scheme or system of treatment can therefore be evolved which will suit each and every phthisical person; the treatment must be moulded to the individual, not the individual to the treatment.

It will be my aim in this chapter to consider the first of these points in some detail, and to endeavour to demonstrate the paramount importance assumed by the allied or secondary invaders in many cases of pulmonary tuberculosis, and to indicate the means whereby this may be minimised or altogether removed and the case brought back to the much simpler form of an uncomplicated tuberculosis.

In the succeeding chapter I shall endeavour to consider how specific therapy can best be utilised in this simplified condition as well as in that of pulmonary tuberculosis as yet uncomplicated by the advent of secondary or allied invaders. 


\section{The Import of Mixed Infection.}

The term "mixed" infection is much to be preferred to that of "secondary" infection for the reason that certain bacterial diseases of the lung predispose to subsequent invasion of the pulmonary tissues by the $B$. tuberculosis, the latter infection being then the secondary one, the former the true primary one. In other instances the reverse holds true, to a primary invasion of the tissues by the $B$. tuberculosis secondary invaders become superadded. The ultimate result may be the same, but in the earlier stages the conditions are very different, and the methods of treatment may require to be very different. In the early stage in the first instance the problem is the prevention of the advent of the tubercle bacillus to pulmonary tissues which are in a condition called by French physicians "præ-tuberculous," and to remedy this præ-tuberculous state; in the latter instance the problem is to prevent the advent of other pathogenic bacteria to pulmonary tissues already invaded by the B.tuberculosis and to remedy this tuberculous condition.

In the later stages in both instances we have to deal with a co-existent infection by the $B$. tuberculosis and other pathogenic bacteria.

At the present time the most ridely divergent views are held as to the influence which other bacterial infections of the lungs exercise both upon the initiation of the tuberculous process and upon its subsequent course. Some authorities, basing their view upon the very fallacious observations that many of the organisms isolated from tuberculous sputum are of low vitality and of very little virulence towards animals, maintain that the part they play is a very subsidiary one-in the language of the stage, it is little more than a "walking-on" one. They point out that as ferer, wasting, caseation and softening may all, under certain conditions, be produced directly by the tubercle bacillus, the symptoms of the disease should in all cases be attributed to this bacillus, and to it alone.

Other authorities would attribute a preponderating influence to the other bacteria in the production of the advanced processes of this disease; others, again, have opinions of every intermediate grade between the two extremes, and I may perhaps be permitted to make the following quotations:

Powell and Hartley (Diseases of the Lungs, fifth edition, p. 402) write as follows: "For ourselves we cannot but believe that when such micro-organisms are present their action must be harmful, and that they at least prepare the way for the invasion of the tissues by the tubercle bacillus, and assist in that suppurative process which leads to the elimination of caseous products and manifests itself clinically by- 
the well-known phenomena of hectic. The matter requires further investigation, but for the present we cannot ignore the part played by secondary micro-organisms and must do all in our power to prevent their gaining access to the patient."

Sir A. E. Wright writes: "While the suggestion that mixed infections must be expected in the common suppurative processes which occur in connection with surfaces which harbour microbes may well be universally acceptable as not breaking in on any accepted ideas, the suggestion that mixed infection must therefore be considered in every case of phthisis, lupus, tubercular caries, tubercular cystitis and tubercular ulceration, in the very nature of things will be unacceptable to many clinicians. Such a suggestion will be felt to throw doubt not only on the clearness of vision of those who have sought for anti-tuberculous remedies in these diseases, but also on the critical acumen of those, who, without taking into account the fallacies which are incidental to clinical methods, have confidently undertaken to pass final judgment on anti-tuberculous remedies by the observation of their clinical effects in cases in which, in addition to the tubercle bacillus, other pathogenetic microbes may have been at work.

"Be it acceptable or unacceptable, there is no escape from the fact that practically every case of suppurating lupus is complicated by a staphylococcus infection and every aggravated case of lupus with a streptococcus infection. What holds true of lupus, mutatis mutandis is true of every tuberculous affection to which microbes can find access."

Foulerton (Transactions of the British Congress on Tuberculosis, London, I902, vol. iii, p. 6I2) points out that "an ox may have the most extensive tuberculous infection of the pleural or peritoneal sacs and yet will frequently be in absolutely prime condition; there is no suggestion of disease until the tuberculin test is applied or the animal is slaughtered. But, given a breaking-down of tubercles in the lung substance with secondary infection of the cavities or an ulceration of tuberculous lesions in the intestine, and one finds the same high temperature, the general wasting, and the same active destruction of the infected tissue which characterise the average case of chronic pulmonary phthisis in man."

Webb (Tuberculosis, Klebs, Igog, p. 599) writes as follows: "It is perhaps a fact that many of these secondary organisms are of low vitality and non-virulent, but it is just as impossible for such patients to rid themselves of these as of local infections, such as acne and furunculosis, both such frequent afflictions of the tuberculous. Surgeons, familiar with bone tuberculosis, know how well patients with pure tuberculous disease improve, and yet how intractable are those 
cases with mixed infection. Prudden's well-known experiments on rabbits show conclusively that the concurrent action of two distinct pathogenic germs may result in a considerable modification of the lesions which either could produce alone."

The question of mixed infection I propose to discuss according to the following scheme:

(I) Theoretical consideration of the question as to how far concomitant infection may be truly primary or truly secondary.

(2) The nature of the bacteria associated with the $B$. tuberculosis in cases of pulmonary phthisis.

(3) Method of determining the effect of the toxins of other bacteria upon the growth of the tubercle bacillus in vitro, results and theoretical deductions.

(4) Method of determining whether deductions from observations in vitro hold with equal force in the living'subject, and the application of the facts so learnt to the prevention and cure of mixed infections.

Theoretical Consideration of the Question as to how far Concomitant Infection may be truly Primary or trully Secondary.

The determination of the precise period of life at which invasion of the pulmonary tissues by the tubercle bacillus has occurred in any given person is a very difficult question. That infection of the glandular system occurs in a very high percentage of children in the first few years of life is an unquestioned fact. Some authorities maintain that in cities it is of almost universal occurrence. MacConkey and MacFadyen actually found virulent tubercle bacilli present, usually in the mesenteric glands, of about 25 per cent. of children who died from non-tuberculous causes.

The assumption that the pulmonary phthisis, which makes its appearance in considerably later years of life, is due to this infection contracted during childhood leads to many difficulties, such as that of explaining why the germs should lie latent, often over a period of many years, and then suddenly take on active growth. Also careful observations have tended to show that for about 50 per cent. of the cases of glandular tuberculosis in children, the bovine type of the tubercle bacillus is responsible; whereas in pulnonary phthisis the bacillus is found to be almost invariably of the human type. It thus follows that in 50 per cent. of the cases a metamorphosis of the bacillus in the direction of change of type must have occurred; and for the possibility of this occurrence there is little or no experimental evidence.

Reinfection by the human type bacillus must then have happened, 
and what is true for 50 per cent. of the cases is equally likely to be true for, at all events, a considerable proportion of the other 50 per cent.

Naegeli has found that in 96 per cent. of autopsies conducted upon bodies of between the age of eighteen and thirty years evidence of tuberculous infection was present, while in those above thirty none were found free. Infection by the tubercle bacillus is thus a practically universal occurrence, but in only a relatively small proportion of the cases do active pulmonary symptoms supervene. As to the interval of time that must elapse between the infection of the pulmonary tissues by the tubercle bacillus and the production of such symptoms as enable the detection of the process to be possible, no definite limits can be fixed. It varies much, probably with each individual, and may be a few weeks or a few years. The determination, therefore, of the question whether it was some other bacterial infection that predisposed the patient to infection by the tubercle bacillus and accelerated the process when infection had occurred, and if so, precisely what was its nature, is fraught with difficulty. As, however, I shall produce clear evidence of the effect of concomitant infection upon the growth of the tubercle bacillus, it is only reasonable to suppose that prior manuring, as it were, of the soil will conduce to subsequent invasion by the $B$. tuberculosis.

Clinical observations have shown that certain bacterial infections are frequently antecedent to the outbreak of symptoms of pulmonary tuberculosis. Thus whooping-cough and measles are antecedent to a large proportion of cases of tuberculosis in children; it is frequently to be observed that glandular enlargements in the neck are preceded by decayed teeth, a condition of pyorrhœea alveolaris, eruptions on the scalp, enlarged tonsils and adenoids, or by the sores set up by chickenpox, and that unless prompt treatment be directed against these disorders definite tuberculosis of the glands may ensue, and it is only logical to suppose that stimulation of the tubercle bacilli at other foci, such as the mediastinal and bronchial glands or in the pulmonary tissues, may simultaneously occur.

Pneumonia and neglected colds, again, enter into the history of a large proportion of cases of phthisis, and such importance do some French physicians attach to bronchitic and asthmatic conditions as predisposing causes of pulmonary phthisis that they are wont to call them præ-tuberculous conditions.

Whether in any given case some antecedent bacterial infection has been definitely responsible for invasion by the tubercle bacillus must, however, rest entirely upon pure inference. 
The Nature of the Bacteria associated with the B. Tuberculosis in cases of Pulmonary Phthisis.

I have, during the past four years, carefully and minutely examined the sputum of fifty-two cases of pulmonary tuberculosis with a view to the determination of the nature of any concomitant infection, with the following results :

\section{TABLE XIII.}

Total Number of Cases, 52.

\begin{tabular}{|c|c|c|c|c|c|c|c|c|}
\hline Streptococcus. & - & . & presen & 42 & times & $=$ & SI & er cent \\
\hline M. catarrhalis. & . & $\cdot$ & , & $4 \mathrm{I}$ & $\theta$ & $=$ & So & , \\
\hline Pneumococcus. & - & $\cdot$ & , & I9 & , & $=$ & 37 & , \\
\hline B. influenza & • & $\cdot$ & , & I2 & .. & $=$ & 23 & 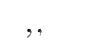 \\
\hline M. paratetragenus & . & $\cdot$ & ., & IO & , & $=$ & 20 & $\because$ \\
\hline Staphylococcus albus & & . & , & 8 & .. & $=$ & I5 & $\because$ \\
\hline Stapluylococcus aureus & & $\cdot$ & , & 6 & .. & $=$ & I2 & , \\
\hline Diphtheroid B. & . & . & $\because \cdot$ & 3 &. & $=$ & 6 & , \\
\hline B. of Friedlander & & . & , & 2 & ,. & $=$ & + & , \\
\hline B. proteus. & 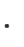 & 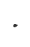 & , & 2 &. & $=$ & 4 & .. \\
\hline B. coli . & . & . & , , & I & , & $=$ & 2 & , \\
\hline
\end{tabular}

Only exceptionally was the mixed infection found to be monorganismal, this being more frequently the case with such organisms as the staphylococcus, B. of Friediander, or B. proteus, than with those which come above them in the above list; more often it was found to be binorganismal, in such combinations as B. influenza and pneumococcus, streptococcus and M. catarrhalis, pneumococcus and $M$. catarrhalis; and still more frequently the combination was a threefold one, such as $B$. influenza, pneumococcus and M. cataryhalis; pneumococcus, streptococcus and M. catarrhalis.

At times the precise character of the mixed infection would spontaneously vary from time to time, this being determined to a certain extent by the incidence of a general catarrhal epidemic among the populace; for instance, one case which I had carefully watched and repeatedly examined during two years without ever finding any concomitant infection other than that by the Staphylococcus albus, suddenly contracted a superadded infection by the $B$. influen:e and pneumococcus, with the unfortunate result that not only was the whole of the marked progress made during two years lost, but the process was transformed from a fibroid one into an acutely pneumonic one, and in less than a month I heard of the patient's death. 
During the past eight years I have had exceptional opportunities of making myself acquainted with the precise nature of the bacteria responsible for prevailing catarrhal epidemics, and while I do not think that phthisical cases are more disposed to catarrhal infection than other people, what I have certainly found to be the case is that there is a greater tendency for the bacteria to invade the structures in the chest instead of confining their activities to the upper respiratory tract, and that they cling there with greater pertinacity when once they have gained a hold. It thus follows that one or two examinations of the sputum of a phthisical case by no means suffice to determine what is the nature of the true accessory infection-by that I mean the infecting organism which is always present irrespective of the fact whether a general catarrhal epidemic may be in progress or not; even the minute observations which I have bestowed on several cases have not sufficed to enable me to determine what was the true primary accessory infection. The methods which I have elaborated, however, enable us to sort out the bacteria present at a given time, and say which are exerting a malignant influence and which apparently are not.

It is due to causes such as the above that lists by various observers, attempting to show the relative frequency with which the various bacteria occur as complicating agents in cases of pulmonary phthisis, show such considerable variations.

It does not become me to belittle the work of other investigators, but I have already pointed out the extreme care with which observations upon the bacteriology of chest infections must be conducted; in the case of some it is easy to determine that sufficient care has not been so bestowed; with others sufficient details to estimate the probable accuracy of their observations are not available. It thus becomes a difficult, nay, an impossible, matter to collate the various records and draw up anything like an accurate table to display the relative frequency with which the various bacteria play the part of secondary invaders to the $B$. tuberculosis. At the same time there is a considerable degree of concordance in the findings as regards the frequency with which the streptococcus, $M$. catarrhalis, pneumococcus and staphylococcus are found; for instance, this is the exact order of frequency with which they were found by Dr. T. W. Hastings (quoted in Kleb's Tuberculosis, p. 59r) as well as by myself. Other observers have found the Staphylococcus (albus or aureus) of practically universal occurrence, but this I feel is due either to lack of care in excluding contamination from the mouth and pharynx, or to the observations having been conducted upon cases in very advanced stages of the disease, for although I cannot offer figures in support of my view, I yet feel strongly that the earlier the stage of the disease, the less the likelihood of invasion of the 
tuberculous foci by the more purely pyogenic bacteria, and the greater the probability of the more purely catarrhal organisms complicating the infection.

The results of post-mortem examinations such as are recorded by numerous observers I think should be altogether excluded from consideration, for it is impossible to exclude agonal and post-mortem infection, and even granting that such had not occurred, observations derived from so far advanced cases are not of practical application to cases in the early stages, and these are those with which we are the more concerned.

The frequency with which some observers have found the $B$. coli communis I am altogether unable to explain; personally I have only once encountered it in cases of pulmonary phthisis and only in one case out of several of lung abscess, that particular case having a very doubtful history of an early tuberculous lesion several years before the onset of the abscess at a quite different place.

In cases of basal phthisis complication of the infection by the $B$. coli would be more likely to occur than when the tuberculous process is confined to the upper lobes.

The value of the precise determination of the frequency with which the various bacteria play the rôle of accessory invaders in phthisis would, as I have already hinted, be much increased if more regard were also paid to the record of the stages of the disease in which they are found; to this, personally, I intend in future to pay more heed, for the bearing upon the question of prophylaxis is obviously a most important one. If, as I believe, the catarrhal organisms, M. catarrialis, pneumococcus, B. influenza, M. paratetragenus and some varieties (nonpyogenic as a rule) of the streptococcus, show a far greater predilection for early cases than do the more purely pyogenic organisms which appear to follow in their tract, measures for preserving, not only early phthisical cases, but also those who merely seem to be predisposed to pulmonary phthisis, from catarrhal attacks assume a yet greater importance.

Method of Determining the Effect of the Toxins of other Bacteria upon the Growth of the Tubercle Bacillus "in vitro"; Results and Theoretical Deductions.

During my investigations into the bacteriology of the catarrhal diseases of the respiratory tract, I have been frequently struck by the observation that in certain cases of almost pure infection by the $B$. infunenze this organism refused to grow on the culture plates unless, perchance, development also occurred of a few colonies of such organisms 
as the staphylococcus, pneumococcus, or $M$. catarrhalis. Luxuriant growth of the $B$. influenza would then occur in immediate proximity to these colonies, but not elsewhere.

It occurred to me to study this symbiosis further, and I found that it sufficed to add to the melted blood agar 2 or 3 c.c. of a killed broth culture of these various bacteria prior to pouring the plates and inseminating them with the B. influenza. By this device I have obtained growths of this organism so profuse that they looked more like cultures of the $B$. coli than of the influenza bacillus. Previously I had recorded in the Lancet the extraordinary inhibitory effect that the bacillus of Friedlander exerted upon the growth of other microorganisms in, or on, the same medium.

The thought then suggested itself-I wonder what is the effect upon the growth of the tubercle bacillus that is produced by the simultaneous growth of the other such bacteria as occur in secondary infections of pulmonary phthisis, or by their toxins. The inability to isolate the tubercle bacilli from my cases was at first an obstacle to the solution of this question, but this fortunately was removed by the discovery of the antiformin method of securing pure cultures of the tubercle bacillus.

By the methods already outlined I proceeded to prepare pure cultures of the tubercle bacillus and of all the accessory microbes from several of my cases. Subcultures of the accessory microbes were then prepared in suitable fluid media-broth for staphylococci, bloodbroth for the B. influenza and pneumococcus, serum-broth for the $M$. catarrhalis-and these allowed to incubate at $37^{\circ} \mathrm{C}$. until such time as full development of any toxin they might form had taken placeusually three to six weeks-by which time free growth of the tubercle bacillus upon the Dorset's egg medium had also occurred.

Tubes of the same media without prior insemination with any bacterium were also placed in the incubator to incubate along with the other tubes, and served as controls in the way which will be indicated.

The fluid cultures were then sterilised by heating for one hour at a temperature of $65^{\circ}-70^{\circ} \mathrm{C}$. The control tubes were similarly treated. A sufficiency of large tubes of Dorset's egg medium was then prepared from the same egg-mass and inspissated and sterilised together, so that there should be no doubt at all events of the initial precise similarity of the soil upon which future growths were to be obtained. Care was taken that exactly equal amounts of medium were added to every tube, and that the angle of slope in the inspissator was the same for all; the tubes being the same size, the area of the surface of the medium should be the same; if measurement showed that some failed in this direction they were not utilised for the purposes of these experiments. 


\section{PLATE IX.}

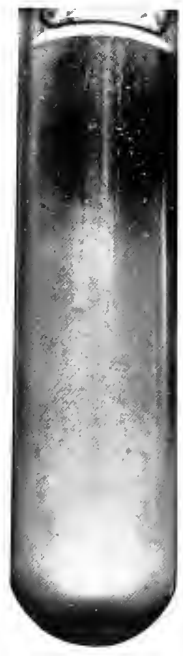

FIG. 39" - -No toxin. Five weeks' growth.

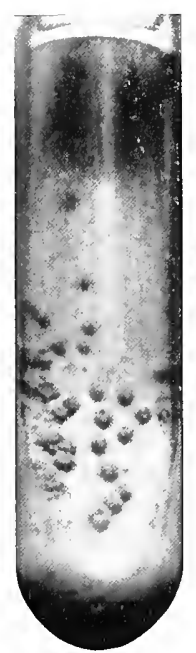

FIg, 39 --Pneumocorcal toxin. Five weeks' growth.

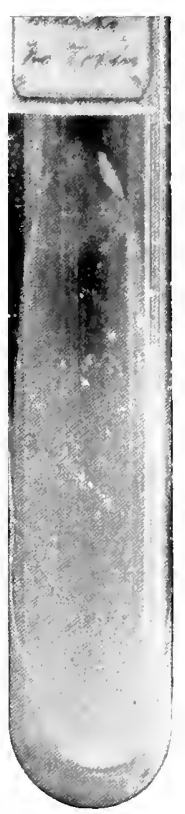

FIG. 40". - No toxin. Five weeks' growth.

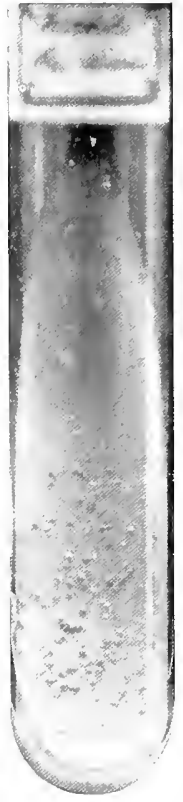

Fig. 40". -Auto-pneumotoxin. Five weeks' growth.

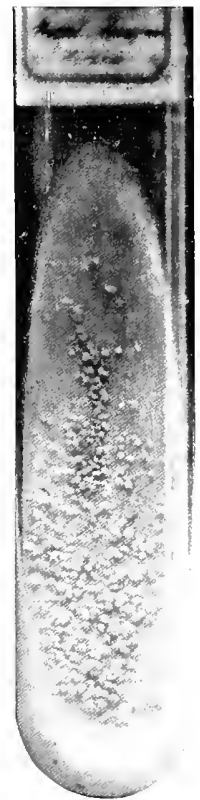

FIs. 40.-Auto-streptococcus toxin. Five weeks' growth. 

That number of tubes of the finished medium which corresponded to the number of cultures of the accessory microbes plus the same number to act as controls were taken and all water of condensation pipetted off from them. They were then treated as follows:

To tube $\mathrm{I}^{\mathrm{b}} 2$ c.c. of the sterile toxin, say of the pneumococcus, was added;

To tube $I^{a} 2$ c.c. of the fluid from the control tube corresponding to the pneumococcal one;

To tube $\mathrm{II}^{\mathrm{b}} 2$ c.c. of the sterile toxin, say of the B. infuenzar;

To tube $I^{\mathrm{a}} 2$ c.c. of the fluid from the control tube corresponding to the B. influenza one;

and so on, the $\left(^{a}\right)$ tubes always serving as controls.

These various tubes were then lightly plugged and sloped so that the added fluid bathed the whole surface of the egg medium; they were set aside in the incubator at $37^{\circ} \mathrm{C}$. until such time as the surface of the medium was moist, but nothing more. This occupied two or three days. From the growth of the tubercle bacillus, which had been isolated from that same case, an emulsion in I per cent. salt solution was then prepared and centrifugalised at very high speed for several minutes; the upper portion was pipetted off and examined for the presence of any clumps; if these were present further centrifugalisation was performed until a thin, perfectly uniform emulsion had been ensured. By means of a very fine sterile glass capillary pipette exactly equal volumes of the emulsion of tubercle bacilli were added to each of the above egg-medium tubes and very carefully distributed over the surface of each. The tubes were then sloped and placed in the incubator for forty-eight hours, when they were re-plugged with dry sterile woollen plugs, covered with a rubber cap, returned to the incubator in an upright position and allowed to incubate. At the end of three to five weeks the appearances of the various tubes were found in certain instances to differ in the most striking way.

Some of the results are shown in figs. $39-42$, Plates IX and X.

For instance, figs. $39^{\mathrm{a}}$ and $39^{\mathrm{l}}$, Case (I), show the different growths obtained after five weeks' incubation upon two tubes of Dorset's egg medium, the former having been manured with 2 c.c. of blood broth, the latter with 2 c.c. of blood broth in which a pneumococcus, isolated from the same case as that from which the tubercle bacillus had been derived, had been allowed to elaborate its toxin. The acceleration in rate of growth of the tubercle bacillus produced by the pneumococcal toxin must roughly be million-fold. The specimen of sputum was sent to me by Dr. Roemisch, of the Wald Sanatorium, Arosa; it came, I believe, from a rather early and subacute case which was not doing well. 
Figs. $40^{\mathrm{a}}, 40^{\mathrm{b}}$ and $40^{\mathrm{c}}$ (Case 2) illustrate the results obtained from a case in which the left upper lobe was little more than a mere shell surrounding a huge cavity, the left lower lobe being in a condition of almost complete consolidation; there was a very doubtful focus of infection at the right apex; a chain of enlarged glands ran down the right side of the neck. Dr. Rufenacht Walters kindly confirmed my clinical observations. Two organisms, a pneumococcus and a Streptococcus longus, were isolated from the sputum. The effect their respective toxins produced upon the growth of the concomitant $B$. tuberculosis is well seen in the preparations; it will be noticed that the acceleration produced by the toxin of the Streptococcus longus was greater than that produced by the toxin of the pneumococcus.

Figs. $4 \mathrm{I}^{\mathrm{a}}$ and $4 \mathrm{I}^{\mathrm{b}}$ (Case 3) illustrate what occurred in the instance of a case of chronic phthisis. The only accessory microbe seen in smears and isolated in cultures was a peculiar diphtheroid, not Bacillus septus. It will be noticed that the growth in the manured tube was in no respect better than that in the unmanured one ; and it may therefore be deduced that if this diphtheroid organism did elaborate a toxin, this latter was without influence on the rate of growth of the allied B. tuberculosis.

Case 4 , iliustrated by means of figs. $42^{\mathrm{a}}, 42^{\mathrm{l}} 42^{\mathrm{c}}$, and $42^{\mathrm{d}}$, was one of very chronic and slowly progressive tuberculosis of both apices, and of advanced but very slowly progressive laryngeal phthisis. Repeated examinations extended over a period of two years failed to reveal the presence of any accessory microbe other than the Staphylococcus albus.

Figs $42^{\mathrm{a}}$ and $42^{\mathrm{b}}$ show the respective growths obtained after eight weeks' incubation upon an unmanured soil in the first tube and upon one manured with the Staphylococcus albus toxin in the second; they will be seen to be practically identical. These observations were repeated several times, always with the same result; if anything, there was a shade better growth upon the unmanured medium than upon the manured one. The significance of this will be considered later.

Other experiments equally clearly showed that the toxins of certain bacteria had a very marked influence upon the rate of growth in artificial cultures of the tubercle bacillus associated with them in any given case of phthisis. It therefore remained to ascertain whether in those cases in which no such accelerating effect was evidenced the failure was due-

(I) To an inability on the part of that particular strain of tubercle bacillus to respond to the stimulus of the toxin, $i$.e. whether the fault lay with the B. tuberculosis.

(2) To an inability on the part of that particular toxin to stimulate 


\section{PLATE $X$.}

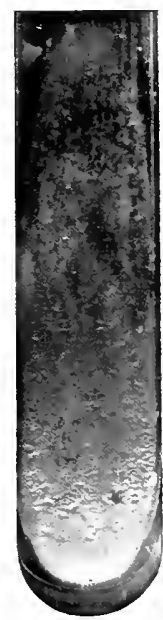

Flg. 4I ${ }^{a}$.-No toxin. Five weeks' growth.

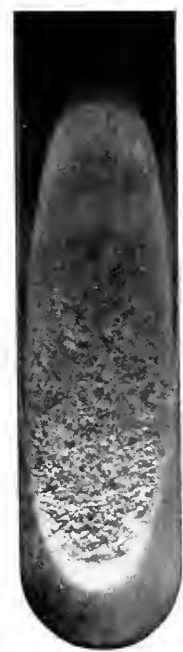

FIG. 42. -Anto-staphylococcus toxin. Six weeks' growth.

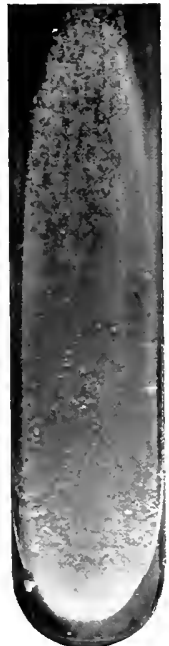

F1G. $41^{b}$.- Auto-bacillary toxin. Five weeks' growth.

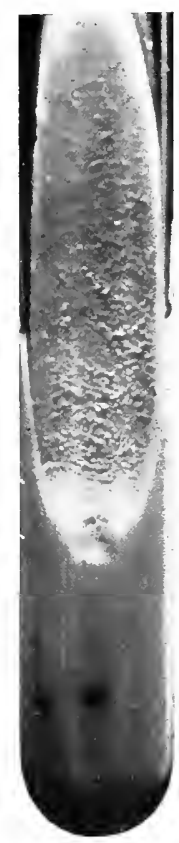

FIG. 42 $2^{c}$.- Hetero-pneumotoxin. Six weeks' growth.

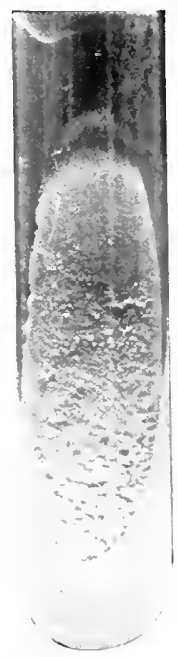

Fig. $+2^{\prime \prime}$.-No toxin. Six weeks' growth.

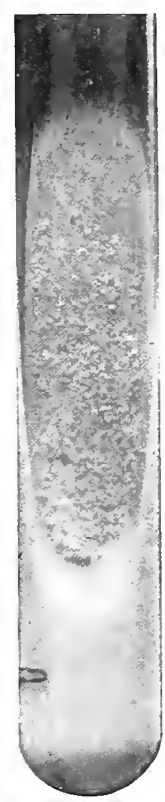

FIG. 42.-Hetero-streptotoxin. Six weeks' growth. 

the growth of the tubercle bacillus, i.e. whether the fault lay with the toxin.

If the former of these hypotheses were the correct one, then it should not be possible to stimulate the growth of that particular $B$. tuberculosis by manuring the soil with any other toxins of proved powers of stimulation derived from allied organisms in other cases of tuberculosis. Accordingly some of the pneumococcal toxin from Case I (above) and some streptococcal toxin from Case II (above) were employed to manure the soil of two culture-tubes. These experiments were conducted side by side with those already detailed as $42^{\mathrm{a}}$ and $42^{\mathrm{b}}$, the same control, $42^{\mathrm{a}}$, sufficing; here also no acceleration in the rate of growth of this strain (IV) of the $B$. tuberculosis was produced, $c f$. figs. $42^{\mathrm{a}}, 42^{\mathrm{c}}, 42^{\mathrm{d}}$, and inasmuch as repetition on several occasions with other toxins produced the same result, the deduction appeared to be justified that this particular strain of the $B$. tuberculosis was incapable of stimulation in this manner, at all events by one streptococcal, one influenzal, two pneumococcal and one staphylococcal toxin.

If the latter hypothesis were correct then the staphylococcal toxin should prove incapable of stimulating the rate of growth of strains of the $B$. tuberculosis derived from other sources. Experiment showed clearly, however, that it did possess this power towards an alien strain of the B.tuberculosis, and justified the conclusion that in this particular strain of the $B$. tuberculosis, there was an inherent lack of power to respond by increased rate of growth to the stimulus afforded by the perfectly efficient toxin liberated by the concomitant invader, and also to that afforded by such other active toxins as were investigated.

From these experiments it follows :

(I) That there are certain strains of tubercle bacilli, whose rate of growth, in vitro, can be accelerated by the aid of the toxins of a concomitant invader.

(2) That there are certain strains which cannot be so acted on by toxins derived either from a concomitant invader or from a heterologous source.

(3) That there are toxins elaborated by certain strains of bacteria which have the power of accelerating the rate of growth of the tubercle bacillus obtained from a concomitant infection.

(4) That there are toxins which fail to exercise this power upon the tubercle bacilli derived from the same source, and yet are perfectly efficient towards other strains of the B. tuberculosis.

It remained to investigate the following questions:

(a) Are the strains of tubercle bacillus falling under (I) above likewise capable of stimulation by the toxins of heterologous bacteria, and if so, of what bacteria? 
(b) Do the toxins which possess the power described under (3) also influence alien strains of the tubercle bacillus in a similar way?

(c) Are there toxins (if such be formed by the bacilli) which fail to stimulate both the allied $B$. tuberculosis and alien strains as well.

(d) Will the filtered products of bacterial growth act in the same way as the killed suspensions of the bacteria in their culture media.

To question $(a)$ numerous experiments enable me to reply that a strain of tubercle bacillus capable of stimulation by the toxins of associated bacteria is also capable of stimulation by the toxins of bacteria isolated from other cases of pulmonary phthisis, the degree of stimulation given varying, however, with different organisms and with different strains of the same organism; with some the acceleration of growth is very great, with others it is very slight. Roughly, the bacteria may be arranged as follows in regard to their accelerating powers, those with the greatest power being placed first:
Streptococcus.
I. paratetragenus.
Pneumococcus.
Staphylococcus aureus.
B. influenza.
B. diphtheria.
M. catarrhalis.
Staphylococcus albus.
B. coli.

The experiments which enable me to give an affirmative answer to question $(a)$ likewise afford an answer in the positive to question (b). To question (c) I am not yet in a position to give an answer in either direction.

Question (d) I have fully investigated for all bacteria associated with pulmonary phthisis which are considered not to form extra-cellular toxins, and have found that cultures of bacteria, even of many weeks growth, fail to produce any acceleration upon the rate of growth of the tubercle bacillus if these cultures are sterilised by filtration through Chamberland candles instead of by the aid of heat. The presence of the bacterial elements seems essential for the production of the effect.

Assuming that these bacterial toxins are able to exercise the same influence in the human body as they do under artificial conditions-and in support of this hypothesis I will adduce certain evidence-it then follows that the mixed infections in cases of pulmonary phthisis may play a two-fold part, viz.: (I) that of exerting an accelerating influence upon the rate of multiplication of the B. tuberculosis, and (2) that of producing the results which we have learnt to associate with their presence in other parts of the body, each variety of bacterium causing changes peculiar to itself. For instance, the staphylococcus is known to elaborate three substances: (I) a leucocidal substance, giving rise to cell necrosis and liquefaction of the tissues; (2) an endotoxin, whose liberation on the death of the cocci produces a general as well as a 
local toxic effect; (3) a hæmolysin, to which the body almost always contains an ample antibody, so that anæmia is not a striking characteristic of staphylococcal infection. The deduction may fairly then be made that a complicating staphylococcal infection must play some part in cavity formation and in the production of toxic symptoms; as, however, the staphylotoxin is an endotoxin and only liberated by the death and lysis of the bacteria, the toxic effect should be a limited one. This, in practice, we find to be the case ; high pyrexia is only exceptionally found in cases in which pulmonary tuberculosis is complicated by a pure staphylococcal infection.

Again, the pneumococcus appears to form not only a leucocidin and a powerful endotoxin, but also possibly some little exotoxin; inasmuch as the leucocidin is certainly not so powerful as that of the staphylococcus, while the toxins are considerably more potent, it might be anticipated that with a concomitant pneumococcal infection cavitation effects would be rather less marked and toxic effects more marked than with the staphylococcus; this appears to be the case.

Again, as the Micrococcus catarrhalis appears to give rise to no leucocidal substance and probably to no exotoxin, the part it can play in the causation of cavitation and pyrexial symptoms must, therefore, be a small one; its energies are devoted to the production of catarrhal symptoms; its power of stimulating the rate of growth of the tubercle bacillus, as judged from certain test-tube experiments, would appear to be considerable.

Finally the B. influenza would appear to form little or no leucocidin, but considerable quantities of a very potent endotoxin; whether it forms exotoxin is not known. Its influence in the production of cavities should, therefore, be small, but in the production of pyrexial symptoms great. Some confirmation of this is afforded by clinical observations.

Inasmuch as we have learnt from the preceding experiments that the presence of a concomitant infection does not necessarily mean the exercise of an accelerating influence upon the growth of the $B$. tuberculosis, either owing to the fact that the micro-organism in question is incapable of elaborating such a stimulin altogether, or because the stimulin it does form is ineffective towards the particular strain of the $B$. tuberculosis with which it is associated, or finally because the latter is incapable of stimulation by the toxin of any other micro-organism, and inasmuch, moreover, as the action peculiar to the associated microorganism may by no means necessarily be for evil-for instance, the lytic action of the staphylococcus may not necessarily be prejudicial to the patient, but, on the contrary, may lead to softening of the foci, the voiding of vast numbers of the tubercle bacillus, and ultimate 
healing of the cavity so formed-it necessarily follows that when a concomitant infection is present the precise rôle played by each of the allied invaders should be accurately determined. Every case of pulmonary tuberculosis is a complete law unto itself, and generalised deductions are, therefore, fraught with danger.

Careful observation has convinced me that in the most rapid cases of pulmonary phthisis, such as the miliary and broncho-pneumonic, mixed infections may be altogether absent, or the numbers of the associated micro-organisms relatively to those of the $B$. tuberculosis may be extremely small. It does not, however, necessarily follow that the extremely rapid multiplication of the tubercle bacilli is not due to a prior manuring of the soil, say with the toxins of the pneumococcus or $B$. influenza in the first case, or that the supply of manure furnished by the few concomitant invaders in the second place is altogether insufficient or is ineffectual in producing an ill-effect.

I have the opportunity of determining the accelerating effect in vitro of the secondary invaders upon the B. tuberculosis from one case only of acute broncho-pneumonic phthisis. The experiments were not altogether conclusive, but they appeared to show that the toxins of the associated micro-organisms produced no acceleration on the growth of the associated tubercle bacillus.

The numbers of the secondary invaders relatively to those of the tubercle bacillus in specimens of sputum voided from this case were extremely small from the inception of the infection to the very end.

On the other hand, in the case illustrated by figs. $39^{\text {a }}$ and $39^{\text {b }}$, the numbers of the pneumococci in the sputum relatively to those of the $B$. tuberculosis were also very small, yet we see how great was the influence of the toxin of the former upon the rate of multiplication of the latter.

We therefore learn if these cultural experiments have any definite value - and this I hope to show is certainly the case-that actual numbers of secondary invaders appearing in the sputum have little bearing upon the future progress of the case. Their true importance may be estimated by cultural experiments and observations upon the patient in the manner which I shall now describe.

Method of Determining whether the Deductions made from Cultural Observations have any Value when applied to the Human Subject, and the Application of the facts so Learnt to the Prevention and Cure of concomitant Infection.

If the observations made in vitro hold with equal force in regard to the human body, it should obviously follow that if a concomitant infec- 
tion can be stamped out not only should such symptoms as are directly referable to the secondary organism or organisms be caused to disappear, but also a definite effect should, in most instances, be produced in course of time upon the growth of the tubercle bacillus itself.

The question at once arises in any given case of phthisis, is it possible to determine the precise rôle that is being played by allied invaders and the tubercle bacillus respectively? While I am not prepared to state that this can always be accomplished, in many cases it most certainly can be done.

The method depends largely upon the most careful stethoscopic examination of the chest and the detailed observation of the change in physical signs produced by the inoculation of therapeutic doses of autogenous vaccines and of tuberculin respectively. Inasmuch as the determination of the precise nature of the concomitant infection is not a very difficult matter, the task before one would be greatly simplified if the exact nature of the physical signs which may be produced in the chest by each micro-organism were known; this, unfortunately, is not the case.

That all the physical signs producible by the B. tuberculosis + a concomitant infection, with the single exception perhaps of those of cavitation, may be produced by a perfectly pure infection by the $B$. tuberculosis, I believe to be the case, but none the less is it true in the great majority of instances that by far the greater proportion of the physical signs which most clinicians would assign to the action of the $B$. tuberculosis are in reality due to the other micro-organisms present.

The precise part played by the $B$. tuberculosis and by the allied organisms respectively is determined in the following way.

The patient is confined to bed under the most favourable conditions that can be secured; the temperature and pulse are recorded fourhourly, and the daily amount of sputum measured. Specimens of the sputum are examined in the ways already indicated, cultures made of the $B$. tuberculosis and of all the secondary invaders, vaccines and toxin preparations of each of the latter prepared, and in due course of time the accelerating effect of the toxin of each associated micro-organism observed.

Daily stethoscopic examinations of the chest are also made and the observations recorded upon a suitable chart; the best form for this purpose is the diagrammatic one employed here, as this affords ample room for the insertion of such signs as we may employ. When the patient has settled down into a more or less constant condition as regards pulse, temperature, physical signs and expectoration, by which time vaccines should have been prepared, we are ready to proceed. Obviously 
there are two possible ways of beginning: (I) by employing tuberculin inoculations and observing their effects; (2) by employing the vaccines either singly or in combination and observing their effects. The tuberculin and vaccines should never be employed together as the effect of one will completely mask that of the other. Personally I think the best procedure is to estimate the effect of the vaccine before that of the tuberculin and to employ the vaccine of one organism at a time, in order that the precise influence of each may be determined. To this, however, there is one practical objection to which reference will be made later.

Accordingly the physical signs in the chest having been again recorded a therapeutic inoculation of the vaccine is performed, such dosages as the following being employed: (I) B. influenza, roo millions; (2) Staphylococcus, Ioo millions; (3) B. septus or other diphtheroid, roo millions; (4) M. catarrhalis, 50 millions; (5) M. paratetragemus, 50 millions; (6) B. of Friedlander, 50 millions ; (7) B. proteus, 50 millions ; (8) Streptococcus, 25 millions; (9) pneumococcus, 25 millions; (Io) B. coli, 25 millions.

As a second examination of the chest should be made in about twelve hours it is most convenient to select an hour between $\delta$ and Io p.m. for the inoculation. The records of temperature and pulse are of course continued, and the sputum measured at first at twelve-hourly intervals, later at daily ones. Subsequent observations then of the chest are made after the lapse of twelve, twenty-four and forty-eight hours and daily thereafter.

If the organism of which the vaccine was employed be concerned in the production of signs and symptoms and the dosage employed be adequate, a definite effect should be produced in any one or more of the following directions within twelve hours:

(I) There may be rise in the pulse-rate of I0-20 beats;

(2) there may be rise in the temperature of $0.5-5^{\circ} 5^{\circ} \mathrm{F}$.;

(3) there may be an increase in the quantity of sputum;

(4) there may be an increase or alteration in the physical signs; and of these the last is the most important.

Comparison of Charts $15^{a}$ and $15^{b}$ will serve to illustrate the actual changes so produced in a certain case.

Fresh examination at the end of twenty-four hours should show a swing back in the condition of the patient:

(I) The pulse-rate should be the normal of the patient;

(2) the temperature should have fallen to its old level or to a lower one ;

(3) the sputum may be diminishing;

(4) the physical signs should be improving, and may correspond to 
what they were before the inoculation or a certain amelioration may have been produced (Chart $I 5^{c}$ ).

At forty-eight hours the changes noted after twenty-four hours should have advanced further in the same direction (Chart $15^{d}$ ). In some instances there is a somewhat delayed reaction : the changes which are described above as being typical of the twelfth hour hare not been evidenced till the twenty-fourth hour, those of the twenty-fourth hour not being eridenced till the forty-eighth hour. By the end of seventy-two hours there may be a further improvement in the clinical signs of the patient and this may continue for several days. A time will, however, come, largely dependent on the dose of vaccine given, when instead of improvement a retrogression will be initiated, this is the signal for a repetition of the raccine.

Front of Chest

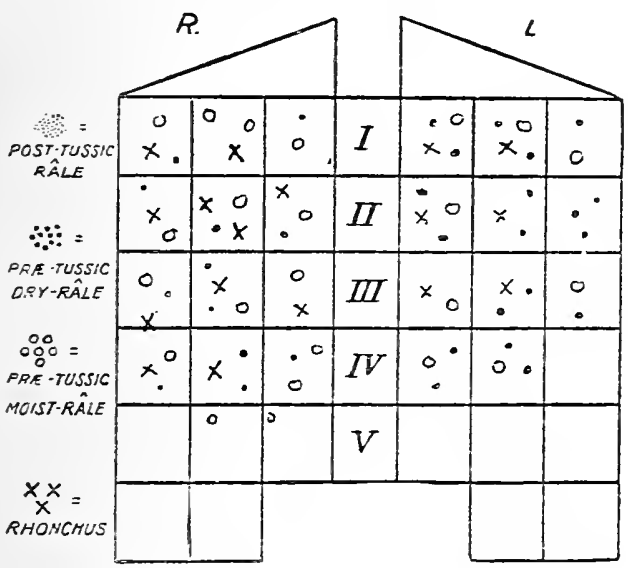

CHART $15^{n}$.-Before first inoculation.
Back of Chest

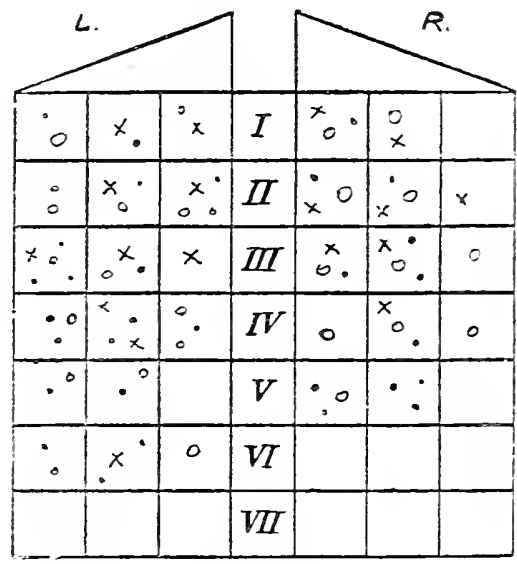

Sputum $=6$ oz. per day.

Occasionally it will happen that no reaction whatsoever is obtained; this indicates one of two things, either $(a)$ that the dose was too small, or (b) that the vaccine itself has no porrer of stimulating the formation of immune bodies. If the former aiternative be thought likely a double dose is given at the expiration of seventy-two hours and observations continued as before: this, I think, should always be done before assuming the latter alternative, $(b)$ that the vaccine itself is useless, which indicates that the corresponding organisms are producing no effects at the infected foci, and that trial of a vaccine of another of the organisms should now be made.

If this procedure be carefully carried out it will be found that in those cases in which the secondary infection is a multiple one, there is a marked difference in the response which is made to inoculations of the sereral vaccines; that some produce much more effect upon the 


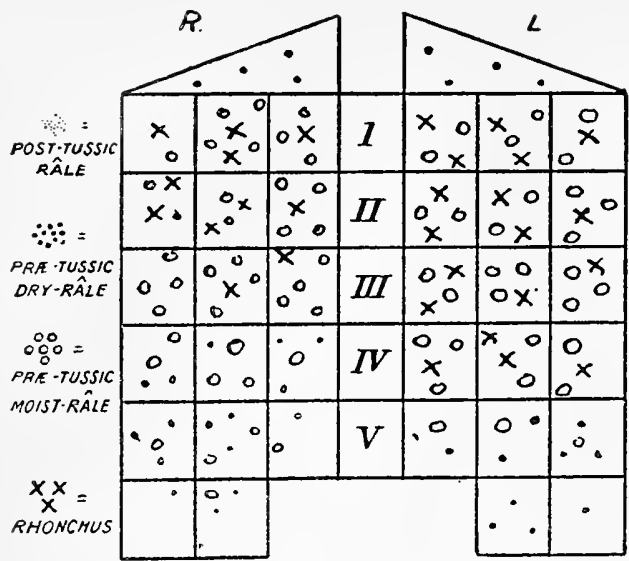

\begin{tabular}{|c|c|c|c|c|c|c|}
\hline$x_{x}^{0}$ & $\begin{array}{ll}x & 0 \\
0 & x\end{array}$ & $x_{0}$ & $I$ & $x_{0}^{0}$ & $x_{0}^{0}$ & $\therefore$ \\
\hline$x_{0}$ & $x=0$ & $\begin{array}{l}0^{x} \\
\times 0\end{array}$ & $\boldsymbol{I}$ & $0_{0}^{\circ}$ & $\times 0$ & $0 x$. \\
\hline$x_{0}^{0}$ & $\begin{array}{l}x_{0} \\
O_{X}\end{array}$ & $\begin{array}{l}0 \\
x_{0}\end{array}$ & $\boldsymbol{W}$ & $0^{x} 0$ & $\begin{array}{l}0 \\
\times 0\end{array}$ & $\dot{0}_{0}$ \\
\hline $\begin{array}{r}0 \\
\times 0 \\
\end{array}$ & $x^{0}$ & $x^{0}$ & $I V$ & $\dot{x}_{0}$ & $x_{0}^{0}$ & $\because 0$ \\
\hline$\because 0$ & $x^{\circ}$ & $x^{0}$ & $V$ & $0 x$ & $: 0$ & . \\
\hline \multirow[t]{2}{*}{$\because$} & $x^{0}$ & .0 & $V I$ & $\therefore \dot{0}$ & $\therefore$ & $\cdot$ \\
\hline & & - & $V W$ & & & . \\
\hline
\end{tabular}

CHART $15^{b}$.-Twelve hours after first inoculation. Sputum $=$ ro oz. per day.

Front of Chest

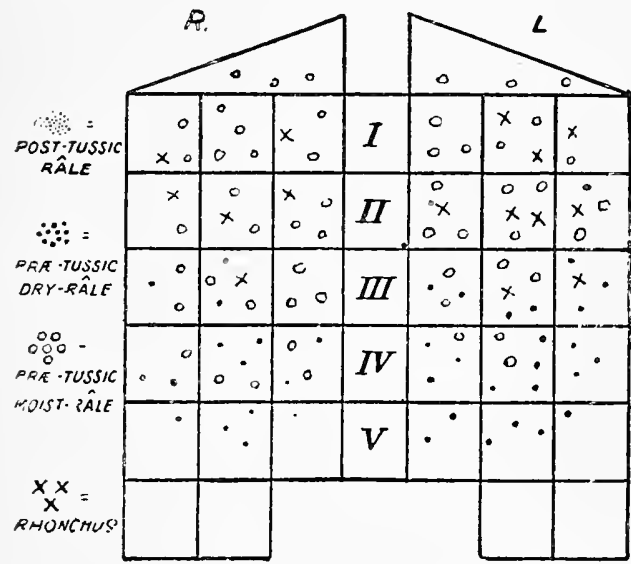

Back of Chest

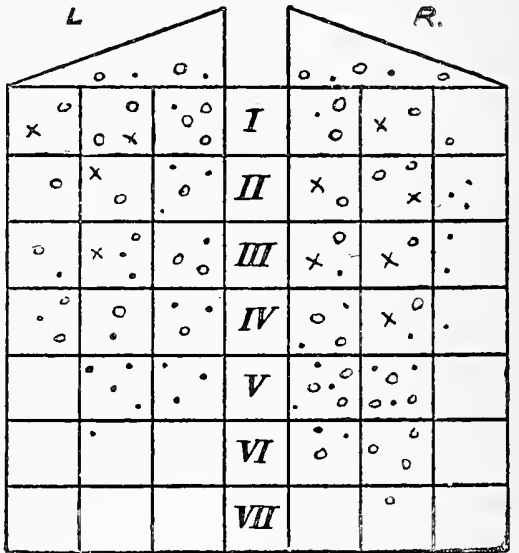

CHART I 5 . - Twenty-four hours after first inoculation. Sputum $=6$ oz. per day.

Front of Chest

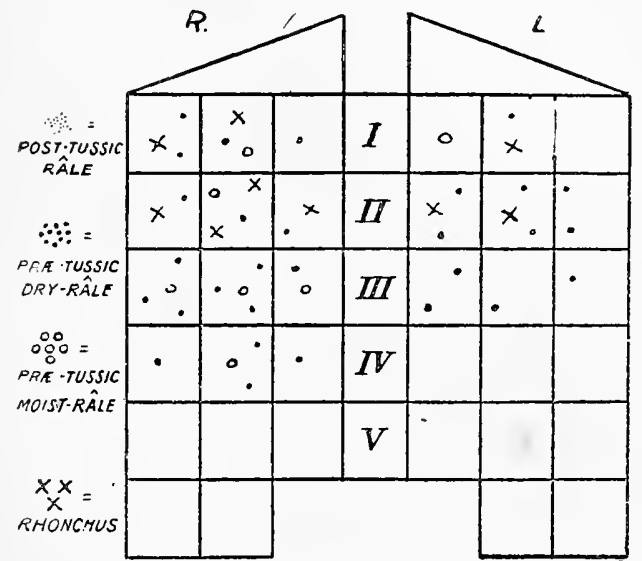

Back of Chest

\begin{tabular}{|c|c|c|c|c|c|}
\hline . & 0 & 0 & $I$ & & \\
\hline$x^{\prime}$ & $\times 0$ & 。 & $\pi$ & $x$ & $x$ \\
\hline . & $\because$ & . & III & $\because$ & $x$. \\
\hline & $\because$ & 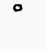 & $I V$ &.$^{x}$ & . \\
\hline & & & $V$ & & \\
\hline & & & $V T$ & & \\
\hline & & & $V I$ & & \\
\hline
\end{tabular}

$\mathrm{CHART}_{\mathrm{H}} 5^{2}$.-Forty-eight hours after first inoculation. Sputum $=2 \mathrm{oz}$. per day. 
Front of Chest

Back of Chest

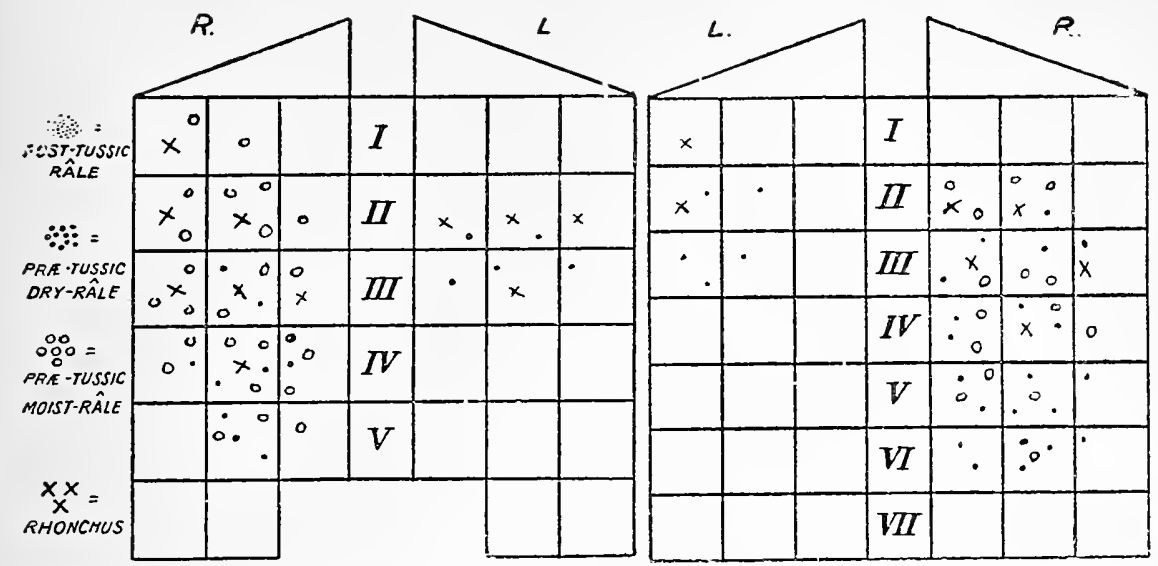

CharT I $5^{p}$.-Six days after first inoculation. Sputum $=3$ oz. per day. Second inoculation.

Front of Chest

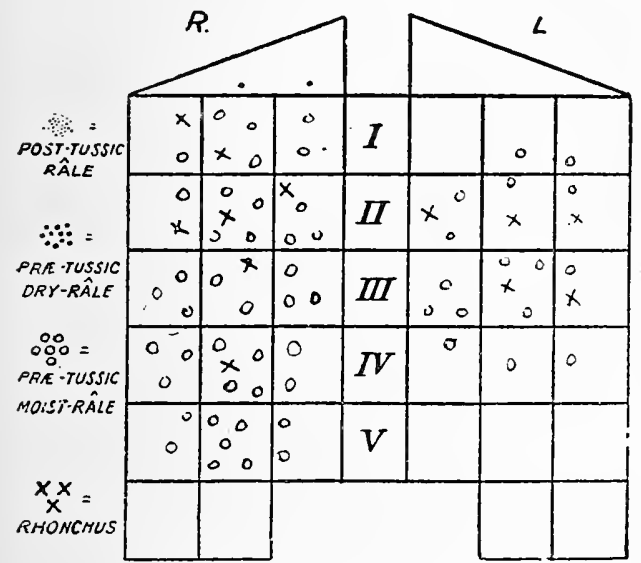

Back of Chest

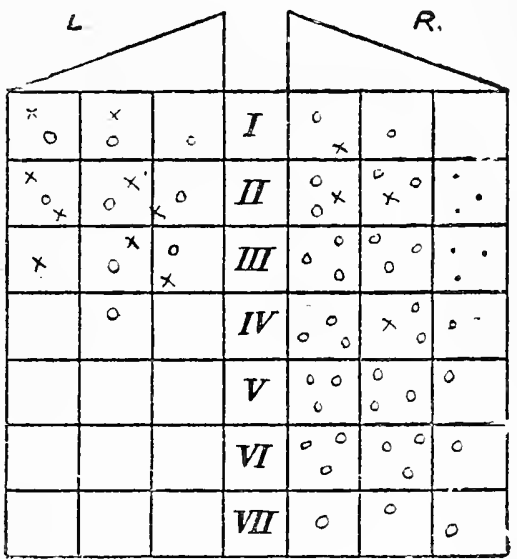

ChaRT 15 . - Twelve hours after second inoculation. Sputum $=+$ oz. per day.

Front of Chest

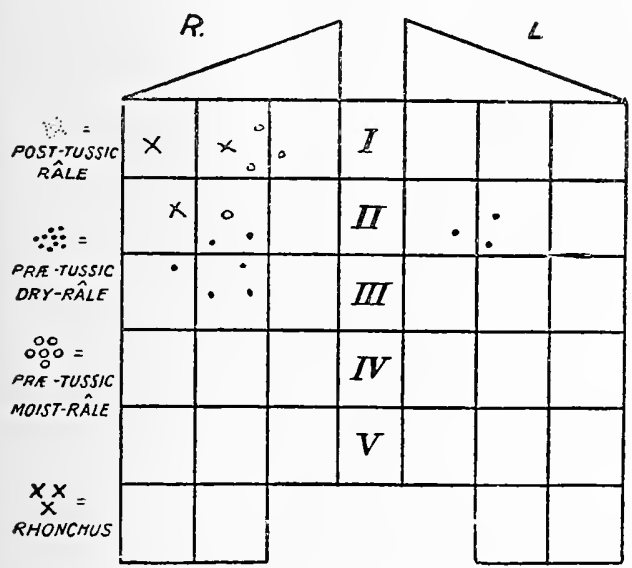

Back of Chest

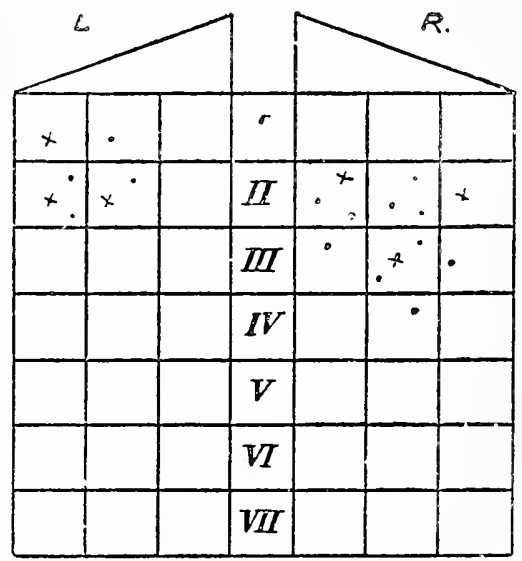

CHART I $5^{g}$.-Forty-eight hours after second inoculation. Sputum $=\frac{1}{2}$ oz. per day. 
patient generally and upon the physical signs in particular than do others. The interesting fact now comes out in the great majority of cases that the vaccine which will produce the greatest effect is of that organism which likewise causes in vitro the greatest acceleration upon the growth of the $B$. tuberculosis, and that this organism is not necessarily the one which predominates either in smears or in cultures of the sputum.

It is thus apparent that theoretically the way in which the fullest use is to be made of laboratory observations, and according to which the greatest benefit is likely to accrue to the patient, provided that the clinical condition warrants such delay, is to prepare immediately vaccines of all the allied organisms, to estimate the accelerating influence of the toxin of each, and then to begin treatment with the vaccine of that organism which produces the greatest accelerating effect. So much time has been occupied in the preliminary stages of these investigations that opportunity for the application of the lessons learnt has as yet been scanty.

In the instance, however, of Case 2, illustrated by figs. $40^{4}, 40^{\mathrm{b}}$, $40^{\circ}$, Plate IX, this procedure was adopted, with the striking results that are depicted in Charts $16^{a}$ to $16^{h}$.

The clinical history was as follows:

A male, aged 40 years, had had practically no illness till two years prior to my seeing him; enlarged glands then appeared on the right side of the neck; suppuration followed, then healing. Nine months later a severe cough came on, followed by pneumonia and pleurisy for which he refused to take to bed: three months afterwards tubercle bacilli were found in the sputum. Although his general condition had remained good his appetite had become very bad, and there had been a loss of weight of 8 to ro lbs. There had been no night sweats and the bowels were regular. There was a nightly rise of temperature to between $100^{\circ}-\mathrm{IOI}^{\circ} \mathrm{F}$.

On examining the patient the clinical condition briefly was as follows: In the mouth eleven stumps were seen and very advanced pyorrhœa alveolaris. To this cause the enlarged glands were attributed. These ran down the right side of the neck, each constituent being about the size of a hazel-nut.

The left side of the chest moved badly and was somewhat retracted, and there was marked sinking in over the left clavicle. There was amphoric resonance from the first to the fourth left ribs in front, and below the level of the nipple there was marked dulness.

Behind there was absence of resonance above the clavicle, and very marked dulness from just below the spine of the scapula to the level of the eighth rib. 
The right side appeared normal back and front, both to inspection and percussion.

On auscultation there were crackling rales to be heard all over the left chest, back and front, and some rhonchi; over the space in front extending from below the first rib to the level of the fourth rib cavernous râles were audible. Here also there was bronchophony and marked pectoriloquy. The only abnormality detected on the right side was a slightly prolonged expiratory murmur at the apex in front.

The diagnosis was made of advanced phthisis of the left upper lobe with great cavitation, consolidation of the whole left lower lobe with commencing softening, and some bronchitis. Infection of the right apex doubtful.

Front of Chest

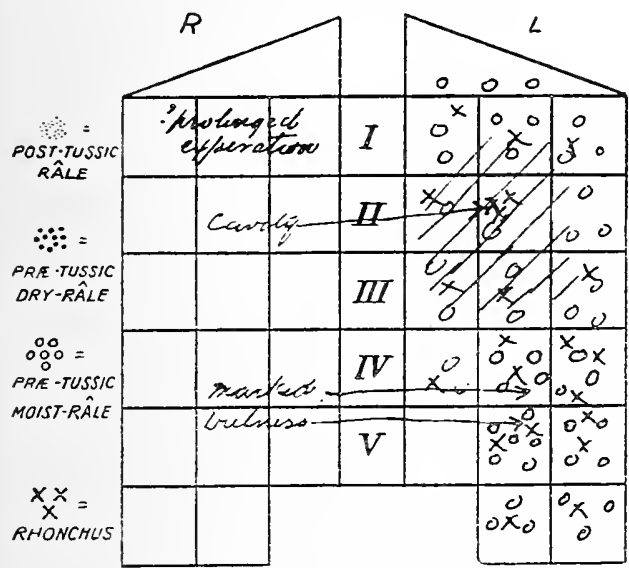

Back of Chest

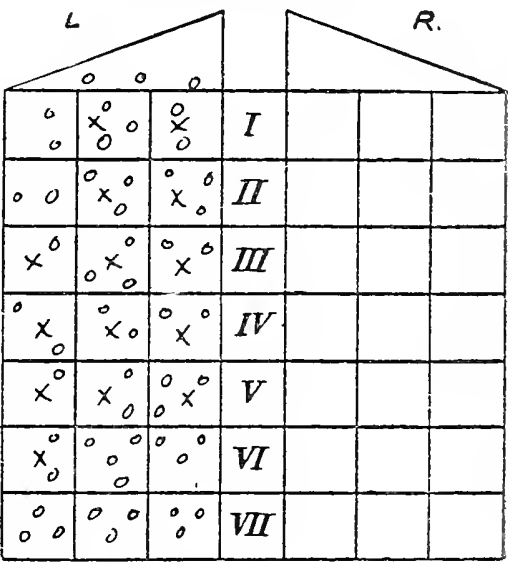

Chart 16 ${ }^{n}$.-Case 2, before inoculation with Vaccine I.

Specimens had previously been forwarded to me, and the tubercle bacillus, pneumococcus, Streptococcus longus isolated.

The patient was sent to bed, and on the second day the eleven stumps and few remaining sound teeth extracted; the sockets were sprayed repeatedly with hydrogen peroxide, and a chinosol mouthwash prescribed. Three days later the patient already declared that he felt better and that his appetite had improved.

As by the sixth day the evening temperature had remained steady for three consecutive days at $99^{\circ} 8^{\circ} \mathrm{F}$. and the morning one at $98^{\circ} 6^{\circ}$ $98 \cdot 8^{\circ} \mathrm{F}$. it was resolved to begin treatment with the streptococcal vaccine. Chart $I \sigma^{a}$ shows the then existing condition of the chest. A 50-million dose was given at 7 p.m., and subsequent observations made next morning at 9 a.m., at 7 p.m. at night, and at 7 p.m. on subsequent evenings. Charts $16^{b}, \mathrm{II}^{c}$, etc., illustrate the findings. 
Front of Chest

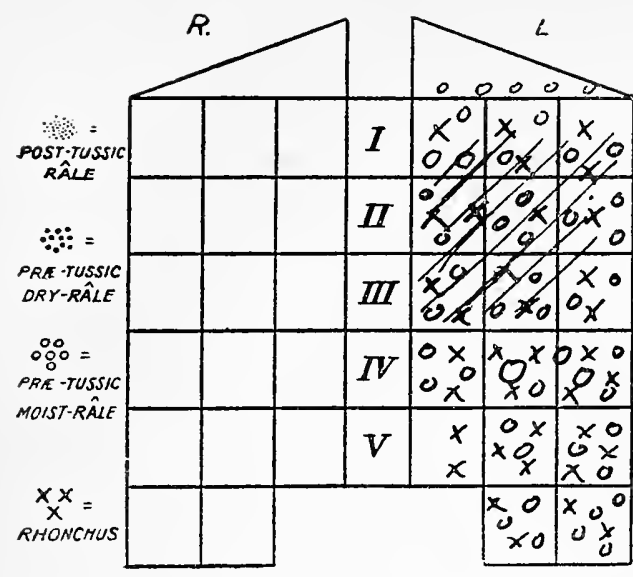

CHART $16^{b}$.-Case 2 , fourteen hours after inoculation.
Back of Chest

\begin{tabular}{|c|c|c|c|c|c|}
\hline $\begin{array}{l}0 \\
x^{2} \\
x\end{array}$ & $\begin{array}{l}x \\
o_{x}\end{array}$ & $\begin{array}{l}0 x \\
\times 0\end{array}$ & $I$ & & \\
\hline $\begin{array}{l}x \\
0 \\
0\end{array}$ & & $\begin{array}{ll}x & 0 \\
0 & x \\
0\end{array}$ & $\boldsymbol{I}$ & & \\
\hline $\begin{array}{r}x^{9} x \\
0 \% 0\end{array}$ & $\begin{array}{ll}0 & x \\
x & 0\end{array}$ & $\begin{array}{l}x_{0}^{0} \\
0^{\circ}{ }_{0}\end{array}$ & III & & \\
\hline $\begin{array}{l}0 \\
0 \times 0 \\
0\end{array}$ & 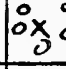 & $\left\{\begin{array}{l}x \\
x \quad \\
x \quad 0\end{array}\right.$ & $I V$ & & \\
\hline $\begin{array}{ll}x_{0}^{0} \\
0 \\
0\end{array}$ & $x_{0}^{0}$ & $\dot{0}_{0}^{0}$ & $V$ & & \\
\hline $\begin{array}{cc}0 & 0 \\
x & 0 \\
x\end{array}$ & $\begin{array}{l}\times 0 \\
00\end{array}$ & $0^{\circ}$ & $\boldsymbol{V}$ & & \\
\hline $\begin{array}{l}0 \\
0\end{array}$ & $\begin{array}{lll}x & 0 \\
0 & 0\end{array}$ & $\times 0.0$ & $V I I$ & & \\
\hline
\end{tabular}

Front of Chest

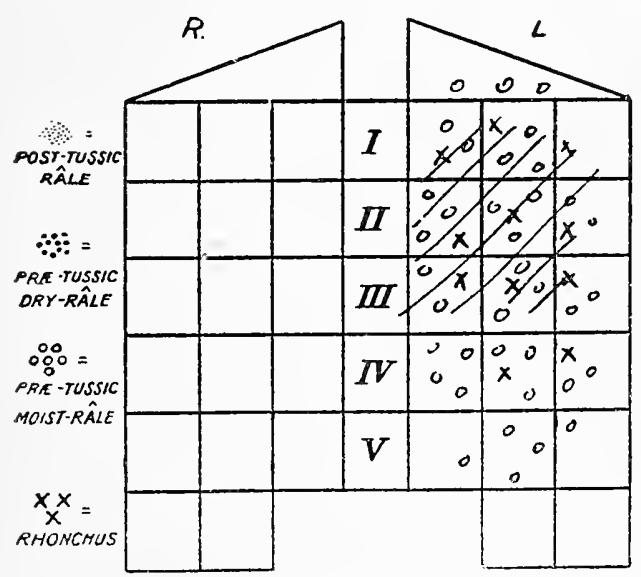

Chart $16^{c}$. - Case 2 , twenty-four hours after inoculation.
Back of Chest

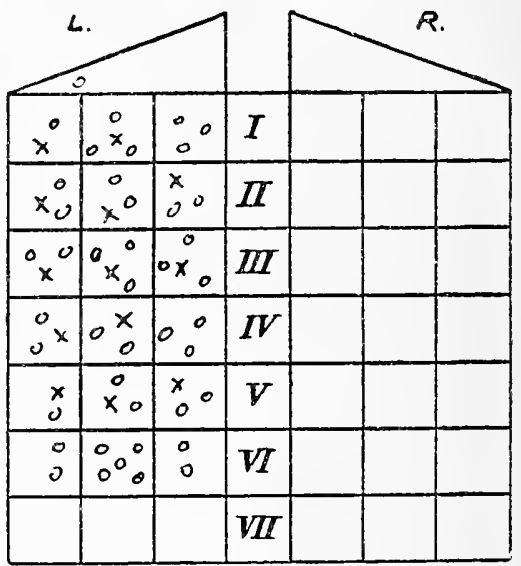

Front of $\mathrm{Chesi}$

Back of Chest

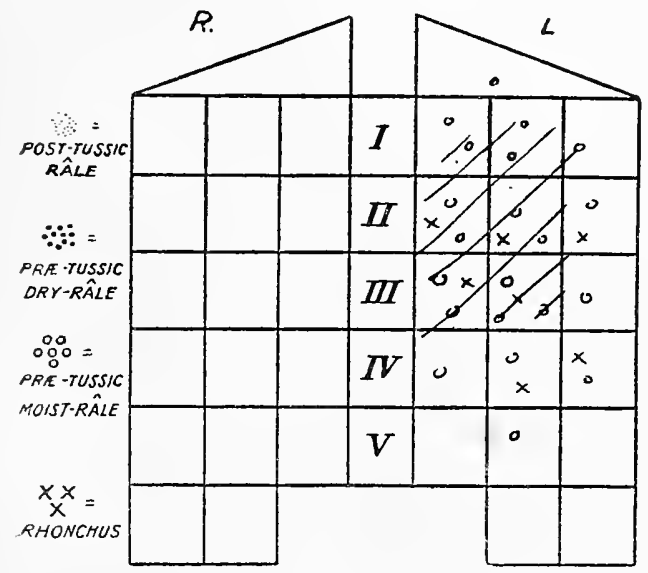

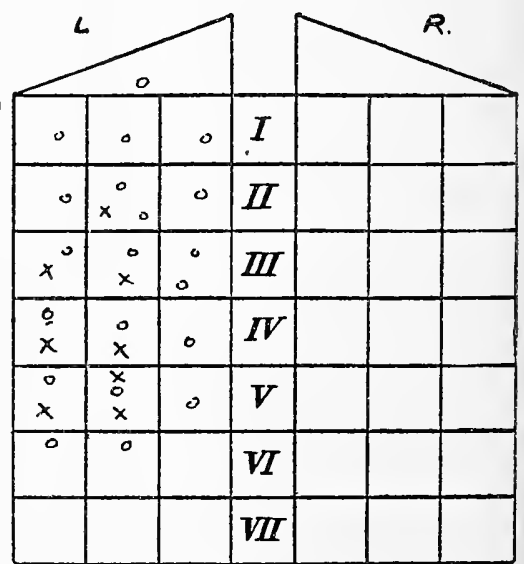

Chart i $6 a$. - Case 2, five days after first inoculation. 
Front of Chest

Back of chest

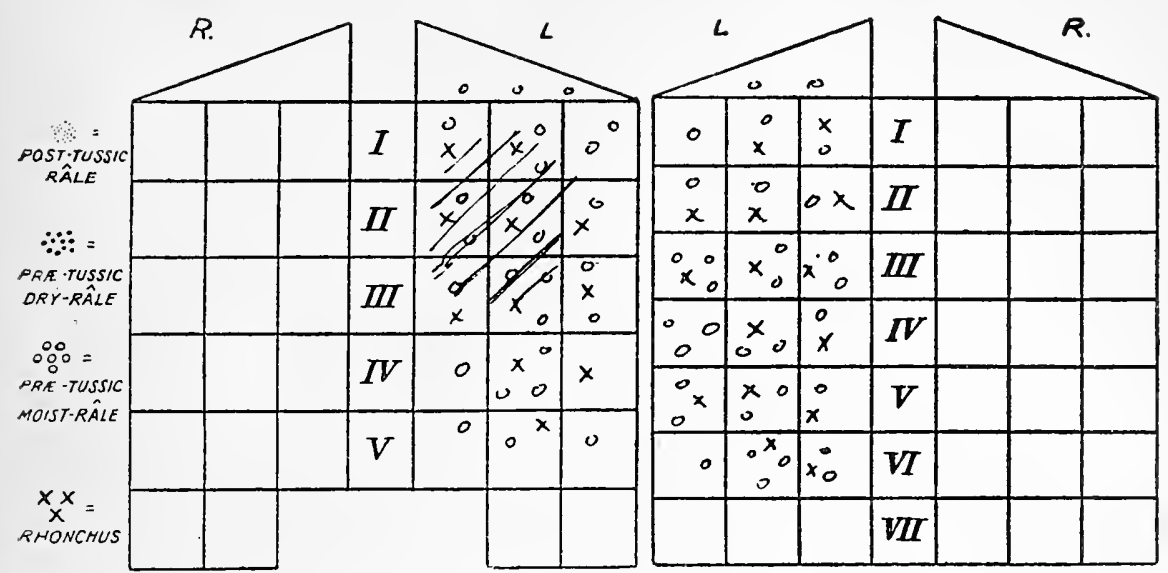

Chart i $6^{\circ}$--Case 2, six days after first inoculation; second inoculation Vaccine I.

Front of Chest

Back of Chest

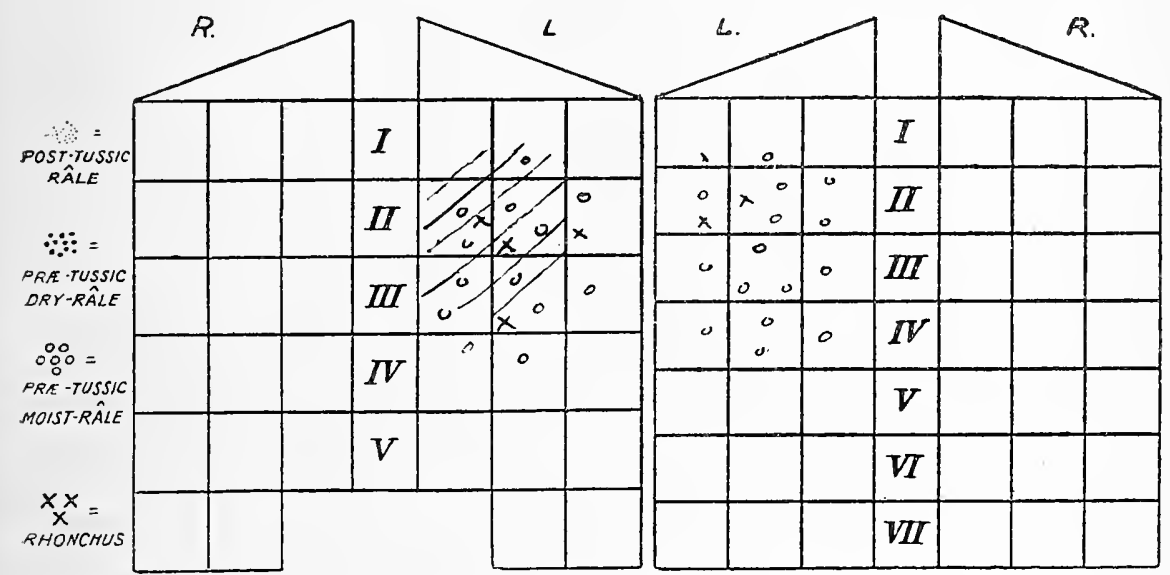

CHART $16 \%$-Case 2, eleven days after first inoculation; third inoculation Vaccine II.

Front of Chest

Back of Chest

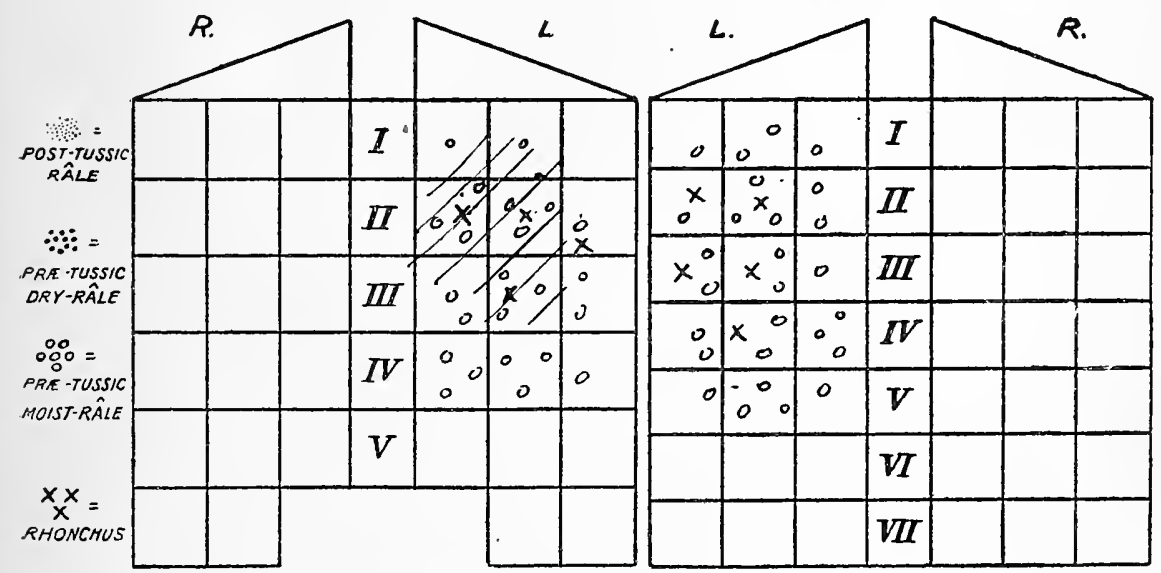

Chart 160.-Case 2, twelve hours after third inoculation. 
It will be observed that at the end of fourteen hours there was some exacerbation of the signs, and the temperature was raised $0.5^{\circ} \mathrm{F}$; by the end of twenty-four hours improvement had begun to set in, although the temperature was still raised $0.5^{\circ} \mathrm{F}$. Thereafter steady improvement continued till the evening of the sixth day, when there was a slight exacerbation. The 50-million dose of streptococcal vaccine was accordingly repeated, with the result that a similar sequence of effects was produced.

On the twelfth day of treatment, although there were no signs of relapse, I decided to give a 5o-million dose of the pneumococcal vaccine,

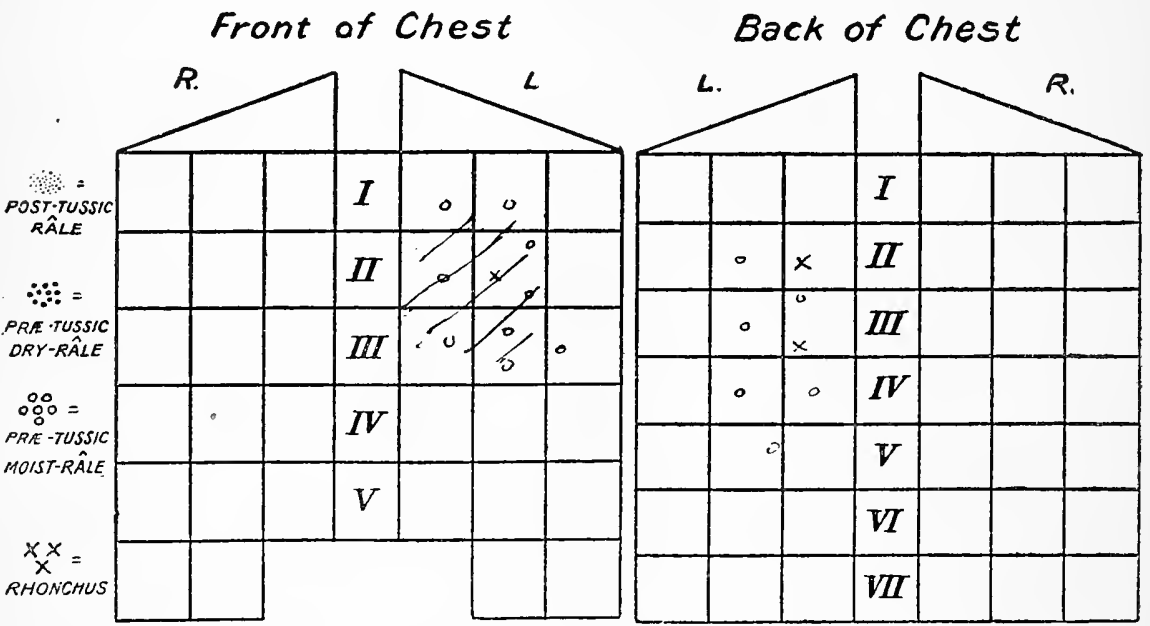

CHART $16^{\prime /}$.- Case 2 , fifteen days after first inoculation, left for home three days after third inoculation.

as the patient had to return four days later, and as he would pass out of my care thereafter I thought it well to ascertain the value of this vaccine, with a view to combining it, if found advisable, with the streptococcal one for employment by the doctor in the country.

A slight increase in the signs was noticed twelve hours later (Chart $\left.{ }^{1} 6^{g}\right)$, but this rapidly passed off, and when I examined the patient on the morning of his departure (i.e. the sixteenth day) I was more than gratified to find that the condition was that depicted in Chart $I 6^{h}$. The dulness over the lower lobe, back and front, had completely gone, the amphoric breathing, bronchophony and pectoriloquy were reduced, and only a very occasional small râle was to be heard anywhere. Even post-tussic râles could not be elicited elsewhere.

When seen four months later the patient was looking and feeling very well indeed; he had recovered all his lost weight, and an occasional fine râle at the end of inspiration was all that could be heard. 
The subsequent history of the patient was one of continued progress for a period of over two years, interrupted only by an attack of influenza, which soon yielded to treatment, and by a slight pyrexial attack set up by active exercise to escape the attentions of a very much excited bull. He then contracted a severe pneumococcal influenzal infection, neglected himself, and soon died.

The experience I had in this case of the effect producible by a suitable vaccine has been confirmed in other cases. Inasmuch, however, as the estimation of the accelerating effect of the toxins occupies about ten weeks of time, and this delay may prove anything but advantageous to the patient, two alternative procedures have suggested themselves:

(I) The prior estimation of the action of tuberculin upon the physical signs, which is carried out in precisely the same way, merely substituting for the vaccine such doses of tuberculin as are thought advisable ;

(2) The more or less empirical employment of vaccines of some or all of the organisms isolated from the sputum, choice being made of combinations which experience indicates as being likely to benefit the patient: this empirical choice is subsequently confirmed or modified by the laboratory experiments and by the progress of the patient.

The latter of these alternatives I consider the better, inasmuch as experience tells me that in the class of case which is likely to benefit from vaccine treatment, the effects produced by the vaccines are much more rapid and much more marked than would be those produced by any limited course of tuberculin therapy.

In illustration of the validity of this position, I will give a short description (with charts) of a case in which this procedure was adopted.

Mrs. A-, aged 48 years. History briefly as follows: About five vears before consulting me several hæmorrhages from the lung. Went to Midhurst for five months, where many tubercle bacilli were found, and the diagnosis made of tuberculous disease of both lobes of the right lung. Improved considerably, and told she was almost dry. IVent home, and almost immediately contracted a severe bronchitis, which threw her back. Remained at home under open-air treatment for about a year, then went to Rosa for six months. The high altitude did not seem to suit her, so returned to England in the summer, which she spent at Margate. Here she had a hæmorrhage from the bowel and some pyrexia, which persisted. Since then she had stayed in England, spending the last two winters at Falmouth. Despite the fact that she has had much mental worry, she was more than holding her own. Unfortunately last winter she was placed in a damp bed, con- 
tracted influenza, had several hæmorrhages, and lost ground considerably. This influenzal attack she has been unable to throw off. Her usual rectal temperatures have been $98^{\circ}$ in the morning, $99^{\circ}$ in the evening; sometimes $\mathrm{IOO}^{\circ}$ in the evening, and occasionally IOI - this when she contracts a cold, and to these she is very subject.

Her periods are regular, the evening temperature rising about $0.6^{\circ}$ for the preceding six or eight days and while they last, then falling to her normal. She has not lost weight, in fact the tendency to increase is such that she limits the amount of milk and cream in her dietary to counteract it. When I saw her she had been in bed for about two months and looked rather pale, but otherwise in robust health. Tubercle bacilli were very numerous in her sputum, allied invaders being the B. influcnia,

Front of Chest

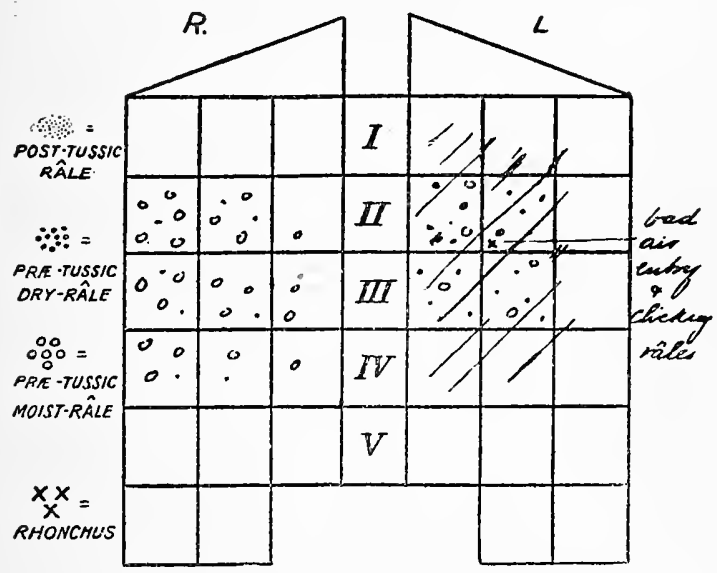

Back of Chest

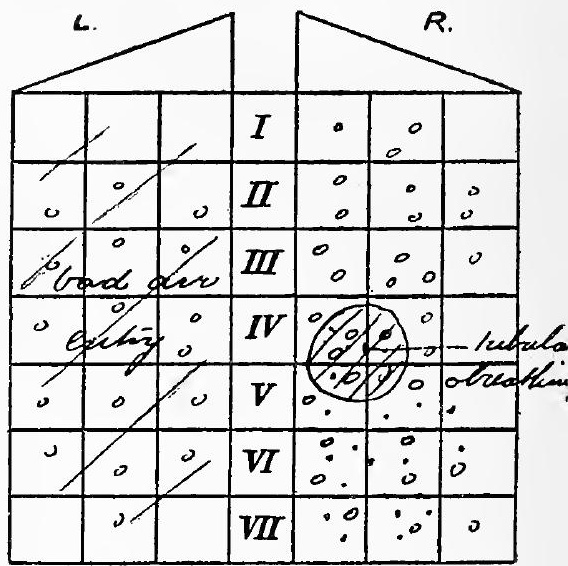

CHART $17^{a}$.-Mrs. A-, before inoculation with vaccine-B. Influenze 100 millions, M. catar. rhalis, 50 millions, pneumococcus and streptococcus each 25 millions.

M. catarrhalis, Streptococcus brevis and Pneumococcus. Her temperature rose in the evening to about $100^{\circ} \mathrm{F}$.

The main points revealed by clinical examination of the chest were as follows: it was well covered, there was no sinking in above the clavicles or elsewhere; the respiratory movements were poor, especially on the right side.

On percussion there was impaired resonance in front above the right clavicle and in the first space, and over the whole of the right chest behind. Elsewhere it was not quite satisfactory without being actually defective.

On auscultation the condition shown in Chart $I 7^{a}$ was found. In front on the left side was bad air-entry from the clavicle to the fifth rib; in the second and third space an occasional clicking râlc was heard at the end of expiration; in the fifth and sixth spaces external to the 
Front of Chest

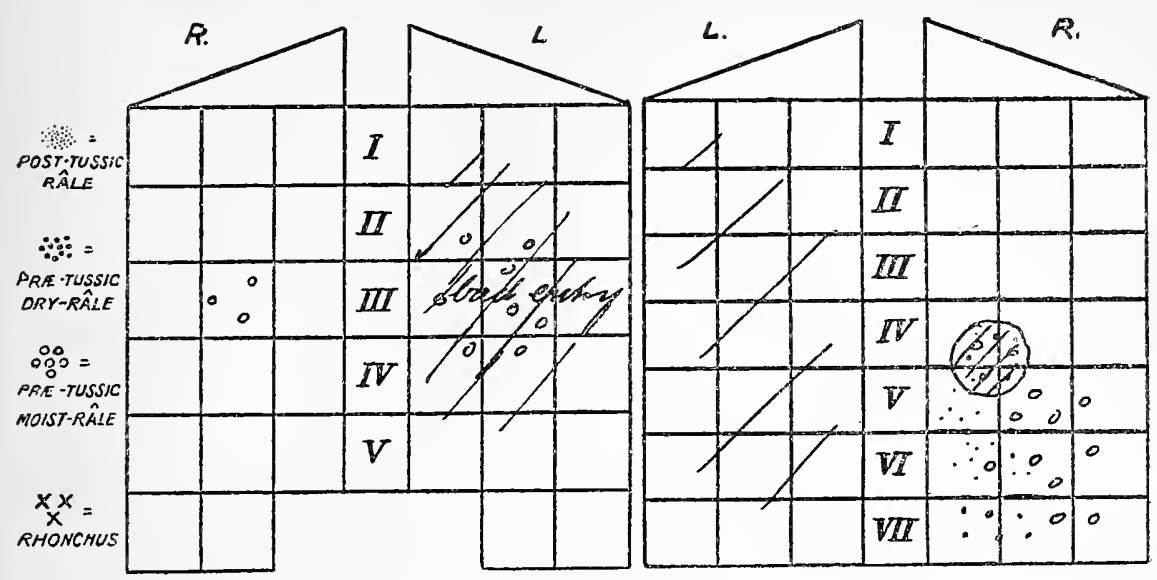

ChART I $7^{b}$ - Mrs. A-, eighteen hours after inoculation.

Back of Chest

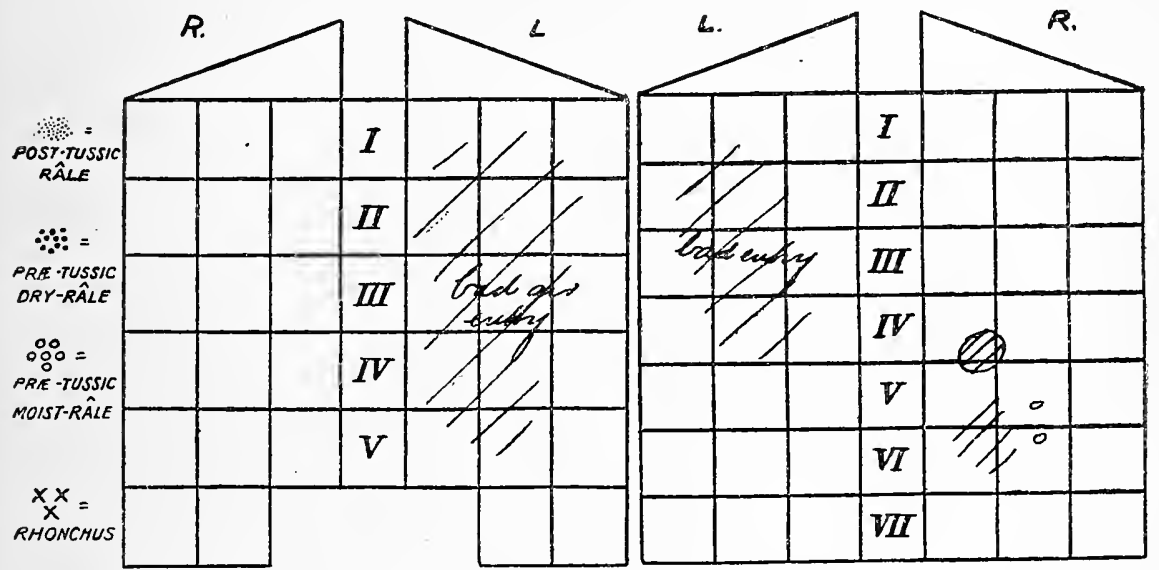

Chart I $7^{c}$.-Mrs. A-- five days after first inoculation; similar condition on twenty-sixth day.

Front of Chesi

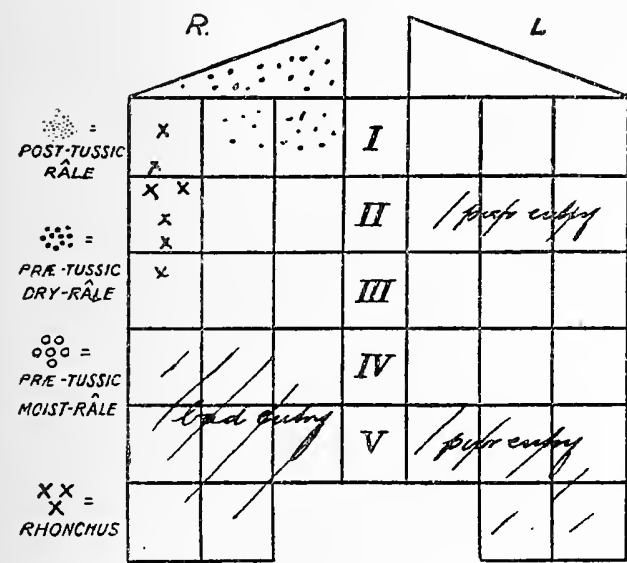

Back of Chest

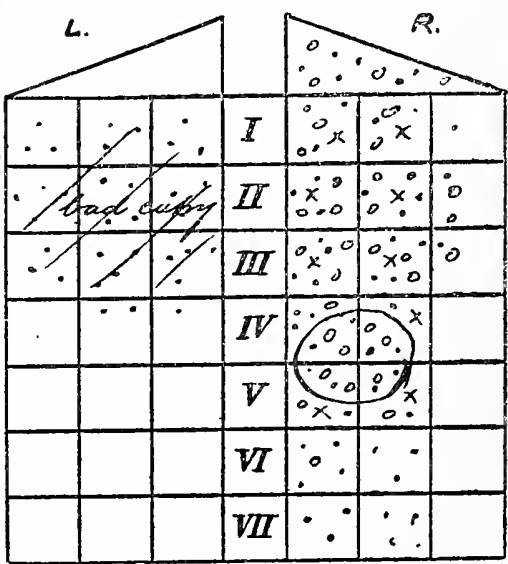

Chart I $7^{d}$.-Mrs. A - eighteen hours after fourth inoculation, which equalled one and a half times initial dose. Looking pale and "feeling rather out of sorts." 
Front of Chest

Back of Chest
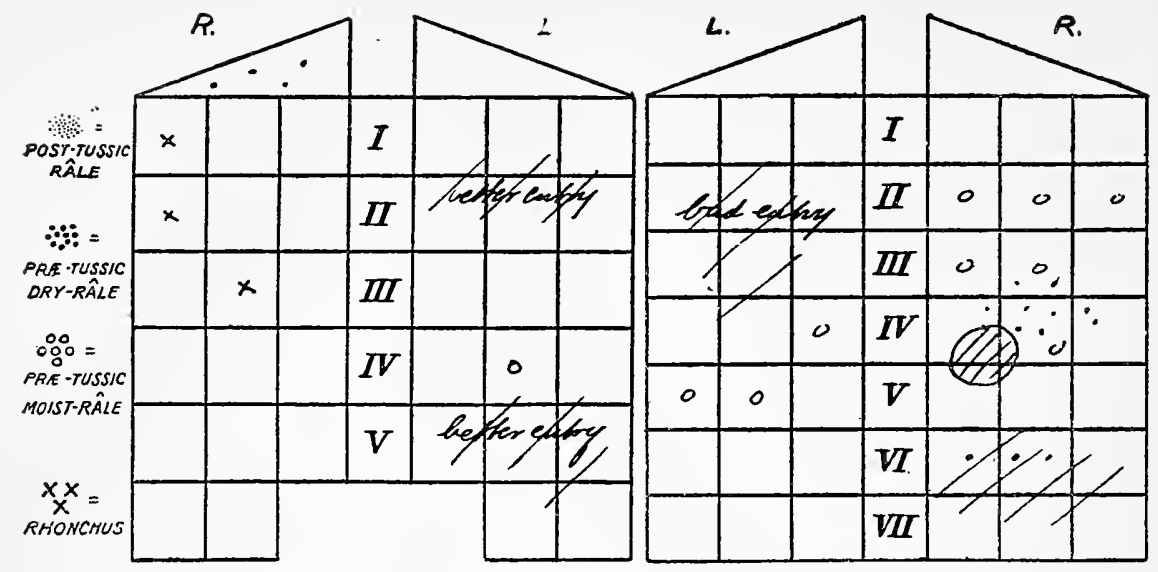

ChART I $7^{\circ}$.- Mrs. A-, forty-eight hours after fourth inoculation.

Front of Chest

Back of Chest

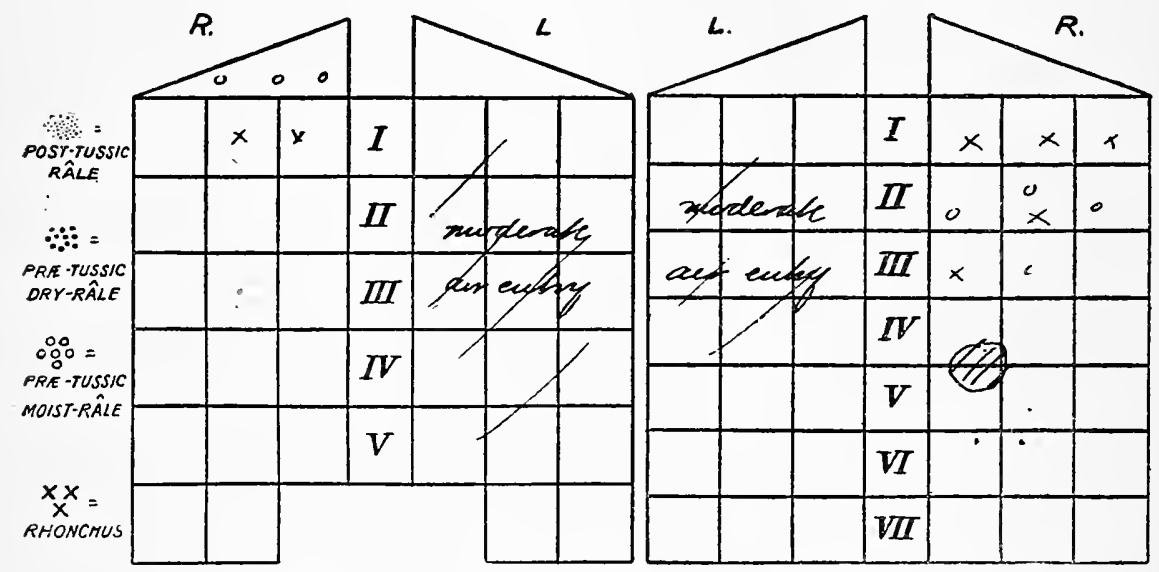

ChART i $f^{f}$.-Mrs. A-, before sixth inoculation (of double initial dosage).

Front of Chest

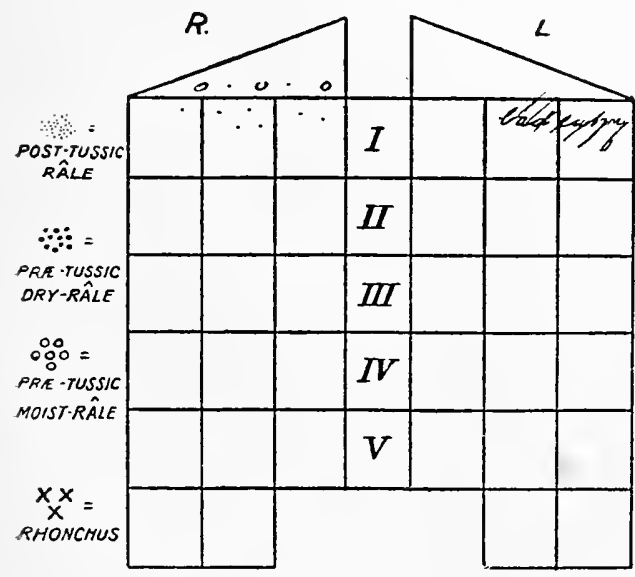

Back of Chest

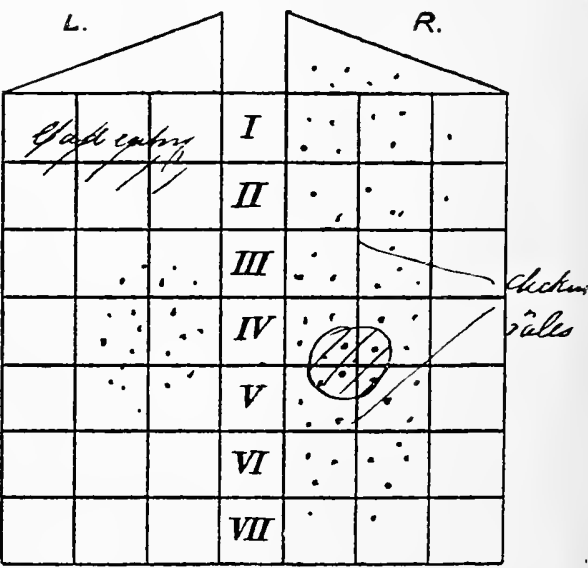

CHART I $7^{g}$.-Mrs, A-, twenty-two hours after ninth inoculation. 
nipple line there was "sticky" breathing. On the right side in the second, third and fourth spaces there were whistling rates, especially noticeable in the axillary line. Behind there was bad air-entry over the whole left chest, with an occasional moist rale from the second to the seventh space. Over the whole of the right side from below the level of the clavicles there were whistling rales, and in the fourth and fifth spaces near the middle line tubular breathing was to be heard over an area nearly three inches square. An autogenous vaccine was prepared, and an initial dose containing I oo million $B$. infuenza, 50 million $M$. catarrhalis and 25 million each pneumococcus and Streptococcus breit is was given.

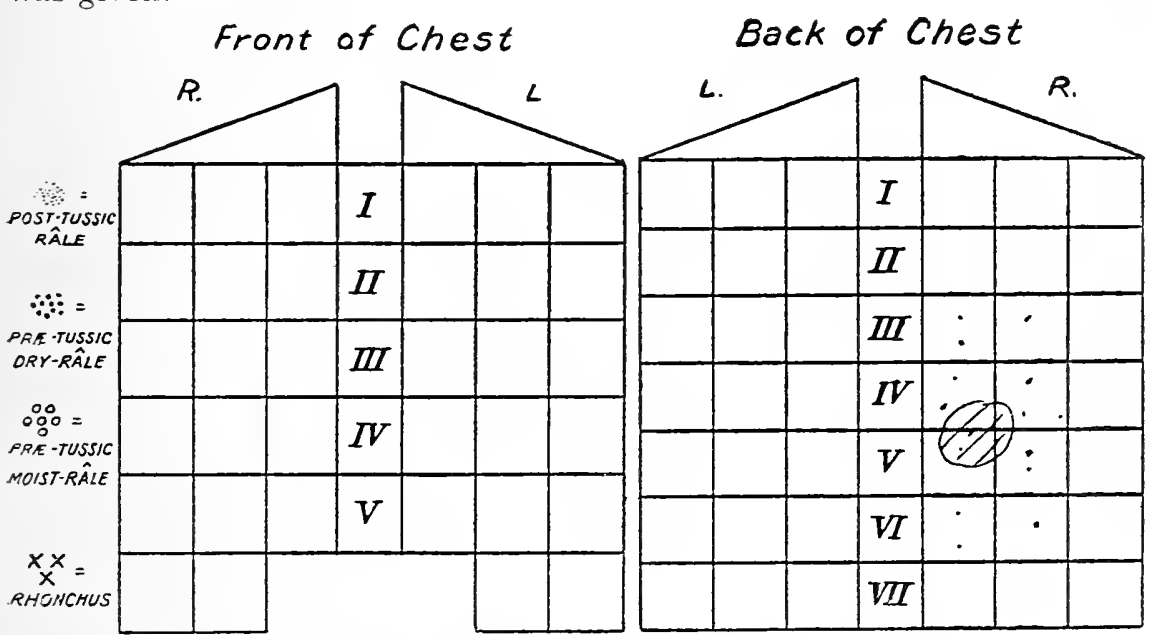

CHART I $\%$ - Mrs. A-, forty-eight hours after ninth inoculation.

Opportunities for making the regular and methodical examinations which I have already described as so advisable were lacking, but the progress of the case can be sufficiently' well followed in the various charts. Twenty-four hours after this inoculation the condition of the chest was as shown in Chart $\mathrm{I} 7^{b}$; there had been a marked clearing of the chest; the fact that this happened so rapidly, taken in conjunction with the fact that there was practically no general reaction, showed that the dose if anything erred rather on the small side; still, as the improvement continued and there was no retrogression five days after inoculation (see Chart $I T^{c}$ ), the dosage was maintained and repeated, as also on the twelfth and nineteenth days. It was reported to me on the twenty-sixth day that no moist sounds were to be heard in the chest, that the area of tubular breathing was smaller, but that the air-entry was still bad and that the state of the chest might fairly be considered to be that represented in Chart $I 7^{c}$. I therefore advised that the dosage for the 
fourth inoculation should be increased by one half and saw the patient eighteen hours later. The reaction was very marked (see Chart $I 7^{d}$ ); numerous râles were to be heard above the right clavicle and a few in the first right space in front. The whole right chest behind was filled with coarse râles and rhonchi, while on the left side behind fine râles were to be heard in the first, second and third spaces at the end of expiration. These signs, however, quickly subsided, the condition forty-eight hours after inoculation being that shown in Chart $I 7^{e}$.

This dosage was accordingly repeated eight days later and as there was only a quite moderate resultant reaction on the following days double the initial dose was given at the sixth administration. This produced no general reaction and no flaring up of the clinical signs comparable to that brought about by the fourth inoculation; on the contrary a slight but definite improvement resulted and persisted for the subsequent six days, when this dosage was repeated. Once again the increase of physical signs was very slight.

Subsequently the area of tubular breathing steadily diminished, the temperature failed to rise above $99^{\circ}$, the sputum became much reduced in quantity, and the patient was soon able to get about the house and maintain her steady improvement. It will be seen from Chart $I 7^{h}$ that practically the whole of the clinical signs in this case cleared up under the exhibition of bacterial vaccines alone, and when it is remembered that this is a case of quite long-standing phthisis it serves well to illustrate what I have already alluded to, viz., the impossibility of ever saying beforehand how much of the signs are due to the infection by the $B$. tuberculosis and how much to the mixed infection.

As soon as large doses of vaccine entirely fail to produce any immediate reaction or any further improvement in the patient I shall advise that treatment be begun with tuberculin, and be controlled in precisely a similar manner. A discussion of the tuberculin therapy of the case does not fall within the scope of this chapter, but attention may be drawn to the fact that suitable doses of tuberculin will certainly elicit responses in this chest and produce reactions therein, signs becoming obvious within twelve to twenty-four hours where none were existent at the time of inoculation.

The fact that all physical signs have disappeared in a chest is by no means proof of the disappearance of all infection. They have so departed from this chest, but vaccines can hardly have influenced the infection by the tubercle bacilli, and these are still being discharged in the sputum.

This, then, has been the method of choice which I have followed during the past two to three years in the treatment of all cases of pulmonary phthisis which have passed through my hands. I have 
been enabled to demonstrate the changes to numerous medical men well skilled in the use of the stethoscope and to two or three specialists in pulmonary tuberculosis; their observations of the changes produced have been described in such terms as "staggering" and "absolutely" incredible if not observed for oneself," and such indeed the changes are that are often brought about by the administration of suitable, skilfully. prepared vaccines in adequate dosages at suitable intervals. But let it not be thought that dramatic changes such as these can be secured in every case. Sometimes-and this even when the laboratory determinations show subsequently that there is no lack of accelerating action upon the growth of the tubercle bacillus by the allied toxins-progress is relatively slow: slight reactions are obtained, but the return wave does not carry the patient much past the starting-place. A little gain after each inoculation, howerer, means a considerable gain by the end of four to six months, and little by little the desired result is brought about. The important point to be remembered is that so long as a distinct reaction is produced in twelve to twenty-four hours there should be no increase of dosage; eren if the resultant improvement after six to eight days is hardly perceptible, that dosage which is effective in regard to the production of reaction must be persisted in till it shows a marked tendency to prove ineffective, when it may be doubled. The fact that four or five inoculations, each productive of an adequate reaction, do not appear to produce a satisfactory advance in the condition of the patient is eminently suggestive that the raccine is not a complete one, some organism not being included in it which is of considerable importance in the rtiology of the condition. A thorough examination of the sputum should elucidate this point. The practice of making systematic examinations of the sputum every fortnight is strongly to be advocated as tending to exclude such a cause of failure. It by no means follows in a case of multiple infection that a complex vaccine, however skilfully blended at the beginning, will prove efficient to the end of treatment. It is a phenomenon constantly encountered that in mixed infections one organism will be more easily eradicated than another, and that as one organism dies out another comes to the front: for instance, in mixed influenza-pneumococcal infections at the beginning of treatment there may be in a unit mass of sputum roo,00o B. infucnia and roo pneumococcus; at the end of a month's treatment these figures may be rooo B. influeña and Ioo pneumococcus. This does not indicate total failure to produce immunity against the pneumococcus. That the pneumococcus or its toxin produces a very marked acceleration in growth of the $B$. influenza I have often demonstrated. and the marked reduction in the numbers of the B. influen:e may well indicate a reduction in this effect referable to the pneumococcus, and so perhaps 
a reduction in the pathogenicity and vitality of the pneumococcus as well as an increase in antibodies to the $B$. influenza referable to the inoculations of $B$. influeña vaccine. None the less a good effect will usually ensue upon increased dosage of the pneumococcal vaccine, that of the B. influcnza being maintained.

Or, again, when the vaccine was first prepared the numbers of some one or more organisms may have so enormously preponderated over those of another variety that either the presence of the latter altogether escaped notice, or its isolation proved to be impossible. In such a case the exhibition for six to eight weeks of the vaccine first prepared, although a well-marked reaction follows each administration, may result in no apparent or well-marked improvement in the clinical condition of the patient. Examination of the sputum will almost surely show that the vaccine has not been without effect; the organisms of which the vaccine is composed may have almost disappeared, but the one which previously could not be isolated has come so much to the front that it has sufficed to maintain the clinical condition more or less in its original state; the exhibition of a fresh vaccine of this organism will almost certainly produce the desired result.

In view of these facts I personally prefer to keep my several vaccines separate in a.case of multiple infection, and blend them in the required dosages prior only to each inoculation.

Another disturbing factor which will be revealed by methodical examination of the sputum is the acquirement of a totally new infection. This is especially liable to occur during catarrhal epidemics, and may prove a source of great trouble. The old vaccine upon which perhaps the patient was doing so well may have to be discarded and a fresh one prepared to meet the emergency. When the catarrhal infection has been overcome the resumption or not of the old vaccine will be determined by a bacteriological examination. An occurrence such as this proves not only so disturbing to the patient, but often so prejudicial that no effort should be spared to obviate it. Two measures which I have found most effective are $(a)$ the prevention of access to the patient of all sufferers from acute nasal or bronchial catarrhs; $(b)$ the periodic immunisation of the patient against acute catarrhs. This is best done by the administration every six months of the 50, IOO, and 250 million doses of the combined vaccine for colds of the Wimpole Institute; the three doses being given in ascending series at intervals of seven to eight days (see also p. I83).

"But," it may be asked, "do all cases of mixed infection of phthisis, when treated with the appropriate vaccine or vaccines under careful control as to dosages and intervals by the clinical and bacteriological methods which have been described, respond favourably to treatment?" 
My answer to this is, omitting for the present those cases in which vaccine treatment appears definitely to be contra-indicated, that there would appear to be a small number of cases in which no apparent good results from such a course of treatment. I have myself met with only one such case. This I will first describe, and then attempt the explanation.

Miss B-C, aged 37 years, early in I 900 contracted pleurisy. In December, Igoo, T.B. discovered in sputum. Went to Mentone, where her voice became husky. On her return a few months later, Dr. Greville MacDonald found tuberculous laryngitis. Local treatment did not prove very successful. Went to Neyland Sanatorium from January, I902, to March, 1903, where her voice improved. The pulmonary condition, which was confined to the right upper lobe, remained almost stationary. Went to live at Clacton. By October, 1907, the voice had become so husky and breathing so difficult that Dr. Greville MacDonald performed tracheotomy. Early in Igos she returned to Neyland for six to seven months. In June, Igog, her voice began to improve, and by January, IgIo, had become fairly strong.

When I saw her she complained chiefly of a very bad cough, and of great difficulty in voiding the rather scanty but very purulent sputum; she was very thin, and her appetite was very bad. At the right apex in front there was cavernous breathing with bronchophony and pectoriloquy-only a few dry râles were audible. Over the right apex behind there was impaired resonance, and an occasional râle was to be heard.

As Dr. Greville MacDonald was not available, I sent her to Mr. G. S. Hett for examination of her larynx. He reported advanced disease of the left side, with œdema of the right side and of the inter-arytenoid cartilage.

Examinations of her sputum showed great numbers of tubercle bacilli and of Staphylococcus albus, no other organisms being detected.

This was obviously a case of very slowly progressive phthisis and tuberculous laryngitis.

Into a full account of the treatment, which was continued for two years, I do not propose to enter. Tuberculin T.R. and B.E. and a vaccine of Staphylococcus albus were employed. Opportunities for making the careful clinical observations which I have already described as so advisable were lacking, so that I was not in a position to determine how far and in what way the patient responded to the vaccine. I can only say that she appeared to make slow but steady progress; this, however, I quite convinced myself was referable, if to the treatment at all, solely to the tuberculin. Although the staphylococcus vaccine was persisted with and utilised in dosages varying from Ioo to 4000 million organisms, it never seemed to produce the least 
effect upon the staphylococcus infection ; if anything, the numbers of this organism showed a slight increase. The amount and character of the sputum showed no alteration, except in a reduction in the numbers of the tubercle bacilli : these had almost disappeared when the patient contracted a severe pneumococcal-influenzal infection-her first catarrhal infection during two years-and succumbed in less than three weeks.

As personally I had never seen such an utter failure on the part of a vaccine to influence in the lung or any other part of the body a bacterial infection I endeavoured to elucidate the cause, and for this purpose devised the laboratory experiments I have already described. The vaccine, I may mention, was several times re-made, to eliminate the possibility of errors in its manufacture. The experiments taught these interesting points :

(I) That the toxin of this particular Staphylococcus albus was absolutely without an accelerating influence upon the rate of growth of this particular tubercle bacillus - if anything, there was a very slight inhibitory action. I repeated this experiment three or four times, remaking the toxin and employing fresh batches of culture medium, always with the same result (cf. figs, $42^{\text {i }}$ and $42^{b}$, Plate X).

(2) That this Staphylococcus albus toxin was capable of markedly stimulating the growth of the strains of the B.tuberculosis derived from other cases of pulmonary phthisis.

(3) That the rate of growth of this particular tubercle bacillus could not be influenced by toxins whose powers of stimulation upon other strains of the tubercle bacillus had been repeatedly demonstrated (cf. figs. $42^{\mathrm{a}}, 42^{\mathrm{c}}, 42^{\mathrm{d}}$, Plate X).

The only conclusion was that there was some subtle property probably peculiar to the patient's tissues which had so modified the tubercle bacillus as to render it incapable of stimulation by bacterial toxins, and which rendered nugatory all attempts to produce an artificial immunity to the Staphylococcus albus.

In this connection I may remark that the observation has been made by numerous clinicians that cases of pulmonary phthisis complicated by a Staplyylococcus albus infection frequently run a very chronic course. I have even heard of cases still living in whom cavitation was observed and Staplyylococcus albus found over thirty years ago.

So far as I am aware this is no general rule, and it does not appear to hold for a Staphylococcus aureus infection. There is thus a rough correspondence with the nature of the cutaneous lesions produced by these respective organisms.

In any future cases which I may be called upon to treat in which the Staphylococcus albus is the sole accessory microbe my procedure 
will probably be as follows: to begin treatment immediately with the staphylococcus vaccine, but if no good appears to be resulting by the time that the laboratory observations are completed, and these reveal a lack of accelerating powver on the part of the staphylococcal toxin, I shall discontinue the vaccine and rely upon tuberculin alone.

It may here be mentioned that I do not consider the use of combinations of vaccine and tuberculin to be advisable; vaccine and tuberculin each are capable of producing their definite effect upon the physical signs, an effect which can be accurately determined. If a combination be employed it is quite impossible to tell what precise effect each is producing, and all guidance as to dosages and intervals is lost. In cases where mixed infection is present the better procedure would appear to begin with suitable doses of the vaccine of that particular accessory microbe which experience indicates as likely to be a predominant factor in the production of the physical signs, or with a suitable combination of vaccines, and continue with this treatment according to the lines which I have indicated until laboratory investigations have determined the accelerating effect of each toxin. If no accelerating action is observed and the patient is not improving to discontinue vaccines and employ tuberculin.

If improvement is resulting, and laboratory observations enable the allied organisms to be sorted out into those with an accelerating influence and those without, to continue with the vaccines of the organisms which fall into the former category. The appropriate time to resort to tuberculin in these cases would appear to be when the vaccines cease to produce further marked amelioration; if it be done sooner difficulties are sure to arise, for when the patient begins to retrogress a little after each inoculation-signal for a fresh inoculation-a difficulty arises in deciding whether the fresh stimulus that is required is that to the formation of antibodies to the tubercle bacillus or to the allied organisms. Experience, however, is as yet very limited, and dogmatic expressions of opinion are much to be deprecated. As I have said before, every case of phthisis is a law unto itself, and what may prove the right course for one patient may be quite the wrong one for another.

The class of case in which vaccine treatment is likely to prove of little avail is unfortunately that for which its services are most required-I mean the cases of widely disseminated broncho-pneumonic phthisis. Mixed infection is rarely absent in these cases, but the number of accessory microbes present is as a rule extremely small. Pneumococcus and streptococcus are those most commonly found.

In the lobar form of acute pneumonic phthisis vaccines may or may not prove of service. In cases which begin as this variety vaccines are likely to prove an invaluable standby, but when this form 
supervenes upon a chronic attack vaccine treatment can hardly be anticipated to delay the end. The pneumococcus and Bacillus influenza are the accessory microbes most likely to initiate this change of type.

Summary of my Observations and Conclusions upon the Influence of Mixed Infections in Phthisis and upon their Cure by means of Vaccines.

(I) The allied micro-organisms found in phthisis may be either the truly primary infection, in which case they have initiated the tuberculous process by a prior manuring of the soil, or they may be secondary, in which case they exert not only an action upon the tissues. peculiar to themselves, but also, at times, an accelerating effect upon the rate of multiplication of the tubercle bacilli.

(2) The question whether vaccine treatment will prove of advantage to the patient or without effect may be indicated by the result of determinations as to whether the toxins of the accessory invaders produce any acceleration in the rate of growth in vitro of the autogenous tubercle bacilli.

(3) The treatment of cases should be begun with an autogenous vaccine, or mixture of vaccines, the precise influence of each dose being estinated by.measurements of the sputum, temperature and pulse-rate, and by the effect produced upon the clinical signs in the chest. That dosage is an effective one which within twelve hours produces a slight increase in the amount of sputum, slight rise of temperature and pulse-rate, and definite increase of physical signs. If these have passed off, or are beginning to pass off, in twenty-four hours the dosage is an appropriate one, but if no backward swing be evidenced within fortyeight hours excess of dosage is thereby indictaed.

The signal for repetition of the inoculation is a beginning recrudescence in the signs and symptoms; the indication for increased dosage a failure to react or an inadequate reaction on the part of the patient. Periodical bacteriological examinations of the sputum are highly necessary to eliminate fresh infections or an increased activity on the part of one of the accessory microbes, due possibly to the removal of the inhibitory action which may have been possessed by a variety which has been eliminated.

(4) Tuberculin and vaccine should not be given in combination, as the effect of one may mask the effect of the other, and confusion is sure to arise in estimating the influence of a given dosage, and the necessity for re-inoculation. Tuberculin should be withheld until marked improvement has been made under the exhibition of the vaccine and the patient is remaining more or less stationary, or until the conclusion has been reached that vaccine treatment is not likely to produce a good result. 
The tubercle bacillus and various allied invaders may each initiate changes in the lung tissues which give rise to symptoms practically indistinguishable. In chronic phthisis with mixed infection the clinical signs of activity may be almost entirely due to the secondary invaders. In such cases the proper exhibition of the suitable vaccine or mixture of vaccines may produce an amelioration of the condition which is very striking.

While it is perhaps the general rule that the allied invaders play the principal part in the production of the signs and symptoms, it is unwise to assume that this is always so. Careful adherence to the procedure which I have described will soon give indication that a change of treatment from vaccine to tuberculin is advisable.

(5) Experience is yet too limited to justify any dogmatic expression of opinion, but it would appear that in vaccine treatment we have a most valuable adjunct to other methods of treatment of cases of pulmonary tuberculosis complicated by accessory microbes. Pyrexia and frequency of pulse may be reduced, the appetite and weight increased, exacerbations and complications diminished, and great reduction in the physical signs brought about, so that large areas of lung-tissue which appeared to be deeply invaded by the $B$. tuberculosis may finally give no stethoscopic evidence of involvement; the way having thus been paved for the employment of tuberculin, if this be indicated or found to be necessary, a more rapid and more complete cure should then be obtained in a considerable proportion of cases.

It will perhaps not be out of place to make some short excerpts from well-known treatises on tuberculosis wherein the authors give their opinions of the scope and value of vaccine treatment in the mixed infections of pulmonary tuberculosis.

In Diseases of the Lungs, edit. 5, p. 627, Powell and Hartley say:

"We have given reasons for believing that in certain rapidly progressive cases of phthisis the fever and the spread of the disease are due in part to the agency of organisms other than the tubercle bacillus, which at least prepare the way for the activity of the latter, and the question arises whether by isolating such organisms from the sputum and producing appropriate vaccines their activity may not be cut short, to the relief of the patient and the improvement of his symptoms. In the few cases known to us which have been treated on these lines, the results have been, as a rule, disappointing, but in the following case (described) some improvement was apparently effected."

They conclude, "The treatment is still in its infancy, it is based on scientific lines, and it is one which demands a careful trial."

In Tuberculosis, edited by Klebs (rgo9), p. 598, Webb summarises 
as follows the results in fifty cases inoculated by him with homologous vaccines prepared from the mixed organisms:

"(I) In no case has a patient been harmed;

"(2) Many patients have had exacerbations more rarely;

" (3) Expectoration in nearly all cases has been lessened ; nocturnal coughs have frequently been eliminated;

"(4) In some cases a chronic catarrhal hoarseness has entirely disappeared ;

"(5) Concomitant pus affections have cleared away, such as suppuration of ears, staphyloccoccic acne and sycosis ;

" (6) When these vaccines have been combined with small doses of Koch's new tuberculin, spreading infiltrations have been averted and cleared up ;

"(7) In a case which displayed tubercle bacillus, streptococcus, pneumococcus, staphylococcus and $M$. catarrhalis, the latter was entirely eliminated by appropriate vaccine, and the amount of sputum was reduced from $4 \mathrm{Oz}$. to less than I oz. daily;

"(8) Evacuations of 4 to $6 \mathrm{oz}$. of sputum daily have in several cases been reduced to less than $\mathrm{I} O z$.

"The impression has been gained that the bronchorrhœa type of cases has, perhaps, received less benefit connected with the reduction of sputum than the cavernous type.

"In conclusion the author would put forth the earnest plea that these potent remedies be used early, while the machinery of the immunisation is yet unworn, and that they be added to the tuberculin treatment. Then the ranks of the advanced tuberculous will be lessened."

Pottenger (Pulmonary Tuberculosis, I908, pp. 257 and 26I) says:

"In the treatment of advanced cases of pulmonary tuberculosis we are often able (i.e. by general methods) to arrive at a result which is very satisfactory compared with the condition at the beginning of treatment. We can often secure a healing of all but a small focus, which keeps on secreting month after month. In such cases it seems probable that if we could but combine with tuberculin the appropriate vaccine made from the culture of the associated microbes we might heal these lesions completely. I believe we are safe in assuming that mixed infection is a factor in all cases of tuberculosis which have passed beyond the early stage of the disease and arrived at the open stage. I do not mean by this that an open surface in the airpassages is necessary for this association of other bacteria, for they should be as able to gain entrance to the tissues without an ulcerated surface as easily as tubercle bacilli, but I mention this open stage as showing a disease which is somewhat advanced. I do not doubt but. 
that the true remedy for mixed infection will be obtained in a raccine made from the cultures taken from the strain of the micro-organism found in each individual patient. The results which we had so far in our endeavours to treat in this manner are very encouraging."

Bonney (Pulmonary Tuberculosis, I9Io, pp. 9II-9IS) gives a very good account of the results he obtained in twenty-eight cases:

"In group $j$ are embraced patients exhibiting the persisting fever of mixed infection who were subjected to injections of homologous vaccines without tuberculin theraps. There are twenty-eight patients in this class displaying active tuberculous processes with profound constitutional disturbance. The vaccines were not administered to any patient in this group until the futility of hygienic measures without specific medication had been demonstrated beyond question. In view of the fact that the mixed infection is often the determining factor in turning the scales irretrievably against recovery, it was deemed justifiable to resort to the use of bacterial vaccines for patients apparently doomed to a fatal termination. An early control of the septicæmia offered the only rational basis for hope regarding nearly all invalids included in this group. . . . All individual vaccines were prepared from cultures grown from the secretions of the patient. In nearly. all cases the disease had been of long standing, the duration having been less than one rear in but three instances. While a few patients had been under observation from time to time during a period of sereral years, the acute septic manifestations had been of comparatively brief duration. . . . It is important to note that the twenty-eight cases are divided into two general subdivisions, the first twenty-two comprising one class, and the last six another. Among the patients in the former the physical condition was extremely urgent in all cases, the prognosis very unfarourable in five, doubtful in two and utterly hopeless in fifteen.

"In these cases the vaccine medication was employed in a last effort to reduce temperature and possibly aid thereby in saring or prolonging life.

"In the second sub-group very satisfactory improvement had been secured in each instance. The nutrition was excellent, with complete absence of fever, almost entire arrest of the activity of the tuiberculous process and a uniformly good prognosis. In these cases, however. the cough and expectoration represented important disturbing features. . . . In noting the results of vaccine therapy, it is necessary to discriminate between the apparently hopeless cases of one class and those without temperature elevation or other grave features in the second group. Of the twenty-two cases in the former, all of whom by virtue of every consideration ordinarily influencing prognosis were 
destined to an early fatal termination, eight are recorded as having achieved improvement to such an extent as to justify an unqualifiedly favourable prognosis, with almost complete arrest of the tuberculous process. Thus in more than one third of the cases the entire clinical picture was unexpectedly transformed, following vaccine medication. In six patients there was observed at first a material improvement, but this was followed by a subsequent retrogression, death taking place in each instance, although in one case as a result of a sudden pulmonary hæmorrhage. In seven instances no appreciable results were observed following the vaccine medication and the patients finally succumbed to the disease.

"In the second class, comprising six cases exhibiting a favourable prognosis, the vaccine was administered solely in the hope of controlling distressing cough and lessening expectoration. In one case the results were highly gratifying, but in the remaining five not the slightest influence could be properly attributed to the vaccine. These results suggest a much more limited field of usefulness for the homologous vaccines in afebrile cases than in those with acute septic manifestations. While generalising statements in connection with clinical observations of this character are hardly appropriate, the evidence appears conclusive that some benefit may be expected to attend the employment of such an agent in a fair proportion of cases otherwise adjudged incapable of improvement."

Reference to Bonney's table is necessary in order to appreciate the striking nature of the results achieved in some of the cases.

The prevention of mixed infection.-That prevention is better than cure is the soundest of axioms, and obviously, if it is possible to prevent the occurrence of any mixed infection complicating a pulmonary tuberculosis, this is a much better procedure than eliminating it when it has occurred. To this end it is necessary to consider the way in which infection by other bacteria than the tubercle bacillus arises in the lung. Bacteria'may be carried to the lung in any of three ways, (I) via the respiratory tract, i.e. either carried in the air current or by direct extension along the passages; (2) via the blood-stream; (3) by direct extension or viâ the lymphatics from some other internal focus, such as the abdominal peritoneum.

Instances of bacteria which reach the lungs in the first way are certainly the streptococcus, $M$. catarrhalis, M. tetragemus and paratetragenus, $B$. septus, diphtheroid bacilli, staphylococcus, and probably the $B$. proteus and B. pyocyaneus; and sometimes, if not usually, the pneumococcus and $B$. influenza.

Those which may reach the lung viâ the blood-stream are the pneumococcus and B. influenza, while one which perhaps usually reaches 
them by direct extension via the diaphragmatic lymphatics is the B. coli.

The origin of these various bacteria and the mode of their extension may be considered with advantage; and in this connection it at once suggests itself that all these bacteria may be divided into three categories: ( $\mathrm{I}$ ) those purely catarrhal in function; (2) those purely pyogenic; (3) those which are intermediate, sometimes producing catarrhal symptoms, sometimes giving rise to pus-formation.

Catarrhal organisms.

M. catarrhalis.

$M$. paratetragenus.

B. septus.

B. influenze.
Pyogenic organisms.

Staphylococcus.

B. protens.

B. pyocyaneus.

B. coli.
3

Intermediate.

Streptococcus.

Pneumococcus.

It accordingly follows that the focus from which the mixed infection has arisen must have been either a catarrhal or a suppurative one. If we except the $B$. coli, whose ingress to the lung is probably determined by the occurrence of a diaphragmatic pleurisy or of a peritonitis which perhaps has caused very little symptoms, we are at once struck by the thought that all the organisms which complicate cases of pulmonary tuberculosis are precisely those which cause common pathological conditions about the mouth, nose, naso-pharynx, upper and lower airpassages. The bacteria and the conditions they give rise to may be tabulated as follows:

Organism.

Streptococcus.

M. catarrhalis.

Pneumococcus.

$M$. paratetragenus. Staphylococcus.

\section{B. infuenze}

B. of Friedlander.
Conditions in which they may be concerned.

Post-nasal catarrh, pyorrhœa alveolaris, follicular tonsillitis, bronchitis, asthma, suppurative conditions in the mouth generally.

Nasal and post-nasal catarrh, pyorrhœea alveolaris, follicular tonsillitis, laryngitis, and tracheitis, bronchitis, asthma.

Nasal and post-nasal catarrh, pyorrhcea alveolaris, follicular tonsillitis, laryngitis, tracheitis, and bronchitis, asthma, pneumonia, infections of the accessory sinuses.

Nasal catarrh, laryngitis, tracheitis and bronchitis.

Pyorrhcea alveolaris, post-nasal catarrh, tonsillitis and quinsy, suppurative conditions in the mouth generally.

Nasal catarrh and infections of the accessory sinuses, tracheitis, bronchitis and pneumonia.

Nasal and post-nasal catarrh, bronchitis, pneumonia. 
Organism.

\section{B. proteus.}

B. pyocyaneus.

B. septus.
Conditions in which they may be concerned.

Suppurative conditions of the accessory sinuses and of the mouth generally.

Suppurative conditions of the accessory sinuses and of the mouth generally.

Nasal and post-nasal catarrh and pharyngitis.

If we except those rare cases of pneumonia and bronchitis which do not originate in a catarrh of the upper passages, it at once becomes evident that the conditions of prime importance are catarrhs of the upper passages, pyorrhœa alveolaris (and carious teeth), tonsillitis and suppurative conditions of the mouth generally; and it therefore follows that those measures which are likely to preclude the occurrence of mixed infection in the lung are those which are directed to the removal of these pathological conditions if they exist, or to the prevention of their occurrence if they do not already exist. Due attention must therefore be paid to the hygiene of the mouth, carious teeth must be removed or stopped, pyorrhœa alveolaris treated by a competent dentist, infective conditions of the tonsils and naso-pharynx dealt with adequately, the surgeon's help being requisitioned if necessary, sufficient airway ensured in the nasal passages, and the various catarrhal coriditions attended to. So much importance do I attach to the observance of these points that I make it an invariable rule to refuse to undertake the treatment of any tuberculous subject who declines the prior adequate treatment of any of the above conditions. It is little use trying to eradicate a $M$. catarrhalis infection of the lung when a condition of follicular tonsillitis in which this organism is concerned is allowed to exist unchecked, or to eradicate a streptococcal infection when from acutely pyorrhœic foci fresh streptococci are being constantly outpoured to re-infect the pulmonary tissues. No member of the community, and above all one predisposed to phthisis or the victim already of an early attack, should omit to have periodical examinations made as to the condition of the oral hygiene. Ensure healthy mouths and stamp out catarrhal attacks and mixed infections of phthisis would be no more. The former desideratum is easily attainable, but how are catarrhal infections to be obviated? It is hardly possible for us all to reside in polar regions, where they tell us such things are unknown, or even to reside under ideal conditions of air, sunshine and food. If adequate airway be ensured and correct methods of breathing taught a considerable step is taken towards prevention, but this does not suffice for a very large proportion of mankind: susceptibility to catarrhal attacks seems to be an almost universal prerogative. Some, however, are more susceptible than others. To the majority the usual catarrhal attack may be but an inconvenience- 
sometimes it is more, and means an acute pneumonia or a chronic bronchitis, but to the phthisic it is an event of the utmost importance. Life under ideal conditions does not suffice, although by toning up the system generally it certainly does tend to mitigate the severity of the attack, but something more than this is necessary; any procedure which aims at less than total prevention is inadequate.

But can this be done? I think it can. I have now had nearly ten years' experience in the production of immunity against catarrhs, and there are now many hundred cases which have either been immunised by myself or according to my directions. When I state that, so far as I can ascertain, the result among those with whom my instructions are carefully followed, is that in 90 per cent. at least 80 per cent. of the catarrhal attacks from which in previous years they suffered are completely obviated, I believe I am understating the benefit derived from this treatment. In many cases complete immunity is secured. It is hardly, however, within the scope of this chapter to enter into a full discussion of the procedure and its results in regard to the susceptible members of the human race in general; it is necessary to consider merely the procedure to be followed in those already the victims of pulmonary tuberculosis. Not only in the United Kingdom but also in other parts of the world there seem to be two severe and one mild epidemic of so-called colds annually. In England the severe ones come about the middle or end of October, and the middle of February or beginning of March : the mild one occurs at about the height of summer.

Such immunity as is to be secured by preventive inoculation can be obtained in about sixteen days, and lasts according to the individual for four to eight months. If a catarrhal attack is to be forestalled it therefore follows that treatment should be commenced at the beginning of October, February and July. The appropriate dosages, administered at intervals of about seven days, are as follows:

\begin{tabular}{|c|c|c|c|c|c|}
\hline septus. & Pneumococcus. & B. influenza. & $\begin{array}{l}\text { M. catar- } \\
\text { rhalis. }\end{array}$ & $\begin{array}{l}\text { 11. para- } \\
\text { tetragenus. }\end{array}$ & $\begin{array}{l}\text { Strepto- } \\
\text { coccus }\end{array}$ \\
\hline IOO & 50 & IOO & $5^{\circ}$ & 50 & $5^{\circ}$ \\
\hline 250 & IOO & $25^{\circ}$ & IOO & IOO & IOO \\
\hline 500 & $25^{\circ}$ & $500-1000$ & 250 & $25^{\circ}$ & 250 \\
\hline
\end{tabular}

As a condition of lowered immunity sets in within an hour or two and persists over a period of I2-I 8 hours it is obviously the best plan to perform every inoculation either when the patient is in bed or on the way home, in which latter case he should dine lightly and retire early : if rest in bed till the following mid-day be possible so much the better, but this is not an absolute essential. I, however, advise a 
little extra care in avoiding damp, cold and draughts during the twentyfour hours ensuing upon an inoculation.

During the past three years of the fifty tuberculous cases which I have either treated myself or of which I have directed the treatment, only four, I believe, have contracted a single catarrhal attack, and each of these had through force of circumstances failed to undergo immunisation for over six months-in each case the infection was a pneumococcal-influenzal one, and the highest dosage of influenza vaccine which any of them had received was 250 millions. Now I consider that full immunity against the influenza bacillus cannot be secured with dosages of less than 500 millions, and that those of rooo millions are even preferable. A little malaise, quickened pulse, rise of a degree in temperature and perhaps a little headache, all passing away within eighteen hours, are the only ill-effects I have ever seen result from this procedure.

Upon three occasions I have had patients at sanatoria at which there occurred severe catarrhal infections; the reports were made to me that my patients were the only ones that failed to be attacked.

It will be observed that in the vaccine $I$ have recommended above no B. of Friedlander is included: the reason for this is that this organism has not appeared in England for some years to give rise to catarrhal epidemics; for the time at least its energies are in abeyance ; and it is perhaps as well to stimulate the immunising mechanism of the tuberculous in as few directions as possible. For this latter reason also a slightly modified programme may be adopted, viz. : for those who are concerned with their care to keep themselves well informed of the onset of catarrhal epidemics and of the precise bacteria to which they are to be attributed and to have the appropriate mixture of vaccines at once compounded and utilised in the immunisation of their patients. This procedure has the merit of exciting the formation of as few specific anti-bodies as possible, but it has these demerits: (I) a catarrhal attack may forestall knowledge of its impending presence; (2) adequate immunity is certainly not to be anticipated from the first inoculation, and hardly from the second; the slight increase in immune bodies due to the first inoculation may not suffice to ward off an attack. It is perhaps best, therefore, to err on the side of safety and follow closely the procedure first described.

Should, despite all precautions, a phthisical individual contract an acute catarrh, bronchitis or pneumonia, it is obviously advisable to limit its severity as much as possible. This is best done not only by employing such general measures as experience has shown to be of use, but also by subjecting the patient to a course of specific immunisation with autogenous vaccine. Here I should again mention the 
extraordinary tendency of certain organisms, notably the pneumococcus, B. influenze and some strains of streptococcus, to persist in the lung tissues long after all symptoms have apparently disappeared. This is true both for the tuberculous and the non-tuberculous, but especially for the former, and to these it is a matter of even greater moment than to the latter. The way in which these organisms may persist is well illustrated by two recent experiences of mine. A patient was sent to me by Dr. H. O. Brookhouse, the symptoms complained of being recurrent pyrexial attacks of short duration, accompanied by profound depression and a tendency to faint; between these attacks the patient was apparently perfectly well. The only clue to the mystery, for several well-known consultants had failed to diagnose the case, was afforded by a history of a right-sided pneumonic attack twelve years previously. This time corresponded with the onset of a severe influenza-pneumococcal epidemic, and as I was well conversant with the tendency of these bacteria to take up their permanent abode in the pulmonary tissues, I made search for their resting-place, and finally detected an area about as large as an half-crown piece, just external to and below the right nipple, where there was harsh breathing and an occasional râle; lung puncture was resorted to, 2 c.c. of broth introduced into the lung tissues and part of it aspirated away again. From this fluid pure cultures of the pneumococcus and B. influenzac were obtained, and vaccines of these made and utilised in treatment. It is yet too soon to be certain of the ultimate effect as only about a year have elapsed since treatment was begun, but Dr. Brookhouse has recently informed me that so far there has been no recurrence.

Another case was that of an old man, aged 84 years, very hale and hearty, but the victim to every catarrhal epidemic which arose. These always led to a fortnight's confinement to bed and a further month of convalescence. I was called in to see him three years ago, and found the chief invader to be the pneumococcus, the M. catarrhalis being also present. I made vaccines and gave him an inoculation of 50 millions of each, with the result that he was out walking in the sunshine upon the third day. Treatment was continued for six months, as careful examinations of the scanty sputum shewed the continued presence of the pneumococcus. The patient then went to Biarritz, where Sir James Reid kindly continued the inoculations for me. When the patient returned two months later, he was very well indeed, but in the lump of sputum which he voided daily on waking, pneumococci still were present. Treatment was continued at intervals of about four months, the result being that in all the three years the patient has completely escaped every catarrhal epidemic, despite his advanced years, and the fact that he now winters each year in England till the 
middle of February. When last I saw him the pneumococci still were present, and I strongly urged upon the patient the necessity of continuing with periodic inoculations. I told him that it was only by persistent treatment at eight- to ten-day intervals that a reasonable expectation of freeing him from his enemies could be held, and that as this was hardly practicable the only alternative was to maintain his resistance at a high level by occasional inoculations.

I could cite several other cases to illustrate this point, but these two must perforce suffice.

The importance of this observation in the treatment of a patient who contracts an influenza-pneumococcal cold is obvious; vaccine treatment must be continued at regular intervals until repeated bacteriological examinations have revealed the complete rout of the invaders, or if this cannot be secured-and this unfortunately will surely prove to be so in a certain proportion of cases-a short series of inoculations at intervals, say, of three or four months, must on no account be omitted; in this way acute exacerbations may be obviated.

The favourable experiences which have resulted in my hands from the use of the measures described in this section have been corroborated to a certain extent by those of others. Thus Webb in Kleb's Tuberculosis, p. 590 , says :

"At the time of writing 45 patients undergoing inoculation with tuberculin and mixed vaccines have been exposed to a very wide-spread epidemic of a catarrhal condition, due chiefly to the $B$. influenza, partly to $M$. catarrhalis. Of these 45 patients, 5 had the influenza bacillus persistently in the sputa, and they have been inoculated periodically with their influenza vaccine. These have escaped colds, The results are only suggestive; they may be tabulated as follows:

$$
\text { Particulars. }
$$

"Inoculated against influenza with their own vaccine.

"Inoculated against influenza with a stock vaccine.

" (All these gave accurate histories of repeated former attacks.)

"Preventive inoculations not given, patients denying that they ever had influenza.
No. Result.

Escaped epidemic.

I 5 contracted colds and died.

"In the sputa of all I5 who died the B. influcnze was found: two of these for the first time developed very serious hæmorrhages within a few days of the infection."

He states that he has observed a patient's opsonic index to the 
tubercle bacillus lowered to 0.6 during an influenzal attack and continues :

"It is a common observation that patients with pulmonary tuberculosis may go rapidly down hill following an attack of influenza, and the writer would suggest that the prevention of such concomitant infection should be sought by the means indicated above."

On page 599 he says-" A lasting immunity must not be expected from inoculations by these vaccines, just as a lasting immunity to the tubercle bacillus is unattainable by any tuberculin administration. After the apparent maximum results have been obtained most patients have been kept in touch by inoculation at intervals of a fer weeks."

Powell and Hartley (Diseases of the Lungs, p. 5I 4 ), say: "In some cases in which there is an inveterate tendency to recurrent catarrh, whether influenzal or otherwise, it may be thought desirable to ascertain the organism of infection- $M$. catarrhalis, $B$. cory a segmentosus, $B$. influenza, the pneumococcus or other-and to prepare a vaccine to be used at appropriate intervals, with a view to increase the resistance to these organisms, and thus ward off the attack."

Methods other than Specific employed in the Prevention and Cure of Concomitant Infections.

Of the methods other than specific which are available for the prevention of mixed infections in phthisis, not much remains for mention; they may all be included in the phrase "the general hygiene of the body." More and more is it forced upon our notice that bacterial invasions depend upon a lowering of the general vitality of the tissues, for upon their vitality the formation of sufficient antibodies in part depends. Many members of the community appear to be peculiarly susceptible to the attacks of one or more bacterial enemies, and, as has been amply demonstrated during the past few rears, therapeutic immunisation with the corresponding bacterial emulsions will do much to render them immune. Unfortunately this procedure, admirable as are the results it frequently secures, sometimes fails, and this failure is due to the following defect in the method: it does little or even nothing to remedy permanently the ingrained and perhaps congenital defect on the part of the tissues in the elaboration of the specific antibodies. It supplies a stimulus, but the effect of all stimuli wears off in course of time unless a constant vis à tergo is maintained: constant stimulation may perhaps train the tissues in the way in which they should go, but given an inherent weakness on their part to make response beyond a certain point, and constant stimulation can only end in exhaustion more or less complete. 
Herein lies the danger in all forms of specific therapy, and this danger can only be avoided by the simultaneous removal, more or less complete, of the intrinsic defect in the tissues. Our ignorance of the minute mechanisms of the body and of the precise factors concerned in the maintenance of immunity are serious-obstacles to the securing of the desired result. Clinical experience, however, clearly indicates that a healthy condition of the tissues is only to be maintained by the strict adherence to certain laws of hygiene. These are so well known and so fully described in all treatises upon the diseases of the chest that there is little need for my dwelling on them here. I have already emphasised the necessity of dealing with all infective foci in the body generally, and so of removing all sources of toxic absorption which impair the general nutrition and the resisting powers of the tissues devoted to the elaboration of the immune bodies, and of securing proper freedom of the air-passages and of inculcating correct methods of respiration. A pure and adequate supply of air must be ensured, proper food in adequate amounts must be supplied, and its utilisation made possible by attention to the organs of mastication, to the stomach and the bowels. If necessary the appetite must be stimulated by the use of suitable tonics. Mental quietude is a desideratum for all, and suitable exercise an important essential. Finally isolation as far as possible of all those who suffer from infectious diseases, and into this category catarrhal attacks most certainly fall, and their adequate treatment cannot fail to remove an important potential source of danger to others.

These are the general prophylactic methods whereby the incidence of mixed infection may be diminished in the phthisical. It remains to consider the methods other than specific which may be utilised in the treatment of mixed infection when it has already supervened.

Absolute rest in bed until the pyrexia is under control and open-air lines of treatment cannot be too strictly enforced; an abundant and well-assorted dietary rich in fats and nitrogenous elements and conducive to their retention in the body is essential; plenty of good milk and cod-liver oil with the addition perhaps of a little strychnia, iron or arsenic being included if possible; while various forms of drug treatment have been devised for dealing with the mixed infection. These comprise : (I) antiseptic inhalations; (2) intra-tracheal injections; (3) drugs by the mouth; (4) subcutaneous and intra-venous inoculations; (5) applications from without; and will be considered in order.

To Dr. Churchill may be justly attributed not only the introduction of hypophosphites in the treatment of pulmonary tuberculosis, but also of inhalant mixtures for the combat of mixed infections. His so-called spirone was a mixture of iodine, potassium hyposulphite 
and acetone, and was much belauded by its inventor; recent analyses performed at the Lister Institute and Clinical Research laboratories tend to show that it is practically devoid of antiseptic properties, containing only a solution of potassium iodide and potassium tetrathionate in acetone.

The use of inhalations passed somewhat out of vogue, but has been continued by Drs. Burney Yeo, David Lees, Muthu and others, and its proper importance been assigned to it. The absolute necessity of continuous inhalation, not of one extending over merely a few hours daily, has been demonstrated. As the treatment is apt to prove irksome to the patient the use of specially light but well-fitting respirators is very advisable. Messrs. Maw and Sons manufacture a form devised by Dr. Burney Yeo consisting of perforated zinc bound round the edge with chamois leather, and furnished with a small receptacle for the cotton-wool to which the respiratory mixture is added.

Various mixtures for inhalation have been devised; the one most in use at Brompton Hospital is one composed of equal parts of tincture of iodine, liquefied carbolic acid, creosote, ether and rectified spirit; another is menthol $j^{\mathrm{ij}}$, creosote $j^{\mathrm{iss}}$, spiritus camphoræ $j^{\mathrm{iss}}$, spiritum rectificatum ad $3 \mathrm{j}$. Twenty drops of either of these are to be employed hourly to moisten the cotton-wool inside the respirator.

Some mixtures suit certain patients better than others: for instance when the larynx is involved one containing menthol seems to give the best results, when there is troublesome laryngeal cough the addition of chloroform appears to be indicated, while the addition of $j \mathrm{j}$ of oil of bitter almonds to an ounce of mixture seems of especial service to some. If one mixture does not suit, trial should be made of another.

Personally I have been disposed to regard antiseptic inhalations as having little direct influence upon the mixed infections of phthisis, and to attribute their undoubted good effects to the diminution of cough and the increased comfort of the patient. Others whose experience is far greater than my own insist upon their sterilising action; among these I may mention Dr. David Lees and Dr. Burney Yeo.

Intra-tracheal injections. - Intra-tracheal injections have found especial favour abroad, and have been advocated in this country by Sir T. Grainger Stewart, Mr. Colin Campbell and others. By means of a special form of syringe and a certain amount of practice these may be made with little inconvenience to the patient. A drachm or two of one of the following mixtures should be injected once or twice daily : menthol Io parts; guaiacol 2 parts; olive oil 88 parts, or gomenol 5-IO parts, olive oil to Ioo parts. Suspensions of iodoform in olive oil have been similarly employed. This method of treatment appears to be of especial service in cases of advanced cavitation, but the results 
obtained vary greatly from almost complete failure to a considerable measure of success.

Drugs by the mouth. - The drug treatment by the mouth of the mixed infections of pulmonary tuberculosis can hardly be anticipated to produce any marked bactericidal or inhibitory effect unless persisted in for a considerable period. It has also the additional disadvantage that it may tend to upset the digestive apparatus. Drugs by the mouth also require to be employed with considerable discretion: a preparation well suited for use in one class of case may be inadvisable in another class of case. For instance, when there is little or no pyrexia but considerable expectoration, the preparations of tar, creosote or eucalyptus in moderate doses may prove of service. The tar or creosote may be given as a pill, five or ten drops of eucalyptus oil and terebene in equal parts may be taken on sugar, while tar is best given as a morning and evening dose of eau de goudron, $5 \mathrm{j}$, in a little warm milk to which a teaspoonful of brandy may be added. Creosote and guaiacol may be given in 2 to $4 \mathrm{~m}$ doses inside a perle or capsule. These preparations all act through their excretion by the bronchial mucous membrane, whereby suppurative processes in the walls of cavities and around tuberculous foci is limited. When it is remembered that suppuration is an important means whereby necrotic tuberculous tissue is eliminated, explanation is afforded of the clinical fact that remedies of the creosote class prove of very doubtful service during the acute and actively pyrexial stages of phthisis, and are especially indicated as the pyrexia is subsiding and the more quiescent period entered on.

In the former stage, therefore, the object of drug treatment is rather to be directed toxvards the limitation of toxic absorption by the control of excessive coughing and the promotion of easy expectoration rather than to the limitation of the sputum.

In the chronic fibroid stage drugs may be employed with greater freedom according to the nature of the cough and the amount of the expectoration.

Subcutaneous and intra-venons inoculations. - The subcutaneous and intravenous introduction of antiseptic drugs with the object of a "sterilisans magna" has been repeatedly advocated. Numerous drugs such as formalin, iodoform, mercury succinimide, quinine derivatives, creosote and guaiacol either as aqueous solutions or as oily suspensions have been employed, and beneficial results recorded. The destruction of the tubercle bacilli in situ has been the object aimed at; this, it may at once be said, is an absolute impossibility: for the destruction of the tubercle bacilli such a dose of antiseptic would have to be given as would necessarily hasten considerably the patient's end. It is hardly even likely that the death of the much more easily destructible secondary 
invaders has been accomplished. Such a lowering in their vitality may, however, have been produced as would enable the tissues of the lung the better to grapple with their enemies. The discovery by Ehrlich and Hata of drugs which have a selective action, combining with certain protoplasm but not with others, affords hope that the discovery may yet be made of drugs which have a special affinity for the tubercle bacillus, pneumococcus, $B$. influenzer, etc. Morgenroth claims to have found such an one for the pneumococcus in a certain complex quinine derivative. Sir Almroth Wright confirms its selective action and has found that it will destroy pneumococci in the blood-stream; its practical utility in cases of pneumonia he has disproved, and it is hardly advisable to be unduly optimistic of success by any method of "sterilisans magna."

Regarding the use of intra-muscular injections of creosote or guaiacol dissolved in olive oil, Powell and Hartley say: "We have had opportunities of noticing the results of this method of treatment, but have by no means been convinced of its superiority (i.e. to mouth medication); the imagination of the patient cannot fail for a time to be impressed, but at the cost of considerable discomfort and in some instances of no little positive suffering, local necrosis sometimes occurring at the seat of injection."

Applications from without.-The application of strong iodine preparations or of blisters, while yet useful in the treatment of quiescent cavities, can hardly be expected to produce any direct influence upon the secondary infection.

The utility of radium applications in the treatment of bacterial diseases has been so much to the front of late that the question at once occurs whether they would prove of any avail in the treatment of the condition with which, we are concerned. No authoritative and authentic statements on this point have yet occurred in print. I have, however, heard privately that a radium corset has been devised completely enveloping the chest, and that the application of this for one hour daily has been productive of some good results. Further information upon this subject will be awaited with much interest, but the method obviously can hardly become one of general applicability. 


\section{CHAPTER XI.}

\section{INFECTIONS BY THE TUBERCLE BACILLUS AND THE USE OF SPECIFIC PRODUCTS IN THEIR TREAT- MENT.}

In the preceding chapter I have endeavoured to bring before the reader the paramount importance of mixed infection in many, if not most, cases of pulmonary tuberculosis, and the necessity of coping adequately with these allied invaders before endeavouring to secure the extinction of the infection by the $B$. tuberculosis itself. It now remains to consider how this latter objective may be attained, and this question I propose to discuss according to the following scheme:

(I) The tubercle bacillus group. Its distribution in nature-paths of infection-constitution of the bacillus, its toxins, and their actions on the tissues. The defensive mechanism of the body and how this may be assisted generally.

(2) The tuberculins and their use in the diagnosis of pulmonary tuberculosis. "Allergie" or reactivity-cutaneous test of Von Pirquet - percutaneous test of Morro-Carle Woodcock blister test-intradermal test-ophthalmo test of Wolff Eisner and Calmette-the subcutaneous test-opsonic index estimations.

(3) Classification of cases of pulmonary tuberculosis according to (a) extent and nature of lesions, (b) auto-inoculatory phenomena-the nature of the immunity that can be secured by means of tuberculinselection of cases for specific therapy.

(4) Choice of tuberculin-conduct of course of treatment-control of dosage and intervals.

(5) Treatment by the induction and control of auto-inoculations.

(6) Results.

The Tubercle Bacillus Group-Its Distribution in Nature-Paths of Infection in Man-The Constituents of the Bacillus, its Toxins, and their Actions on the Tissues.

As we have seen on p. I9, the B. tuberculosis belongs to the group of bacilli known as acid-fast, which also includes the smegma bacillus, 
B. lepra, B. phlei, or Timothy grass bacillus, the Mycobacterimm lacticola, or butter bacillus of Rabinowitsch, and other little-known organisms. These are now regarded as being more correctly designated as mycobacteria, or even as streptothrices.

While it is true that the B. phlei and Mycobacterium lacticola grow readily at a temperature of $22^{\circ} \mathrm{C}$., this fact, contrary to the usual statement, does not suffice to differentiate them from all varieties of the $B$. tuberculosis, for it is now known that such cold-blooded animals as the tortoise, frog, blindworm and fishes are sometimes infected by varieties of the bacillus, morphologically closely resembling the varieties which infect the warm-blooded animals. Man, cattle, swine, horses, monkeys, rabbits, guinea-pigs, dogs, cats, birds, and, in fact, most warm-blooded animals, are susceptible to the attacks of the $B$. tuberculosis, and considerable investigation has been conducted upon the identity or otherwise of the several varieties of the bacillus. The discussion has waxed exceedingly hot in regard to the human and borine bacilli, but the consensus of opinion now is that the two are distinct varieties, and are not convertible the one into the other by even very prolonged residence in the tissues of the alien host.

In each species pathological conditions can be initiated by the introduction into suitable sites in its tissues of living bacteria derived from the other animal, but man is more susceptible to the attacks of the borine bacillus than are cattle to the attacks of the human bacillus. Indeed, Behring and Baumgarten have shown that under certain conditions cattle can be rendered immune to the bovine bacillus by means of inoculations with living cultures of the human strain. As we shall see later, Spengler, Klemperer, Klimmer, and Friedmann have attempted to bring about an analogous result in man with bacilli derived from various sources, but with doubtful success. The question of the infection of man by the bovine bacillus is one of immense economic importance, and has great bearing upon the means whereby tuberculosis is initiated in the human subject. It is quite impossible here to enter into a lengthy discussion of the various differences of observation and of opinion; as regards the following points there is almost general agreement:

(a) That tuberculosis in man can be initiated both by the human and borine strains, despite the failures on the part of Spengler and of Klemperer to infect themselves by subcutaneous inoculation with $\frac{1}{2}$-gr. doses of living virulent borine bacilli.

(b) That there is no very marked difference between the incidence rate of tuberculosis in countries where cow's milk is freely consumed, and that in countries where it is not used for human consumption.

(c) That there is, however, a certain difference in the nature of the 
tuberculous lesions initiated by the two bacilli, glandular tuberculosis, especially in the abdomen and less frequently in the neck, being especially common in those countries where cow's milk is freely consumed. Bacteriological examinations have confirmed the frequency of bovine infection of the glandular system in man. Only occasionally are the bones, joints, or meninges attacked by the bovine bacillus, and very rarely the pulmonary tissues. Carl Spengler has modified his original view that in pulmonary tuberculosis the two bacilli occur side by side, and now states that two types, which he calls human and bovo-human, so occur, and that the latter type is identical with Much's granular non-acid-fast variety.

\section{Paths of Infection.}

Assuming, then, that pulmonary tuberculosis is almost always spread from man to man-though personally I believe that insufficient attention is paid to the domestic cat as the carrier of infection to the children who pet and fondle them-it is necessary to consider briefly the routes whereby the bacilli may reach the lungs. Among these may be mentioned :

(a) Via the placental circulation-this is certainly very rare.

(b) Via the maternal milk-a possibility which has not, I feel, received sufficient consideration, for if tubercle bacilli are freely discharged in the milk of tuberculous corvs, whose udders, nevertheless, are perfectly healthy, it is almost inconceivable that bacilli are not likewise excreted in the milk of tuberculous mothers.

(c) By the transference of the bacilli to the mouth from the hands of children crawling about the floor of rooms frequented by a tuberculous person.

(d) Transference of the bacilli to the lips in the act of kissing, a danger which is greatly increased if a tuberculous male be allowed to retain a beard or moustache.

(e) By inhalation into the upper respiratory tract of air laden with tubercle bacilli, which may be-

(I) Caught up by the nasal mucosa, and be either destroyed there by the endothelial and lymphoid cells, or be swallowed in the excessive secretion of a nasal or post-nasal catarrh, or be expelled upon the handkerchief.

(2) Caught up by the saliva and swallowed, or they may find a portal of entry into the tonsils or adenoid tissue of the naso-pharynx.

(3) Entangled by the ciliated cells of the larynx and trachea and find lodgment there or be expectorated in the sputum.

(4) Carried down into the bronchi and bronchioles with the respiratory air and deposited in the pulmonary tissues. 
Whatever the portai of entry may be the bacilli may enter the lymphatic spaces, and possibly the blood-ressels; in the former event they are carried to the nearest 15 mph-node, where either they may be detained, or they may be allowed to pass through and even enter ultimately the general blood-stream. Thus, bacilli of the human type may be air-borne, and yet the foci of infection may be established in such diverse situations as the nose, tonsils, adenoid tissue of the nasopharynx, cervical glands, larynx, bronchial glands, pulmonary tissues, or abdominal glands.

Bovine bacilli are commonly ingested in infected milk, and either adhere to the mucous membrane of the pharynx and enter the tonsils and cerrical glands, or are absorbed through the permeable mucous membrane of the gastro-intestinal tract and infect the abdominal glands and peritoneum, but only rarely the pulmonary tissues.

\section{The Constitution of the Tubercle Bacillus; its Toxins and their Actions upon the Tissues.}

The tubercle bacillus can be readily grown under artificial conditions, although its rate of multiplication is slow relative to most other micro-organisms. The best media for the purpose are: solid media, Dorset's egg medium or glycerine agar, liquid medium, veal broth containing 5 per cent. glycerine. If the bacteria be scraped off the surface of the solid medium and be extracted with various solvents certain bodies are obtained; among these may be mentioned:

(I) A fatty acid, which confers upon the bacillus its acid-fast character and appears to hare more or less the same constitution in all acid-fast bacteria. This body is said to be the cause of the chronic inflammatory and caseous changes which occur in the tissues.

(2) Albumoses which are capable of causing rise of temperature when injected into the tissues, and are probably responsible for some of the pyrexial symptoms in the natural course of the disease.

(3) Certain volatile bodies which are said to have a convulsant action.

Renon gives the following account of the products which may be obtained with various solvents:

(I) Distilled water removes albumins.

(2) A Io per cent. solution of sodium chloride acting for twentyfour hours at $3 \mathrm{~S}^{\circ} \mathrm{C}$. removes globulins.

(3) Alcohol, ether, and chloroform remove the fatty envelope, the two former more especially that constituent which leads to caseation effects, the chloroform the body which induces sclerosis.

(t) Strong acetic acid at $\mathrm{So}^{\circ} \mathrm{C}$. dissolves the casein, a para-nucleo 
albumin, which is the essential protoplasmic substance, and which when stained with carbol fuchsin strongly resists decolorisation by means of acid, but yields more readily to treatment with absolute alcohol.

There is thus no doubt that the tubercle bacillus forms within itself bodies capable of producing definite pathological effects upon the tissues of its host, and known as intra-cellular or endo-toxins. These are not set free until the death of the bacillus and its subsequent disintegration or solution.

Whether the bacillus also resembles the $B$. diphtherice in forming poisonous substances which are excreted "in vivo" into the tissues of the host is not certain; probably a minute quantity of extra-cellular or exo-toxin is so formed, but the rate of multiplication of the bacillus in artificial culture is so slow that it is very difficult to determine with certainty whether the whole of the toxin found in the culture fluid is not simply endo-toxin set free by the death and autolysis of the bacilli. However this may be, it is certain that the rôle played by any exotoxin is of negligible importance compared with that played by the endo-toxins.

\section{The Defensive Mechanism of the Body against the Tubercle Bacillus, and how this may be assisted generally.}

The defensive mechanism of the body against the tubercle bacillus is but little understood. In the case of certain acute infective disorders such as scarlet fever, measles, and smallpox, one attack seems to confer a very high degree of active immunity which may even last a lifetime, and it is accordingly rare for the individual to be subjected to a second attack. Such does not appear to be the case with tuberculosis ; so far from one attack conferring immunity against a second it seems rather to predispose to it; that is, if we except the immunity to subsequent infection by the human strain which may possibly be conferred by an infection due to the bovine type. 'Koch thought that he detected some attempt at the establishment of immunity in cases of miliary tuberculosis, but inasmuch as these cases always end fatally, the attempt obviously is doomed to failure. At the same time, if an animal deeply infected intra-abdominally with tuberculosis be inoculated subcutaneously with virulent bacilli, a slight lesion which rapidiy heals is the sole result.

That general constitutional immunity exists, due probably to bio-chemical causes, is highly likely, but local immunity appears to be a most important defensive mechanism, as is well shown by the fact that areas of advance and of cure may occur simultaneously, not only in the same patient, but also in different parts of the same lesion, as is 
often seen in cases of lupus and of tuberculosis of the synovial membranes. Limitation of local processes is brought about by the organisation of the giant-cell systems and the formation of fibrous tissue and by the calcification of caseous areas.

Phagocytosis, apparently, is a very important agency in the destruction of the bacilli, and is performed mainly by the giant and endothelial cells and by the lymphocytes; the polymorphonuclear leucocytes are possibly of service in destroying such bacilli as may gain access to the blood-stream, but inasmuch as the ingress of any considerable numbers of bacilli into the circulation almost invariably leads to generalised tuberculosis, they obviously fail to fulfil this rôle completely.

That the amount of opsonin in the circulating fluids is of considerable moment to the activities of the polymorphonuclear leucocytes has been amply demonstrated, but whether opsonin is of any service in assisting the giant and endothelial cells in the performance of phagocytosis is not known. It is probable that the toxic products formed by the bacilli stimulate these cells to proliferate and so to grow round and enclose the invaders.

Agglutinin for the tubercle bacillus is sometimes found to be present in the serum of infected individuals in considerable quantities; in the healthy it is altogether absent, or present only to a negligible degree. Inasmuch as it may be almost altogether absent throughout the course of an attack, and yet progress to recovery be quite satisfactory, the importance of agglutinin in immunity cannot be very great.

Formation of bactericidin and of antitoxin has not been satisfactorily demonstrated, nevertheless some formation of the latter almost certainly occurs. As we shall see later, the tissue-cells respond to increasing doses of toxins, not by the elaboration of antitoxin, but by acquiring tolerance of the poison. On the other hand the importance of lysin is becoming increasingly clearer: whereas the amount of tuberculo-lysin in the tissues of the healthy individual is very small, in the case of an infected individual or of one who has been immunised with tuberculin the amount may become very considerable; this, however, is not always so, and some cases make good progress although the amount of lysin appears to be minimal throughout the course of the disease. It must also be mentioned that the tissue-cells of a tuberculous individual appear to differ from those of a normal individual in regard to the way in which they behave to the tubercle bacillus. This question will be fully dealt with under the heading of the "Tuberculin Reaction," and it must here suffice to state that to this acquired condition the term "allergie," or "hypersensitiveness," has been applied. Personally I believe that the difference in behaviour of tuberculous and of non-tuberculous tissues is entirely one of degree and not of kind, and 
that a similar difference is of universal occurrence, no matter what the variety of the infective agent may be.

This brief account will suffice to show the magnitude of the difficulties encountered in the endeavour to find an effective weapon for combating invasion by the $B$. tuberculosis.

Furthermore, the great tendency of the tissues to surround the invaders with a more or less impenetrable zone of fibrous tissue, especially well shown in cases of chronic lupus, and also in cases of chronic phthisis, and the avascular character of the tubercles, renders almost hopeless any attempt to bring about a "sterilisans magna" comparable to that which may be effected with salvarsan in infections by the Spirochata pallida. For the same reason, granted that powerful lytic or bactericidal sera could be prepared, their use would seem foredoomed to failure in most instances, while in those cases in which the lytic serum could gain access to the bacteria the inevitable result would be the rapid flooding of the tissues with massive doses of the endotoxins thereby liberated, and the probable death of the sufferer.

It would thus appear that any procedure directed to the extinction of a tuberculous infection must perforce in its action be slow and gradual, and must maintain the protective mechanism at a constant high level. The belief that this can best be brought about by a methodical and carefully conducted course of inoculation with the products of the tubercle bacillus itself is the justification for the advocacy of the routine employment of tuberculin therapy. This will require supplementing by all the various measures which clinical experience has found of profit, such as (I) an ample supply of food which is easily digestible and so capable of being readily converted to the needs of cell nutrition; (2) abundance of pure air whereby the tissue-cells may be assisted in their metabolic activities; (3) a sufficiency of sound sleep to rest the actively functioning cells, and inasmuch as weakly cells necessarily require more rest than healthy ones, the tuberculous individual requires to rest his more than the normal person; (4) for a similar reason, hyperactivity, either of the mind or body, should be avoided, especially in view of the influence exerted thereby upon the blood-supply to the tuberculous foci; (5) all toxic influences which may damage the tissue-cells must be rigidly limited, and, if possible, removed. Toxic absorption from the gums, tonsils and nose due to conditions of pyorrhoea alveolaris, follicular tonsillitis and chronic catarrh, and from the bowels, due to intestinal stasis, are especially prejudicial to the tuberculous, and should receive the strictest care and attention. Similarly toxic absorption from areas infected by the tubercle bacillus and allied invaders must be limited except in so far as it is serving a useful purpose in the production of immunity. 
The Tuberculins and their $C_{\text {se }}$ in the Diagnosis of Pulmonary Tuberculosis.

The preparations of the tubercle bacillus for therapeutic purposes are made either from the bodies of the bacilli themselves, from the fluid medium in which they have been cultured, or from a mixture of the fluid medium and of extractives from the bacilli. Their number now is very great and only the more commonly used and important ones will be mentioned.

From the culture fluid.-(I) T.O.A. is simply the fluid in which the bacteria of the human type have grown, unaltered in any way except that the bacillary bodies have been remored by filtration through a Chamberland candle.

The corresponding preparation from the bovine strain is known as P.T.O., not P.T.O.A.

(2) Tacuum tuberculin is T.O.A. which has been concentrated by evaporation at a low temperature in a partial vacuum to one tenth its original bulk. Deny's tuberculin corresponds closely to this preparation. The corresponding borine preparation is designated bovine vacuum tuberculin.

(3) T., or old tuberculin, is prepared from a four to fire weeks' old culture of the human type bacillus in glycerine broth in the following way: The cultures are treated with steam for half an hour to insure sterility; they are ther placed in steam-heated ressels fitted with a racuum apparatus, and eraporation is conducted at $70^{\circ} \mathrm{C}$. until the rolume is reduced by nine tenths. Filtration through porcelain candles is then performed, the filtrate allowed to cool, and $0^{\circ} 5$ per cent. phenol added. Sedimentation of indifferent substances is allowed to proceed in a cool place for sereral weeks: these are remored by filtration, the clear filtrate now constituting old tuberculin or $T$., which differs thus from T.O.A. and vacuum tuberculin not only in the fact that concentration has been produced with the aid of heat, but also in that the bacilli have been partly extracted with hot steam. the albumin and some of the globulin of the bacillary bodies thus being taken into solution.

The corresponding bovine preparation is known as P.T.

From the bodies.-(I) T.O.: The bacilli are finely triturated and extracted with water until all the constituents soluble therein are removed; this solution of the soluble constituents was designated T.O. by Koch, and regarded by him as of no therapeutic value. It is supposed to contain nothing but toxin. As we have already seen, the extracted substance is an albumin. (Note that P.T.O. is the borine 
preparation corresponding to T.O.A., and not to T.O., as might well be imagined.) The bacillary residue, after the extraction with water is complete, is utilised for the preparation of T.R.

(2) T.R.: The triturated bacillary residue is alternately treated with water and centrifugalised; after each centrifugalisation the supernatant fine suspension is pipetted off. These emulsions are finally bulked together, and concentrated by the aid of heat and 20 per cent. glycerine added to constitute T.R. The amount of solid matter in each cubic centimetre is derived from an initial Io mgrm. of tubercle bacilli, and the concentration is conducted until $2 \mathrm{mgrm}$. of solid substance are present in the emulsion. T.R. is thus composed of such portions of the bacillus as are insoluble in water, i.e. of some of the globulin, of the fatty envelope, and the essential protoplasmic casein or para-nucleo albumen.

The corresponding bovine preparation is designated P.T.R.

(3) Vacuum T.R. is precisely similar in method of preparation to T.R., except that the necessary concentration of the bulked suspensions is conducted in a vacuum instead of by the aid of heat.

(4) B.E., or bacillary emulsion, is prepared by triturating the whole bacillary bodies, and emulsifying each $0^{\circ} 5 \mathrm{grm}$. of the powder with a mixture of 50 c.c. glycerine and 50 c.c. distilled water. Each cubic centimetre therefore contains $5 \mathrm{mgrm}$. of bacillary substance, from which nothing has been removed by the aid of solvents.

The corresponding bovine preparation is designated P.B.E.

(5) S.B.E.: Sensitised bacillary emulsion, or tuberculosis serovaccine, is prepared as follows: Bacilli of the human type are collected on a filter, and thoroughly washed with normal salt solution. They are then placed in a suitable quantity of a specific tuberculosis immunising serum derived from an animal which has been immunised with living human-type bacilli and triturated by agitation with beads; the disintegration of the bacilli is assisted by heating the mixture of bacilli and serum together at $37^{\circ} \mathrm{C}$. for a long time. When the trituration of the bacilli is complete the mixture is centrifugalised, the sediment repeatedly washed with normal salt solution, and finally emulsified with 50 per cent. glycerine solution, I c.c. of the emulsion containing $5 \mathrm{mgrm}$. of solid matter. The idea of this preparation was that a fixation of the specific immune bodies, present in the serum of the immunised animal, should be effected with the tubercle bacilli. The idea does not seem to be realised in actual practice, despite the favourable reports of Citron.

From the culture fluid and extractives from the bacilli.-T. Bk. or Béraneck's tuberculin differs from the preceding preparations in that it is composed not only of the toxins diffused into the culture medium, 
but also of substances extracted from the bacillary bodies by the aid of orthophosphoric acid. With the aim of keeping the product as free as possible from toxic but non-immunising substances, the broth in which the bacilli are grown contains neither peptone nor albumose. When free growth has occurred the bacilli are removed by filtration, and an extract of the bacillary protein made with the aid of a I per cent. solution of orthosphoric acid; equal portions of this extract and of the culture medium which has previously been evaporated down at a low temperature in racuo to a tenth of its bulk are mixed together to form the finished product. Béraneck claims that this preparation is not only as potent as any other preparation in stimulating the formation of immune bodies, but that it is also much less toxic, so that more powerful immunising effects can be produced without giving rise to undesirable reactions. Sahli supports this contention; but from the description of some of the reactions obtained in his cases, it would seem that its toxicity for tuberculous guinea-pigs is no measure of its toxicity for tuberculous human beings. Nevertheless, there is something to be said theoretically in its favour, and further clinical experience may confirm the claims made on its behalf.

Landmann's tuberculol is somewhat similar and is prepared as follow's: Broth cultures of highly virulent bacilli after being freed from fat and finely powdered are extracted with normal saline or dilute glycerine for some time at $40^{\circ} \mathrm{C}$; the residue is repeatedly extracted with fresh fluid at a temperature which is gradually raised to $100^{\circ} \mathrm{C}$. The extracts are mixed and the total product concentrated in iacuo at $37^{\circ} \mathrm{C}$. This is then added to the culture fluid which has been concentrated in vaculo as far as possible and the whole filtered through porcelain candles: carbolic acid is then added to make 0.5 per cent., and the preparation is diluted until I c.c. just suffices to kill a guinea-pig weighing $250 \mathrm{grm}$. This preparation has the advantage of accurate standardisation of its toxin content; the disadvantage, according to Béraneck, of not only containing much non-specific toxin but also of containing an undue amount of specific toxin relative to its power of exciting immunising response.

The doubt existing as to whether the strains of tubercle bacilli infecting man are identical in every instance, or whether they merely closely resemble each other as do the various streptococci, has led to manufacturers employing cultures of strains derived, not from one, but from several sources; to the preparation derived therefrom the term "polygenous" has been prefixed, viz. polygenous bacillary emulsion. This I have long advocated and am convinced is a step in the right direction.

Numerous attempts have also been made to bring about active 
immunisation on lines parallel to those introduced by Jenner for smallpox and by Pasteur for rabies and anthrax. To this end bacilli either of attenuated virulence or devoid of pathogenic power for man have been employed by Klimmer and by Friedmann. Klimmer utilises human bacilli which have been completely deprived of their infecting power by long heating to $52^{\circ} \mathrm{C}$. or by passage through animals; these, he states, do not regain their virulence when introduced into the human tissues. Friedmann has just introduced an avirulent preparation derived from a tuberculous giant tortoise and claims the most striking successes. His claims, like those of Klimmer, require further confirmation; on mere à priori reasoning they seem likely to require considerable qualification.

\section{The Tuberculins in the Diagnosis of Pulmonary Tuberculosis; "Allergie" or "Reactivity."}

Whereas doses of I c.c. or even 5 c.c. of old tuberculin have been introduced subcutaneously into the tissues of an individual entirely free from active tuberculosis without producing any appreciable disturbance, constitutional or otherwise, quite otherwise is it with infected individuals, in whom relatively minute doses act as a powerful poison and produce a train of characteristic symptoms. Infection has brought about a condition of their tissues not present in those of the healthy, a condition to which the terms "allergie," "reactivity" or "sensitiveness" have been applied. In the healthy the albumin of the tuberculin is dealt with like any other foreign albumin, and a condition of anaphylaxis can be induced if the initial dose of tubercular endoplasm be sufficiently large. In the infected it is dealt with apparently in quite another way: the presence of living tubercle bacilli in the tissues has brought about an obscure change in the cells whereby they are enabled to break down, more or less slowly, the albumin of tuberculin into a toxic and a non-toxic portion. Wolff-Eisner has suggested the term "tuberculo-lysin" for the unknown substance whereby this is brought about. The theory is not an entirely satisfactory one, but it is simple and explains most observed facts. All the methods wherein tuberculin is utilised for the diagnosis of tuberculous infection depend upon the presence in the tissues of this hypothecated tuberculo-lysin, which, as has been stated, is only formed when living tubercle bacilli are resident in the tissues.

The cutaneous test of von Pirquet is performed as follows: The skin on the inner side of the forearm is rubbed with ether, one drop of a 25 per cent. dilution of old tuberculin is placed thereon, and 4 in. below it a drop of undiluted tuberculin. By means of a platinum inoculation needle a circular scarification, just penetrating the epidermal layer as 
in ordinary vaccination, is made midway between the two drops. The operation is then repeated on the skin underlying the 25 per cent. dilution, and lastly, at the site of the undiluted drop. After the inoculation the tuberculin is allowed to soak into the tissue spaces for some minutes or a minute piece of cotton-wool is applied to each area, no other dressing being required. Inspection is to be made at the end of twenty-four and forty-eight hours, and if no reaction be then apparent again at seventy-two and ninety-six hours. In interpreting the results it is necessary to distinguish between-

(I) The traumatic reaction due to the scarification.

(2) The negative reaction.

(3) The positive reaction.

The traumatic reaction begins within a few minutes at each locality; at first it consists of a small raised area surrounded by a red areola, which disappears within a few hours; there remains only a slightly raised redness, with a papule, about as large as a pin's head, in the centre. After twenty-four hours only a little redness in the immediate area of the scarification is to be seen; this soon disappears, a small brown scab remaining for the next few days.

In the case of negative reactions an appearance similar to that at the control area is presented; the distinction from minimal specific reactions is by no means easy, and ron Pirquet advises that reactions under $5 \mathrm{~mm}$. in diameter should be regarded as doubtful, and definite judgment suspended until repetition has been made, as thereby the signs of a positive reaction are accentuated.

In the positive reaction there is a latent period varying from three hours to several days, during which nothing more than the corresponding traumatic reaction is to be seen. In most cases it is well developed after twenty-four hours and most distinct at the end of forty-eight hours; if not seen till later it is called "torpid." It begins as hyperæmia and exudation, and appears as a slight red swelling, commencing in the punctures, and rapidly developing in area and height; the edge of the papule is sometimes rounded, sometimes indented; occasionally it is surrounded by a flat, slightly bluish areola, from the margin of which reddened lymphatics may be traced. As a rule the papule measures about ro mm. in diameter, occasionally $20 \mathrm{~mm}$, and rarely in cases of rery severe reaction $30 \mathrm{~mm}$. The maximum reaction is usually attained in forty-eight hours : the exudation then begins to decrease, the redness fades, passes into riolet and gradually into a pigmentation which may persist for weeks; the epidermis usually peels slightly. Rarely the papule is colourless and can only be felt.

A positive reaction is not accompanied by any rise of temperature or 
other constitutional disturbance, and if the test is properly conducted never gives rise to any complications or sequelæ. It gives indication of a specific tuberculous infection, but gives no information as to the nature or site of the lesion, of its activity or quiescence; it only indicates that infection has occurred at some time and place.

A negative reaction is strong evidence of the absence of tuberculosis, but is sometimes seen in the last days of a fatal tuberculosis, in the final stage of miliary tuberculosis or tubercular meningitis, occasionally in tuberculosis complicated by some other disease, and in advanced cachectic conditions.

Inasmuch as over go per cent. of all adults have been infected with tuberculosis at some period or other of their lives the diagnostic significance of the test is very greatly reduced, and its value as an indication of active tuberculosis can only be accepted in children up to the age of three or four years, in whom healed tuberculosis is a rare phenomenon.

The attempt has been made by Sahli and others to so modify the test as to enable it to be employed as a measurement of the sensitiveness or reactivity of the tissues, and so as an indication of the appropriate dosage of tuberculin for therapeutic purposes. To this end the test is made in turn with dilutions of $I$ in IO, I in IOo, I in Iooo, and if necessary I in I0,00o, that dose being considered to be the one suitable for subcutaneous use which corresponds to half that minimal quantity which gives a positive reaction.

Morro's percutaneous test is conducted as follows: The skin on the inside of the forearm or just below the tip of the ensiform cartilage is rubbed well with ether. A piece of ointment as large as a pea, prepared by mixing thoroughly together at a temperature of $20^{\circ} \mathrm{C}$. equal parts of lanoline and old tuberculin, is then rubbed in with moderate pressure by the finger-tip for half to one minute over an area of about $5 \mathrm{~cm}$. diameter. As with the cutaneous test so different grades of reaction are presented by the percutaneous test, varying from the appearance after twenty-four to forty-eight hours of isolated reddened spots to papules indistinguishable in size and appearance from those produced by the von Pirquet test. As a rule, a number, twenty to thirty, of discrete miliary nodules with reddened areola are produced, accompanied by itching and lasting for several days, after which there is some pigmentation and peeling of the skin. Of the specificity of the test there is no doubt, but its reliability is distinctly inferior to that of the cutaneous test.

De Carle Woodcock blister reaction is a modification of Von Pirquet's test. Two small blisters are applied to the skin of the back, of the chest, or other locality. When the skin has risen it is removed 
with scissors and a water dressing applied for twenty-four hours to remove the hyperæmia. To one of the denuded areas a drop of undiluted T.R. is applied and allowed to dry-this takes about half an hour; a dressing of dry lint is then applied to both areas, and these are compared after twenty-four hours. If tuberculosis be present, the surface treated with tuberculin is then rosy red with a blush on its circumference, and there is some exudation; the untreated surface is comparatively pale. The test is more complicated, occupies more time, and appears to possess little advantage over the von Pirquet test.

The intra-dermal test of Mantoux.-A solution of $I$ in 5000 of old tuberculin, to which stovaine to make $0^{\circ} 5$ per cent. is added in order to diminish the resultant pain, is employed for this test. The skin is washed with alcohol or ether, and by means of a fine short needle I drop, or $0^{\circ} 05$ c.c., of the solution is introduced, so that it lies just beneath the epidermis and over the hypodermis. In order to secure this result the syringe must be perfectly air-tight, the exit of the needle point must be turned towards the surface of the skin and the fluid gently expressed; it should then be apparent as a little drop of cedema about $4 \mathrm{~mm}$. in diameter.

A positive reaction is indicated by the appearance after four to eight hours of an infiltration, white or rosy red in colour. This rapidly extends, and at the end of twenty-four to forty-eight hours, when the reaction is at its height, the infiltrated area is rosy or bright red, rarely pale, and is $\frac{3}{4}$ to $I \frac{1}{2}$ in. in diameter. It is surrounded by an erythematous area of considerable extent, so that the whole reactive zone may be covered by the palm of the hand. After two or three days the reaction begins to disappear.

This test is even more delicate than that of ron Pirquet in revealing the presence of latent tuberculosis, and is at least as reliable for the detection of active foci. In accompanying advanced cachectic states and measles, and in meningitis and advanced stages of the disease, the reaction may, however, fail.

The chief interest of the test lies in the endeavours which have been made to utilise it as a measure of the reactive power of the tissues, and so to aid in prognosis and the determination of dosage. To this end, the skin over an area, say, of one or two centimetres is picked up by means of calipers and its thickness measured and compared with that of a similar area of skin at the corresponding site of the body where inoculation has not been performed. By repeating the test with dilutions of tuberculin weaker or stronger than that given above, a dilution may then be found which will induce an increase in thickness of the skin of any desired dimension. No definite results have as yet been obtained, but the procedure is well worthy of further investigation. 


\section{The Ophthalmo-Test of Calmette and Wolff-Eisner.}

For the performance of this test Calmette recommends a tuberculin prepared by precipitating bovine tuberculin with 95 per cent. alcohol, and freed by a complicated process from glycerine, resin, and wax, but still containing the potent bacterial bodies; the precipitate is then dissolved in distilled water to form solutions of $0.5,1,2$, and 4 per cent. strength. Bandelier and Roepke advise that the old tuberculin of Koch should be diluted with 0.85 per cent. sterile salt solution, or with 0.5 per cent. solution of carbolic acid in water, and state that this in I-4 per cent. dilutions of the tuberculin is unirritating to the healthy eye.

For a first test Calmette advises the $0^{\circ} 5$ dilution, and this is usually employed in this country; Bandelier and Roepke advise the $I \cdot O$ per cent. dilution.

The test is performed as follows: The patient sits on a chair, and throws the head slightly backwards. The physician draws down the lower lid, and lets one drop of the selected dilution fall gently from a drop-pipette into the lower conjunctival sac. The lid is kept retracted for half a minute, so that the conjunctiva is well bathed with the solution; the patient then looks upwards with the lids open for a similar period. The eye should not be bandaged, and the patient should be warned against subsequent rubbing of the eye.

The reaction may begin in four or five hours, but usually does so within twelve hours, and is at its height after an interval of twentyfour to thirty-six hours; occasionally response is delayed for two or three days. Three grades of reaction are distinguished:

(I) Slight-reddening of the caruncle and palpebral conjunctiva with some lachrymation.

(2) Moderate-intenser reddening with increased secretion, and reddening of the ocular conjuntiva; threads of fibrinous secretion may be seen in the conjunctival cul-de-sac.

(3) Severe-intense reddening of the whole conjunctiva, with chemosis, much fibrinous and purulent secretion, photophobia, and perhaps small ecchymoses.

In no case should there be rise of temperature or pain. The eye should resume its normal appearance in two or three days; occasionally this occupies five or six days, rarely ten to fifteen days.

In the event of a positive reaction not being obtained, some authorities advise the repetition of the test two days later upon the same eye with a tuberculin of double strength, and if this fail a further repetition two days later with a tuberculin again doubled in concentration. This procedure is, however, free from danger only when due 
regard is paid to certain contra-indications which are equally applicable to the first instillation; thus, according to Calmette, absolute contra-indication is given by any ocular disease, no matter the kind or stage, even if the processes have run their course. Unsoundness of one eye forbids the test being applied to the other. In children severe reactions are especially liable to occur, and in them the application of the test is best avoided.

So many serious results have now been recorded by careful and competent observers that the test has fallen into some disrepute, and is perhaps best left in the hands of specialists. Personally, I have never experienced any difficulty nor seen any ill-results come from it.

Wolff and Bandelier and Roepke report one peculiar difficulty which is liable to arise, namely, a recrudescence of the reaction after therapeutic inoculations with minimal doses of tuberculin-an effect which may be so constant and so persistent as to necessitate a suspension of the tuberculin treatment.

The diagnostic value of the test.- Repetition of the instillation has been opposed by various authorities on the ground that a previous instillation is able to set up a condition of hypersusceptibility in the tissues of a truly healthy individual. This is almost certainly wrong, and the frequency with which positive results have been reported in sufferers from typhoid and scarlet fever, syphilis, diabetes, etc., who were stated to be free from tubercle, is probably due to the fact that these individuals were only clinically free from tubercle, and in reality possessed latent or healed foci. A comparison of the results it yields with those given by the cutaneous test strongly confirms its true specificity.

Very cachectic cases and those suffering from very advanced phthisis may fail to give a positive reaction just as they will fail towards the cutaneous and subcutaneous tests. At the same time there is no doubt that it does not give a correct indication in a certain number of cases where it might be anticipated with considerable confidence, and it is unfortunately the case that one or even two instillations may especially fail to give correct guidance just in those cases where it is most needed, i.e. in the early stages of pulmonary tuberculosis. Furthermore, the fact that a case of perfectly quiescent phthisis may react strongly to a first instillation, while one with active mischief may require at least three, tends further to limit the practical utility of the test.

\section{The Subcutaneous Test and the Tuberculin Reaction.}

For the subcutaneous test Koch's old tuberculin is employed, and the procedure is as follows: The mouth temperature is taken every 
three hours, and if it prove to go over $37^{\circ} 2^{\circ} \mathrm{C}$., or $99^{\circ} \mathrm{F}$., the patient remains in bed until it has not risen above this level for at least two days. No attempt at reduction by means of drugs is permissible. The test should never be applied to females during or near the menstrual period.

The skin at the inferior angle of the scapula is sterilised and the inoculation performed aseptically. The appropriate initial dosage varies according to different authorities, but the following is perhaps the best: In weakly subjects and in suspected early cases in whom reactions are often marked, begin with ooor c.c.; with strong people begin with $0002-\cdot 0005$ c.c.; with children with $00005^{-000 I}$ c.c. If there is no reaction within tiventy-four hours, such as will be described, the procedure may be tabulated as follows:

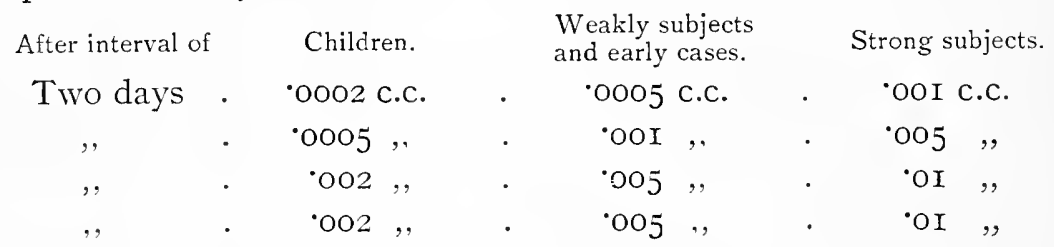

A definite reaction consists in a rise of temperature of $0.5^{\circ} \mathrm{C}$. or $I^{\circ} \mathrm{F}$, a doubtful reaction in a rise of $0.3^{\circ} \mathrm{C}$. or $0.5^{\circ} \mathrm{F}$. In the former case the experiment is concluded at that stage which gave the reaction. In the latter case the dosage is not increased, but is repeated on the day following that on which the temperature returns to normal; a definite reaction is then shown by a rise of temperature greater than before, a negative reaction by a smaller rise.

If a negative reaction is obtained with the fourth inoculation, that dosage should be repeated once in all strongly suspected cases to exclude definitely the diagnosis of tuberculosis.

Absolute contra-indications to the use of the subcutaneous test are-

(I) A rise of temperature to over $37^{\circ} 2^{\circ} \mathrm{C}$. which will not yield to rest.

(2) The presence of tubercle bacilli in the sputum.

(3) The fact that a definite diagnosis is already possible.

Other conditions which may contra-indicate the test are-

(I) Hæmoptysis; this, however, only necessitates waiting till all blood has disappeared for a week from the sputum, whereby full opportunity is afforded for the healing of the vessel, a possible reduction of the initial dosage and cautious increase of subsequent ones.

(2) Renal disease as evidenced by albumen, blood and casts in the urine. Only severe cases are excluded from the test, in others careful dosage is advisable. 
(3) Heart disease, but only if there be serere valyular disease or definite myocarditis.

(4) Epilepsy: As it has been noticed that epileptiform seizures which have been in abeyance for years may be again initiated, marked care is necessary if the clinical condition indicates the advisability of performing the test.

(5) Diabetes certainly necessitates care, and if the disease be advanced the question may well be raised, is it worth while?

(6) Hysteria: Here the result is apt to be upset by psychical rises of temperature; the cutaneous reaction must be substituted in this case.

(7) The suspected presence of miliary tuberculosis or adranced tuberculous ulceration of the intestines, especially if pain and tenderness be present.

(8) Age: In young children under the age of three the cutaneous reaction is quite reliable and is to be preferred; in older children the subcutaneous test is quite safe and should be employed.

Although the rise of temperature is the main point upon which most diagnosticians concentrate their attention, it is only one of the resultant phenomena which may be encountered. The complete tuberculin reaction consists in (I) local, (2) general, (3) focal disturbances.

(I) The local reaction may be of any grade, from complete absence to the production of a painful red swelling with cedema of the subcutaneous tissues for an area of $\mathrm{I}^{-2} 2$ in. in diameter around the puncture. and of pain and redness along the needle track due to the escape of a small quantity of the tuberculin when introducing or withdrawing the needle.

The local reaction usually subsides in two or three days.

(2) The general reaction is due to toxic effects upon the central nervous system. Characteristically, as we have seen, the most important feature is a rise of temperature of at least $0^{\prime} 5^{\circ} \mathrm{C}$. or I ${ }^{\circ} \mathrm{F}$. A rise to $38^{\circ} \mathrm{C}$. is designated "slight," one to $39^{\circ} \mathrm{C}$. "moderate," and one over $39^{\circ}$ C. " severe." Most typically it consists in a rise of temperature which begins within six to eight hours, reaches a maximum in twelve to sixteen hours, and subsides to normal in twenty-four to fortyeight hours; in time of onset, height and duration of rise great divergences from this normal course may be encountered, so that it is hardly possible to speak of a typical curve. Sometimes the rise of temperature begins within four hours, sometimes it is delayed for thirty hours, sometimes the fall of temperature is by crisis, more often perhaps it is by lysis; secondary oscillations may be seen.

In mild forms the rise of temperature and a siight acceleration of pulseand respiration-rate and a slight malaise constitute the whole of the general reaction; more often there is some pain or ache at the site of 
infection, some headache of varying degree, and more pronounced malaise and acceleration of pulse and respiration ; occasionally there is very marked malaise, nausea and yomiting, photophobia, rigors, pains in the limbs, thirst and sleeplessness; loss of weight even up to one pound may also result. Such severe forms of the reaction as this are especially liable to occur with very slight and early degrees of infection. Personally I have only met with them in such cases and with the initial dosage of only ooor c.c. These unpleasant disturbances as a rule subside with great rapidity and no ill-effects whatever remain after the lapse of twenty-four hours; should they persist there is no objection whatever to the administration of 5 to Io gr. of phenacetin or antipyrin, 8 gr. of pyramidon, or Io gr. of bromural.

When the reaction finally passes off it generally leaves behind it a decidedly increased feeling of general well-being and comfort; in any case no harm ever results in these days of low initial dosage and gradual increase.

(3) The focal reaction: Whether a focal reaction, i.e. one occurring at the site of the lesion, often occurs in the absence of a preceding local and general reaction is a disputed point. That it does sometimes occur cannot be questioned; some observers, like Hamman and Wolman, say they have never detected an increase in the physical signs in the lungs without some evidence of a general reaction. Carl Spengler has observed it preceding the general reaction, Turban has noticed its occurrence in the absence of the general reaction, and I myself have never failed to detect a distinct alteration in physical signs, preceding, accompanying or succeeding any general reaction, even of the smallest degree. Of all the signs of response to inoculation with tuberculin it is, pace many observers, the most sure, the most definite and the most easily obtainable with minimal doses of tuberculin, the essentials for its observation being careful charting of the physical signs before inoculation and at intervals of four hours thereafter, beginning eight hours after the inoculation and being continued if necessary till the lapse of another forty hours. The increase of physical signs and symptoms is due to exudation set up by the induced hyperæmia, and is often accompanied by slight pain or ache of the affected parts and by increased expectoration.

Personally I believe that the production of a focal reaction is an absolute essential for the production of a general one. Were the focal reaction not an inevitable result of the administration of diagnostic doses of tuberculin how utterly futile would be the subsequent treatment of the patient with the much smaller therapeutic doses, for on the hyperæmic and other changes induced at the foci of disease does the curative action of the tuberculin mainly depend. 
This leads us to the consideration of the theories advanced in explanation of the tuberculin reaction. All of these it would be little profit to discuss, but two or three require mention.

Let us first see what are the main facts that need explanation. They are these: that doses of even $\frac{1}{10}$ c.c. of old tuberculin can be administered to healthy men and I c.c. to healthy guinea-pigs without producing any general disturbance whatever, whereas the administration of $\frac{1}{1000}$ c.c. will, in the case of many tuberculous human beings, cause a marked general reaction and often kill an infected guinea-pig. Healthy individuals are thus very tolerant to the toxin contained in tuberculin, while infected ones are very intolerant.

One suggestion put forward to explain these points was that the difference was due to the fact that the injected individual already contained a considerable amount of tuberculo-toxin, and that a reaction was due to the summation with this of the injected toxin. This mere surnmation theory is quite inadequate to explain all the facts.

Wassermann and Bruch have advanced another theory based on the observation that in the serum of patients treated with tuberculin, and at the infected foci of untreated patients, there is a substance which, with tuberculin, causes absorption of complement, and to this body they have given the somewhat unfortunate name of "anti-tuberculin" as if it were an antitoxic substance neutralising tuberculin, 'instead of being a body which activates tuberculin.

They therefore suppose that reactions occur when tuberculin meets with anti-tuberculin in the foci and fixation of complement results.

Objections to this theory are many; thus it is difficult to see how both tuberculin and anti-tuberculin can exist together in tubercular foci ewithout combining with each other; secondly, it is hard to understand how a combination of tuberculin and anti-tuberculin in which chemical affinities are satisfied can cause inflammatory reactions; thirdly, it is incomprehensible that the combination can have a harmful action on the foci and at the same time a detoxifying action in the blood.

The third and most generally accepted theory is that of WolffEisner. He bases his theory on the fact that all foreign albumins introduced into the tissues are dealt with by the production of specitic antibodies-such as lysins and agglutinins. He therefore assumes that tuberculin is an albumin of low toxicity, and requires breaking down into bodies of less complex constitution, one at least of which is highly toxic, before any toxic action is noticeable.

Sensitive tissues, i.e. those of an infected individual, differ from insensitive tissues, i.e. those of a healthy individual, in that the former contain this tuberculo-lysin and the latter do not. As an important adjunct to this thecry, Wolff-Eisner considers that the cells of the 
infected individual have acquired a hypersensitiveness to the foreign albumin of the tubercle bacilli, comparable to the hypersensitiveness to tetano-toxin evidenced by horses which have been immunised against tetanus.

This theory is the simplest and most satisfactory yet advanced, but Wolff-Eisner has not yet himself adequately explained the production of focal reactions. The theory may, however, be modified and extended as follows, and made to explain similar phenomena encountered in the therapeutic use of all vaccines.

Tubercle bacilli and tuberculin and other bacteria in such doses as are administered are practically devoid of toxicity; they excite the production of the corresponding lysin, which breaks them down and forms a toxic decomposition product. This lysin we know is formed rapidly and in an amount far in excess of that required to lysinise the few bacteria contained in the inoculum; it is rapidly carried all over the body and to the infected foci, where it lysinises the living infective agents and liberates fresh toxin. The toxin formed from the inoculum may suffice to produce a local reaction, but it is insufficient to produce focal and general disturbances, as must surely be admitted when one considers the minuteness of the total albumin content of a therapeutic dose; the toxin liberated at the infected foci may, however, suffice to cause both focal and general reactions. A focal reaction of some degree, however slight, is inevitable; a general reaction will be produced only if the amount of toxin be sufficient, and the activity with which the body responds to it by forming antitoxin be not great. It is thus obvious that the focal reaction, though certainly present, may escape detection, because the induced effects of the toxin may be too slight or ill-adapted to detection by means of the stethoscope, and that, on the other hand, a general reaction may be too slight for observation, owing either to the small amount of toxin carried into the circulation and so to the nerve centres from the infected foci, or from its rapid neutralisation by antitoxin formed by the tissues in response to the elaboration of toxin.

The stimuli brought to bear on the tissue-cells by the toxin may be slight and exercise a stimulating effect, so that the cells respond with extra readiness to fresh toxin, i.e. they may be rendered hypersensitive, or they may be of such magnitude that they exercise a poisoning, or rather, paralysing effect, and so induce tolerance to the poison.

This theory, as I have elaborated it, is simple, in accord with physiological and bio-chemical facts, and seems to offer a full and reasonable explanation of all points of difficulty. 


\section{The Diagnostic Value of the Subcutaneous Test.}

The subcutaneous test is especially to be employed when it is necessary either to make an early diagnosis in doubtful cases, or to decide the course of treatment in cases where there is difficulty in arriving at a differential diagnosis. If the test be conducted according to the lines I have laid down and careful watch be kept for a local as well as for a general reaction it is devoid of all danger; a little extra care will enable the general practitioner to utilise it with a freedom equal to that applicable to the case of the specialist. As we have seen, the cutaneous, percutaneous and conjunctival tests give indication of anatomical as well as of clinical tuberculosis, and their practical utility is greatly limited thereby. If by means of the subcutaneous test a focal reaction is produced, ample evidence is afforded of the presence of active, or, at least, of incompletely healed tuberculosis. That mild, general reactions may be evidenced in cases of latent and of healed or anatomical tuberculosis is true, but the percentage of cases in which this occurs is decidedly less than with the tests already described.

A positive reaction, focal or general, sometimes fails to appear in slight inactive cases where the disease has become stationary, and also in very advanced and cachectic cases.

In briefly summarising the value of specific products in the diagnosis of tuberculosis, I cannot do better than quote the opinions of Bandelier and Roepke, with whom I am in complete accord: "In the early diagnosis of pulmonary tuberculosis no crucial significance can be admitted for the local reaction methods. On the other hand, no objection can be raised to the employment of them, singly or in combination, before the subcutaneous test, on account of their simplicity and the obvious advantages they possess in the presence of fever and other troublesome general symptoms. But their disadrantages must not be lost sight of, especially their uncertainty. If both cutaneous and conjunctival tests are positive, then tuberculosis may be decided upon without any indication of its site or character. Is one or both negative, the absence of a tubercular focus is not demonstrated; on the contrary, it must again be emphasised that both methods may fail in spite of active pulmonary tuberculosis being present. In all these cases the subcutaneous method has finally to decide whether tubercular disease is present or not. It is still par excellence the diagnostic agent for the detection of early pulmonary tuberculosis in adults. Its value is increased by the fact that in a high percentage of subcutaneous tests focal reactions make their appearance and allow a conclusion as to the site of the tuberculosis in the lung." And again: "The value of tuber- 
culin in diagnosis is well established. The individual methods have their special advantages and disadvantages. For each particular case, to make use of the advantages and exclude the disadvantages is the task of an individualising diagnostic method. In diagnostic certitude the subcutaneous test stands first; for prognosis all methods are equally futile. Tuberculin diagnosis may be introduced into general practice without impairing the results. Ill-effects to the patient may with certainty be avoided by proper selection of cases and conscientious attention to details. Tuberculin, by making early diagnosis possible, is destined to be of service in the efficient prophylaxis and treatment of tuberculosis."

\section{Opsonic Inidex Estimations in Diagnosis.}

In the performance of this test the introduction of the specific products may be from without, or it may be from within, the inoculations then occurring at the infected foci either from spontaneous or induced auto-inoculations. This test, which once enjoyed a considerable vogue in England, fell into desuetude and neglect, owing partly to lack of care on the part of many in performing the estimation, partly to ignorance of all the possible causes of fallacy. It is now being revived, and promises to occupy a position of deserved esteem. For an account of the many necessary precautions which must be taken in order to assure a reliable result, the reader is referred to a paper by Colebrook on "The Opsonic Method in Relation to Tuberculosis" (Practitioner, January, I9I3, p. I38). The test depends upon these observations: (I) That the opsonic index of the serum of the healthy individual towards the tubercle bacillus lies between the limits 0.8 and $x^{\cdot} 2$, and usually between the limits $0^{\circ} 9$ and $I^{\cdot} I$; (2) that the opsonic index of infected individuals tends to vary from day to day, so that on occasions it may diverge considerably from these limits; (3) that the opsonic index of a healthy individual is little disturbed by the administration of doses of tuberculin below ooor c.c.; (4) that the opsonic index of an infected person will be caused to change in a perfectly definite manner by a similar administration

In its simplest form the test is conducted by estimating the index of the individual upon several different occasions when he is pursuing his normal mode of living.

In a more definite form the test is applied by first keeping the patient at rest, and then making him auto-inoculate himself with his own tuberculin either by the performance of deep breathing, climbing upstairs or up a hill, or by some other form of active exercise. Specimens of blood are taken just before the exercise is begun, 
and at intervals of I, 3, 6, I2, and 24 hours thereafter and the indices estimated. If considerable fluctuations are observed autoinoculation with tuberculin derived from his own bacilli is indicated, and active tuberculosis is almost certainly proved to be present.

If no signs of auto-inoculating processes are thereby indicated a dose of 'ooor c.c. of old tuberculin or of T.R. may be given. Numerous experiments have shown that the effect of this upon a healthy individual is to produce a lowering of the index perhaps for a day of not more than a decimal point, and that this, is succeeded by a short corresponding elevation. In the infected individual the effect is much more marked; a depression of two or three decimal points may result, and not pass off for one or several days, and is succeeded by a marked elevation, which falls only gradually and slowly.

Provided that the various observations are carried out by an expert in opsonic index estimations, who is in constant practice and is conversant with all the various possible fallacies and disturbing influences, an indication is obtained which is of the very greatest service as a diagnostic aid. In delicacy and accuracy the method often excels all others, the subcutaneous test included, and it has the cardinal advantage of revealing the presence of active tuberculosis only. The great drawbacks lie in the limited number of dependable experts in index observations available and in the considerable expense which must necessarily be incurred. Not only is it capable of affording great help in the early diagnosis of tuberculosis, but also of enabling the determination to be made with considerable accuracy as to when all active processes of disease are in abeyance.

\section{Classification of Cases of Pulmonary Tuberculosis.}

From the point of view of tuberculin therapy the classification of cases of pulmonary tuberculosis according to the extent and distribution of the lesions, as in Turban's classification, is of no particular significance. Of much greater importance is the nature of the processes at the fociof disease and the manner in which the body is making response to the infection. For instance, the prognosis in even a very early case of miliary tuberculosis is much less favourable than that of an acute pneumonic tuberculosis of a single lobe, and that of the latter less favourable than that of a fibro-caseous involvement of more than one lobe.

Tuberculin is not directly a curative agent in the sense that antidiphtheritic serum is, it is merely an active immunising agent, a stimulant for the tissue-cells of the formation of specific antibodies. It therefore follows that the tissue-cells must be in a condition which enables them to respond to the stimuli and elaborate the neces- 
sary antibodies. The period of disease at which the body can best perform this task is, as Koch so strongly urged, in the early stages of a strictly localised infection. In the earliest stages of miliary tuberculosis Koch detected a definite attempt on the part of the body to elaborate specific antibodies, but this power is soon lost and the cells can no longer respond to stimulation, as is clearly shown by their failure to react to the various diagnostic tests. For a similar reason in cases of advanced cachexia tuberculin is of no avail.

Many authorities would restrict the use of tuberculin to uncomplicated afebrile cases in Stages I and II of Turban. i.e. to cases whose temperature does not rise above $99^{\circ} \mathrm{F}$., in which cavitation has not yet occurred, and where the process is confined to not more than two lobes. Others would exclude all cases in which mixed infection is present, but fail to realise that mixed infection is almost certainly present in all cases of open tuberculosis, and inasmuch as they administer the drug to many such cases obviously fail to observe their own dictum. Mixed infection, per se, is no contra-indication to the use of tuberculin, but, as we have seen in Chapter $\mathrm{X}$, so complicates the control of dosage and intervals by obscuring the focal and general reaction thereto that the difficulty of administration is immensely increased.

Actively progressive forms of the disease are not likely to be benefited, but advanced forms where much destruction has occurred may recover, provided the destruction falls somewhat short of that degree in which pathological anatomy shows that spontaneous cure or arrest may take place. The best method of classification is that based upon a consideration of the nature of the processes which are occurring at the foci of disease and of the manner in which the body is responding to the infection; such an one is that proposed by Inman, who divides cases into the following three classes:

Class I : Resting febrile, i.e. those which show a temperature of $99^{\circ} \mathrm{F}$. or over when at rest in bed.

Class II : Ambulant febrile, resting afebrile, i.e. those which remain afebrile so long as they are at absolute rest, but become febrile when allowed to get up and take exercise. Class II is often a transition between Classes I and III.

Class III: Ambulant afebrile, i.e. those without fever in spite of exercise or work.

The febrility of tuberculosis is, as Inman and Paterson have shown, due to auto-inoculations with specific products from the bacilli at the foci of disease, but it is necessary to take a somewhat wider view of what constitutes the auto-inoculations than is done by these observers. They appear to regard them as being composed of the products of the tubercle bacilli alone, whereas in many cases they also consist 
of the products of the bacilli which constitute the mixed infection. Repeatedly one sees a case of "ambulant afebrile" tuberculosis suddenly become "ambulant febrile," and probably eren "resting febrile." I have carefully examined sereral such cases, and have in every instance found that the factor at work was a fresh invasion by other than the tubercle bacilli. I do not mean to convey that this always is the case, and that exacerbation of the tuberculous infection is never responsible for the change of type, for of course it is. As a rule, however, the following distinction holds: In "ambulant afebrile " cases which suddenly become "resting febrile," the factor usually is the access of mixed infection, whereas, when they become "ambulant febrile," but "resting afebrile," the factor usually is exacerbation of the tuberculous process.

The researches of Inman and Paterson have shown that in the resting febrile cases of Class I the stimuli are excessive and irregular, and tend to orertax the mechanism of immunity, and that unless these stimuli can be controlled by absolute or "typhoid" rest the course is ineritably downhill, whereas in the ambulant febrile but resting afebrile cases of Class II the stimuli are adequate and controllable, and such cases tend to get well; while, finally, in the ambulant afebrile cases of Class III the stimuli are insufincient and the tendency is torrards little activity and chronicity. By absolute or "typhoid" rest they have shown that cases of Class I may sometimes have their stimuli reduced and controlled, and so they may be brought into Class II or III.

Cases of Class II may become cured if the stimuli be adequately maintained for a sufficiently long time, but they tend to gravitate into Class III, or into Class I, if a suaden exacerbation of the tuberculous process occur or a mixed infection be superadded.

Class III comprises the chronic cases with long periods of latency and inactivity, alternating with occasional relapses.

These considerations considerably facilitate the selection of cases suitable for tuberculin treatment, and also indicate the proper time for its inception. Thus all ambulant afebrile cases are suitable for the administration of tuberculin; many will perhaps get well without specific medication, but experience tends to show clearly that tuberculin properly administered will markedly increase the ratios of recoveries and will do no apparent harm in any instance.

In the ambulant febrile and resting afebrile cases the immunising impulses can be controlled by carefully graduated rest and exercise, and if this be done and the best hygienic conditions be maintained the tendency is towards recovery. A necessary sequel to this is that sooner or later such cases must pass into the category of the "ambulant afebrile," where, as we have seen, the stimuli tend to become 
inadequate. On the other hand, a certain percentage of the cases of Class II tend to gravitate into Class I, and so towards a fatal issue.

It therefore follows that inasmuch as tuberculin induces a curative effect, its application in cases of Class II will tend to expedite the recovery of such cases as gravitate into Class III, and will diminish the proportion of cases which tend to gravitate into Class I. Practical experience shows that these anticipations are fulfilled. Cases of early tuberculosis almost alvays fall into this category, and were considered by Koch to be those to whom tuberculin was especially likely to prove beneficial. But, it may well be urged, why interfere with cases that are obviously improving? This is a perfectly valid objection, but experience has shown that tuberculin properly administered is devoid of harm, and may be productive of good even in the most ripidly improving cases. Its administration also has the advantage that it necessitates the patient being kept under close observation, and so facilitates the early detection of any unfavourable tendency. If the preference, however, is for the treatment of these cases on non-specific lines, it then becomes incumbent to maintain an especially close watch upon the patient, with a view, on the one hand, to increasing the immunising impulses when they tend to weaken, and, on the other hand, to controlling the impulses when they tend to become excessive and irregular.

It remains now to consider whether tuberculin treatment is applicable to any cases belonging to Class $\mathrm{I}$. As we have already seen, the temperature of all three classes is due either in whole or part to the action of tuberculo-toxin reaching the central nervous system from the foci of disease; in cases of Class I it is especially important to ascertain whether the pyrexia is due, entirely or only in part, to this influence. The other possibility is the presence of mixed infection, which is also by no means excluded as a factor in cases belonging to Classes II and III. This question of mixed infection has been fully dealt with in Chapter $\mathrm{X}$, where full particulars have been given for its detection and treatment. The prime consideration, therefore, in the treatment of cases belonging to Class $I$ is the determination as to whether the pyrexia is due to the tubercular infection alone or to mixed infection as well. If mixed infection be an important factor, treatment with the appropriate vaccines will probably settle this question and assist in bringing these cases into the category of Class II, when tuberculin treatment may be considered.

If the influence of mixed infection is disproved, the question has to be considered whether tuberculin will afford material help. Theoretically it would appear unlikely that the administration of further stimuli could assist in the control of excessive and ill-ordered stimuli, 
and clinical experience tends to confirm this view. In a small percentage of cases it is, however, found that minimal doses of tuberculin do assist in the raising of toxin immunity. If absolute or "typhoid" rest fail to influence the pyrexia of these cases, the issue is practically certain to be a fatal one, and it is therefore justifiable, after explaining the state of affairs to the patient, to make a last endeavour to influence the course of the disease for good. Cure may be a very remote prospect, but increase of comfort may be brought about and the ultimate end may be postponed. Any active interference must, however, be conducted with extreme care, or the effect may be the reverse of that intended.

Finally; a few words must be said as regards the question of when to begin tuberculin treatment in cases which are suitable for this procedure. The usual answer given is - "As soon as possible." Personally I think this needs some qualification. Cases with mixed infection are not necessarily to be excluded from treatment, and, indeed, most observers frequently treat such cases with tuberculin, failing to recognise that mixed infection is really present; in these it is not wise to combine vaccine and tuberculin treatment, for the effect of each is likely to mask the effect of the other. I think I have amply demonstrated in Chapter $\mathrm{X}$ that the wiser course is to deal first with the mixed infection and then with the tuberculous process, so that I think the proper answer to the question is "as soon as possible after any mixed infection present has been adequately dealt with."

\section{The Choice of Tuberculin.}

As a necessary preliminary to the consideration of this question it is well to recapitulate a few important points.

Firstly, that tuberculin is an actively immunising substance; it contains albumins constituting the toxic elements formed by the bacterial growth, as well as those constituting the essential protoplasmic substance of the bacilli.

Some of the tuberculins contain only one or other of these bodies, some contain both.

The human tissues are capable of elaborating antibodies to these albumins, so that immunity can be excited both against the toxins and the bacilli themselves. Of these antibodies little is known, but among them lysin, agglutinin, opsonin, antituberculin and possibly antitoxin may be mentioned.

In cases of pulmonary tuberculosis the general symptoms are due to the action of a toxic component of lysinised tuberculin upon the central nervous system, the focal changes are due chiefly to secreted 
endotoxins, the range of their activities being more or less confined to the infected areas. The true ultimate objective of treatment is to bring about extinction of the infection, but the production of bactericidal effects is complicated by the existence of a peculiar sensitiveness of the tissues to lysinised tuberculin, and the consequent necessity of establishing a high degree of tolerance to the toxin. When general symptoms are little marked these difficulties are minimal, but when constitutional disturbances are severe or easily excited it is necessary that attention should be directed to the establishment of a high degree of tolerance.

The statement is often made that all the varieties of tuberculin are capable of producing the same results, the only difference being that some are more powerful agents than others. But with this I do not absolutely agree: there is, and must be, a difference in the immune bodies which they are capable of inciting corresponding to the difference of their constitution. Thus T.R., and to a less degree B.E., will lead more especially to the production of antibodies to the protoplasmic albumin; T.O.A., and old tuberculin, on the other hand, will incite the production of antibodies more especially to the soluble toxins, and so will the more powerfully stimulate the development of tolerance.

While old tuberculin is absorbed with considerable rapidity, B.E. is absorbed very slowly; the production of antibodies is correspondingly affected, and it therefore follows that in febrile and highly sensitive cases B.E. is less likely to lead to violent reactions than is old tuberculin, and this preparation is therefore preferred for use at the beginning of the treatment of such cases.

Sahli and others state that of all tuberculins Beraneck's contains the least amount of non-specific toxins, and at the same time a high content of specific toxin and essential protoplasmic albumin; they therefore consider it to be the best preparation for general use.

The preparations derived from the bovine strain of bacilli probably differ from those derived from the human strain mainly in the direction of being much milder in their actions, and therefore less liable to produce severe disturbances.

Although it is not possible to give definite values for the immunising powers of the various tuberculins, it is possible to arrange them roughly as follows in descending order, the attached numerals being an approximate value of their respective strengths: Denys' B.F. (500), T. (200), P.T. (40), B.E. and T.R. (5), and P.T.O. (I).

Until one has had a fair amount of experience of tuberculin therapy, it is well to restrict oneself to the use of a few preparations only; for most purposes old tuberculin, or T., will serve well, and it is perhaps advisable to employ this preparation alone till considerable practice has 
brought familiarity with dosage, and the various ways in which the human tissues react thereto. In dispensary work and the treatment of ambulant cases the use of P.T.O., P.T., and T. in sequence has much to recommend it, the slight toxicity of the first preparation minimising the risk of exciting undesirable reactions.

Personally, I think that in every instance treatment should be concluded by a course of bacillary emulsion, a two-fold reason for this existing in the facts--(I) that this preparation is a whole bacillary product. and is therefore best suited for the production of anti-bacterial immunity, and (2) its rate of absorption being slow the immunity which has been established is thereby maintained at a high level for a considerable period. As transition is made from one tuberculin to another. it is necessary to keep in mind the approximate relative strengths of the respective preparations; for instance, if the dosage of P.T.O. has been raised to O'I c.c., and it is thought advisable to make substitution of P.T.. it will not be good practice to begin with a minimal dose such as for tolerance to drop from that high level to which it has been raised with so much care. The dose of P.T. corresponding to $0^{\circ}$ I c.c. of P.T.O. being about $\frac{n \cdot 1}{40}$ c.c. or 0025 c.c., there will be little danger in beginning with a dose of 0005 c.c. of P.T. A similar calculation will give the appropriate initial dose of other preparations as they are substituted in turn. Personally, in making transition, I prefer to begin with only one-fifth of the calculated dosages.

\section{Conduct of the Course of Treatment: Dosage and Interials.}

The procedures which have been devised for the administration of tuberculin are almost as many in number as are the specialists in the use of the drug. It is quite impossible to enter into a discussion of the merits and demerits of each, and I propose to give only such general instructions as experience has shown to be applicable to the great majority of cases, and to point out how these may require modification to suit individual cases, for let me once more emphasise the fact that in tuberculosis, more than in any other disease, it is necessary to pay the most strict attention to the peculiarities of each individual case, and that it is only by doing this that the incidental dangers can be avoided and the best results secured.

While it must be admitted that the establishment of a high degree of tolerance of the tissues to tuberculo-toxin is a very important essential, inasmuch as it is only by the achievement of this end that the grave constitutional disturbances due to intolerance of the toxin can be remored and kept in abeyance, I would join serious issue with all those who make this the sole objective of their treatment, and assume 
that high tolerance and extinction of infection go hand in hand, and are necessarily the same thing. The true aim of all treatment should be the extinction of the infection, and the best indication of progress in this direction is the condition of the local lesions. Now, observations of pulse and temperature and the general condition of the patient are easy, and are carried out as a mere routine in the treatment of all severe forms of bacterial infection, but however valuable they may be in themselves they do not necessarily afford a good indication of the condition of the local lesions, and this contention holds with especial force in regard to pulmonary tuberculosis. No sound observer would be content to treat a case of tuberculous laryngitis without making close scrutiny of the progress of the lesion, nor be satisfied that all was going well with a case of pneumonia without carefully examining the chest, yet many, if not most, authorities advise the control of tuberculin therapy by means of pulse, temperature, and general condition, to the neglect of careful observation of the chest. For this two things must be held responsible: firstly, disinclination or inability to devote the time to careful systematic stethoscopic observations; secondly, the entirely fallacious view already mentioned as held by many, that a general reaction can be elicited in the absence of a focal one. I. have already mentioned the production of the former is dependent upon the production of the latter, for the amount of toxin that can be formed by the tuberculo-lysin from any but the largest therapeutic doses of tuberculin falls far below that necessary for the poisoning of the central nervous system upon which constitutional symptoms depend; the local reaction may be so produced, but the focal and general reactions are dependent upon the increased elaboration of antibodies set up by the inoculation, and the action of these upon the innumerable bacilli at the foci of infection. It is a matter of common observation that marked focal changes can be detected in cases of lupus, tuberculous laryngitis and iritis after such a dose of tuberculin as fails to produce the least constitutional disturbances, and it is only the lack of careful stethoscopic observations at suitable intervals that has given rise to this prevalent fallacy. Occasionally, of course, lesions may be deep-seated in the lung, or be productive of few signs discoverable by the stethoscope, but a considerable experience enables me to affirm that such cases are clinically rare; I have never yet seen a case in which alterations, at all events in breath-sounds, were not produced by a therapeutic dose of tuberculin which sufficed to produce even the mildest constitutional disturbance. Such cases may, however, exist, and there are others where intolerance is very marked relatively to the focal reactions, and it is only in these, and in cases where opportunities cannot be afforded for close clinical observations, that the guidance 
of the general reaction should be substituted for that of the focal reaction.

The best procedure of all obviously is that wherein due regard is paid to all the varying aspects of the case, and wherein the local, focal, and general reactions are all carefully correlated and weighed together.

The procedure to be followed in the stethoscopy of the chest after the administration of a therapeutic dose of tuberculin is precisely similar to that which has already been so fully described and illustrated in Chapter $\mathrm{X}$, pp. I55-I70, for the control of dosage and intervals in the treatment, by means of vaccines, of the mixed infection, and needs little further consideration.

As with all other methods, treatment is begun with such a minimal dose of the selected tuberculin as the clinical condition of the patient and other considerations indicate; this, for most cases, may be oooor c.c. of either P.T.O., T.R. or B.E, '000005 P.T., •000002 T., '00000I T. Beraneck; if the case be a pyrexial or very early one half the above dosages may be employed. If the patient be a strong healthy subject or the disease be of long standing and few symptoms, the above dosages may be safely increased tivo- or even five-fold, but inasmuch as the increase of dosage is, as a rule, rapid, there is little occasion for this. If neither focal nor general reaction results, the initial dosage is doubled in three days, and this procedure continued until one or other reaction is produced, a focal one being, as $I$ have said, almost always discoverable before a general one. As soon as a dosage is attained which does produce a reaction a slight reconsideration of the clinical features of the case is necessary, the principles of tuberculin therapy differing slightly from those of other forms of vaccine therapy in that tolerance has to be taken into account as well as antibacterial immunity. Accordingly, if tolerance is low, as evidenced by slight degrees of exercise causing auto-inoculatory phenomena, it is desirable to produce a rapid increase of tolerance; clinical experience has shown that this can only be done by giving increased dosages at short intervals, viz. of about three to five days. Under these circumstances increased dosage will be given at each inoculation provided that careful observation of the focal reactions show that these are not being unduly excited, and that sufficient time is allowed to lapse between inoculations to permit the local condition deriving full benefit from the focal reaction; the fulfilment of this condition usually necessitates a slightly longer period being allowed between inoculations than would be allowed if the raising of tolerance were the sole objective-in other words, the intervals may have to be five to seven days instead of three to five days. On the other hand, if tolerance is adequate, as shown by the absence of auto-inoculatory phenomena, the necessity for rapid increase of 
dosage does not exist, and so long as a certain dosage produces a satisfactory focal reaction there is no need to go beyond it. As a rule it will be found that after three or four doses of a given magnitude focal reaction begins to fail : this is the signal for immediate increase of dosage. A very satisfactory scale for dosage is that employed by Bandelier and Roepke, viz. I, I·5, 2, 3, 5, 7, IO, I5, 20 and so on ; this will serve for the great majority of cases, but will need modifying to meet individual peculiarities.

It should be remembered that if at any time increased dosage produces an excessive reaction or undesirable constitutional effects in any way, prolonged rise of temperature or of pulse-rate and loss of weight being the most important symptoms under this category, return should at once be made to that dosage which failed to give rise to these illresults, and any subsequent increase is to be made with especial care and caution.

The temperatures which should be taken for control purposes are best determined experimentally for every case, for some have their highest temperatures at one hour of the day, others at a totally different hour. Before treatment is ever begun it is therefore wise to send the patient to bed, in order that rectal temperatures may be taken every three or even every two hours, and that careful clinical study may be made of the patient as an individual. Thereafter it will, as a rule, suffice to take the temperature at such times as correspond to the usual daily maximum and minimum, and if there is any great objection to rectal temperatures, mouth temperatures, or, better, those taken in the stream of urine, may be substituted. During the twenty-four hours following an inoculation three-hourly temperatures are, however, most desirable.

Under this scheme of treatment it will be seen that primarily attention is focussed upon the changes induced at the foci of infection, secondarily upon the general reaction and constitutional effects, and thirdly upon all the signs and symptoms that can give any indication of the progress of immunisation, such as the sputum, urine, appetite, body-weight and feeling of general well-being.

The near completion of a course of treatment is indicated by :

(I) The complete disappearance of all signs and symptoms;

(2) A failure to incite auto-inoculatory phenomena by hard exercise or work; and perhaps-

(3) the failure of such dosages as $0^{\circ}$ I to $0^{\circ} 2$ c.c. of old tuberculin, tuberculin Béraneck, or Denys' bouillon filtré to produce either a focal or a general reaction.

After this stage has been reached it is well to continue treatment with a few further doses of the patient's optimal maximum strength, i.e., of that dosage which produces the best clinical results, given at 
lengthening intervals such as one week, two weeks, three weeks, and finally one month.

No matter how complete a cure may appear to be it is highly desirable that the patient should return every four to six months for careful re-examination of the chest and general condition. The physician should then also determine whether auto-inoculation can be induced, and also estimate the degree of tolerance by means of cutaneous tests with such dilutions of tuberculin as 5 per cent., Io per cent., 25 per cent., $5^{\circ}$ per cent., and Ioo per cent. Should any suspicion of relapse exist a fresh course of immunisation is to be strongly urged upon the patient.

Difficulties in the course of treatment are almost sure to arise, but the number and magnitude of these may be reduced by the proper selection of cases. They arise at any time-at the beginning, near the end, or in the middle of treatment.

When they occur at the beginning they are, as a rule, due to the high reactivity of the tissues to tuberculin, i.e. to a high degree of sensitiveness, and are especially liable to be encountered in very early cases and in those that have reacted strongly to a diagnostic dose of tuberculin. In one instance, a very early case indeed reacted to a diagnostic dose of $\cdot 000$ I c.c. of old tuberculin in a very violent manner. After two months' waiting I endeavoured to begin tuberculin treatment, but doses even of 'Ooooo I c.c. of P.T. and P.T.O. induced such marked constitutional upsets, including loss of weight of $\frac{1}{2}-\mathrm{I} \mathrm{lb}$. per week, that it was thought almost hopeless to continue specific therapy in the face of such extreme sensitiveness. Difficulties in the middle of treatment are more likely to arise from the onset of mixed infection or from a revival or re-exacerbation of a mixed infection which prior vaccine treatment had failed to eradicate entirely than from any other cause. Sometimes it will originate from the non-correspondence of the optimal therapeutic dosage with that which the patient can then tolerate; his tissues may be able to tolerate far larger doses than they are actually receiving; general reactions may be therefore conspicuous by their absence, focal reactions may be very difficult of detection, and accordingly the limits of dosage best suited to the needs of the patient are exceeded, little or no indication being given. for some time that such is the case. The clue will usually be afforded by a process of exclusion, and by such symptoms as slight loss of appetite and weight, depression and feeling of not being so well taking the place of previously perfectly satisfactory ones, and this in the absence of any unfavourable signs at the foci of infection. The suspension of the treatment for a couple of weeks and resumption then with dosages reduced to one third or one fourth will do little harm whenever suspicion exists of such a state of things, and may be productive of much good. 
The difficulties which may arise towards the end of treatment are concerned chiefly with the determination of ultimate dosage and the time for suspending further treatment. It is a mistake to fix any maximum or minimum limits to the final dosage. Some authorities endeavour always to reach I c.c. of old tuberculin; others are content with a tenth or less of this amount. In all cases where auto-inoculatory symptoms have been marked and where tolerance has shown a tendency to rapid fall it is very desirable to establish a high degree of tolerance by reaching a dosage of I c.c. of undiluted tuberculin if possible. In other cases the lower limit of o.I c.c. may be ample or it may even be unnecessarily high. This will be determined by the general features of the case and its behaviour during the course of specific treatment. Objection may be taken to my scheme for the conduct of treatment on the grounds that it requires too great individual attention, and occupies more time than can possibly be devoted to any case. My reply to this is that too great attention cannot possibly be given to the individual peculiarities of any case, and that a false conception may easily be formed of the time occupied in the examinations; while it is true that at the beginning of the course repeated examinations have to be made at short intervals, it is also true that once an accurate estimate has been formed of the reactive powers of the individual it is possible to predict with almost unerring accuracy the response that will be made to any dose; it therefore follows that just as clinical practice enables one to make a careful stethoscopic examination with greatly increased speed, so experience of the patient enables one to foretell the appropriate times for such examinations to be made.

I will now pass on to a brief description of the methods which I consider to be less scientific and less productive of clinical benefit than the foregoing, viz. those methods wherein the guidance utilised is that afforded by the general reaction or where little control upon dosage is made use of other than the general condition of the patient. In this connection three different schools exist: (I) That including Sahli, Denys and Wolff - Eisner, who aim at the avoidance of all general reactions. (2) That including Bandelier and Roepke and many workers in this country and America, who aim at avoiding all excessive reactions but at utilising mild ones. (3) That including Götsch, Möller, Lowenstein and Wilkinson, who pay little or no regard to strong reactions, and regard them as inevitable and as useful stepping-stones to the attainment of a very high degree of tolerance. The first of these schools hold that time is of little consequence in the treatment of a disease like pulmonary tuberculosis, which, as a rule, progresses so slowly. They are still more or less obsessed by the memory of the disasters of early days, and would avoid any possibility of producing a 
reaction which might have even the most temporary ill-effects, oblivious of the fact that improvement in some cases dates only from the time at which a general reaction of moderate degree was first excited, and that improvement in most of their cases begins with that dosage which just fails to produce a general reaction; in other words, with a dosage that in the great majority of cases produces that definite focal reaction which was not excited by some or many of the preceding smaller doses. Their view that focal reactions are not induced under their scheme of treatment is explicable only on the assumptions that their stethoscopic observations are not sufficiently complete or painstaking, not continued as the dosage rises, or not conducted at appropriate intervals after the administration of the tuberculin. In these particulars exception is to be taken to Sahli's methods, and many will not agree that the loss of time, whereby a course of treatment is drawn out to one, two or more years, is a negligible consideration; otherwise much is to be said for a practice wherein great regard is paid otherwise to individual peculiarities in reactivity and where in danger of ill-effects is reduced to a minimum. The plan of treatment is to begin with the smallest dose of one of the weakest solutions (e.g. ${ }^{\circ} 5$ c.c. of an 8192-fold or even greater dilution of Béraneck's tuberculin), which will always prove harmless, and then the dose is raised quite gradually in such a way that no manifest toxic actions (so-called reactions) ever occur, or if these are not to be avoided entirely they must be reduced to a minimum. As soon as any such phenomena occur, be they ever so insignificant, one must wait till they entirely disappear, and reduce the next dose to at least one half of that which last failed to produce any reaction; the interval between the doses must also be increased and the treatment subsequently conducted with greater precautions. Inoculations are given not oftener than twice a week with the concentrations up to the 32 -fold dilution; thereafter only once a week till the maximum dose is reached, when they are given only once a fortnight.

The dosage is gradually raised by $\frac{1}{10}$ or $\frac{1}{20}$ c.c. of each dilution till one reaches either the absolute maximum dose of I c.c. of undiluted tuberculin, or if the sensitiveness of the patient will not permit of this, till the individual maximum dose is attained. This must be found for each separate case; it will be one that can be tolerated without any ill-effects, but cannot be exceeded without producing unfavourable results. When this individual maximum dose has once been reached, it is repeated at certain intervals but not exceeded.

By making a series of dilutions in multiples of 2 , in such numbers that a dilution of 65,536 times is ultimately reached, and by means of the device of using only the upper half of the syringe in passing from one dilution to another, so that, for instance, the next dose following 
I c.c. of dilution $\frac{1}{12} \overline{8}$ is 0.55 c.c. of dilution $\frac{1}{64}$, Sahli has devised a very carefully graduated scheme for dosage, which certainly has advantage over the decimal system of dilution usually employed.

Bandelier and Roepke and the other members of the second school, while not failing to recognise that the observance of symptoms must also be supplemented by physical examination of the chest, and that focal reactions are more easily produced than general ones, yet fail to utilise this knowledge to the full advantage. Thus, after pointing out that "the view must be entirely put on one side that the curative process in tuberculin treatment takes place only with objective signs of reaction," and that "clinical observation teaches that a local effect on the focus of disease may make itself evident without a subjective feeling of illness or appreciable rise of temperature," Bandelier and Roepke state that "the common practice of observing the temperature curve only is quite sufficient for the purpose," $i$.e. of controlling of tuberculin inoculations. They hold that "the maxim to remain as close as possible to the reaction limit without well-marked or severe reactions occurring coincides with the experience that the most evident results have been obtained with slight reactions up to $38^{\circ} \mathrm{C}$. or a little over. The principle of the production of slight reactions will be more in place and easier to carry out in slighter and more limited cases of disease, in the slowly progressive forms associated with fibrosis, where nutrition and appetite are good, where weight is being put on and the temperature normal, and where susceptibility to tuberculin is slight. But in these cases the possibility of an overdose must still be borne in mind." And again, "It must be constantly kept in mind that the curative effect for the individual is dependent, not on the absolute, but on the relative size of the dose, i.e. that quantity of tuberculin is most advantageous which can at any time be just borne without (general) reaction. . . . Time must be allowed for the increase of dosage, and the same dose repeated, if necessary, several times if reaction occurs, with increase of the interval; or better, a return made to a smaller dose, increased again more slowly. The thought guiding action must be that any dose associated with fever is too high for the individual concerned, and that the smaller dose borne without reaction not merely suffices for therapeutic action but is actually of more value. Tolerance cannot be attained by violence but only by patiently persisting." The obvious criticism of all this is that while strongly upholding the advisability of a dosage which just falls short of a reaction in practice they fail to follow out their own precepts, and only determine the magnitude of their dosage by producing such reactions-at all events from time to time. Were more attention paid in the way $I$ have indicated to the resulting focal reactions, which they themselves admit are produced by 
dosages which fail to excite general reactions, guess-work and improper experimental determinations would be no longer necessary. In practice the determination of the dosage just short of that which will produce a general reaction is facilitated by the observation that a definite flattening of the temperature is coincident with the approach of the limit of tolerance. As regards the conclusion of treatment they write: "It must be our aim to attain to the highest possible doses of tuberculin, to reach the maximal dose. In slight cases a cure will be effected in this way. If the limit of apparently possible improvement is not then reached the maximal dose is to be repeated at increasing intervals as long as improvement continues, in order to retain the toxic immunity as long as may be, to stimulate the production of antibodies and to assist the healing processes. This injection of the absolute maximum we have ourselves prolonged for many months." Sahli well points out how utterly illogical is this artificial creation of a maximum dose at the containing capacity of the ordinary syringe, for this alone is the real reason why the maximum of dosage has been fixed at I c.c. The objection is, however, deprived of most of its sting in virtue of the fact that most members of this school stop considerably short of I c.c. as the maximum dose.

The procedures of the third school, that which ignores reactions and regards them as an incidental inevitable to a course of tuberculin, are based on the following considerations: (I) that the constitutional disturbances of the disease are due to lack of toxin tolerance on the part of the tissues; (2) that general reactions, like the constitutional disturbances, are unwelcome, and are obstacles to the attainment of the desired tolerance; (3) that therefore they are to be swept aside and overcome by brute force, as they have found can be done by the administration of massive doses, which must act by paralysing the mechanism of reactivity. They therefore waste no time, but begin with doses of 0002 c.c. of old tuberculin, increase the dosage, if necessary, till a general reaction is the result; when this occurs they repeat that dosage till the reaction begins to fail, when once more the dosage is increased. Some stop at a maximal dose of I c.c., others do not hesitate to go beyond this limit. In the case of some of this school the treatment is begun with P.T.O.; when a dosage of I c.c. has been attained with this, P.T. is substituted for it, treatment being concluded with a course of old tuberculin.

The objections to this method are several: (I) it is really only applicable to those whose general condition warrants such severe and oft-repeated constitutional disturbances; (2) that even in these the reactions may become more than the sufferers can bear, and treatment is abandoned; (3) that cases of mixed infection are considered to be 
unsuited to the treatment, and these comprise a very considerable proportion of the phthisical; (4) in advanced cases with pyrexia more harm than good may result.

There are other means of moving a heavy rock than by means of a cumbrous crane-a lever will do the same work with much less expenditure of energy and sometimes more expeditiously; similarly there are ways of raising tolerance than by the sledge-hammer-like blows of massive dosage. On the other hand, there is at least one class of case to which this method is the more applicable, namely, those cases of good general condition who, despite moderate degrees of pyrexia, have to go about their daily work, means of undergoing a careful course of immunisation in sanatorium or their own home being quite beyond them. Such cases are undergoing constant autoinoculation with varying doses of tuberculin at irregular intervals. The rest in bed which would control these being denied the patient, it becomes urgently necessary to overwhelm these irregular disturbances, which are unsuitable as stimuli for the establishment of immunity, under the greater and regulated waves set up by massive doses of tuberculin. That this objective is by no means impossible of attainment is certainly shown by the statistics of Wilkinson, Löwenstein and Götsch, and if this method be regarded as one to be employed in the lack of opportunity to the employment of a better, a definite sphere of usefulness can be assigned to it; it may even be regarded as the method of election for use on ambulatory patients, adapted to the needs and requirements, not of the classes, but of the masses.

\section{Treatment by the Induction of Auto-inoculations.}

Inman and Paterson have carefully investigated the relationship of the temperature curve to that of the opsonic content of the serum in pyrexial cases of phthisis. They found that the two curves roughly correspond but move in reverse directions. From this it may be inferred that rise of temperature corresponds to fall in content of the immune bodies generally of the blood, and that control of the temperature oscillations would result in the maintenance of the defensive mechanism at a steadier level. This has been confirmed by clinical observation; cases of irregular pyrexia wh ich are taking a downhill course improve markedly when the pyrexia is controlled by rest in bed. Sometimes ordinary rest will achieve this end ; sometimes absolute or "typhoid" rest is essential ; occasionally no degree of rest will control the irregular auto-inoculations. The thought suggested itself to Paterson whether it would not be better to utilise inoculations with the patient's own tuberculin produced by 
increasing the vascular supply to the infected foci by means of carefully ordered exercise than to introduce tuberculin from without. The very large percentage of the human race who do successfully autoinoculate themselves lends an $\grave{a}$ priori support to an affirmative answer. Paterson has therefore given an extensive trial to the treatment of cases of pulmonary tuberculosis by carefully graduated rest and exercise. Cases with pyrexia are sent to bed and kept at rest, ordinary or "typhoid," until their pyrexia is under complete control; they are then allowed up and given carefully graduated exercise. If the exercise results in a rise of temperature to over $99^{\circ} \mathrm{F}$. they are kept at rest until the temperature has steadied itself once more, when exercise is again permitted, and so on. In this way it is found that the organism can be accustomed to more and more exercise, until finally a hard day's manual labour may fail to elicit an immunising response as evidenced by rise of temperature beyond the patient's normal. The treatment has been found to be especially beneficial to cases which show little pyrexia and fail to improve beyond a certain point. Paterson reports excellent results from this form of treatment, but there are several objections to be taken to it: (I) It necessitates great individual attention and considerable care and judgment on the part of the physician in the assignment of the exercise to be taken by each patient. (2) It can only be carried out in a well-ordered sanatorium where the opportunity exists for furnishing the necessary tasks to the patients. (3) Auto-inoculations are induced the most easily when they are least desired, and are most difficult to induce when they are the most to be desired; i.e. as treatment advances, and arrest and cure are the more nearly reached, the organism gradually accustoms itself to stimuli, so that to keep up a high degree of immunity stimuli of increasing strength must needs be applied. On the other hand, as healing of the foci proceeds the difficulty of inducing hyperæmia in them increases, and the quantum of bacilli and their products which are carried into the circulation shows a corresponding decrease. It thus comes about that while it is easy to bring about marked relief of constitutional symptoms and rise in tolerance, it is rather more difficult to bring about arrest and much more difficult to bring about a cure.

It is much to be regretted that Paterson appears to have contented himself with an imperfect ideal, that he is content with having produced that degree of improvement which will enable the patient to return to his daily task, however severe this may be, without any resultant constitutional disturbance.

To the disappearance of all bacilli from the sputum, to the precise condition of the infected foci and consequently to the chance of permanence of the results obtained he appears to have paid too little 
heed. The procedure is based on sound reasoning and sound observation, and the introduction into it of suitable modifications, such as the addition of stimuli from without as those from within begin to fail, would tend to widen its applicability and increase its value. Whether auto-inoculatory procedures can be conducted with benefit in cases of mixed infection is very doubtful and must be left to the future to decide.

\section{Results.}

Upon this question I propose to say but little. Mere statistics are of little help in aiding the formation of a true estimate of the value of tuberculin in the treatment of pulmonary tuberculosis. The disease is often a very chronic one, and one of its worst features is its crippling effect on man's power of work and of production, and the consequent casting of his maintenance upon the shoulders of others. From the economic standpoint it is therefore even more important that recovery should be expedited in those who are destined in any case to recover, than that a certain additional percentage should be preserved to lead a life of semi-invalidism.

One of the most striking testimonies to the value of tuberculin treatment is the complete change of front in regard to its use which has once more come over the medical men throughout the world. Rivière and Morland point out, that whereas the percentage of sanatoria and public institutions in Germany which combined specific with hygienic treatment was only 29 in 1905 , it had risen to 57 in 1907 and to about 70 in I9Io. Many lung specialists who formerly were utterly opposed to it now use it extensively. Bandelier and Roepke cite the remarkable instance of Ritter, who, prejudiced against tuberculin, wished to collect evidence of its inefficacy. He therefore treated at his sanatorium a number of cases which had already undergone treatment without avail. The result is seen in the views he expressed in Ig08, that sanatorium physicians are not merely justified, but in a certain sense bound to make the widest possible use of tuberculin in the treatment of pulmonary tuberculosis.

Sahli says as regards himself: "Blind enthusiasm for tuberculin treatment can hardly be laid to my charge, but I honestly believe that it is the best weapon of modern times in the fight against tuberculosis."

The greater the experience of most specialists, not necessarily in the uses of tuberculin, but in the manifold aspects of the disease itself, the stronger as a rule is their advocacy of the remedy; the greater the experience of any specialist in tuberculin treatment itself the stronger as a rule is their advocacy, not that its use should be confined to 
specialists like themselves, but that it should be extended, and that all general practitioners should learn to avail themselves of its help. Thus Sahli, Bandelier and Roepke and Wilkinson each voice this sentiment, and Lenhart $z$ expressed much the same view when he said: "It is a defect in practice when tuberculin is not employed."

Rivière and Morland sum up the case for tuberculin succinctly and well in the following words: "But statistics apart, certain results may be said to be well established by clinical experience. The first and most striking of these is that phthisis treated with tuberculin before it has become open remains closed. The importance of this fact, in which there is practically unanimous opinion, can hardly be exaggerated. It is true that the same result has been claimed for hygienic treatment; it is also true that the vis medicatrix nature unfettered by art would have had the same result in a large proportion of cases; but there remains a proportion-it may be small-of closed pulmonary tuberculoses which will not get well, and with these tuberculin has been shown to be competent to deal. Early diagnosis-that is to say, really early diagnosis, before tubercle bacilli appear in the sputum-combined with specific treatment, insures completely against a breakdown. We believe that statistics have already shown the ability of tuberculin to increase the percentage of those who lose their sputum, or the tubercle bacilli contained in it, during hygienic treatment, and to extend the expectation of working efficiency after hygienic treatment ; but we are content to leave this to a more rigid demonstration. Of all these matters the tubercular patient is the final judge, and misled as he was by the disasters of $1890-9 \mathrm{I}$, there is no doubt that his experience of tuberculin under the new conditions is making him willing, and sometimes even anxious, to submit himself to treatment with the remedy."

It is with the earnest hope that the many hours of patient work which I have bestowed upon the various forms of bacterial invasion of the respiratory tract may not have failed to cast some light upon the origin, prophylaxis and treatment of the various distressing manifestations of infection and of pulmonary tuberculosis in particular, and thereby may have contributed in some small measure to the relief of human suffering, that I leave this record to the kindly consideration of the reader. 



\section{N DEX.}

Abscess of lung, 1 I9

Accessory sinuses, bacteriology of, 47 - - treatment of, 87

Albumen test, I 2

Allergie, 202

Antiformin method, 20

Antral disease, bacteriology of, 47

- - treatment of, 87

Asthma, bacteriology of, 52

- treatment of, 97

Auto-inoculation in phthisis, 230

Bacillus, Bordet-Gengou, 3 I

- coli communis, 40

- diphtheriae, 32

- Friedlander's, 38

- Hoffmann's, 32

- influenza, 31, 7t

- Koch-Weeks', 3I

- proteus, 39

- pyocyaneus, 39

- septus, 32

— tuberculosis, 20, 25, I92

- typhosus, 40

- xerosis, 32

Bacteriology of asthma, 52

- bronchitis, 52

- catarrh, nasál, 44

- post-nasal, 46

- common cold, 44

- laryngitis, $5 \mathrm{I}$

- ozena, I37

- phthisis, 55, 145

- preumonia, IO4

- rhinoscleroma, I39

- sinusitis, 47

- tracheitis, $5 \mathrm{I}$

- whooping-cough, 55, 122

Blister test, Woodcosk's, 204

Blood-agar, 23

Blood cultures, 106
Bronchitis, bacteriology of, 52

- treatment of, 90

Broncho-pneumonia, 1 I9

Calmette's test, 206

Capsule staining, 21

Catarrh, Eustachian, 50

- general treatment of, 82

- post-nasal, 46

- nasal, 44

- vaccine treatment of, 71,79

Cells of sputum, i3

Chemical examination of sputum, ir

Cultural methods, 23

Diphtheria bacillus, 32

- treatment of, 123

Dosage, control of, $65,80,9$ r, 156

Empyema, I I9

Flagella staining, 2 I

Focal reaction, 2 to

Friedlander's bacillus, $3 \mathrm{~S}, 73$

Fusiform bacillus, $+\mathrm{I}$

Gram's method of staining, 18

Hay-fever, I 34

Influenza bacillus, 31, 74

Inhalations, I 88

Intervals, control of, $6_{5}, 91$

Intra-dermal test, 203

Intra-tracheal injection, I 89

Koch-Weeks' bacillus, 3 I

Laryngitis, $5 \mathrm{I}, 83$

Leishman's stain, 13, 20

Lung abscess, I I9

- puncture ro 
Media, 23

Meningococcus, 35

Methods of collecting material, 8

- of staining, I 2, I 7

Micrococcus catarrhalis, 33, 72

- paratetragenus, 37,72

- tetragenus, 37

Mixed infection, import of, 141,147

- prevention of, 180

- - treatment of, 160,170

Morro's test, $2 \mathrm{O}_{+}$

Nasal catarrh, bacteriology of, 74

- - treatment of, 7I, 79

Ophthalmo-test, 206

Opsonic index in diagnosis, 2 I 4

Ozæna, bacteriology of, I 37

- treatment of, i 38

Percutaneous test, 204

Phthisis, bacteriology of, 55, 145

- mixed infections of, 55, I4 $\mathrm{I}$

- treatment of, I 40,192

Pneumococcus, 30,73

Pneumonia, bacteriology of, 104

- pathology of, I07

- prophylaxis of, 107

- treatment of, 103

Post-nasal catarrh, bacteriology of, 46

- - treatment of, 84

Preparation of vaccine, 75

Prophylaxis of catarrhs, $S_{4}$, ISo, I. 87

- of pneumonia, I07

Pyorrhoea alveolaris, bacteriology of, 4 I

- - importance of, 6I, I 25

- - treatment of, I 24

Reactions, 69, 80, 91, I 56

- table of, 69

- to tuberculin, 202

Reactivity, 202, 2 I I

Rhinoscleroma, bacteriology of, 139

- treatment of, I 39

\section{Salicylate test, I I}

Sero-albuminous contents of sputum, I 6

Sinusitis, bacteriology of, 47

- treatment of, 87

Spengler's staining methods, I9
Sputum, cells of, 13

- chemical examination of, I I

- method of collecting, 9

Staphylococcus, 27

Streptococcus, 28, 74

Streptothrix, 25

Subcutaneous test, 207

Swabs, method of taking, 8

Tonsillitis, bacteriology of, 4 I

- treatment of, 62

Tracheitis, $\mathbf{5} \mathbf{I}$

Tubercle bacillus, cultivation of, 25

- -- isolation of, 20,25

- - staining of, I8

Tuberculin, choice of, 2 I 9

- in diagnosis, 202

- in treatment, $22 \mathrm{I}$

- reaction, 207

Tuberculins, the, I99

Tuberculosis, defensive mechanism against, I 96

- mixed infection in, 56, 140, 160, 170, 180,

- treatment of, 140, 192

Unna's methylene-blue, I3

Vaccine, administration of, 77

- preparation of, 75

- treatment of asthma, 97

- - of bronchitis, 90

- - of catarrhs, 7 I, 79

- - of diphtheria, 123

- - of hay-fever, 134

- - of mixed infections, 160, 170

- - of ozæna, $1_{3} 8$

- - of phihisis, I 40,192

- - of pneumonia, IO3 $3_{3}$

- - of pyorrhøa alveolaris, I 24

- - of rhinoscleroma, I 39

- - of sinusitis, 87

- - of whooping-cough, I 2 I

-. - preliminaries to, 58

- - rationale of, 62

Von Pirquet test, 202

Whooping-cough, bacteriology of, 55, 122

- treatment of $12 \mathrm{I}$

Woodcock's blister test, 204

Ziehl-Neilsen's staining method, I 8 




$$
=
$$




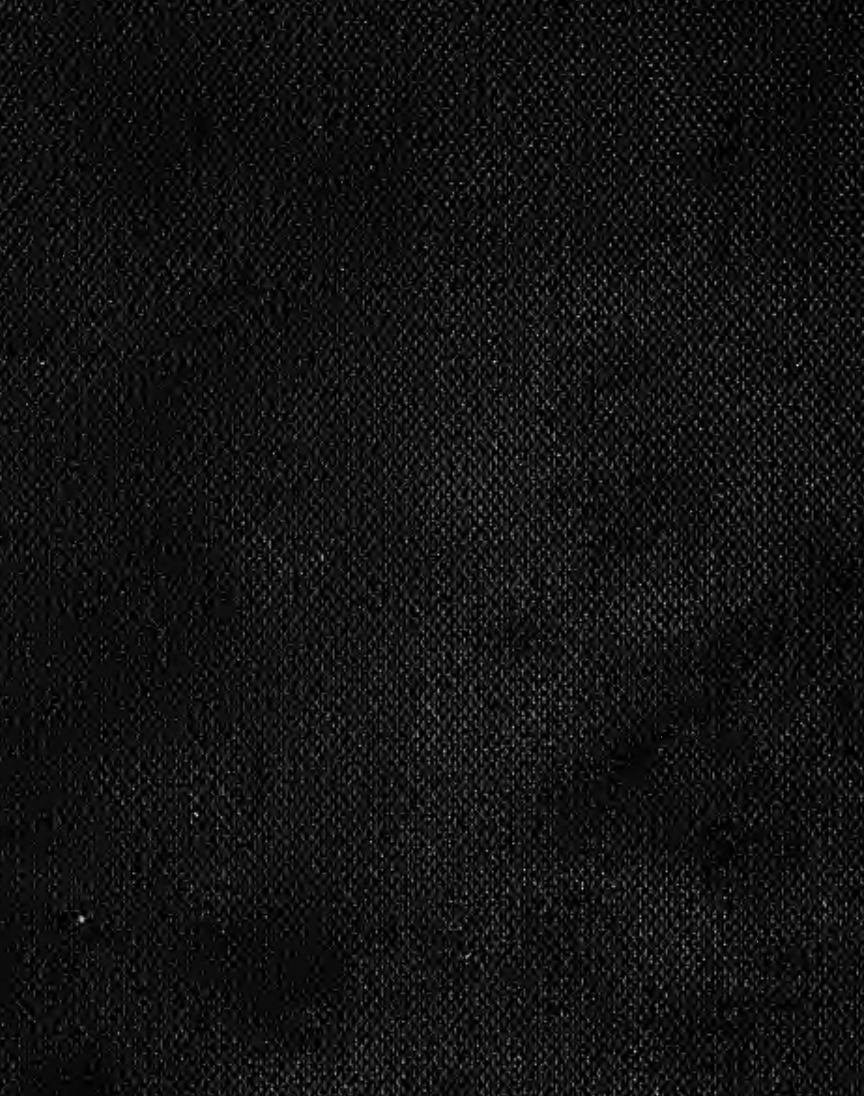

S.

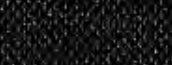

- 640

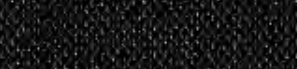

7.

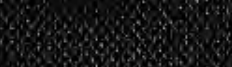

W

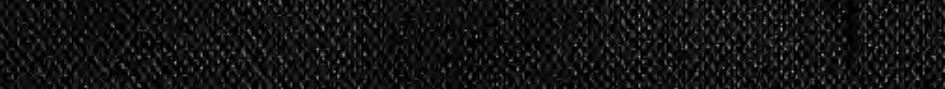

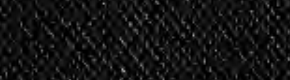

-

W

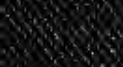

Now 